\title{
Multidimensional high harmonic spectroscopy
}

\author{
vorgelegt von \\ Ms. Phys. Valeriya Serbinenko \\ geb. in Kazan, Russland \\ von der Fakultät II - Mathematik und Naturwissenschaften \\ der Technischen Universität Berlin \\ zur Erlangung des akademischen Grades \\ Doktor der Naturwissenschaften \\ - Dr. rer. nat.- \\ genehmigte Dissertation
}

Promotionsausschuss:

Vorsitzender: Prof. Dr. Mario Dähne

Gutachter: Prof. Dr. Andreas Knorr

Gutachterin: Prof. Dr. Olga Smirnova

Gutachter: Dr. Vitali Averbukh

Tag der wissenschaftlichen Aussprache: 12. Juli 2017

Berlin 2017 


\section{Abstract}

Multidimensional high harmonic spectroscopy is a cutting-edge technique for studying the effect of the intense low-frequency laser fields on atoms and molecules. The high energy photons, emitted by a particle as a result of the interaction with the strong laser field, carry the information about the processes in atoms and molecules, which lead to the emission. Multidimensional character of the technique allows to extract parameters of the molecular and atomic ionization dynamics, that were inaccessible before in the same experiment, such as sub-cycle ionization rates, ionization and recombination times.

The Thesis presents in detail theoretical analysis of the multidimensional high harmonic generation process within semi-classical approach. Several generating field configurations are considered. The developed theoretical apparatus is then applied to describe multidimensional high harmonic spectroscopy experiments in helium. Initial analysis of the applicability of the technique for the study of multielectron dynamics in carbon dioxide is also performed.

The problems, solutions to which are presented in the Thesis, are:

- theoretical description of high harmonic generation in multicolor fields

- analysis of the gating techniques for the extraction of the parameters of electron trajectories in high harmonic generation in $\mathrm{He}$

- proposition of an alternative reconstruction procedure of electron trajectory parameters, that allows one to access to all of them simultaneously

The main results of our work are:

- Based on theoretical analysis of multidimensional high harmonic generation, we showed, that optimization of the two-dimensional harmonic 
signal is achieved at each step of the process: ionization, propagation, dynamics in the ion and recombination

- We demonstrated, that multidimensional high harmonic spectroscopy is a very sensitive tool allowing one to extract parameters of electron trajectories, such as complex ionization time and complex electron momentum, from the observed harmonic signal

- We developed and applied theory to the analysis of two-color high harmonic generation experiments of He atom

- We performed initial analysis of two-color HHG experiments in carbon dioxide, which was necessary for further investigation and reconstruction of ionization dynamics 


\section{Acknowledgements}

I am grateful to my supervisor Dr. Olga Smirnova for her support during PhD. Also, I thank Olga for giving me a lot of freedom in my research activity, plenty of room for the creativity and encouraging the broadening of my horizons with numerous conferences, seminars and work trips to our collaborators.

I thank Lisa Torlina for the friendship and going through the PhD together. I thank Misha Ivanov for helpful insights, discussions and ideas. I thank Felipe Morales for his sound advice about coding and for the exceptional and very effective help with any IT problems I had. I'm very grateful to all members of the theory group, especially, Alex Harvey, Maria Richter, Felipe Morales, Foudhil Bouakline and Danilo Brambila for many interesting and constructive discussions.

I am very grateful to our experimental collaborators, the group of Nirit Dudovich from Weizmann Institute of Science, for the warm welcome and amazing time I spent in their lab. I thank Nirit for remarkable and beautiful experiments her group performed. I thank Nirit Dudovich, Oren Pedatzur, Hadas Soifer, Barry Bruner, Ayelet Uzan, Gil Porat and Gil Orenstein for many discussions, exchange of ideas, their great experiments and the fun, I had in their group.

I thank Bettina Becker, the most helpful person in the institute or in the whole world, who made the bureaucratic problems very easy. There was no question she didn't answer and no problem she didn't solve quickly and efficiently.

Last, but not least, I'm very grateful to Sebastian Eilzer, Saida Asbai, Robert Gerlach, Julia Polgar and Enrique Medarde for their amazing support and help. 


\section{Publications arising from work}

B. D. Bruner, Z. Mašìn, M. Negro, F. Morales, D. Brambila, M. Devetta, D. Faccialá, A. Harvey, M. Ivanov, Y. Mairesse, S. Patchkovskii, V. Serbinenko, H. Soifer, S. Stagira, C. Vozzi, N. Dudovich and O. Smirnova,

Multidimensional high harmonic spectroscopy of polyatomic molecules: detecting sub-cycle laser-driven hole dynamics upon ionization in strong mid-IR laser fields

Faraday Discussions, 194, 369 (2016)

O. Pedatzur, G. Orenstein, V. Serbinenko, H. Soifer, B. D. Bruner, A. J. Uzan, D. S. Brambila, A. G. Harvey, L. Torlina, F. Morales, O. Smirnova and N. Dudovich,

Attosecond tunnelling interferometry

Nature Physics 11, 815 (2015)

B. D Bruner, H, Soifer, D. Shafir, V. Serbinenko, O. Smirnova and N. Dudovich,

Multidimensional high harmonic spectroscopy

Journal of physics B: Special Issue on Coherence and Control in the Quantum World 48, 17 (2015)

V. Serbinenko and O. Smirnova,

Multidimensional high harmonic spectroscopy: a semi-classical perspective on measuring multielectron rearrangement upon ionization Journal of physics B 46, 171001 (2013)

H. Soifer, M. Dagan, D. Shafir, B. D. Bruner, M. Yu. Ivanov, V. Serbinenko, I. Barth, O. Smirnova and N. Dudovich, 
Spatio-spectral analysis of ionization times in high-harmonic generation

Chemical Physics 414, 176 (2013)

F. Morales, I. Barth, V. Serbinenko, S. Patchkovskii and O. Smirnova,

Shaping polarization of attosecond pulses via laser control of electron and hole dynamics

Journal of Modern Optics 59(15), 1303 (2012) 


\section{List of abbreviations}

$\begin{array}{ll}\text { ADK } & \text { Ammosov, Delone and Krainov } \\ \text { ATI } & \text { Above Threshold Ionisation } \\ \text { CEP } & \text { Carrier-Envelope Phase } \\ \text { FFT } & \text { Fast Fourier Transform } \\ \text { FWHM } & \text { Full Width at Half Maximum } \\ \text { HHG } & \text { High Harmonic Generation } \\ \text { HOMO } & \text { Highest occupied molecular orbital } \\ \text { IR } & \text { Infra-Red } \\ \text { KFR } & \text { Keldysh-Faisal-Reiss } \\ \text { MHHS } & \text { Multidimensional High Harmonic Spectroscopy } \\ \text { NSDI } & \text { Non-Sequential Double Ionisation } \\ \text { SAE } & \text { Single active electron } \\ \text { SFA } & \text { Strong Field Approximation } \\ \text { SH } & \text { Second Harmonic } \\ \text { TDSE } & \text { Time Dependent Schrödinger Equation } \\ \text { UV } & \text { Ultra-Violet } \\ \text { XUV } & \text { eXtreme Ultra-Violet }\end{array}$




\section{Contents}

$\begin{array}{ll}\text { Publications arising from work } & 7\end{array}$

$\begin{array}{lr}\text { List of abbreviations } & 9\end{array}$

1 Introduction 13

1.1 Early history of High Harmonic Generation . . . . . . . . . . . . 14

1.2 Classical theory of High Harmonic Generation . . . . . . . . . . 15

1.2.1 Single active electron approximation . . . . . . . 16

1.2.2 Ionization step in the classical theory of strong fieldatom interaction . . . . . . . . . . . 18

1.2.3 Propagation step . . . . . . . . . . . . . . . . . . . 23

1.2.4 Recombination step . . . . . . . . . . . . 25

2 Application of High Harmonic Generation to studies of atomic and $\begin{array}{ll}\text { molecular structure and dynamics. } & 31\end{array}$

2.1 Structural information . . . . . . . . . . . . . . 32

2.1.1 High harmonic Generation in atoms . . . . . . . . . . 32

2.1.2 Molecular alignment . . . . . . . . . . . . . 33

2.1.3 High harmonic generation in aligned molecules: General features . . . . . . . . . . . . . . 37

2.1.4 High harmonic generation in aligned molecules: Structural features of Molecular Orbitals . . . . . . . . . 40

2.1.5 Molecular tomography with high harmonic generation . . 42

2.2 Nuclear and electronic dynamics resolved via High Harmonic

Generation . . . . . . . . . . . . . . . 44

2.2.1 Nuclear dynamics via HHG . . . . . . . . . . . . . 44

2.2.2 High harmonic generation: multielectron effects . . . . 46 
2.3 Multicolor schemes for high harmonic spectroscopy . . . . . . . 48

3 Theory of High Harmonic generation in multicolor fields 55

3.1 Strong Field Approximation . . . . . . . . . . . . . 57

3.2 Saddle point approximation . . . . . . . . . . . . . 60

3.3 Analysis of the saddle point equations: complex (quantum) trajectories. . . . . . . . . . . . . . . . . 64

3.3.1 Harmonic dipole in the frequency domain . . . . . . . . . 67

3.3.2 Harmonic dipole in the time domain . . . . . . . . . . . 69

3.3.3 Including multielectron dynamics to the harmonic dipole 70

4 Analysis of multicolor saddle points

4.1 Saddle point approximation in the two-color perpendicular scheme of $\mathrm{HHG} \ldots \ldots \ldots \ldots$. . . . . . . . . . . . . . . . 75

4.1.1 Second harmonic field as a perturbation . . . . . . . 75

4.1 .2 Strong second harmonic field . . . . . . . . . . . . . 88

4.2 Saddle points for the parallel configuration of the two-color field 95

4.3 Saddle points for the multicolor fields . . . . . . . . . . . . . 103

5 Gating with two-color fields for the reconstruction of the parameters of electron trajectories.

5.1 Reconstruction of time of exit from the tunnelling barrier . . . . 115

5.2 Reconstruction of the imaginary part of the ionization time . . . 118

5.2.1 Attosecond tunnelling interferometry . . . . . . . . . . 118

5.2 .2 Multicolor scheme . . . . . . . . . . . . . . . . 122

6 Outlook: dynamics in $\mathrm{CO}_{2} \quad 135$

$\begin{array}{llr}7 \text { Conclusion } & 147\end{array}$

$\begin{array}{ll}\text { Bibliography } & 153\end{array}$ 


\section{Introduction}

Fast advances in technology are often initiated by the desire for, and the continual growth of, our understanding of the natural world. Development of modern tools in all areas of scientific research is both necessary and natural part, which goes hand-in-hand with accommodating our new knowledge about nature. For example, thorough understanding of chemical reactions opens the possibilities for their precise control, with applications as diverse as creation of new materials and medicine, cheaper production of existing chemical compounds, more efficient or new recycling methods, higher performances of batteries, renewable and non renewable energy plants, etc.

Tremendous joint efforts by researches in physics and chemistry are taken in order to visualize chemical reactions with femtosecond to sub-femtosecond temporal and angstrom-scale spatial resolution, to observe the dynamics of nuclei and electrons during the formation of new chemical bonds. While moving of the nuclei happens on a femtosecond ( $\left.1 \mathrm{fsec}=10^{-15} \mathrm{sec}\right)$ timescale, multielectron rearrangements in atoms and molecules, leading to the breaking and formation of the chemical bonds, can occur as fast as within hundreds of attoseconds ( 1 asec $=10^{-18} \mathrm{sec}$ ). Rapidly evolving field of attosecond physics explores these ultrafast dynamics, striving to realize the long-standing dream of visualizing ultrafast molecular dynamics and molecular structures in individual molecules.

With this goal in mind, a number of new tools have been developed in ultrafast optics recently, including but not limited to: attosecond streaking spectroscopy [1-5], photo-electron spectroscopy of correlated (non-sequential) double ionization (NSDI) [6-9], laser-induced electron diffraction [10-13] and time-resolved electron holography [14] using above-threshold ionization (ATI) [15, 16], and high harmonic generation (HHG) [17, 18]. 
High harmonic generation is one of the most rapidly developing and widely discussed topics in the field of ultrafast optics, with many applications. Numerous successful experiments use HHG as a source of coherent, bright, short pulses of XUV radiation. Indeed, the basic process of HHG is the fundamental technology that underlies modern attosecond light sources. Our discussion, however, will focus on a very different yet equally exciting application of this phenomenon, namely, the application of high harmonic generation as a highly nonlinear spectroscopy for studying molecular structures and dynamics.

\subsection{Early history of High Harmonic Generation}

High harmonic radiation is a result of a highly nonlinear interaction of an intense laser pulse with matter. The typical high harmonic spectrum in a centrally symmetric medium is characterized by a series of peaks at odd harmonics of the pump laser beam and consists of a rapid fall-of in the area of low harmonics followed by an extended plateau and an exponentially rapid cut-off, after witch no harmonics are observed.

For the first time, high order harmonic generation in rare gases was observed in experimental works [17] and [18]. High order odd harmonics were generated by using intense laser pulses of ultraviolet light at $248 \mathrm{~nm}$ in [17] and infra-red light at $1064 \mathrm{~nm}$ in [18]. Plateau and cut-off was observed in both experiments in several rare gases such as Ne, Ar, Kr and Xe.

After these pioneering experiments, numerous theoretical and experimental works have followed. Examples of some of the experimental studies include works on the cut-off extension to higher frequencies [19-23]; optimization of the parameters of the generating media [24-32] and of laser parameters [33-35]; control of phase matching conditions [36-40].

Theoretical work on describing high harmonic generation spectra included numerical simulations of the time-dependent Schrödinger equation in [41-44], numerical calculations using the Floquet states [45], dressed states [46] and classical trajectories [47]. Extremely important was the work [48], where the empirical law for the cut-off energy of the harmonic spectra, and its universal 
character, was discovered. This empirical law was explained with the classical theory of high harmonic generation introduced in [49-51]. In [49], the semiclassical three-step model of HHG was fully built. This model unified several important strong-field phenomena: HHG, highly efficient double ionization of atoms, and the generation of high energy electrons during ionization in infrared laser fields. Very soon, the first formulations of the quantum-mechanical theory followed in $[52,53]$, using single active electron approximation and the so-called strong field approximation. These theoretical works will be presented later in the current manuscript.

This thesis is organized as follows: first, we present the classical three-step model of high harmonic generation. It offers very good qualitative and intuitive understanding of this phenomenon. Next, in chapter 2 we review the advances in the application of HHG to investigating molecular structure and electronic dynamics. In chapter 3 we present semi-classical theory of high harmonic generation in multicolor fields. Analysis of saddle points in a few configurations of multicolor field is presented in chapter 4. The results, obtained from the analysis of saddle points, are implemented to the description of the gating techniques with multicolor fields in the chapter 5 . In chapter 6 we present an outlook on the implementation of multidimensional high harmonic generation to study molecular dynamics in $\mathrm{CO}_{2}$ in two-color fields. For the analysis we use theory, developed in previous chapters. In the last section of the thesis we conclude and summarize our findings.

\subsection{Classical theory of High Harmonic Generation}

The classical model of high harmonic generation during the interaction between an intense, low-frequency laser field and a single atom or molecule was formulated in [49-51]. In literature, this model is also referred to as the "three-step model" or the "simple man's model", which we will also use in our narrative. In this section we will consider the case of a linearly polarized laser field only.

The classical model describes the generation of high harmonics of the incident laser light as a sequence of three steps: ionization of an atom and the 'birth' 
of a 'free' electron, motion of this electron in the continuum, and finally the radiative recombination of this electron with the parent ionic core. The radiative recombination step takes the system 'electron+parent ion' back to its initial state and converts the energy accumulated by the continuum electron during its motion in the laser field into a high energy photon. Let us consider these three steps within the classical approach in detail, focusing also on the key approximations made in this model. Some of these approximations are also used by its quantum counterpart.

\subsubsection{Single active electron approximation}

The first assumption made in the classical model is that of a single active electron responding to the intense laser field and moving in the self-consistent field of other electrons.

Complete description of the interaction of atoms and molecules with the laser field is given by an exact solution of the time dependent Schrödinger equation (TDSE):

$$
i \hbar \frac{\partial}{\partial t} \Psi(t)=\hat{\mathcal{H}}(t) \Psi(t)
$$

where $\Psi(t)$ is a complete multielectron wave function. Hamiltonian $\hat{\mathcal{H}}$ contains stationary molecular or atomic Hamiltonian and time dependent potential $V_{L}(t)$, that describes the interaction of atom or molecule with the laser field:

$$
\hat{\mathcal{H}}=\hat{H}_{0}+\hat{V}_{L}(t)
$$

Exact solution of the dynamic multielectron problem, especially in the presence of an intense (non-perturbative) laser field, is not possible. Approximations have to be made. Importantly, in the case of intense laser-matter interaction, we cannot use standard perturbative approach, treating laser field as a weak perturbation, because the forces acting on the electron cloud from the laser field are comparable with the intra-atomic Coulomb forces. 
The standard assumption about the exact time-dependent $N$-electron wave function $\Psi(t)$ is to use the Hartree-Fock method, where $\Psi(t)$ is substituted by a product of single-electron time-dependent wave functions $\psi_{i}(t)$ :

$$
\Psi(t)=\hat{A} \psi_{1}(t) \psi_{2}(t) \ldots \psi_{i}(t) \ldots \psi_{N}(t)
$$

and $\hat{A}$ anti-symmetrizes the wavefunction. Next, all electrons, except for one valence electron, are assumed to be frozen. Together with the nucleus they create single-electron effective potential. The one remaining 'active' electron is allowed to interact with the intense laser field in the presence of the effective core potential. The effective potentials are constructed for each atom or molecule according to reproduce as well as possible the structure of its electronic orbitals. As long as double ionization remains much less likely then single ionization, only one active electron is considered, and multi-electron excitations by the low-frequency laser field are neglected. This assumption is called single active electron (SAE) approximation $[51,54,55]$.

The SAE approximation has been very successful in describing the interaction of many atoms and small molecules with infrared laser fields, because twoelectron excitations in these systems typically lie very far in energy, a lot higher than single excitations, and thus can be neglected. This condition is indeed very well met by noble gases and small molecules such as nitrogen, oxygen, carbon dioxide. There, the SAE approximation gives very good results. In other systems, e.g. large molecules or already in alkaline-earth metal atoms, SAE approximation fails or needs corrections [56-59]. In particular, in alkalineearth metals, double excitations lie below the first ionization threshold, thus SAE approximation can become inaccurate.

Finally, the intense laser field is described classically:

$$
V_{L}(t)=-e f(t) F \cos \left(\omega_{L} t+\phi\right)
$$

where $e$ - is the electron charge, $F$ - field strength of the electrical field of the laser wave, $f(t)$ - the envelope of the laser pulse, $\omega_{L}$ - frequency of the laser wave, $\phi$ - initial phase of the laser wave. 
Thus, in SAE approximation time-dependent Schrödinger equation is:

$$
i \hbar \frac{\partial}{\partial t} \psi_{i}(t)=\hat{H}(t) \psi_{i}(t)
$$

where $\psi_{i}(t)$ is the wave function of the interacting electron. Hamiltonian $\hat{H}=$ $\hat{H}_{0}+V_{\text {eff }}(t)$ contains field free Hamiltonian $\hat{H}_{0}$ and effective single-electron potential $V_{\text {eff }}(t)$, that describes the interaction of one electron with the strong laser field and the frozen core. For the specific atoms and molecules $\hat{H}_{0}$ and $V_{\text {eff }}(t)$ are constructed accordingly to their electronic structures and described conditions with the possible level of approximations.

\subsection{2 lonization step in the classical theory of strong field-atom interaction}

The first prominent step in the process of interaction of a strong low-frequency laser field with atoms or molecules is ionization. In low-frequency (typically, infra-red) laser fields, ionization is described as tunneling, first formulated in [60] and later in [61-63]. In strong low-frequency fields, the tunnelling model of ionization is a very good approximation, which was confirmed by numerous theoretical and experiential studies (see e.g. reviews [64-68]). 'Low-frequency' means that the electron dynamics inside the potential well adiabatically follows the change of the field. The term 'intense' implies that the laser field strength is sufficient to bend the potential barrier for the tunneling ionization to become significant. In the language of photons, ionization is multi-photon, meaning that the system absorbs sufficient number of photons to overcome the binding energy $I_{p}$.

Schematically, the tunneling ionization, and all the three steps of HHG, are shown in Figure 1.1. The binding potential well of the atom or molecule is deformed every half cycle of the strong linearly polarized laser field $E=E_{0} \cos \omega t$ to the extent that the probability of tunneling through the created potential barrier is non-vanishing. Tunneling is repeated every laser half-cycle and results in the series of bursts of the electron wave packets near peaks of the laser field. A very important parameter for the strong field ionization theories, such 


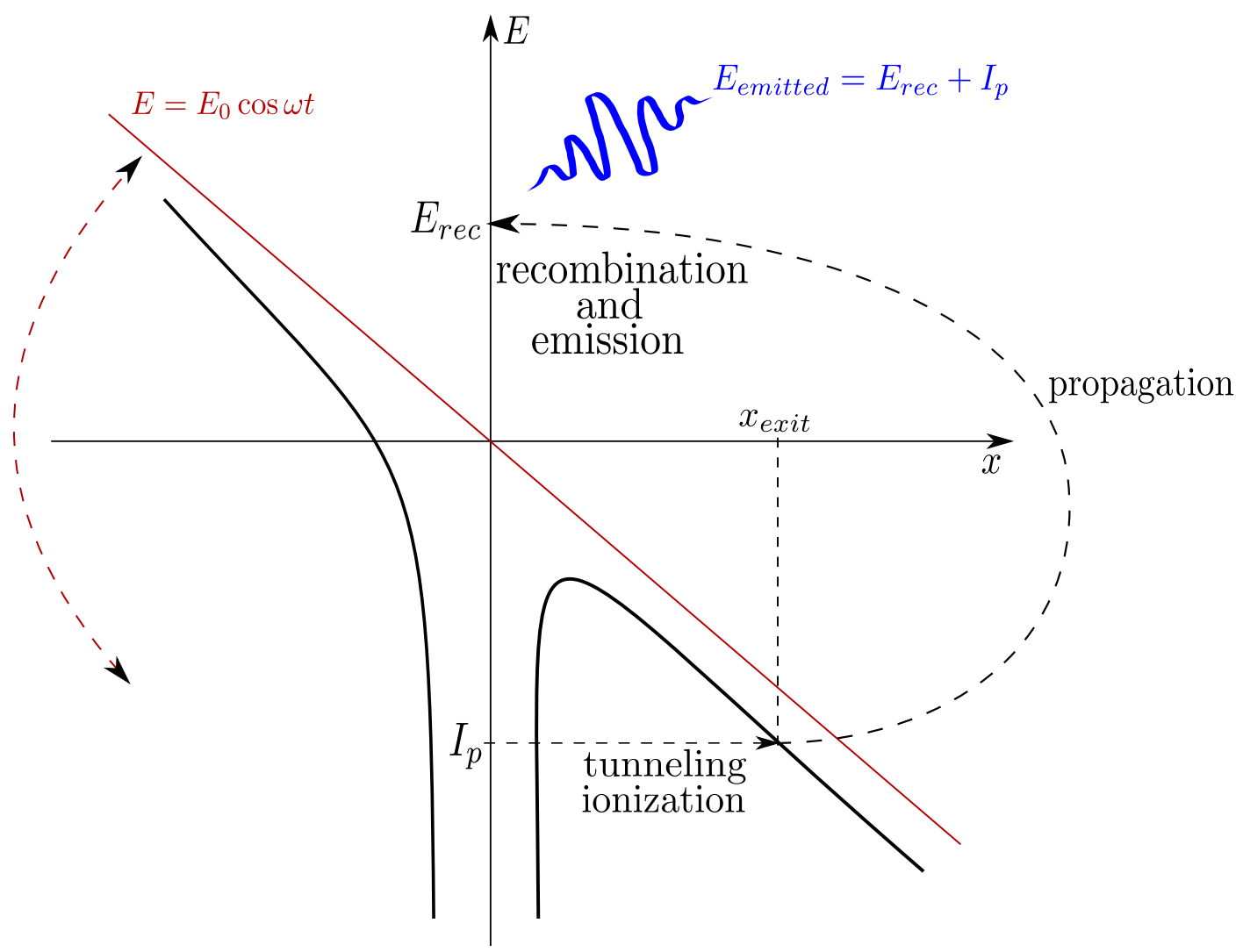

Figure 1.1: The schematic representation of a 3-step model of HHG [49]

as the Keldysh-Faisal-Reiss (KFR) theory [60, 69, 70], the Perelomov-PopovTerent'ev (PPT) theory [61-63] and the Ammosov-Delone-Krainov (ADK) theory [71], is the so-called Keldysh parameter [60]:

$$
\gamma=\sqrt{\frac{I_{p}}{2 U_{p}}}
$$

where $I_{p}$ is the ionization potential and $U_{p}=F^{2} / 4 \omega_{L}^{2}$ is the ponderomotive energy, i.e. the average energy of the electron oscillating in the electromagnetic field of frequency $\omega_{L}$ and field amplitude $F$. The Keldysh parameter shows whether it is fair to assume, that the electron tunnels through a static barrier (adiabatic tunnelling), or that the barrier changes during the tunnelling process (non-adiabatic tunnelling) [72].

Adiabatic tunnelling occurs when the value of the Keldysh parameter is small, 
$\gamma \ll 1$. It is easy to see, if $\gamma$ is expressed as follows:

$$
\gamma=\sqrt{\frac{I_{p}}{2 U_{p}}}=\frac{\omega_{L} \sqrt{2 I_{p}}}{F}=\omega_{L} \tau
$$

where $\tau=\sqrt{2 I_{p}} / F$ is so-called tunnelling time, the time needed for a classical particle to travel through the potential barrier, if the motion was classically allowed, as discussed in [60]. The relation $\omega_{L} \ll 1 / \tau$ now shows that, indeed, we can assume tunnelling of the electron through a (nearly) static barrier.

When the values of the Keldysh parameter are close to unity or higher $(\gamma \sim 1$ or $\gamma \gg 1$ ), the tunnelling barrier is no longer static, and the laser-induced changes in the shape of the potential well and the shape of the potential barrier during the ionization step can no longer be ignored. The electron energy, which remains constant in the static tunnelling limit (the Hamiltonian is timeindependent), can now change during the ionization step. As $\gamma$ increases, the frequency domain picture of multiphoton absorption becomes more appropriate.

The probability of ionization in linearly polarized laser field from an energy level with orbital angular momentum $l$ and its projection $m$ on the direction of the field, in the regime $\gamma<1$ (adiabatic approximation) is [61-63]:

$a_{l m}=I_{p}|C|^{2}\left(\frac{6}{\pi}\right)^{1 / 2} \frac{(2 l+1)(l+|m|) !}{2^{|m|}|m| !(l-|m|) !}\left(\frac{F}{2 F_{0}}\right)^{|m|+\frac{3}{2}} \exp \left\{-\frac{2 F_{0}}{3 F}\left(1-\frac{1}{10} \gamma^{2}\right)\right\}(1.8)$

where $F_{0}=\left(2 I_{p}\right)^{2 / 3}$ is often referred to as the effective intra-atomic field strength. $C$ is a dimensionless constant associated with the asymptotic behaviour of the radial wave-function in the region where the intra-atomic potential can already be described as a purely Coulomb potential, $\propto C \exp ^{-\kappa r} / r(\kappa r)^{1 / \kappa}$ (see details in [61]), $\kappa=\sqrt{2 I_{p}}$.

Let us consider the properties of the ionization probability Eq. (1.8). We examine the simplest case of ionization from the ground state of the hydrogen atom. In this case the value of the dimensionless constant is known exactly, $C=2$ [61], the ionization potential $I_{p}=13.5984 \mathrm{eV}\left(I_{p}=0.5\right.$ a.u. $)$ and $l=0$, $m=0$ for the $1 s$ electron in hydrogen. Let us consider the parameters of the 
laser field that have been used for many experiments in high harmonic generation: $800 \mathrm{~nm}$ laser light (corresponds to the angular frequency in atomic units $\omega=0.057$ a.u.) and intensity of the laser field $I=10^{14} \mathrm{~W} / \mathrm{cm}^{2}$ (corresponds to the field strength amplitude in atomic units $F=0.053$ a.u.). We are now going to change a few parameters, one by one, keeping other parameters fixed, to illustrate the behaviour of the ionization probability.

In figure 1.2 we present the dependences of the ionization probability and the Keldysh parameter on the field strength amplitude $F(a)$, ionization potential $I_{p}(b)$, the laser frequency $\omega_{L}(c)$ and the tunneling time $\tau(d)$. The dependence of the ionization probability on the field strength $(a)$ is easy to understand: the stronger the field, the thinner is the barrier, the easier it is for the electron to tunnel out. Similar situation is with the increase of the laser frequency $(c)$ : fewer photons with higher energies are needed from the field for the ionization to happen, making ionization easier.

When we change the ionization potential $I_{p}$ on the second graph, naturally, we no longer look exactly at the ground state of the hydrogen atom, but the graph (b) illustrates simple and important principle: with the increase of the ionization potential of the atom or molecule, it becomes exponentially harder for the electron to tunnel.

The change of the tunnelling time $\tau$ in panel $(d)$ is included in the formula Eq. (1.8) through the Keldysh parameter $\gamma=\omega_{L} \tau$. Ionization potential and intra-atomic field strength were modified accordingly, changing the ground state of hydrogen atom to the artificial construction as in the previous case. The larger the tunnelling time is, the higher is the ionization potential for the constant field strength, and the electron has to tunnel through a thicker barrier. The ionization probability decreases exponentially, as shown in Figure 1.2d. The tunnelling time shows the relation between ionization potential and the field strength and is linked to the thickness of the barrier $d_{\text {barrier }}=\tau v$, where $v$ is the average velocity under the barrier. Thus, its physical meaning is directly connected with the ionization probability and should be regarded as a parameter used to describe ionization without calling for the formula of the ionization probability every time: large or small tunnelling time shows small or large ionization probability. 
Ionization probability

Keldysh parameter
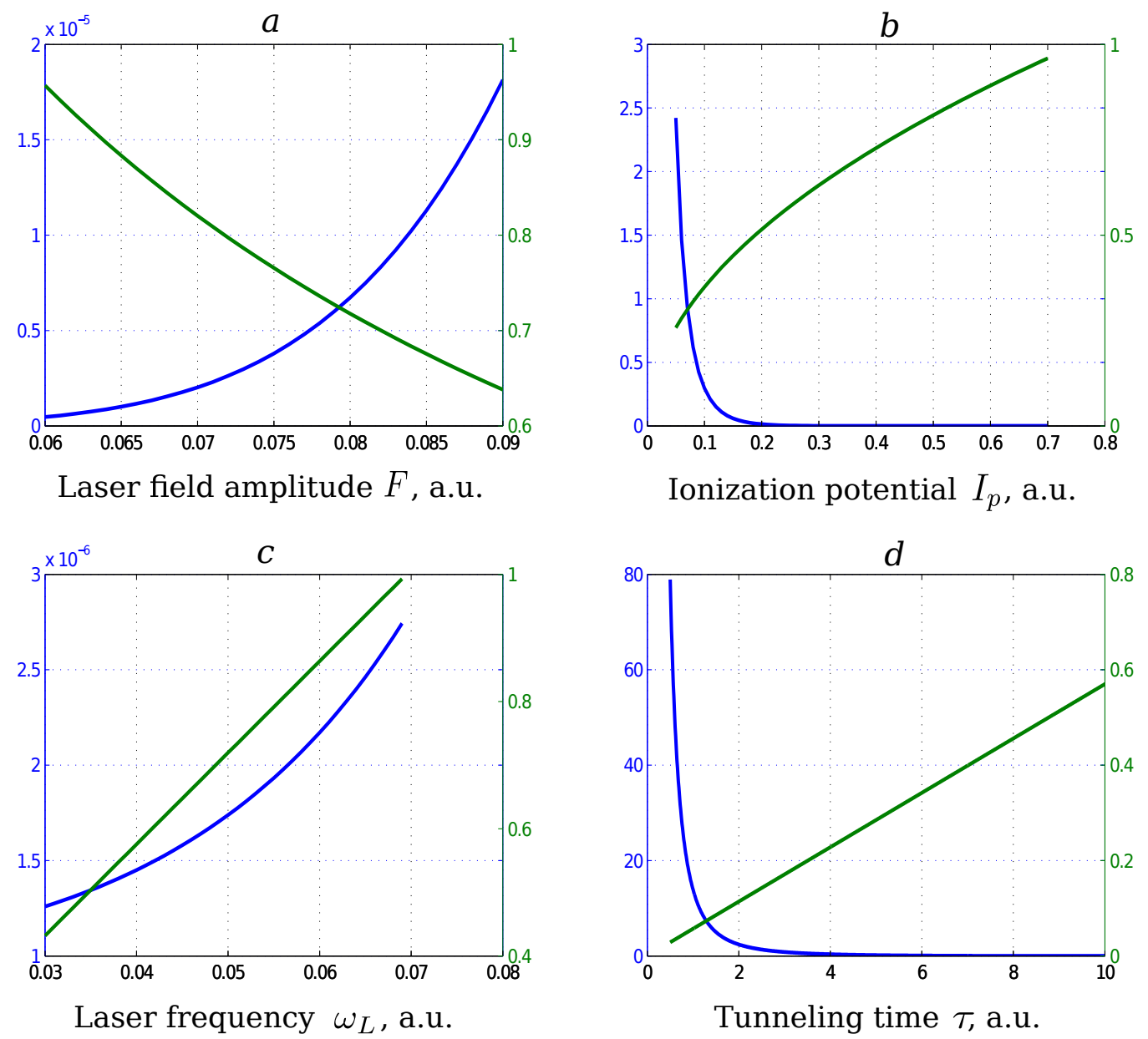

Figure 1.2: Probability of ionization and the Keldysh parameter, according to the PPT theory [61]. The graphs show the dependence of the ionization probability $a_{l m}$ (blue) and the Keldysh parameter $\gamma$ (green) on various parameters: a) the laser field amplitude $F$, b) the ionization potential $I_{p}, c$ ) the laser frequency $\omega_{L}$ and $d$ ) the tunneling time $\tau$. Parameters of calculation: ionization potential $I_{p}=13.5984 \mathrm{eV}$, laser wavelength $\lambda=800 \mathrm{~nm}$, intensity of the laser field $I=10^{14} \mathrm{~W} / \mathrm{cm}^{2}$. 


\subsubsection{Propagation step}

Starting with the pioneering work of P. Corkum [49], the propagation of the electron after ionization is often described by the classical Newton equations:

$$
\ddot{x}=-F_{L}(t)
$$

where the laser field can include an envelope, $F_{L}(t)=f(t) F \cos \left(\omega_{L} t+\phi\right)$. The initial conditions assume that just after tunneling the electron has zero velocity $v\left(t=t_{i}\right)=0$. In low-frequency laser fields, where the electron oscillation amplitude, $F / \omega_{L}^{2}$, is much larger than the width of the tunneling barrier, the initial displacement of the electron from the origin is also often neglected, setting the coordinate at the ionization moment $t_{i}$ to zero, $x\left(t=t_{i}\right)=0$, where $t_{i}$ is the moment of ionization.

If the envelope $f(t)$ changes slowly with time, then equations for the velocity and coordinate of the electron are:

$$
\begin{array}{r}
v(t)=-f(t) \frac{F}{\omega_{L}} \sin \left(\omega_{L} t+\phi\right)+v_{0}, \\
x(t)=f(t) \frac{F}{\omega_{L}^{2}} \cos \left(\omega_{L} t+\phi\right)+v_{0} t+x_{0}
\end{array}
$$

The initial conditions yield:

$$
\begin{array}{r}
v_{0}=f\left(t_{i}\right) \frac{F}{\omega_{L}} \sin \left(\omega_{L} t_{i}+\phi\right), \\
x_{0}=-f\left(t_{i}\right) \frac{F}{\omega_{L}^{2}} \cos \left(\omega_{L} t_{i}+\phi\right)-f\left(t_{i}\right) \frac{F t_{i}}{\omega_{L}} \sin \left(\omega_{L} t_{i}+\phi\right)
\end{array}
$$

Let us consider the properties of the classical electron trajectories 1.10, 1.11. Depending on the time of ionization, there are few possibilities for the electron behaviour. In figure 1.3a, we see a few electron trajectories that started at different times of the laser cycle $F(t)=F \cos \omega t$ within the first half period of the oscillation. The trajectories $x_{i}(t)$ may make multiple returns to the position of the parent ion ( 0 in the figure), or they may return once, or the electron may also leave the vicinity of the ion for good without ever returning back. 


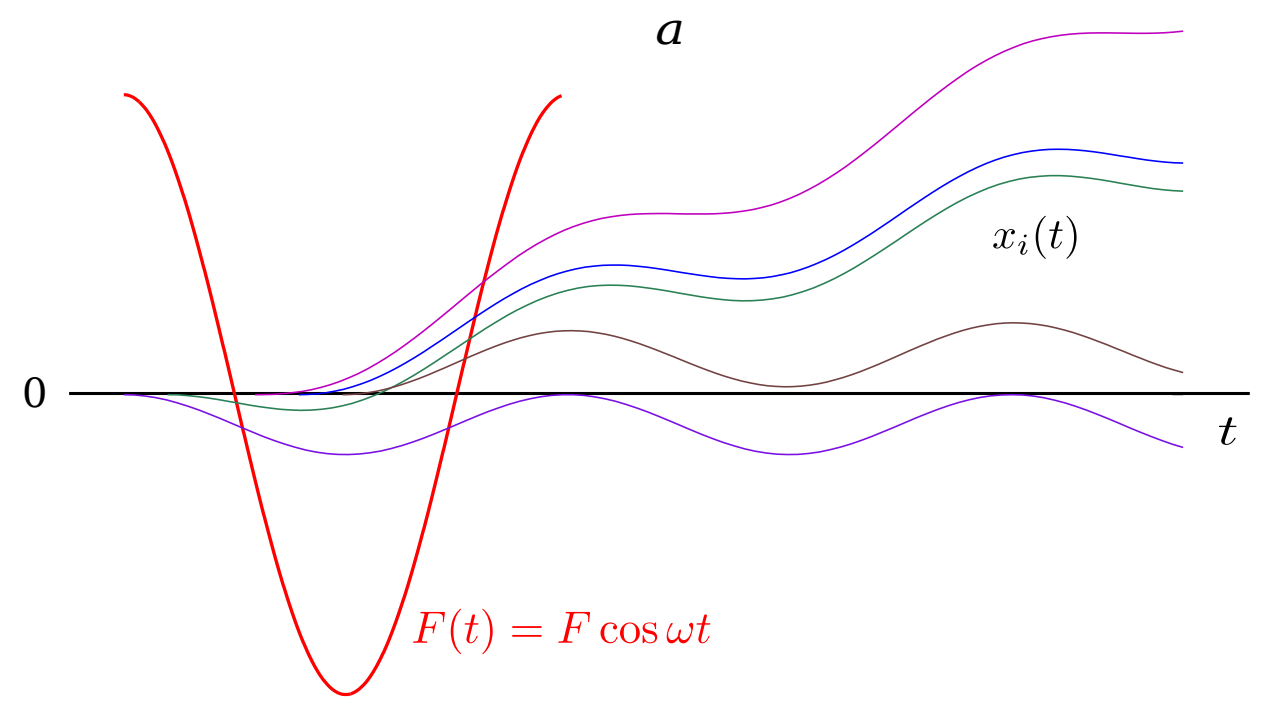

$b$

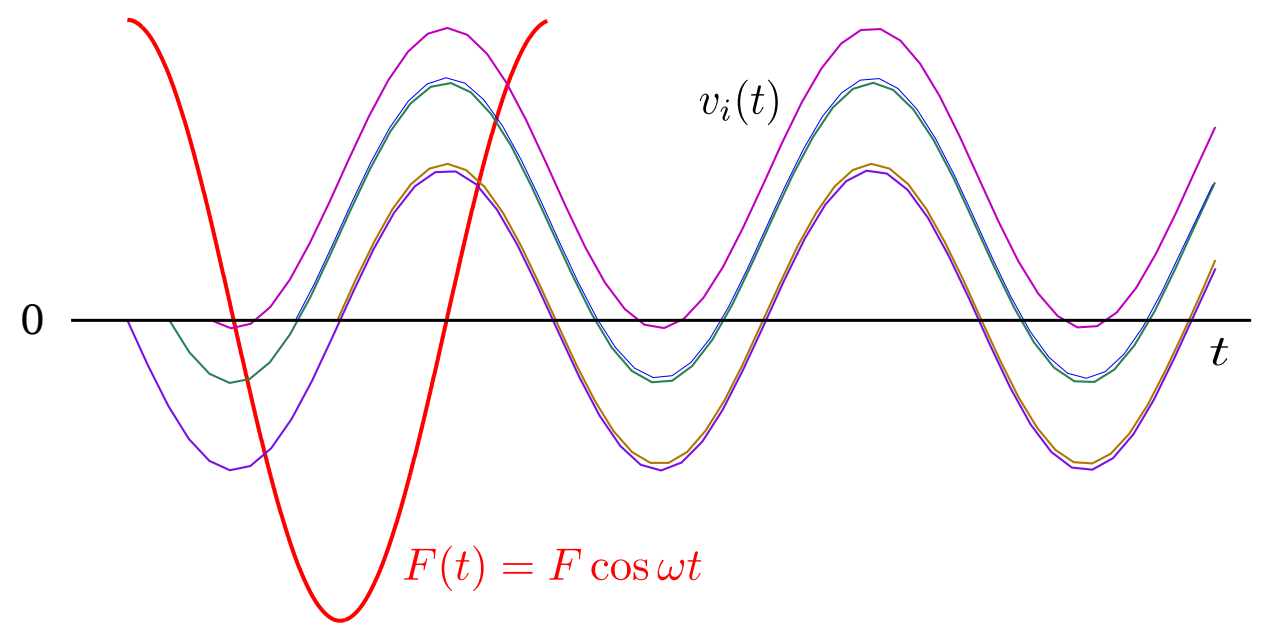

Figure 1.3: Parameters of the classical trajectories of an electron in the laser field $F(t)=F \cos \omega t$ after ionization at different times during the first half cycle of the laser oscillation. a) Coordinate $x_{i}(t)$ for $i$ trajectory, $b$ ) velocity $v_{i}(t)$ of an electron on $i$-trajectory. Parameters of calculation: laser wavelength $\lambda=800 \mathrm{~nm}$, intensity of the laser field $I=10^{14} \mathrm{~W} / \mathrm{cm}^{2}$.

After the end of the laser pulse, the electrons that have left the vicinity of the ion without returning to it will be detected as the so-called 'direct' photoelectrons. Few processes can happen when electrons on returning trajectories re-encounter the core: elastic scattering, inelastic scattering and recombina- 
tion with the parent ion, the latter accompanied by emission of radiation. It is this latter process that is responsible for the generation of high harmonics of the laser field, or high harmonic generation. As we are interested in HHG, we will be interested in the returning trajectories.

\subsubsection{Recombination step}

High energy photon is emitted after radiative recombination of the electron with the parent ion (figure 1.4). Its energy is given by the energy conservation law:

$$
\Omega=I_{p}+E_{\text {ret }}
$$

where $E_{r e t}$ is the energy of an electron at the moment of recombination $t_{r e c}$.

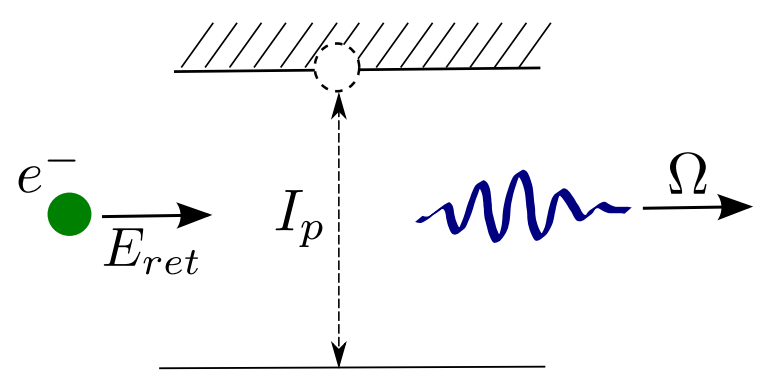

Figure 1.4: Emission of high energy photon with frequency $\Omega=I_{p}+E_{\text {ret }}$ after recombination of returning electron with energy $E_{\text {ret }}$ with the parent ion.

If we neglect the interaction of the electron with the ionic core, then its recombination energy is simply its kinetic energy at the moment of return (in atomic units):

$$
E_{r e t}=E_{k i n}=\frac{p^{2}}{2}
$$

Including the interaction with the ionic core typically introduces negligible changes to the total return energy compared with the simple model above. The reason is short interaction time compared to the laser cycle. As long as 
the laser field is nearly frozen during the electron return, the Hamiltonian can be treated as time-independent and the total energy is conserved.

Coordinate and velocity at a given time $t$ for a laser pulse with constant envelope and for a zero initial carrier-envelope phase of the field, according to 1.10 and 1.11, are:

$$
\begin{array}{r}
x(t)=\frac{F}{\omega_{L}^{2}}\left(\left(\omega_{L} t-\omega_{L} t_{i}\right) \sin \omega_{L} t_{i}+\cos \omega_{L} t-\cos \omega_{L} t_{i}\right), \\
v(t)=-\frac{F}{\omega_{L}}\left(\sin \omega_{L} t-\sin \omega_{L} t_{i}\right)
\end{array}
$$

It is convenient to make a substitution $\phi=\omega_{l} t$ and $\phi_{i}=\omega_{l} t_{i}$ :

$$
\begin{array}{r}
x(t)=\frac{F}{\omega_{L}^{2}}\left(\left(\phi-\phi_{i}\right) \sin \phi_{i}+\cos \phi-\cos \phi_{i}\right), \\
v(t)=-\frac{F}{\omega_{L}}\left(\sin \phi-\sin \phi_{i}\right)
\end{array}
$$

Recombination occurs when the electron returns to the zero coordinate (position of the ion) and recombination times can be found by solving equations $x\left(t_{r e c}\right)=0$ for each ionization time $t_{i}$. Solution for the equation $x\left(t_{r e c}\right)=0$ is presented in Figure 1.5. We immediately observe a distinct feature of electron trajectories that generate high harmonics: for the electron to return within one laser cycle after ionization, the ionization happens at times $0<\omega t_{i}<\pi / 2$, i.e. only during the first quarter of the laser cycle. Now we can express kinetic energy at any moment t:

$$
E_{k i n}=\frac{v^{2}(t)}{2}=\frac{F^{2}}{2 \omega_{L}^{2}}\left(\sin \phi-\sin \phi_{i}\right)^{2}=2 U_{p}\left(\sin \phi-\sin \phi_{i}\right)^{2}
$$

Using solutions 1.5 of the equation $x\left(t_{r e c}\right)=0$, we obtain the dependence of the energy of the emitted photon on the ionization time (magenta curve) or the time of recombination (black curve). They are shown in Figure 1.6. Ionization and recombination happen at different times within the laser cycle: trajectories are born during the first quarter of the cycle and they return during the next half period.

During the next half cycle of the laser wave, the sequence of ionization and 


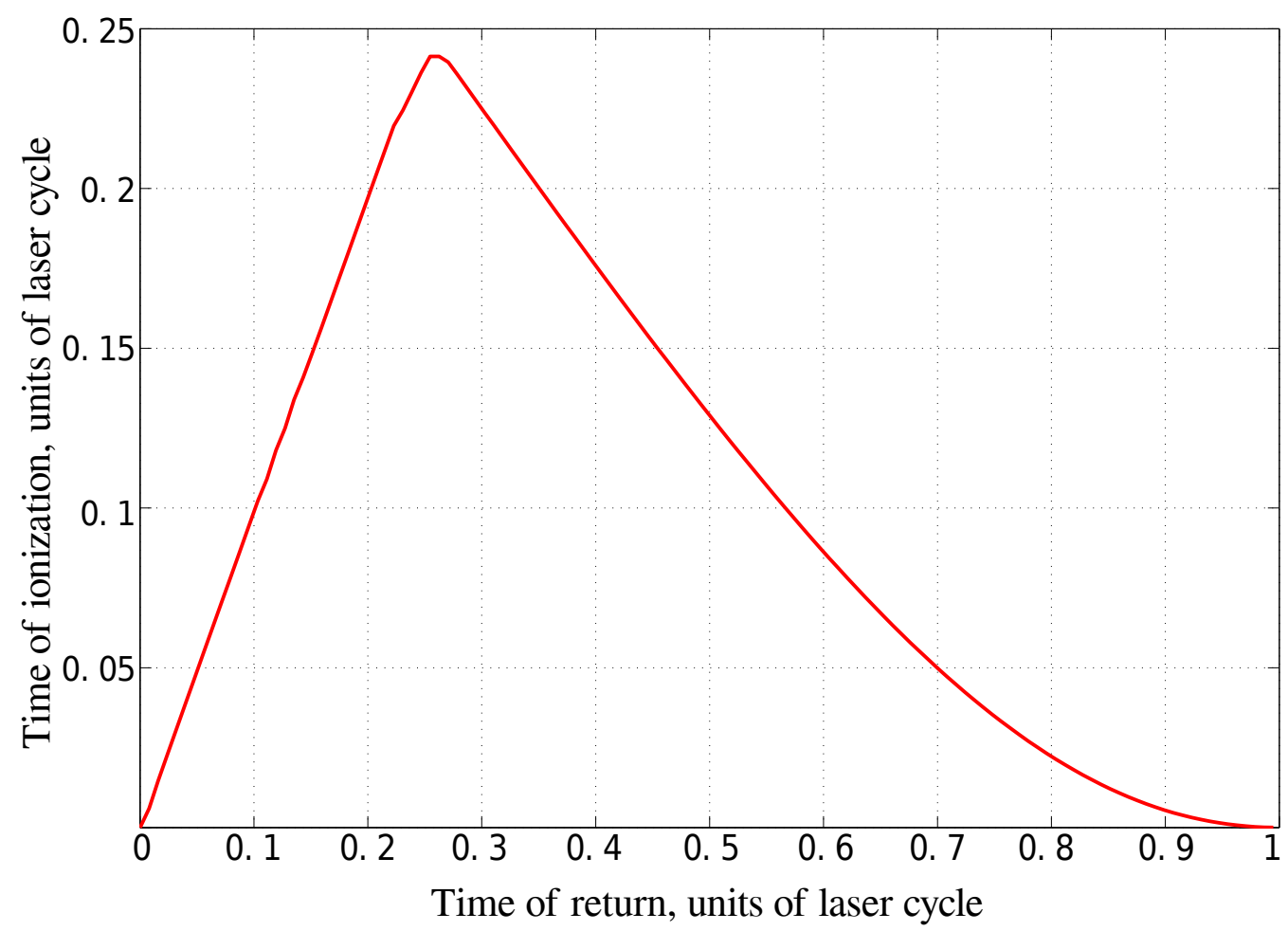

Figure 1.5: Dependence of the ionization time $t_{i}$ on the time of return $t_{r e c}$ in units of laser cycle. The part of the curve for $0<t_{\text {rec }}<0.25$ corresponds to contributions 'on the way out' of the barrier. Parameters of calculation: ionization potential $I_{p}=13.5984 \mathrm{eV}$, laser wavelength $\lambda=800 \mathrm{~nm}$, intensity of the laser field $I=10^{14} \mathrm{~W} / \mathrm{cm}^{2}$.

the following re-collision events happens again. The light emitted upon this sequence of ionization-recombination events interferes coherently with light emitted half a cycle ago. The same process repeats itself again and again during the following cycles of the laser pulse: we obtain a train of emission bursts repeating twice every laser cycle. As a result of the interference of the emission bursts within this train, the spectrum contains odd harmonics of the laser frequency, starting with the fundamental and extending to very high harmonic numbers, hence the name of the process - high harmonic generation. If the symmetry between half-cycles is broken in one way or another, it can lead to the generation of even harmonics. 


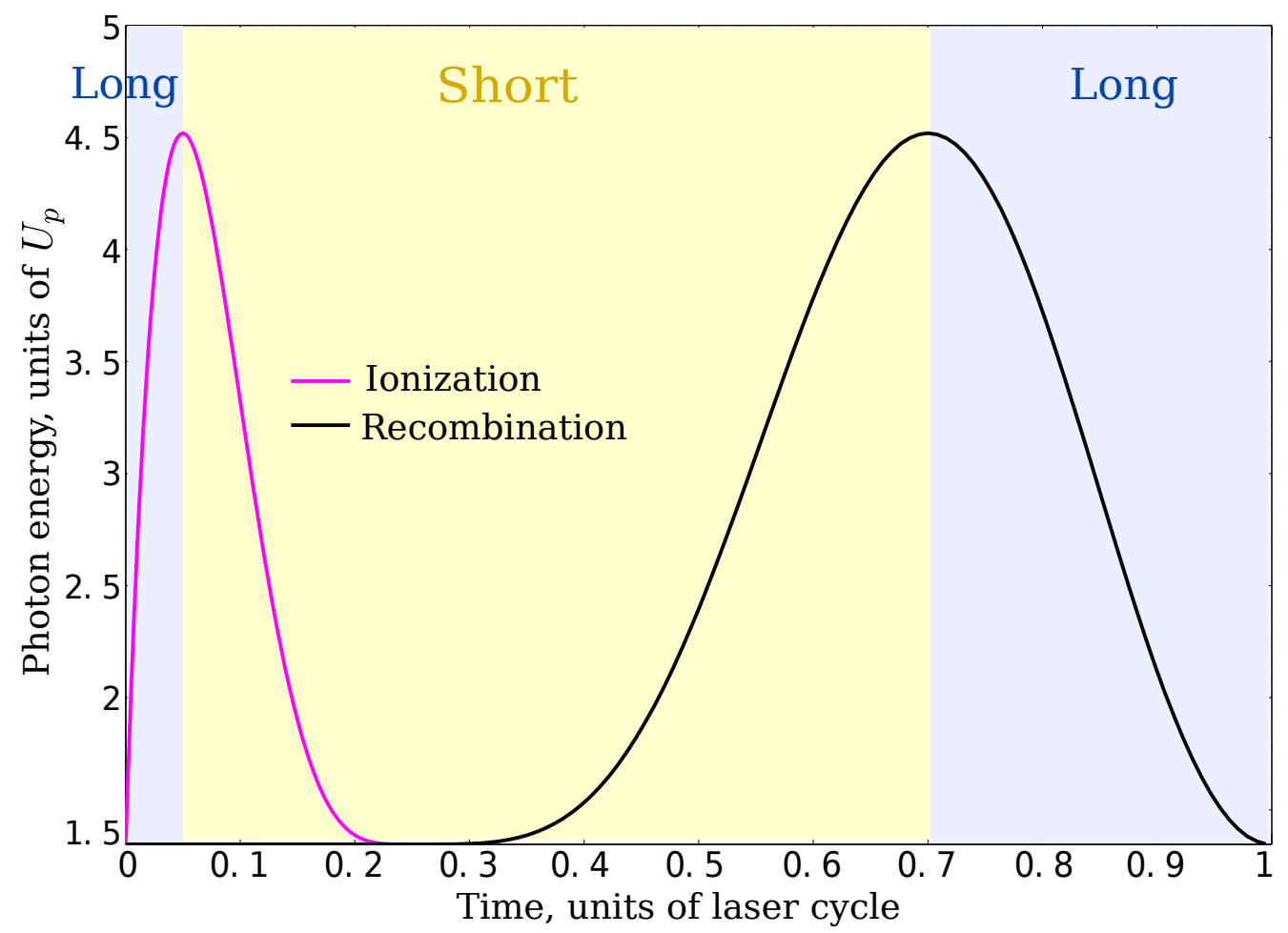

Figure 1.6: Photon energy $\Omega=E_{k i n}+I_{p}$ in units of ponderomotive energy $U_{p}$ $\left(\Omega / U_{p}\right)$. Magenta line shows dependence of $\Omega$ on ionization time, black line shows dependence of $\Omega$ on time of return. Parameters of calculation: ionization potential $I_{p}=13.5984 \mathrm{eV}$, laser wavelength $\lambda=800 \mathrm{~nm}$, intensity of the laser field $I=10^{14} \mathrm{~W} / \mathrm{cm}^{2}$.

A very important feature of the HHG spectrum is its cut-off: the characteristic emission energy beyond which the emission efficiency falls off exponentially. The origin of the cutoff can be understood by examining the energy curves in Figure 1.6, which shows that the electron return energy and, hence, the emitted photon energy, have a classical maximum. Classically, no emission can be generated beyond this maximum energy (beyond the cut-off). The universal law for the photon energy at the cut-off is $[48,49]$ :

$$
E_{c}=I_{p}+3.17 U_{p}
$$

The dependence of the return energy on the time of ionization and/or the return time is non-monotonic, see figure 1.6. For each return energy, except at the cut-off, there are two possible trajectories, that participate in the gen- 
eration of the same high harmonic photon. These trajectories are referred to as short and long trajectories. Short trajectories are born after the ionization time for the cut-off trajectory, and return before the return time for the cut-off trajectory (yellow area in Figure 1.6). Long trajectories are born before the cut-off trajectory and return after the cut-off trajectory (blue area in Figure $1.6)$.

Figures 1.5 and 1.6 show parameters of electron trajectories and photon energies within 1 period of the laser field oscillation $T=2 \pi / \omega_{L}$, in units of laser cycle. In many HHG experiments, the carrier wavelength of the laser is $\lambda=800 \mathrm{~nm}$. It means, that the period of the laser oscillation is:

$$
T=\frac{\lambda}{c}=\frac{800 \cdot 10^{-9} \mathrm{~m}}{3 \cdot 10^{8} \mathrm{~m} / \mathrm{s}}=2.7 \mathrm{fs}
$$

where fs stands for femtosecond, or $10^{-15}$ seconds. During these $2.7 \mathrm{fs}$, electron tunnels from at atom or a molecule, travels in the continuum and recombines with the parent ion. Thus, the whole process happens on the sub-femtosecond time scale, or the attosecond $\left(10^{-18}\right.$ seconds $)$ time scale. This fact provides the foundation for using the phenomenon of high harmonic generation to investigate atomic and molecular dynamics with attosecond resolution.

Observation of HHG signal may be considered as a pump-probe experiment, where the continuum electron probes its own parent ion (atom or molecule): ionization is a pump, time of electron excursion in the continuum is a pumpprobe delay, and the recombination is a probe of the molecular state at a certain point in time during the laser cycle. Each energy in the harmonic spectrum corresponds to a particular trajectory, and hence to particular ionization and recombination times. In this light, HHG spectrum can be regarded as a series of snapshots of the molecular dynamics at different times.

Electron has to return exactly back to the core in order to generate a harmonic photon. In the language of quantum mechanics, this corresponds to the maximum overlap between the returning electron wave packet and the state to which the recombination happens. Together with the angstrom-scale de Broglie wavelength of the returning electron, it gives rise to the angstromscale resolution encoded in the HHG spectra and to the opportunity for using 
HHG to investigate molecular structures ([73]).

Observing HHG spectrum under different experimental conditions and measuring such parameters as the amplitude, phase and polarization of the harmonic signal, one can unravel both the evolving structure (for example, [74-76]) and the complex electronic dynamics in a molecule or in an atom, with attosecond temporal and angstrom spatial resolution. The literature review in the next chapter will describe some of the noteworthy advances in this area of strong field physics. 


\section{Application of High Harmonic Generation to studies of atomic and molecular structure and dynamics.}

Attosecond physics is the area of atomic and molecular physics dedicated to the investigation of the electronically driven dynamics on its characteristic (attosecond) time-scale. High harmonic generation is a very powerful tool in the attosecond science toolbox.

First of all, HHG is a versatile source of coherent, bright, ultrafast light pulses in the broad range of photon energies, from VUV to XUV and soft Xrays. The HHG process results in a pulse train of coherent attosecond XUV pulses, and using short driving pulses it can yields an isolated attosecond pulse [68, 77]. This pulse can be used as a source of coherent XUV radiation for transient spectroscopy and attosecond streaking measurements [1-5, 78-80]. A lot of studies address the issue of creating an efficient table-top XUV source with special properties, for instance, controlled polarization or angular momentum $[38,81]$. Desired characteristics of an attosecond pulse are achieved via optimising phase-matching conditions, stabilising carrier envelope phase (CEP) [82-85], isolating a single attosecond pulse [86-91].

Implementation of the HHG signal as a source for spectroscopy is a rapidly developing field. However, this chapter will focus on another fruitful implementation of the phenomenon, namely, the use of HHG as a pump-probe scheme for the investigation of molecular dynamics. The amplitude and phase of 
HHG signal encode structural and dynamical information about the state of the system at different time points during the process ([73, 92-94]). Careful measurement and analysis of the parameters of harmonics provides an opportunity to reconstruct the dynamical picture of molecular processes. Below we will briefly review experimental and theoretical work that focus on obtaining both structural and dynamical information and the information about multielectron effects, including the exciting advances of HHG in multicolor fields and its application for tracking ultrafast molecular dynamics.

\subsection{Structural information}

\subsubsection{High harmonic Generation in atoms}

The process of generating high harmonic light depends on the ionization and recombination properties of studied particles, atoms or molecules. Since atoms are isotropic systems, their ionization does not depend on the angle of the laser polarization with respect to a chosen fixed direction in the atom. Electron propagation depends weakly on the properties of the ionic core, and is dominated by the properties of the laser field. In the 3 -step model, the weak dependence of the electron trajectory on the neutral system arises through the dependence of the ionization time on the tunneling step, i.e. on the tunnelling properties of the atom in question, for given laser field parameters. The strongest dependence on the quantum system comes via the last - recombination - step. Indeed, recombination strongly depends on the state of the ion in which the system is left after tunnelling. Different atoms have different orbital wave functions and very different recombination matrix elements, which encode the information about the atomic states involved.

Several investigations have been done on the structure of the atomic ground state via high harmonic generation. It was shown that differences in the harmonic spectra for different rare gases are clearly linked to the differences in the orbital wave function during the recombination step [95] and reflect the photorecombination cross sections, which are the same as the one-photon ionization cross sections. 
In various experiments, different characteristic features of atomic photo-ionization were observed in the harmonic spectra (i.e. via the photo-recombination step). For instance, the Cooper minimum [96] was observed for $\operatorname{Ar}$ [97] and $\operatorname{Kr}$ [98].

\subsubsection{Molecular alignment}

In contrast to atoms, which are isotropic, we find very different situation in molecules, which are anisotropic systems. Ionization and recombination can strongly depend on the alignment angle. Thus, HHG process must reflect this dependence. Crucially, modern HHG experiments can study aligned molecules. Below we briefly describe the way alignment is achieved.

A molecular gas under normal conditions is an ensemble of randomly aligned molecules, with equally probability to find a molecule aligned in any given direction. However, in many HHG experiments the generating molecules are aligned.

To understand how the molecular alignment takes place, let us consider a diatomic molecule without a permanent dipole moment (e.g. a homonuclear diatomic). If, for instance, a linearly polarized non-resonant laser field is applied to an ensemble of such molecules, the electrical field of the laser $\mathbf{E} \cos \omega t$ will induce a dipole moment $\mathbf{p}(\mathbf{E})$ in each molecule. The potential energy of this interaction is:

$$
U=-\int_{0}^{\mathbf{E} \cos \omega t} d \mathbf{E}^{\prime} \mathbf{p}\left(\mathbf{E}^{\prime}\right) \mathbf{E}^{\prime}
$$

The dipole moment $\mathbf{p}(\mathbf{E})=\hat{\alpha} \mathbf{E}$ depends on the polarizability of the molecule, with $\hat{\alpha}$ the polarizability tensor, e.g. in Cartesian coordinates $(x, y, z)$. Generally speaking, the polarizability of a molecule is anisotropic and can induce complex response to the applied laser field due to the properties of the polarizability tensor. This dependence of the molecular response on the direction of the applied electric force leads to the same dependence of the potential energy of the interaction and makes the molecular alignment possible. In our simple case of a linear molecule, the induced dipole moment is $p_{\|, \perp}=\alpha_{\|, \perp} \times\{\cos \theta, \sin \theta\} E \cos \omega t[99]$, where $\theta$ is the Euler angle between 
the molecular axis and the electric field vector of the laser pulse, and $\alpha_{\|, \perp}$ are the polarizabilities parallel and perpendicular to the molecular axis. The expression for the interaction energy becomes:

$$
U=-\frac{1}{2}\left[\Delta \alpha \cos ^{2} \theta+\alpha_{\perp}\right] E^{2} \cos ^{2} \omega t
$$

where $\Delta \alpha=\alpha_{\|}-\alpha_{\perp}$. After the averaging over the laser cycle period and dropping the isotropic part of the interaction energy, we arrive to the expression [99-101]:

$$
U(\theta)=-\frac{1}{4} \alpha E^{2} \cos ^{2} \theta
$$

As we can see from the formula above, the electrical component of the applied laser field introduces the force $F=-\partial U(\theta) / \partial \theta$ which turns the molecule along the polarization of the field, where it minimizes the potential energy of the interaction.

The ensemble of molecules in thermodynamic equilibrium contains molecules oriented in all directions. This changes as the aligning interaction potential $U(\theta)$ is introduced, leading to the alignment distribution $P(\theta)$. A useful measure for the degree of molecular alignment is the ensemble-averaged $\cos ^{2} \theta$ :

$$
\left\langle\cos ^{2} \theta\right\rangle=\frac{1}{2} \int_{0}^{\pi} P(\theta) \cos ^{2} \theta \sin \theta d \theta
$$

For molecules with random alignment we obtain $\left\langle\cos ^{2} \theta\right\rangle=\frac{1}{3}$. The same calculation will give us $\left\langle\cos ^{2} \theta\right\rangle=1$ for perfect alignment at $\theta=0$ and $\left\langle\cos ^{2} \theta\right\rangle=0$ for molecules aligned in the plane orthogonal to the laser polarization, i.e. at $\theta=\frac{\pi}{2}$.

Quantum mechanical description of the interaction between ensemble of molecules and an aligning laser pulse is described in [99-101]. Adiabatic alignment of molecular rotational states happens if the laser pulse is long compared to the rotational periods of the states in question [101]. In this case the eigenstates of the aligned molecules are the so-called pendular states, that can also be understood in classical representation [99, 101, 102]. Each pendular state is characterized by the total angular momentum number $J$ and its projection on 
the fixed axis $M$ [101]. These quantum numbers and these states adiabatically connect to the field-free rotational state $J, M$.

Molecular alignment also happens in the opposite, impulsive limit, when the length of the aligning pulse is much shorter than the rotational period of the quantum state. In this case, the laser pulse gives a kick to the molecular ensemble towards alignment and the evolution of this ensemble as a function of $\theta$ happens after the end of the pulse, leading to transient alignment after the end of the aligning pulse [99]. The long-term, purely laser field-free evolution, is determined by the structure of the rotational spectrum $E_{J}=B J(J+1)$, where $B$ is the rotational constant of the molecule. Importantly, the spacings between adjacent rotational states are all integer of $2 B$, meaning that the evolution of the rotational wavepacket is periodic with the period $\pi / B$. Thus, periodic revivals of the prompt alignment after the laser pulse occur.

The Boltzmann thermal distribution of molecular rotational states is also taken into consideration. Thus, the measure of the alignment in the given ensemble is $[101,103,104]$ :

$$
\begin{array}{r}
\left\langle\cos ^{2} \theta\right\rangle=\sum_{J} w_{J} \sum_{M=-J}^{M=+J}\left\langle\cos ^{2} \theta\right\rangle_{J, M}, \\
w_{J}=\frac{1}{Q_{r}} e^{-J(J+1) \frac{B}{k T}}
\end{array}
$$

where $B$ is the rotational constant of the molecule, $Q_{r}$ is the rotational partition function, $\left\langle\cos ^{2} \theta\right\rangle_{J, M}$ is the mean value of $\cos ^{2} \theta$ for rotational state $\{J, M\}$. 

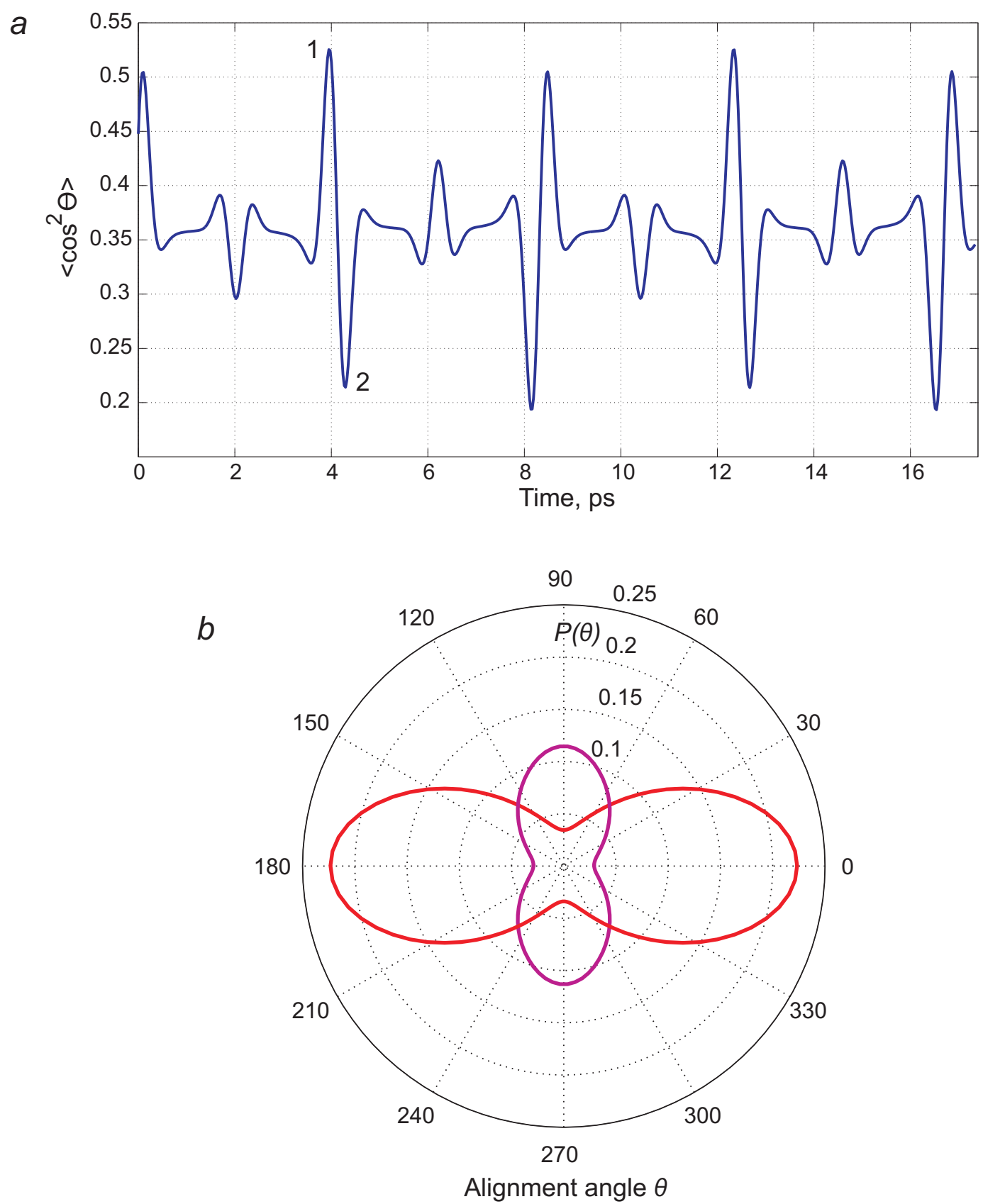

Figure 2.1: Rotational revivals of the $N_{2}$ molecule for rotational temperature $T=50 K: a$ ) peaks of $\left\langle\cos ^{2} \theta>\right.$ represent 1 - alignment (along the laser polarization) and 2 - anti-alignment (perpendicular to the laser polarization). b) alignment distribution of aligned molecules (red) at the moment 1 on the plot above and anti-aligned (magenta) at the moment 2 on the plot above [105]. 
The resulting evolution for typical experimental conditions, for the nitrogen molecule, is shown in Figure 2.1, generated with the code in [105]. For each molecule, at the start of the process a state $\{J, M\}$ is populated. Interaction with the ultrashort aligning pulse populates many adjacent states. The rotational wavepacket propagates freely after the laser pulse is turned off. The phase evolution of many rotational states leads to their periodic phasing and de-phasing, referred to as rotational revivals. The results presented in Figure 2.1 are averaged over the incoherent ensemble of initial states populated according to the Boltzmann distribution. During this evolution $\left\langle\cos ^{2} \theta\right\rangle(t)$ will have regular maxima and minima, which mean that the distribution is aligned along laser polarization (maxima) or in the plane orthogonal to it (minima). The width of the peaks depends on the rotational temperature of the ensemble and the degree of coherence induced between the excited states by the aligning pulse.

Importantly, typical laser pulses with duration of about $100 \mathrm{fsec}$ and peak intensity of some $10^{13} \mathrm{~W} / \mathrm{cm}^{2}$ are very efficient in aligning molecules. The typical depth of the aligning potential well can easily reach $50 \mathrm{meV}$ without ionizing or exciting the molecule vibrationally. This is a very strong aligning potential, given that the thermal energy at the room temperature is only about $25 \mathrm{meV}$. Thus, high degree of molecular alignment is possible, especially using molecular cooling as they are expanded into the vacuum through a nozzle of a jet, under pressure [99].

\subsubsection{High harmonic generation in aligned molecules: General features}

In molecules, ionization and recombination strongly depend on the alignment angle. Comparison of the harmonic spectra even for unaligned molecules and atoms with very close ionization potentials shows the difference in the structure of the spectra and in the position of the harmonic cutoff [106-108]. The difference appears already due to the properties of the outermost orbitals. Similar study in alkanes [109] clearly showed the difference in the structure of the HHG spectrum for alkanes and Xe, and graphically demonstrated the 
insufficiency of the SAE approximation with a single hydrogenic orbital for the description of the experiment in complex molecules such as alkanes.

The first quantum mechanical description of the ionization dependence on the full range of angles was presented in [110]. There, ionization of the molecular hydrogen in a strong low-frequency field was studied, specifically, the dependence of the ionization rates on the internuclear distance for states with different symmetry, and also the dependence on the angle between the molecular axis and the laser polarization vector. As a consequence of these dependencies, the high harmonic generation process should also depend on the molecular alignment angle and on the properties of the ionizing orbital, from which the electron leaves the molecule.

The first observation of HHG in laser-aligned molecular ensemble was made in [103], and further in [111]. For the first time, laser-induced alignment was achieved at relatively high densities of the molecular gas, $\sim 10^{17} \mathrm{~cm}^{-3}$, which is a key prerequisite for HHG. The molecules studied experimentally and numerically were $\mathrm{CS}_{2}$ and $\mathrm{N}_{2}$. Both experimental and simulated results showed strong dependence of the signal on the molecular alignment angle. Increase in intensity of the HHG signal when the generating pulse was preceded by the alignment pulse, relative to the signal without the alignment pulse was shown for $\mathrm{CS}_{2}$ molecules. Suppression of the signal for the $\mathrm{N}_{2}$ molecules aligned perpendicularly to the HHG generating pulse was also found.

Multiple further studies showed, that HHG spectrum in aligned molecules is strongly affected by the structure of the molecular orbitals, involved in the process. The alignment dependence of HHG in the carbon dioxide molecule was studied for the first time in [112]. The maximum yield of harmonics was found to be strongly dependent on the harmonic number at intermediate angles between $0^{\circ}$ and $90^{\circ}$, with a minimum in the yield at $\theta=0^{\circ}$. The overall minimum at $\theta=0^{\circ}$ is consistent with the symmetry of the highest occupied molecular orbital of $\mathrm{CO}_{2}$. The latter has a nodal plane in the $\theta=0^{\circ}$ direction. The presence of the nodal plane suppresses both ionization and recombination amplitudes.

The influence of the symmetry of the molecular orbital on the HHG was then shown in $[113,114]$ via theoretical comparison of $\mathrm{HHG}$ in aligned $\mathrm{N}_{2}$ and $\mathrm{O}_{2}$ 
molecules, that are characterized by different symmetry of the highest occupied molecular orbital: $\sigma_{g}$ for the $\mathrm{N}_{2}$ and $\pi_{g}$ for the $\mathrm{O}_{2}$. Experimental confirmation of different harmonic modulation depending on the alignment angle, for $\mathrm{N}_{2}$ and $\mathrm{O}_{2}$, is presented in [115].

As the next step in the investigation of the molecular orbital dependence of HHG, more complex molecules were examined. Polyatomic molecules such as allene and acetylene were studied in [116]. The expansion of this study is presented in[117], where additionally HHG in aligned ethylene was observed. Experimental results were found to be in a good agreement with theoretical calculations based on the strong field approximation (SFA), showing strong dependence of the harmonic yield on the molecular alignment angle. With changing the alignment angle HHG spectrum is modulated for all harmonic numbers, but the modulation is not the same for different harmonics. This is a characteristic feature of HHG in aligned molecules. It is a manifestation of the dependence of the recombination dipole on the energy of the returning electron.

Ionization potentials of some of the small molecules like $\mathrm{CO}_{2}, \mathrm{~N}_{2}$ or $\mathrm{O}_{2}$ are relatively high $(\sim 13 \mathrm{eV})$, or, in other words, the number of obtained harmonics for the values of the pump laser parameters, typically used in experiments, is sufficient to study HHG across a broad range of the harmonic photon energies. However, most larger molecules, such as those investigated in [116] and [117], have smaller ionization potentials $I_{p} \sim 9-10 \mathrm{eV}$. This significantly limits the driving laser intensity that can be applied to the molecule before it is fully ionized, keeping the intensity well below $10^{14} \mathrm{~W} / \mathrm{cm}^{2}$ and dramatically reducing the position of the HHG cutoff $\Omega_{\max } \simeq I_{p}+3.2 U_{p}$.

This problem can be resolved by increasing the wavelength $\lambda$ of the driving laser, since $U_{p} \propto \lambda^{2}$. The constraint is the wavelength-dependence of the HHG efficiency. The electron return probability is reduced with growing $\lambda$ due to the large transverse spread of the re-colliding electronic wavepacket. The wavepacket spreading between ionization and recombination scales linearly with the laser period for each of the three spatial dimensions, leading to $\lambda^{-3}$ drop in the signal due to the spreading alone. Combined with $\lambda^{2}$ growth of the harmonic spectral width and $\propto \lambda$ growth in the density of the harmonic 
lines, this leads to the overall $\lambda^{-6}$ decrease in the harmonic signal, for a singlemolecule response [118]. This signal loss can be partially offset by increasing the density of the molecular gas and by optimizing the phase-matching conditions, see e.g. [119] and references therein for discussion.

These conditions were accounted for in the experiment [120], where optimal laser wave length was found to be around $1300 \mathrm{~nm}$ (and up to $1800 \mathrm{~nm}$ ) for molecules $\mathrm{N}_{2}, \mathrm{CO}_{2}, \mathrm{C}_{2} \mathrm{H}_{2}$ and $\mathrm{N}_{2} \mathrm{O}$. An extended study of $\mathrm{CO}_{2}$ at the longer wavelengths and different intensities of the driving laser was done later in the work [121], which is considered later in chapter.

\subsubsection{High harmonic generation in aligned molecules: Structural features of Molecular Orbitals}

In order to understand the influence of the geometrical structure of the ionizing molecular orbital on the HHG we should consider its role during each step of the HHG process.

Ionization depends on the direction of the ionizing field polarization via the dependence of the ionization (tunnelling) rates on a particular direction in the molecule [110]. As it was mentioned in the previous section, the propagation step (almost) does not depend on the molecular alignment directly, for sufficiently large electron oscillation amplitude $F / \omega_{L}^{2}$, compared to the molecular size. How is the recombination influenced by the direction of the driving laser polarization with respect to the molecular axis and the geometry of the electronic wave function of ionic orbital to which the returning electron recombines?

To answer this important question, let us consider HHG in an aligned diatomic molecule, as it was done in the theoretical work with molecular hydrogen $\mathrm{H}_{2}$ and its ion $\mathrm{H}_{2}^{+}$[122]. In this study, a pronounced minimum in the harmonic spectrum was found. This minimum was fixed for varying intensities for a certain alignment direction, but it's position moved along energy axis when changing the alignment angle. Implementing 2D models of the ion and 
molecule and solving the time-dependent Schroedinger equation (TDSE) numerically for the softened Coulomb interaction between the charged particles, it was shown, that the minimum is accompanied by the dramatic change of the harmonic phase in its vicinity. Remarkably, this interference is found to be the signature of structure of the orbital and not dependent on the parameters of the driving laser pulse.

Destructive interference in a diatomic molecule, leading to the minimum in HHG spectrum can be considered as result of the intra-molecular interference between the two emitting centers, and has its counterpart in photo-ionization discussed by Cohen and Fano in 1960-th. In HHG, this idea was first introduced in [123, 124], studying, again, the example of the molecular hydrogen ion. The recolliding electron is approximated with the plane wave with the de Broglie wavelength $\lambda$. In this case that the conditions for constructive and/or destructive interference in recombination to the two centers (two-center interference) are [123]:

$$
\begin{gathered}
R \cos \theta=(2 m+1) \lambda / 2, \\
R \cos \theta=m \lambda, \\
m=0,1,2, \ldots \ldots
\end{gathered}
$$

Here $R$ is internuclear distance, $m$ is the order of the minimum or maximum. Top equation in 2.7 is a condition for the destructive interference and the lower equation is for the constructive interference. Note that the basic interference occurs in the electronic transition and not in the emitted light in a sense that the positions of the minima and maxima are determined by the electron de Broglie wavelength and not by the wavelength of the emitted light.

The structure-related destructive minima are often observed in experiments of HHG in molecules. The main characteristic of this type of minimum in the harmonic spectrum is that, for a fixed alignment of the molecule, it is observed at the same energy independent of the laser parameters such as the laser wavelength and/or intensity. As pointed out in [123], the maxima are not usually observed in experiments, because their positions along the energy axis are four times higher in energy than the position of the minimum, so that 
the maximum is normally situated beyond the cut off.

The confirmation of the model calculation in the molecular hydrogen in [123, 124] was observed in the experimental and theoretical work on $\mathrm{CO}_{2}$ [125], where the concept of the two centers for electron recombination was extended to the linear $\mathrm{CO}_{2}$ molecule, consisting of three atoms. The structural minimum of the $\mathrm{CO}_{2}$ molecular orbital moved with the alignment angle and the spectrum of aligned molecules was compared to that of unaligned molecules.

Further evidence of the intermolecular interference in $\mathrm{CO}_{2}$ and additionally $\mathrm{N}_{2}$ was provided in the work [126], where harmonic spectra of the aligned molecules were compared to the spectra from the molecular ensemble with random alignment distribution. Ion yields were also measured during the experiments, which allows one to calibrate the effect of the ionization step in each case. The interplay between the conditions for the maximum ionization probability and the recombination probability leads to a counter-intuitive result for $\mathrm{CO}_{2}$ : when ionization is enhanced $\left(\theta=30^{\circ}\right)$, destructive interference in recombination step leads to the harmonic yield lower than for unaligned molecules.

\subsubsection{Molecular tomography with high harmonic generation}

Systematic investigation of HHG in aligned molecules and observation of signatures from molecular orbitals in the spectrum leads to an idea of the orbital reconstruction from the experiment. The molecular orbital tomography technique - imaging of the highest molecular orbital using HHG spectrum of aligned molecules - was first proposed and implemented in [92] for HOMO reconstruction in $\mathrm{N}_{2}$.

The harmonic spectrum of molecular nitrogen was measured for 19 angels from $0^{\circ}$ to $90^{\circ}$ with $5^{\circ}$ interval. The HHG spectrum of the Argon atom, which has nearly the same ionization potential as $\mathrm{N}_{2}$ and hence very similar ionization and propagation steps, was used as a reference for calibrating the continuum wave packet and the HHG spectrum, normalizing out the contributions from the first two steps of the HHG process. The tomographic procedure assumes that the continuum electron dynamics in the strong laser field can be approximated 
with the Volkov states (the solutions for the free electron in the laser field), which spatially correspond to plane waves. Hence, the recombination step is approximately proportional to the Fourier transform of the orbital involved in recombination. The reconstruction procedure then exploits the 2D Fourier slice theorem, stating that the Fourier transform of the projection of the function on a certain direction is equal to the slice of the Fourier transform of that function in that direction. In the molecular tomography terms, the FT of a recorded spectrum at an angle $\theta$ is a cut of the orbital at $\theta$ alignment. The results of the reconstruction are in good agreement with the $a b$ initio calculation of the HOMO orbital. Furthermore, the imaging technique, suggested in [92] opens a possibility for reconstructing electron dynamics in the molecule during the laser cycle. This dynamics happens due to the coherent population of more than one ionic state states upon ionization, i.e. with several orbitals involved in the ionization and recombination steps [127], see discussion later.

Analysis of the tomographic procedure [128] showed, that the amount of obtained harmonics in the plateau region is not enough for the accurate reconstruction of the molecular orbital's wave function, essentially due to the frequency restricted Fourier spectrum. Producing HHG spectrum with the longer wave lengths can improve the results due to significant increase of the cutoff energy and, consequently, higher resolution of the FT. The internuclear distances and symmetry of the HOMO can be extracted with good accuracy, which was shown with the examples of $\mathrm{N}_{2}, \mathrm{O}_{2}$ and $\mathrm{CO}_{2}[128,129]$.

Described tomographic procedure is based on the approximation of the returning electron wave packet as a superposition of plane waves. Improvement over this assumption is suggested in [121], where the re-colliding wave function is presented as plane wave with first order perturbation correction of the ionic field $U(\mathbf{r})$. HHG spectrum of aligned at $0^{\circ} \mathrm{CO}_{2}$ was obtained for three wavelengths in the near-IR range: 1480, 1600 and $1700 \mathrm{~nm}$. For each wavelength, the intensity scans were performed, recording the spectra for 4 different intensities. Minimum at about $60 \mathrm{eV}$ was observed for all spectra, which was associated to the structure of the HOMO. Corrected molecular tomographic procedure reconstructed the $\mathrm{HOMO}$ of $\mathrm{CO}_{2}$, showing good agreement with the theoretical calculations of the orbital, obtained with quantum chemistry 
methods. The differences between the theoretical and the reconstructed results were attributed to the limited range of frequencies, available for the FT.

\subsection{Nuclear and electronic dynamics resolved via High Harmonic Generation}

\subsubsection{Nuclear dynamics via HHG}

Recollision of an electron with the parent ion after ionization in a strong IR field is a very precisely timed process. It can therefore serve as a basis for the temporal attosecond resolution of the dynamics happening in the ion during the laser cycle. This idea was put as a basic principle in the work [130, 131], where the dynamics of the dissociation and the molecular vibration induced by ionization was investigated through the observation of the kinetic energy of the inelastically scattered electrons and NSDI process.

The same principle underlies numerous works studying molecular rotational, vibrational and dissociation dynamics with HHG. Only the observable in these experiments is different, namely, the light emitted as a result of the HHG process. The possibility of dissociation studies with HHG were discussed e.g. in $[132-134]$.

Thorough theoretical studies [135-138] for $\mathrm{H}_{2}$ and $\mathrm{D}_{2}$ hydrogen isotopes and [139] for $\mathrm{H}_{2} \mathrm{O}$ and $\mathrm{D}_{2} \mathrm{O}$ water molecules predicted that the HHG signal has to have signatures of slower or faster vibrational motion for different isotopes, and that the very early stages of the vibrational dynamics can be reconstructed from the HHG signal, with sub-femtosecond resolution. These predictions were confirmed by a series of experiments in isotope pairs such as $\mathrm{H}_{2} / \mathrm{D}_{2}$ and $\mathrm{CH}_{4}$ and $\mathrm{CD}_{4}[74,140]$, which measured the relative harmonic intensities for each pair. The interpretation of the HHG as a pump-probe scheme, where ionization is represented as a pump and the recombination as a probe, is exploited in these studies: each harmonic via its amplitude, polarization and phase contains information about the nuclear dynamics of the molecule. 
A different pump-probe scheme was implemented in experiment [75], where the vibrational wave packet in $\mathrm{SF}_{6}$ molecule was first excited via an impulsive stimulated Raman process. The delayed probe pulse then generated high harmonics. Similar oscillations in the harmonic yield were detected for all harmonic numbers as a function of the pump-probe delay. These oscillations were attributed to the vibrational motion of the molecule. Vibrational periods were found via the Fourier transform of the harmonic yield oscillations and agreed with known vibrational modes of the molecule.

Similar experiment were performed in the gas of the $\mathrm{NO}_{2}$ dimers - the molecule $\mathrm{N}_{2} \mathrm{O}_{4}$ [141]. A weak pump beam excited the ground vibrational state of the dimer, while the probe strong IR pulse induced HHG. Again, the oscillations of the harmonic yield, depending on the pump-probe delay, were measured. Theory of probing large amplitude vibrations in molecules, that incorporates the modification of the vibrational dynamics by the probe field, was developed in [142] and it described many features of the experiment in $\mathrm{N}_{2} \mathrm{O}_{4}$ dimer[141].

Experiments in aligned molecules $[115,125,143,144]$ investigated the rotational excitation dynamics of $\mathrm{O}_{2}, \mathrm{CO}_{2}$ and $\mathrm{N}_{2}$ in a pump-probe scheme as well. The harmonic intensity dependence on the delay between the alignment pulse (pump) and the high harmonics generating pulse (probe) reproduced the characteristic patterns of the rotational revivals. In a similar work with $\mathrm{CO}_{2}$ and $\mathrm{N}_{2} \mathrm{O}$ [76], apart from the investigation of rotational revivals, the electron was shown to gain angular momentum from the laser field during its excursion in continuum. Introduction of the additional control parameter, specifically, the polarization angle between the alignment pump pulse and the generating probe pulse [145], has allowed the authors to increase the sensitivity of detecting rotational revivals. The contrast between the harmonic yield at the moment of the rotational revival and away from the revival was raised by the factor of 4, when the angle between the pump and probe pulses was introduced (and controlled). Although it provided significant sensitivity improvement in the observation of the revival peak, it was achieved at the cost of the overall 30 times intensity drop in the harmonic signal, in comparison to the parallel configuration of the pulses.

Investigation of the oriented rotational wave packet via the observation of even- 
order harmonics [146] demonstrated the mechanism of orientation by selective ionization of polar molecules, using the example of CO. The combination of $\omega_{L}$ and $2 \omega_{L}$ driving fields introduces high asymmetry between the peak electric field strengths during the subsequent half-cycles of the laser field, consistently preferring one direction along the laser polarization axis relative to the opposite one. Polar CO molecules are preferentially ionized along the direction corresponding to ionization from the carbon-side of the molecule. Thus, oriented (by bleaching) neutral ensemble of $\mathrm{CO}$ molecules is created together with oriented ions. Because of the high asymmetry in the system, even harmonics are observed and the orientation dynamics can now be studied with the pump-probe scheme, as described above.

\subsubsection{High harmonic generation: multielectron effects}

So far we have considered experimental work in HHG, which could be described by taking into account the dynamics of only one electron and assuming that only the highest occupied molecular orbital contributed to the HHG signal. Is it possible to probe multi-electron dynamics with high harmonic generation and reach lower lying molecular orbitals?

Ionization probability drops with exponential speed as we increase the binding energy of the orbital, but in molecules the orbitals are close to each other, with several orbitals within a couple of $\mathrm{eV}$. Different channels of ionization can thus be expected to play role, as well as the electron-electron correlations $[147,148]$. Furthermore, the interplay between the structural properties of the orbitals at different angles allows for seemingly less likely channels to manifest themselves in the harmonic spectra [127]. We will consider the pioneering essential works in the study of the multielectron effects in the HHG spectrum.

Works on the role of electron exchange and spin statistics of electrons in the molecule $[147,148]$ showed that molecular orbital tomography, first described in [92], reconstructs the Dyson orbital of the molecule together with the effects of the electron correlations, imaging signatures of lower orbital as well as HOMO. Dyson orbital is an overlap between the wave function of the ground 
state of the neutral and the electronic wave function of the ion. The language of Dyson orbitals is very convenient for the description of molecular ionic states.

Thorough experimental and theoretical investigation of the contributions from different orbitals into the resulting spectrum was done in [127] by studying the $\mathrm{HHG}$ signal of $\mathrm{CO}_{2}$ molecule from different alignment angles. Electron can leave the ion in the ground state or in one of the excited states after ionization. Harmonic generation, that happens through one of these states, represents one channel of the process. It was rigorously shown, that the observed harmonic spectrum is the result of the interference from multiple channels of HHG. Schematic illustration of the multichannel principle is shown in Figure 2.2, adapted from the original paper [127].
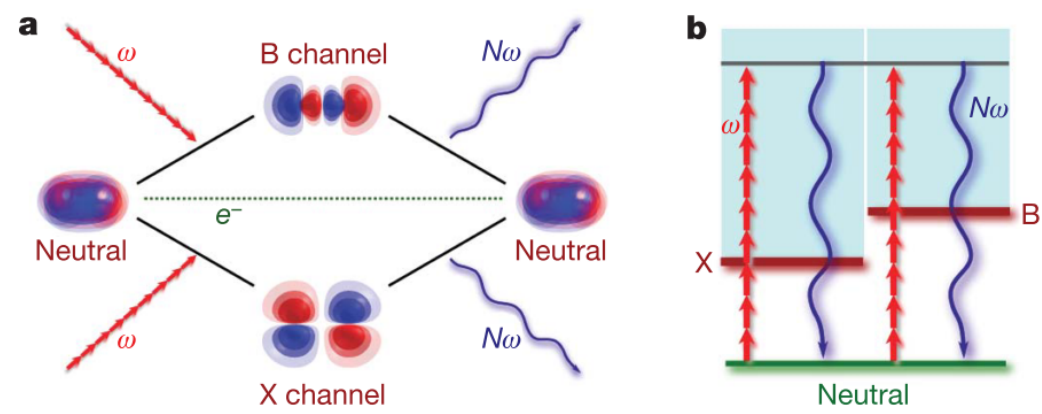

Figure 2.2: Illustration of the interference between different channels in high harmonic generation. Adapted from [127].

Lower lying orbitals reveal themselves especially clearly when HOMO has weak contribution to the resulting spectrum. The analysis of the HOMO and HOMO-2 recombination matrix elements of $\mathrm{CO}_{2}$ and its harmonic spectrum at different alignments demonstrated, how the interference between different channels of the process takes place: the HOMO-2 takes over the spectrum, when the HOMO contribution is suppressed. This happens near the cutoff region where higher $I_{p}$ gives preference to the HOMO-2 contribution, or in the vicinity of the structure-related minimum in the HHG from HOMO. Their dynamical interference manifests itself in the shift of the interference minimum in the spectrum with intensity of the driving laser. Interference between channels 
of HHG has a dynamical nature unlike the stationary structural interference, because the phase at which the destructive interference occurs is associated with a certain time of excursion in the continuum [127]. The same time of travel corresponds to different harmonic energies, when the intensity changes: the higher the intensity, the higher the energy at which the destructive interference takes place.

The study of the dynamical interference in $\mathrm{CO}_{2}$ at longer wavelength $\lambda=1140$ $\mathrm{nm}$ (unlike $800 \mathrm{~nm}$ in the previous study [127]) was done in [94], where the shift of the destructive interference phase with the laser wavelength was shown. For different driving laser wavelengths, the relative recombination phase between channels will change, leading to the shift in the position of the minimum, even for the same travel time. As it was pointed out in [94], the change of the recombination phase is dramatic near the structural minimum of one of the orbitals and in these regions the dynamical interference will have the strongest dependence on the laser parameters.

Further important step in uncovering the multielectron dynamics in molecules in strong laser fields is the inclusion of the sub-cycle dynamics in the molecular ion during the travel of the liberated electron in the continuum. After ionization, several ionic states can be populated, which starts the dynamics in the ion. This dynamics was included in both theoretical calculations in [94, 127], showing that for longer wavelengths the real population transfer between different ionic states is less significant. Furthermore, the study of the dynamical interference in nitrogen molecule $\mathrm{N}_{2}$ in [149] showed the crucial role of the sub-cycle laser-induced dynamics in the ion for reproducing the experimental results.

\subsection{Multicolor schemes for high harmonic spectroscopy}

As we have already described above, the high harmonic generation spectrum contains information about both the nuclear and the electronic dynamics and the structure of the molecules. The challenge of experimental and theoretical 
studies is to interpret the experimental results, disentangling the contribution of all these effects to the observation. A very recent tool, developed for decoupling dynamical and structural features is the multidimensional high harmonic spectroscopy (MHHS) [150]. The concept of MHHS is based on the introduction of additional controlled parameters into the scheme, which increases the dimensionality of measurement. Importantly, the addition of this new parameter is perturbative to the HHG process.

The perturbation that we will focus on in this work is the addition of the laser field with the frequency $n \omega$ to the driving laser field with frequency $\omega$, with controlled phase $\phi$ between the two fields, $F=F_{1} \cos (\omega t)+F_{n} \cos (n \omega t+\phi)$. The new field is relatively weak in comparison to the fundamental. The perturbative nature of the extra harmonic $n \omega$ allows one to implement a 'gating' technique: the observed signal represents a modulation of the harmonic spectrum generated without the second field, with the modulation introduced by the controlled 'gate'. The mechanism of the gating process in the semi-classical picture of high harmonic generation is the perturbative change of the electron trajectories during and after tunnelling, with subsequent modulation of the recombination amplitude.

Let us consider a few important examples of this gating technique. The perturbation that we will discuss is the second harmonic ( $\mathrm{SH}$ ) of the linearly polarized laser field with the frequency $2 \omega$ and controlled phase delay $\phi$. Let the second harmonic be polarized parallel to the driving pulse. The SH breaks the symmetry between the subsequent half-cycles of the pump wave and modifies the electron trajectories differently during each half-cycle. Importantly, by modifying the action along the electron trajectory, it modifies the phase accumulated by the electron wavepacket and thus the phase of the emission (recombination) step.

As a result of different phases of the electron wavepacket at recombination, we obtain an interferometer. The two arms of the interferometer are represented with the two half-cycles of the pump laser (figure 2.3). When the scan along the two-color phase $\phi$ is performed, the harmonic spectrum is modulated in accordance with the different phases accumulated by the continuum electron during the opposite half-cycles. This breaks the symmetry between the sub- 
sequent half-cycles. Because of the symmetry breaking, weak even harmonics are generated.

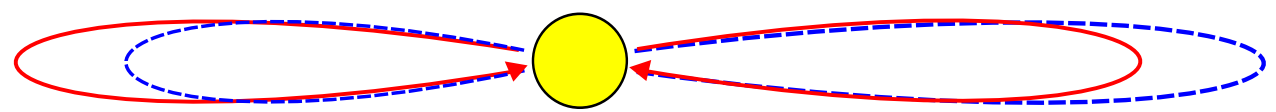

Figure 2.3: Schematic picture of the modified electron trajectories in parallel two-color field configuration: second harmonic field drives the electron away from the ion in lateral direction, modifying the recombination amplitude. Trajectory with optimal delay $\phi_{0}$ between two fields, shown in red, returns.

In the first work [151], where the gating technique with parallel SH field was implemented, the harmonic emission times were reconstructed from the experiment in argon gas. The mapping of the position of optimal two-color phases for each harmonic number on recombination times was performed within the SFA model. The results of this reconstruction from experiment were in a very good agreement with the theoretical calculation.

The same perturbative second harmonic, but with perpendicular polarization with respect to the fundamental laser field, has also been successfully implemented in multicolor gating experiments. The schematic picture of this situation is shown in figure 2.4

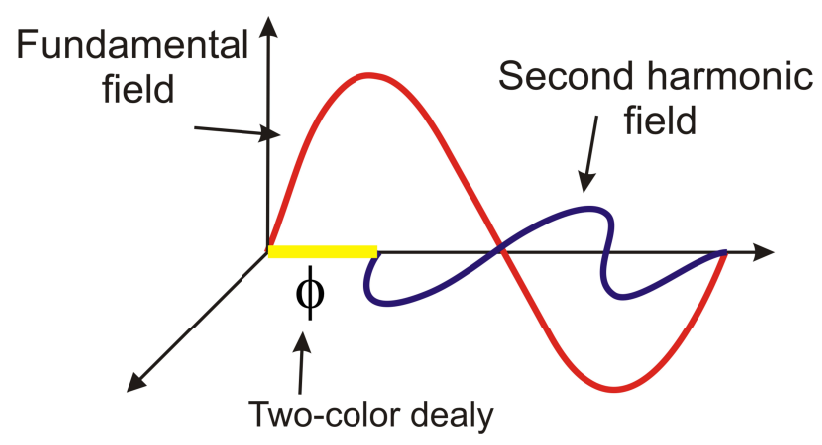

Figure 2.4: Schematic picture of the two-color field: driving laser with frequency $\omega$ shown in red, its second harmonic $2 \omega$ in blue, controlled phase delay between them $\phi$ in yellow.

On the one hand, the second harmonic field is weak enough not to disturb the 
ionization process, apart from slight modulations in ionization times as will be shown in the later chapters. On the other hand, the effect of this field on the electron propagation in the continuum is dramatic: the electron during its travel after ionization is driven in the lateral direction away from the ion, sufficiently to miss it for, thus reducing the amplitude of the recombination step (figure 2.5).

However, the phase between the two fields may have an optimal value to create such a configuration of the fields that the electron returns to the ion, for this particular harmonic. For this optimal phase, the electron trajectory leads to efficient recombination and, consequently, most efficient generation of the XUV light. If the scan across all possible delays $\phi$ is done, the two-dimensional HHG spectrum will have maximum for the optimal phase $\phi_{0}$. The position of the phase $\phi_{0}$ has universal character for atoms, because it determines the optimal field parameters for the electron return, independently of the atomic species. The situation in molecules turns out to be different. The values of the optimal delay are not determined solely by the field parameters, because the structural and the dynamical features, characteristic for molecular HHG, affect all steps of HHG and uniquely modify the spectrum.

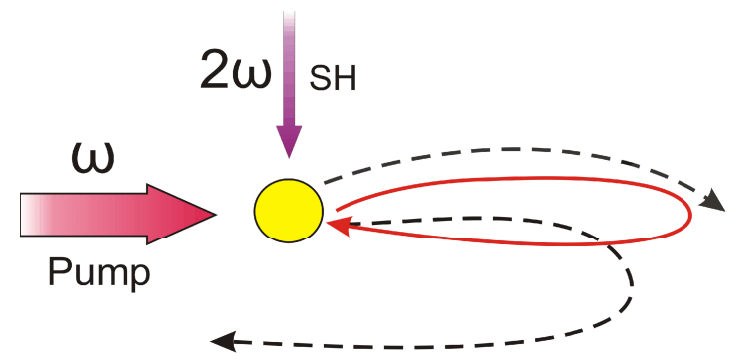

Figure 2.5: Schematic picture of the modified electron trajectories in twocolor field: second harmonic field drives the electron away from the ion in lateral direction, modifying the recombination amplitude. Trajectory with optimal delay $\phi_{0}$ between two fields, shown in red, returns.

The last point was illustrated in the experiment in $\mathrm{D}_{2}, \mathrm{~N}_{2}$ and $\mathrm{CO}_{2}$ [152], where the perpendicular two-color scheme was implemented. The $2 \mathrm{D}$ harmonic 
spectrum showed different modulation for these molecules. and its correlation with the molecular orbitals were shown.

Theoretical investigation in the atomic system ( $\mathrm{Ar}, \mathrm{He}, \mathrm{Na}$ ) and the analysis the two-color HHG experiments in the parallel field configuration was done in [153]. It was shown, that in parallel configuration the additional laser field changes not only the phase of the returning electron trajectory, but also its amplitude due to modification of the ionization step. The authors concluded that, for reliable characterisation of the emission times via the two-color harmonic generation, one needs accurate determination of the experimental intensity, which allows one to include the changes in the ionization amplitude as a function of $\phi$. This point was further developed and used to characterize the ionization dynamics in our work [154], as discussed later in this Thesis. Comparison of calculations within the SFA approach and the classical model demonstrated that classical description of HHG with the two-color fields shows significant deviation from the quantum SFA-based model. Another remarkable result of this investigation is the demonstration of the dominance of the long trajectories contribution over the short trajectories in the 2D harmonic spectrum of Ar near the cutoff region.

A significant step in the implementation of two-color harmonic generation to understanding atomic and molecular attosecond dynamics was achieved in [93]. The two-color scheme with orthogonal fields was used for the reconstruction of both ionization and recombination times in He atom. The reconstruction of the recombination times is based on the observation of the modulation of even harmonics. Even harmonics are produced as a consequence of the breaking of the symmetry by the SH field between half-cycles of the fundamental beam. When the lateral component of the electron velocity is maximal, the symmetry breaking is maximal as well, which leads to the maximum in the ratio between adjacent even and odd harmonics. This ratio is mapped onto the recombination time of each harmonic trajectory.

Extraction of the ionization times was performed with the analysis of the spectrum of odd harmonics and its modulation as a function of the two-color delay $\phi$. As described above, the amplitude of the harmonic signal is maximized, when the conditions for the electron return are optimal, or, in other words, 
when the trajectory is optimized with a certain $\phi_{0}$. Every trajectory has a well-defined ionization and recombination times, therefore the dependence of the signal strength and of the optimal gate parameters on the harmonic number encodes the ionization and recombination times. If recombination times were determined previously, ionization times can be reconstructed. Thus, the observed position of the optimal two-color phase $\phi_{0}$ is mapped onto the ionization time of each harmonic. The results of this reconstruction were in very good agreement with the semi-classical calculation of the ionization times.

Further study of the $\mathrm{CO}_{2}$ molecule in [93] showed the ability of the twocolor gating technique to distinguish the contributions from different ionization channels in molecules. If few channels play role in HHG, then the gates imposed by the SH will be (slightly) different for each channels due to the difference in ionization potentials. The gate for the HOMO normally dominates the spectrum until the two channels interfere destructively. When it happens, the differences between gates for two channels can be observed and, thus, the distinction in the ionization dynamics between channels can be detected. 


\section{Theory of High Harmonic generation in multicolor fields}

The non-relativistic interaction of light with matter, including the interaction between strong low-frequency laser fields and atomic or molecular gases, is described by the time-dependent Schrödinger equation (TDSE) and the macroscopic Maxwell equations for light propagation. We are interested in the microscopic response of the media and consider only the interaction of intense infra-red fields with individual atoms and molecules.

There are few approaches for solving the TDSE in strong laser fields. They include (for review see [155]): numerical integration of TDSE for different effective potentials, mostly within the SAE approximation; calculations based on the Floquet theorem; systematic approximation methods using the intensefield many-body S-matrix theory [67].

Here we will discuss one such approximation, which allows for both clear physical pictures and systematic quantitative analysis is the so-called quantum orbit theory of high harmonic generation [156]. The HHG process is described within the dipole approximation and the single-active electron approximation, as discussed in chapter 1.2.1. All calculations are performed in atomic units $\left(e=m_{e}=\hbar=1\right)$.

In these approximations the TDSE for one active electron is:

$$
\begin{aligned}
i \frac{\partial}{\partial t}|\Psi(\mathbf{r}, t)\rangle & =\hat{H}|\Psi(\mathbf{r}, t)\rangle \\
\hat{H} & =\hat{H}_{0}+\hat{V}_{L}(t)
\end{aligned}
$$


where $\hat{H}_{0}=\frac{\hat{\mathbf{p}}^{2}}{2}+\hat{V}_{a}(\mathbf{r})$ is the field-free atomic or molecular Hamiltonian, $\hat{V}_{L}(t)$ is the interaction between the system and the laser field.

The initial condition for this partial differential equation is $\left|\Psi\left(\mathbf{r}, t=t_{0}\right)\right\rangle=$ $\left|\Psi_{i}(\mathbf{r})\right\rangle$, where $\left|\Psi_{i}\right\rangle$ is the initial (ground) state wave function. Further we will drop the dependence on the coordinate $\mathbf{r}$ in our formulas for convenience (while remembering about it).

The exact solution of the Schrödinger equation 3.1 is:

$$
|\Psi(t)\rangle=-i \int_{t_{0}}^{t} d t^{\prime} e^{-i \int_{t^{\prime}}^{t} \hat{H}\left(t^{\prime \prime}\right) d t^{\prime \prime}} \hat{V}_{L}\left(t^{\prime}\right) e^{-i \int_{t_{0}}^{t^{\prime}} \hat{H}_{0}\left(t^{\prime \prime}\right) d t^{\prime \prime}}\left|\Psi_{i}\right\rangle+e^{-i \int_{0}^{t} \hat{H}_{0}\left(t^{\prime \prime}\right) d t^{\prime \prime}}\left|\Psi_{i}\right\rangle
$$

The first term in the wave function 3.3 represents the evolution of the system from the ground state to the final (continuum) state driven by the interaction $\hat{V}_{L}(t)$. The second term is only the field-free evolution of the ground state. Let us denote these terms schematically as $\left|\Psi_{\text {con }}\right\rangle$ and $\left|\Psi_{\text {bound }}\right\rangle$, so that $|\Psi(t)\rangle=$ $\left|\Psi_{\text {con }}\right\rangle+\left|\Psi_{\text {bound }}\right\rangle$.

We are interested in the spectrum of the emitted light as an observable. The radiation produced as a result of interaction with the laser field $\hat{V}_{L}(t)$ is proportional to the dipole moment:

$$
D(t)=\langle\Psi(t)|\hat{\mathbf{d}}| \Psi(t)\rangle
$$

In the short notation for continuum and bound parts of the wave function, the dipole moment 3.4 is:

$$
\begin{aligned}
D(t) & =\left\langle\Psi_{\text {con }}+\Psi_{\text {bound }}|\hat{\mathbf{d}}| \Psi_{\text {con }}+\Psi_{\text {bound }}\right\rangle= \\
& =\left\langle\Psi_{\text {con }}|\hat{\mathbf{d}}| \Psi_{\text {con }}\right\rangle+\left\langle\Psi_{\text {con }}|\hat{\mathbf{d}}| \Psi_{\text {bound }}\right\rangle+\left\langle\Psi_{\text {bound }}|\hat{\mathbf{d}}| \Psi_{\text {con }}\right\rangle+\left\langle\Psi_{\text {bound }}|\hat{\mathbf{d}}| \Psi_{\text {bound }}\right\rangle
\end{aligned}
$$

We neglect the first term in the 3.5, thus neglecting contribution to $D(t)$ from the free-free transitions between the continuum states, i.e. neglected harmonic generation due to free-free transitions. Of course, the free-free transitions driven by the strong laser field are included in the wavefunction. We also assume that there is no permanent dipole in the ground state, which makes 
the last term in 3.5 vanish as well.

Thus, we obtain the expression of the dipole moment in the time domain:

$$
\begin{aligned}
D(t) & =\left\langle\Psi_{\text {bound }}|\hat{\mathbf{d}}| \Psi_{\text {con }}\right\rangle+\text { c.c. }= \\
& =-i\left\langle\Psi_{i} e^{-i \int_{0}^{t} \hat{H}_{0}\left(t^{\prime \prime}\right) d t^{\prime \prime}}\left|\hat{\mathbf{d}} \int_{t_{0}}^{t} d t^{\prime} e^{-i \int_{t^{\prime}}^{t} \hat{H}\left(t^{\prime \prime}\right) d t^{\prime \prime}} \hat{V}_{L}\left(t^{\prime}\right) e^{-i \int_{t_{0}}^{t^{\prime}} \hat{H}_{0}\left(t^{\prime \prime}\right) d t^{\prime \prime}}\right| \Psi_{i}\right\rangle+\text { c.c. }
\end{aligned}
$$

The free-field evolution of the ground state is described with propagator $e^{-i \int_{t_{0}}^{t} \hat{H}_{0}\left(t^{\prime \prime}\right) d t^{\prime \prime}}$.
If we take into account that the energy of the ground state is the negative ion-
ization potential $-I_{p}$, then this propagator becomes $e^{i I_{p}\left(t-t_{0}\right)}$.

\subsection{Strong Field Approximation}

For the analytical evaluation of the exponential operator in the integral in 3.6 approximations have to be made. The full Hamiltonian consists of three parts: the kinetic energy of the electron, the binding potential of the core, and the interaction with the laser field. As a result of dealing with the intense laser fields, we cannot take perturbative approach, treating the interaction with the laser as a small parameter. Instead, we neglect completely the interaction of the departing (continuum) electron with the core. This approximation is usually referred to as the strong field approximation (SFA), first proposed by Keldysh [60] and then developed later into the Keldysh-Faisal-Reiss theory $[60,69,70]$.

SFA has a few problems with its validity as an approximation, which are described in [72] together with its justifications. The main problems are the possible dependence of the result on the gauge for the laser-matter interaction, incorrect result for ionization rates which ignore the effect of the ionic potential on tunnelling, and the specific exclusion of scattering off the ion. However, the SFA gives analytical result which allows one to build the physical intuition about the process of strong field interaction. While the SFA accuracy is only 
exponential, the corrections to the pre-exponential factor can be included in the result later, using different methods, numerical and analytical [157].

The full Hamiltonian in the strong field approximation is called the Volkov Hamiltonian and is written as follows:

$$
\hat{H}=\frac{\hat{\mathbf{p}}^{2}}{2}+\hat{V}_{L}(t)
$$

In the length gauge, the interaction term is $\hat{V}_{L}(t)=-\hat{\mathbf{d}} \mathbf{F}(\mathbf{t})$, where $\mathbf{F}(t)$ is the electric field strength of the laser and $\hat{\mathbf{d}}$ is the dipole operator acting on the system. For the electron motion in the continuum, in the laser field, the conserved value is the canonical momentum $\mathbf{p}=\mathbf{k}(\mathbf{t})-\mathbf{A}(t)$, where $\mathbf{k}(t)$ is the kinetic momentum and $\mathbf{A}(t)$ is the vector potential of the laser field:

$$
\mathbf{F}(t)=-\frac{\partial}{\partial t} \mathbf{A}(t)
$$

In the length gauge, the spatial part of the laser-driven free electron continuum wavefunction is the plane wave with the instantaneous kinetic momentum $\mathbf{k}(t)=\mathbf{p}+\mathbf{A}(t)$. The plane wave states $|\mathbf{p}+\mathbf{A}(t)\rangle$ form a complete basis in the Hilbert space for the Volkov Hamiltonian. The electron with the kinetic momentum $\left|\mathbf{p}+\mathbf{A}\left(t_{0}\right)\right\rangle$ evolves from time $t_{0}$ until time $t$ and obtains a kinetic momentum $|\mathbf{p}+\mathbf{A}(t)\rangle$. The wave function of the free electron oscillations in the laser field with Hamiltonian 3.7 is known analytically as the Volkov wave function $[72,158]$ :

$$
\Psi_{V}\left(\mathbf{r}, t, t^{\prime}\right)=\frac{1}{(2 \pi)^{3 / 2}} e^{-i S_{V}\left(\mathbf{p}, t, t^{\prime}\right)} e^{i \mathbf{k}(t) \cdot \mathbf{r}}
$$

The phase, accumulated during propagation, is called the Volkov phase:

$$
S_{V}\left(\mathbf{p}, t, t^{\prime}\right)=\frac{1}{2} \int_{t^{\prime}}^{t}(\mathbf{p}+\mathbf{A}(\tau))^{2} d \tau
$$

The full propagator of the system becomes the Volkov propagator:

$$
e^{-i \int_{t^{\prime}}^{t} \hat{H}\left(t^{\prime \prime}\right) d t^{\prime \prime}}\left|\mathbf{p}+\mathbf{A}\left(\mathbf{t}^{\prime}\right)\right\rangle=e^{-i S_{V}\left(\mathbf{p}, t, t^{\prime}\right)}|\mathbf{p}+\mathbf{A}(\mathbf{t})\rangle
$$


Let us now re-write the expression for the dipole moment 3.6:

$$
D(t)=i\left\langle\Psi_{i}\left|\hat{\mathbf{d}} \int_{t_{0}}^{t} d t^{\prime} e^{i I_{p}\left(t-t^{\prime}\right)} e^{-i S_{V}\left(\mathbf{p}, t, t^{\prime}\right)} \hat{\mathbf{d}} \mathbf{F}\left(t^{\prime}\right)\right| \Psi_{i}\right\rangle+\text { c.c. }
$$

After inserting decomposition of unity $\hat{1}=\int_{-\infty}^{\infty} d \mathbf{p}|\mathbf{p}+\mathbf{A}(t)\rangle\langle\mathbf{p}+\mathbf{A}(t)|$ in the expression 3.12 and denoting bound-continuum transitions $\mathbf{M}_{T}(t)=\left\langle\mathbf{p}+\mathbf{A}(t)|\mathbf{d}| \Psi_{i}\right\rangle$, we obtain the expression for the SFA dipole moment in a form that helps one to understand the physics behind the process:

$$
\begin{gathered}
D(t)=i \int_{t_{0}}^{t} d t^{\prime} \int d \mathbf{p} \mathbf{M}_{T}^{*}(t) e^{-i S_{S F A}\left(\mathbf{p}, t, t^{\prime}\right)} \mathbf{F}\left(t^{\prime}\right) \mathbf{M}_{T}\left(t^{\prime}\right)+c . c . \\
S_{S F A}\left(\mathbf{p}, t, t^{\prime}\right)=S_{V}\left(\mathbf{p}, t, t^{\prime}\right)-I_{p}\left(t-t^{\prime}\right)= \\
=\frac{1}{2} \int_{t^{\prime}}^{t}(\mathbf{p}+\mathbf{A}(\tau))^{2} d \tau+I_{p}\left(t-t^{\prime}\right)
\end{gathered}
$$

Let us analyse the expression 3.13. The dynamics driven by the field $\mathbf{F}(t)$ starts at different points of laser pulse $t^{\prime}$, over which the integration is performed. For each time $t^{\prime}$ the electric field $\mathbf{F}\left(t^{\prime}\right)$ initiates the transition $\mathbf{M}_{T}\left(t^{\prime}\right)$ between the ground state $\Psi_{i}$ and the continuum state $\left|\mathbf{p}+\mathbf{A}\left(t^{\prime}\right)\right\rangle$. The propagation of the electron after ionization, from time $t^{\prime}$ till time $t$, is described by 3.14 as well. Finally, the recombination is characterized by the continuum-bound transition matrix element $\mathbf{M}_{T}^{*}$ at time $t$.

The dipole moment in the frequency domain describes the spectrum of emitted light and is obtained via the Fourier transform of the dipole $D(t) 3.13$ :

$$
\begin{gathered}
\mathbf{D}(\Omega)=\frac{1}{\sqrt{2 \pi}} \int \mathbf{D}(t) e^{i \Omega t} d t= \\
=i \int d t \int_{t_{0}}^{t} d t^{\prime} \int d \mathbf{p} \mathbf{M}_{T}^{*}(t) e^{-i\left(S_{S F A}\left(\mathbf{p}, t, t^{\prime}\right)-\Omega t\right)} \mathbf{F}\left(t^{\prime}\right) \mathbf{M}_{T}\left(t^{\prime}\right)+c . c .
\end{gathered}
$$

Radiated power of the dipole 3.15 in atomic units is:

$$
P(\Omega)=\frac{2}{3 c^{3}} \Omega^{4}|\mathbf{D}(\Omega)|^{2}
$$




\subsection{Saddle point approximation}

The integrand in the expression of the dipole in frequency 3.15 and time 3.13 domains is a fast-oscillating function, because the Volkov phase 3.10 in the case of the interaction with strong low-frequency field scales as $U_{p} / \omega$ and is a very large number [53].

This fact allows one to apply the saddle point approximation [53, 157, 159] for the evaluation of the arising integrals from highly oscillating functions. Leaving out the derivation and the justification of the saddle point method itself (see e.g. $[157,159])$, we can summarize that the saddle point approximation allows one to obtain the value of a certain kind of integral along a contour on the complex plane by evaluating only the value of the integrand at the so-called saddle points, demonstrating that the integral is accumulated only around the saddle points and that the contributions from other parts of the integration contour on the complex plane are negligible. The saddle points are defined as those where the exponential part of the integrand is stationary, i.e. where its complex phase has zero derivative.

The integral, which saddle point method can be applied to, has a general form:

$$
I=\int_{C} e^{\lambda f(z)} d z
$$

where $f(z)$ is a complex function of a complex variable $z, \lambda$ is a large number, $C$ is a contour of integration on a complex plane. If we denote $z_{0}$ as the saddle point where $d f / d z=0$, then the approximated value of the above integral is:

$$
I=\left(\frac{2 \pi}{\lambda}\right)^{n / 2} \sum_{i=1}^{M} e^{\lambda f\left(z_{0}^{i}\right)} \frac{1}{\left.\operatorname{Hess}(f(z))\right|_{z=z_{0}^{i}}}
$$

where $n$ is a dimension of the approximated integral, $\left.H e s s(f(z))\right|_{z=z_{0}^{i}}$ is a Hessian matrix of the complex function $f(z)$ taken at the saddle points $z_{0}^{i}$. If the function $f(z)$ has $M>1$ saddle points, then the result of approximation is the sum of the contributions from all saddle points, which is shown in the formula 3.18 by the sum over the index $i$. 
The integral in the expression of the dipole moment, that we want to evaluate, satisfies the necessary conditions of the SPA [53] and we will implement the method. Let us write down the saddle point equations and examine them. As noted above, the saddle points $z_{0}$ are found by calculating the zeros of the derivatives of the phase in the exponent in the integral 3.18:

$$
f^{\prime}\left(z_{0}\right)=0
$$

In the expression of the dipole moment 3.15 the phase, that needs to remain stationary, is:

$$
\begin{gathered}
S\left(\mathbf{p}, t, t^{\prime}\right)=S_{S F A}\left(\mathbf{p}, t, t^{\prime}\right)-\Omega t= \\
=\frac{1}{2} \int_{t^{\prime}}^{t}(\mathbf{p}+\mathbf{A}(\tau))^{2} d \tau+I_{p}\left(t-t^{\prime}\right)-\Omega t
\end{gathered}
$$

This phase depends on the following three variables: the canonical momentum $\mathbf{p}$, the time of ionization $t^{\prime}$, and the time of emission $t$ of the photon with frequency $\Omega$. At this moment, we still did not make any assumptions on the laser field, except that it has high intensity and low frequency.

The sequential approach to the evaluation of the three-dimensional integrals in 3.15 is described in [157] and we follow it as a very convenient way of calculation. The saddle point approximation is used for each of the one-dimensional integrals, sequentially one after another, treating the integration variables of the remaining integrals as parameters. The order of integration is the ionization time $t^{\prime}$, the canonical momentum $\mathbf{p}$, and the emission time $t$.

Thus, following [157], we obtain three equations for the saddle points:

$$
\begin{aligned}
& \frac{\partial S\left(\mathbf{p}, t, t^{\prime}\right)}{\partial t^{\prime}}=0 \\
& \frac{\partial S\left(\mathbf{p}, t, t^{\prime}\right)}{\partial \mathbf{p}}=0 \\
& \frac{\partial S\left(\mathbf{p}, t, t^{\prime}\right)}{\partial t}=0 .
\end{aligned}
$$

Remembering the expression for the phase 3.20, we may write the saddle point 
equations as:

$$
\begin{gathered}
\frac{\partial S}{\partial t^{\prime}}=\frac{1}{2}\left(\mathbf{p}+\mathbf{A}\left(t^{\prime}\right)\right)^{2}+I_{p}=0 \\
\frac{\partial S}{\partial \mathbf{p}}=\int_{t^{\prime}}^{t}(\mathbf{p}+\mathbf{A}(\tau)) d \tau=0 \\
\frac{\partial S}{\partial t}=\frac{1}{2}(\mathbf{p}+\mathbf{A}(t))^{2}+I_{p}=\Omega
\end{gathered}
$$

In the cases of circular, elliptical or multicolor fields the vector potential of the excitation field can have two components in the plane perpendicular to laser propagation direction, which we will call parallel and perpendicular $\mathbf{A}(t)=$ $\mathbf{A}_{\text {par }}(t)+\mathbf{A}_{\text {per }}(t)$. The exception is the linearly polarized field with the only component $\mathbf{A}(t)$.

For the laser field with general polarization, the phase 3.20 becomes:

$$
S\left(t^{\prime}, t, p_{\text {par }}, p_{\text {per }}\right)=\int_{t^{\prime}}^{t} d t\left(p_{\text {par }}+A_{\text {par }}\right)^{2}+\int_{t^{\prime}}^{t} d t\left(p_{\text {per }}+A_{\text {per }}\right)^{2}+I_{p}\left(t-t^{\prime}\right)
$$

Consequently, the saddle point equations $3.24,3.25$ and 3.26 take the following form:

$$
\begin{gathered}
\frac{\partial S}{\partial t^{\prime}}=\frac{1}{2}\left(p_{\text {par }}+A_{\text {par }}\left(t^{\prime}\right)\right)^{2}+\frac{1}{2}\left(p_{\text {per }}+A_{\text {per }}\left(t^{\prime}\right)\right)^{2}+I_{p}=0 \\
\frac{\partial S}{\partial p_{\text {par }}}=\int_{t^{\prime}}^{t}\left(p_{\text {par }}+A_{\text {par }}(\tau)\right) d \tau=0 \\
\frac{\partial S}{\partial p_{\text {per }}}=\int_{t^{\prime}}^{t}\left(p_{\text {per }}+A_{\text {per }}(\tau)\right) d \tau=0 \\
\frac{\partial S}{\partial t}=\frac{1}{2}\left(p_{\text {par }}+A_{\text {par }}(t)\right)^{2}+\frac{1}{2}\left(p_{\text {per }}+A_{\text {per }}(t)\right)^{2}+I_{p}=\Omega
\end{gathered}
$$

The variables $t, t^{\prime}, p_{\text {per }}$ and $p_{\text {par }}$ in the expression for the phase 3.27 are all 


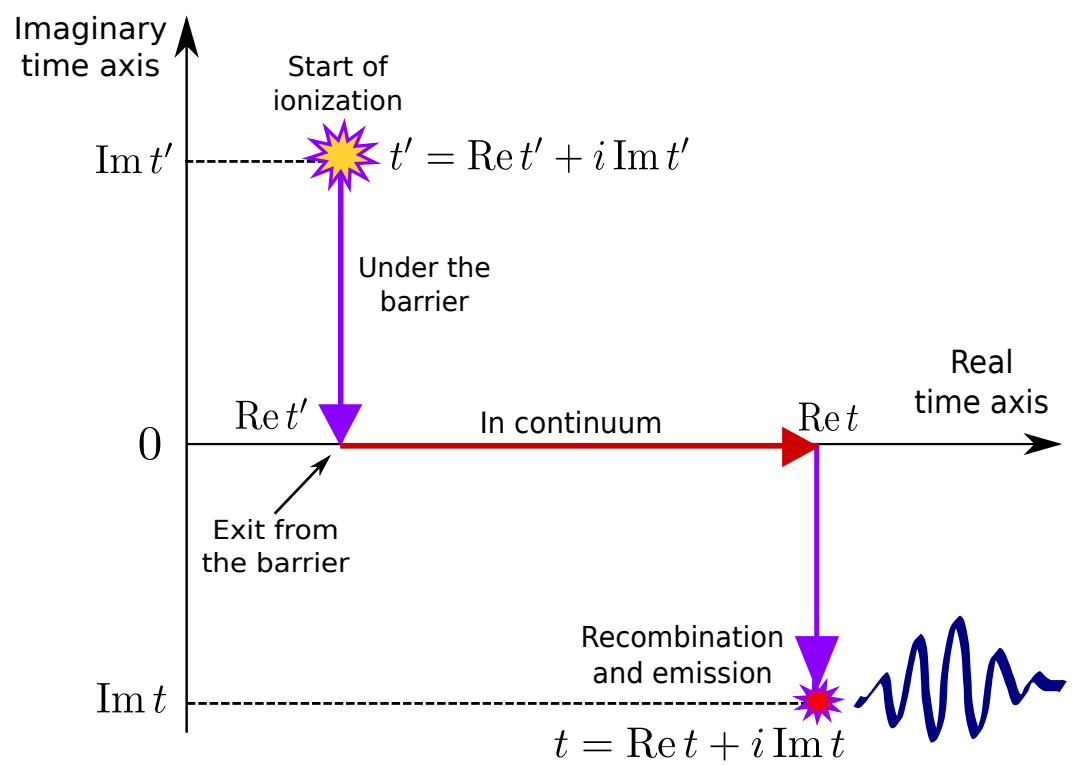

Figure 3.1: The contour of integration on the complex plane in the expression for action. Integration is performed consequently along imaginary time axis, real time axis and again imaginary time axis from complex time $t^{\prime}$ to complex time $t$

complex. The solution $t^{\prime}$ of the equation 3.24 can be found only in the complex plane. As a consequence, the solution $\mathbf{p}$ of the equation 3.25 becomes complex as well, because of the complex time $t^{\prime}$. Thus, all the parameters in the equation 3.26 are complex, which gives us complex solution for $t$.

To integrate the expression 3.27 we have to choose a contour in complex plane. The convenient contour for the calculation is presented in Figure 3.1. It shows that we first integrate only along the imaginary axis from $\operatorname{Im} t^{\prime}$ to 0 , next along the real time axis from $\operatorname{Re} t^{\prime}$ to $\operatorname{Re} t$, then again along the imaginary axis from 0 to $\operatorname{Im} t$, where the time $t^{\prime}$ is a complex ionization time and $t$ is a complex recombination time. We will denote them as $t_{i}$ and $t_{r}$ further on, respectively. Both complex ionization and recombination times are parameters and not observables. Therefore, they can be complex and we can interpret their imaginary parts within the present model. The contour of integration, shown on the figure 3.1, is preferred only in the case of calculating the phase (3.27) within the SFA. (We note that after introducing corrections to the strong field approximation due to the inclusion of the Coulomb potential, this particular 
contour may become unsuitable due to the analytic properties of the Coulomb potential $U\left(r_{L}(t)\right)$ on the complex plane of time; $r_{L}(t)$ is the electron trajectory in the laser field. Rigorous expression for Coulomb corrections to ionization times in HHG were obtained in [160].) Additionally, this contour can help us with the interpretation of the saddle point equations and lead to the recovery of the 3-step model of HHG [49], described in the chapter 1.2. The schematic illustration of the 3-step model is shown in Figure 1.1.

The first saddle point equation (3.24) describes the first step of the generation of high harmonics - ionization. It is the equation on the ionization time $t^{\prime}$ (or $t_{i}$ in the following text). The kinetic energy of the electron at the complex ionization time $t_{i}$

$$
E_{k i n}^{e}=\frac{1}{2}\left(\mathbf{p}+\mathbf{A}\left(t_{i}\right)\right)^{2}=-I_{p}
$$

is negative. This equation describes tunneling ionization of the electron under the potential barrier of an atom or a molecule with the ionization potential $I_{p}$ and with the complex kinetic momentum $\mathbf{k}\left(t_{i}\right)=\mathbf{p}+\mathbf{A}\left(t_{i}\right)$.

Equation 3.25 is an equation on the return of the electron to the parent ion. It represents the second step of the 3-step model of HHG - the propagation of the electron driven by the IR laser field. The right part of the equation is the coordinate displacement $d \mathbf{r}=\int_{t_{i}}^{t_{r}}(\mathbf{p}+\mathbf{A}(\tau)) d \tau$ between the ionization time $t_{i}$ and the recombination time $t_{r}$. The return of the electron yields $d \mathbf{r}=0$.

Equation 3.26 describes the energy conservation law upon recombination of the electron with the parent ion at a time $t$ (or $t_{r}$ in the following text) with the emission of the photon with the energy $\Omega$. It is the last step in the 3 -step model.

\subsection{Analysis of the saddle point equations: complex (quantum) trajectories.}

The direct consequence of the SFA and the SPA is the emergence of the language of the quantum electron trajectories with defined complex coordinate $\mathbf{r}$, 
complex kinetic momentum $\mathbf{p}+\mathbf{A}(t)$, and complex times of ionization $t_{i}$ and recombination $t_{r}$.

Let us consider as an example complex trajectories for the case of HHG in the linear field, for a hydrogen atom. In this example the saddle points are found numerically, solving the system of equations (3.24), (3.25), (3.26) in MATLAB, implementing a build-in fsolve algorithm. Let us take the intensity of the pump laser beam $I=10^{14} \mathrm{~W} / \mathrm{cm}^{2}$ a.u. with $800 \mathrm{~nm}$ carrier wave length. The values of saddle points $t_{i}, t_{r}$ and $\mathbf{p}$, which has only one component along the field $p$, are presented on the plots on figure 3.2. The qualitative behavior of the real parts for these saddle points are very similar to the behavior of the same variables calculated in the semi-classical model, which treats the electron motion classically and incorporates the tunnelling step 'by hand'. The calculated ionization and recombination times were shown in Figure 1.6 in the chapter on semi-classical description of high harmonic generation 1.2. The results, obtained in both approaches, are now shown in Figure 3.3.

The first noticeable difference between the results of the calculations in the semi-classical model and the SPA analysis (also referred to as quantum trajectory and/or quantum orbit analysis) of the quantum SFA is the energy of the cut off harmonic: the semi-classical approach underestimates it 3.3. It happens, because the semi-classical three-step model assumes initial conditions for the electron that are not correct: the electron appears in the continuum with zero coordinate and zero velocity, while in the SFA the tunnelling step is fully and consistently included. The electron exits the potential barrier with a displacement with respect to the origin, and in general with a non-zero velocity.

The additional displacement that the electron has to overcome in the quantum SFA description allows the electron to accumulate more energy before recombination and, as a result, have the higher cut off energy for the HHG spectrum [157].

The range of ionization times, obtained within the SFA, has a cut off as well (figure 3.3): for these laser parameters there are no ionization events happening after about 250 as after the peak of the electric field. The electron trajectories ionized before the laser peak do not return to the core and we do not consider 
them. The limited range of the ionization times is usually referred to as an ionization window, which in our example is about 250 as long. This behavior is universal but scales with the parameters of the calculation.
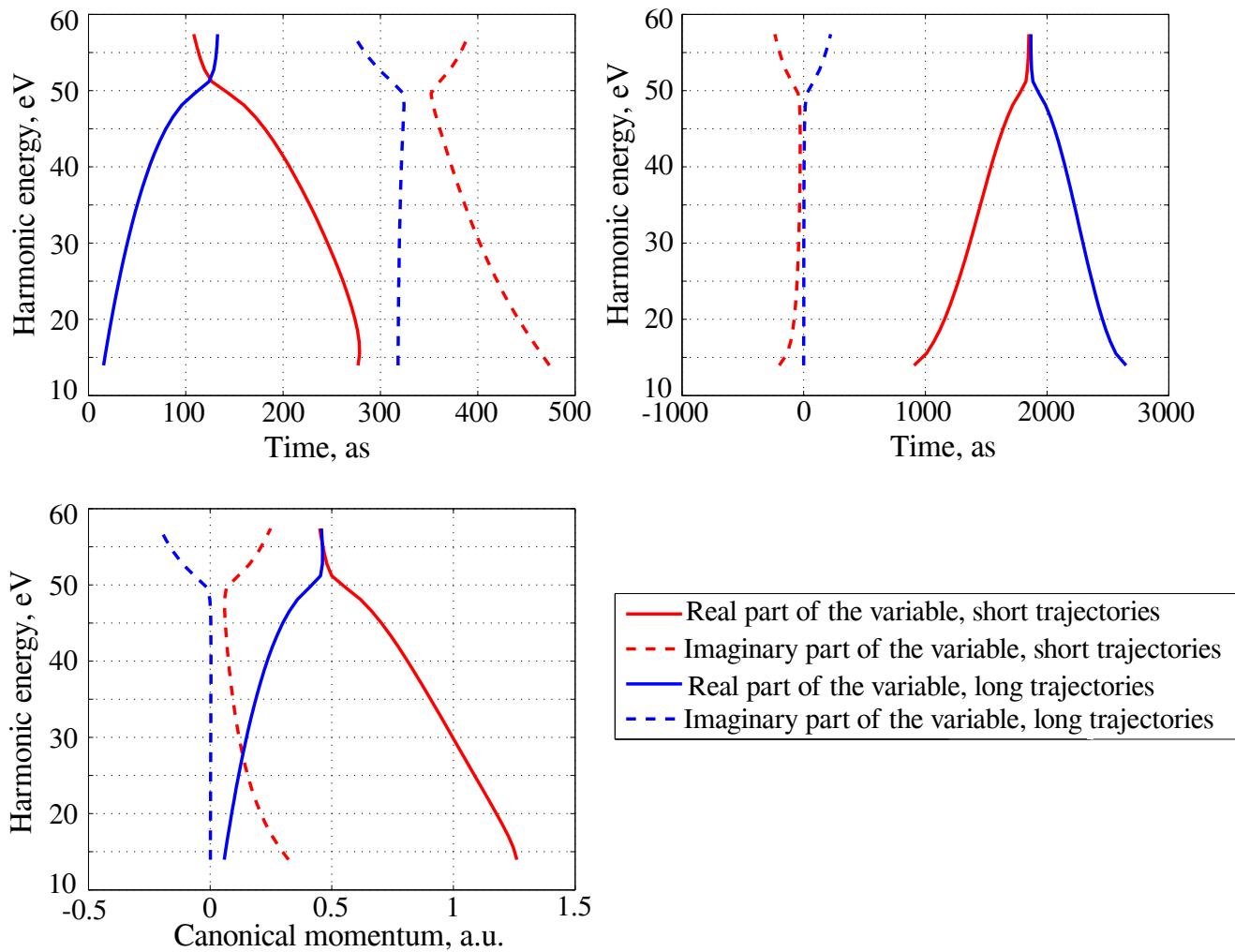

Figure 3.2: Complex saddle points solutions for ionization time, recombination time and canonical momentum, obtained in SFA model, for HHG in linear $800 \mathrm{~nm}$ field with intensity $I=10^{14} \mathrm{~W} / \mathrm{cm}^{2}$ in hydrogen. Continuous curves present real part of the variable, dotted curves - imaginary part. Red color corresponds to short trajectories, blue - long trajectories. 


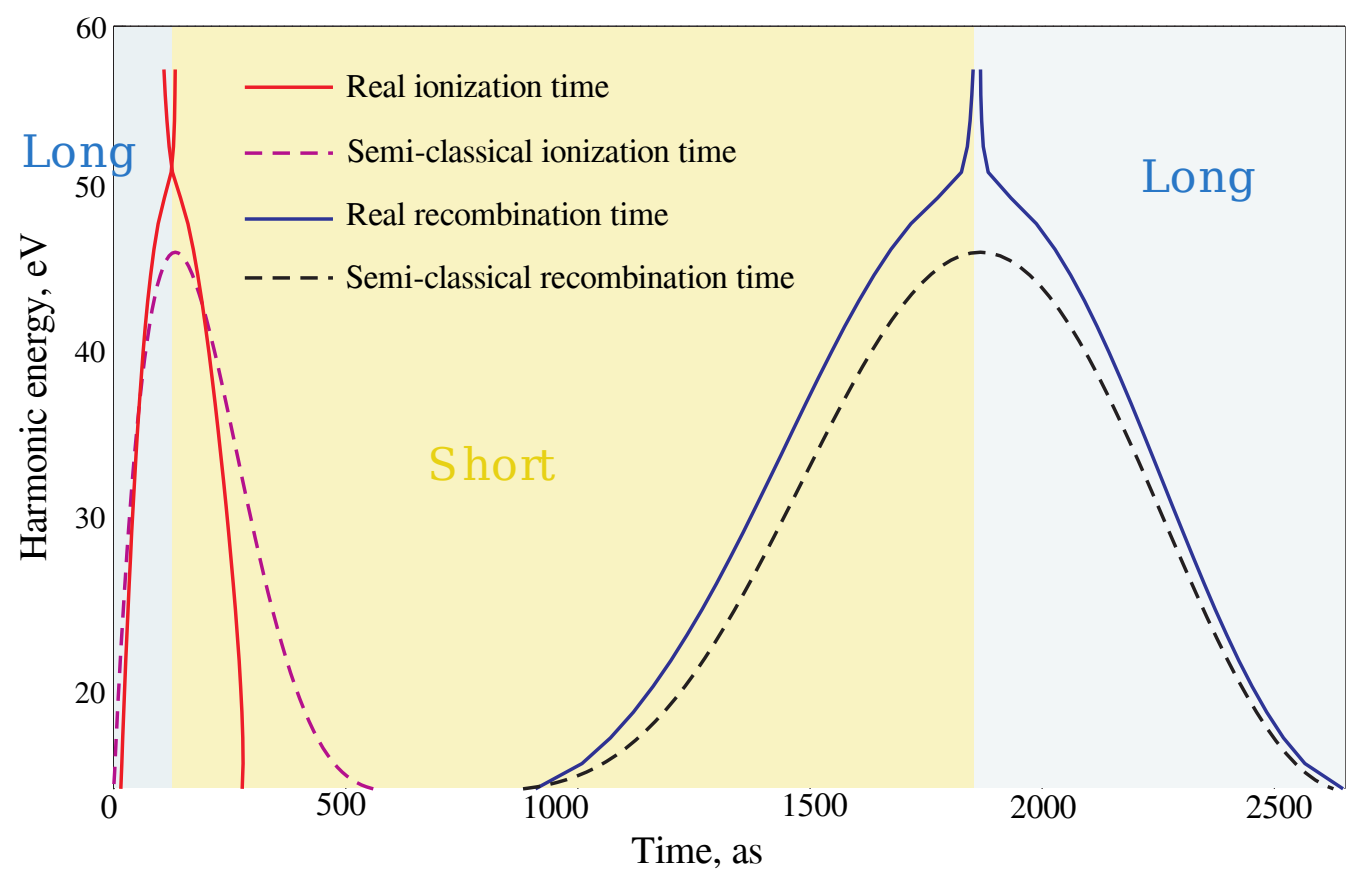

Figure 3.3: Comparison between real parts of saddle point solutions, obtained for ionization and recombination times in SFA model and semiclassical values of ionization and recombination times. Calculation is performed for for HHG in linear $800 \mathrm{~nm}$ field with intensity $I=10^{14} \mathrm{~W} / \mathrm{cm}^{2}$ in hydrogen.

Another marked property of the SFA model, described above, is the divergent behavior of both real and imaginary parts of the saddle point solutions after the cut off (figures 3.2 and3.3). It appears, when the solutions for the long and short trajectories merge in the cut off [157].

\subsubsection{Harmonic dipole in the frequency domain}

Once the solutions $t_{i}, t_{r}$, and $\mathbf{p}$ for all saddle point equations ((3.28), (3.29), (3.30)) are obtained for one half-cycle, the expression for the dipole moment can be written according to the saddle point approximation [159], [157]:

$$
\begin{gathered}
\mathbf{D}(\Omega)=\left(\frac{2 \pi}{i S_{t_{i}, t_{i}}^{\prime \prime}}\right)^{\frac{1}{2}}\left(\frac{2 \pi}{i S_{t_{r}, t_{r}}^{\prime \prime}}\right)^{\frac{1}{2}} \frac{(2 \pi)^{3 / 2}}{\sqrt{\operatorname{det}\left(i S_{\mathbf{p}, \mathbf{p}}^{\prime \prime}\right)}} \times \\
\times \mathbf{d}^{*}\left(\mathbf{p}+\mathbf{A}\left(t_{r}\right)\right) e^{-i S\left(\mathbf{p}, t_{r}, t_{i}\right)+i \Omega t_{r}} \mathbf{F}\left(t_{i}\right) \mathbf{d}\left(\mathbf{p}+\mathbf{A}\left(t_{i}\right)\right)
\end{gathered}
$$


The process of harmonic generation repeats every half-cycle for short and long trajectories. Thus, the generated dipole moment is the result of the coherent sum of the dipoles for both sets of trajectories from each half-cycle [157] of the pulse with M cycles.

The consequence of the interference between high-energy light emitted 4 times per laser cycle is the generation of discrete harmonics $N \omega$ of the laser frequency [53] $\omega$. In the system with central symmetry and for the generating light of one color, the result of this interference is a series of odd harmonics.

The dipole then is [157]:

$$
\begin{aligned}
& \mathbf{D}(N \omega)=\sum_{j=1}^{4 M}\left(\frac{2 \pi}{i S_{t_{i}^{j}, t_{i}^{j}}^{\prime \prime}}\right)^{\frac{1}{2}}\left(\frac{2 \pi}{i S_{t_{r}^{j}, t_{r}^{j}}^{\prime \prime}}\right)^{\frac{1}{2}} \frac{(2 \pi)^{3 / 2}}{\sqrt{\operatorname{det}\left(i S_{\mathbf{p}^{j}, \mathbf{p}^{j}}^{\prime \prime}\right)}} \times \\
& \times \mathbf{d}^{*}\left(\mathbf{p}^{j}+\mathbf{A}\left(t_{r}^{j}\right)\right) e^{-i S\left(\mathbf{p}^{j}, t_{r}^{j}, t_{i}^{j}\right)+i N \omega t_{r}^{j}} \mathbf{F}\left(t_{i}^{j}\right) \mathbf{d}\left(\mathbf{p}^{j}+\mathbf{A}\left(t_{i}^{j}\right)\right)
\end{aligned}
$$

If we now remember the contour of integration for the phase 3.20 on the complex plane, shown in Figure 3.1, we are able to split the expression for the full dipole 3.34 according to this contour: from time $t_{i}$ to $\operatorname{Re}\left(t_{i}\right)$, from $\operatorname{Re}\left(t_{i}\right)$ to $\operatorname{Re}\left(t_{r}\right)$ and from $\operatorname{Re}\left(t_{r}\right)$ to $t_{r}$.

Each part of the contour represents the step of the process: ionization, propagation and recombination. Let us write down their amplitudes separately.

For ionization that starts at a complex ionization time $t_{i}$ until the time of exit $\operatorname{Re}\left(t_{i}\right)$, the amplitude is:

$$
a_{\text {ion }}\left(\mathbf{p}, t_{i}\right)=\left(\frac{2 \pi}{i S_{t_{i}, t_{i}}^{\prime \prime}}\right)^{\frac{1}{2}} e^{-i S\left(\mathbf{p}, \operatorname{Re} t_{i}, t_{i}\right)} \mathbf{F}\left(t_{i}\right) \mathbf{d}\left(\mathbf{p}+\mathbf{A}\left(t_{i}\right)\right)
$$

The prefactor for the propagation amplitude is easily calculated:

$$
\frac{(2 \pi)^{3 / 2}}{\sqrt{\operatorname{det}\left(i S_{\mathbf{p}, \mathbf{p}}^{\prime \prime}\right)}}=\left(\frac{(2 \pi)}{i\left(t_{r}-t_{i}\right)}\right)^{3 / 2}
$$


It simplifies the expression for the propagation amplitude:

$$
a_{\text {prop }}\left(\mathbf{p}, t_{r}, t_{i}\right)=\left(\frac{(2 \pi)}{i\left(t_{r}-t_{i}\right)}\right)^{3 / 2} e^{-i S\left(\mathbf{p}, \operatorname{Re} t_{r}, \operatorname{Re} t_{i}\right)}
$$

The rest of what is left in the expression for the dipole is attributed to the recombination step and it is also seen from its form:

$$
a_{r e c}\left(\mathbf{p}, t_{r}\right)=\left(\frac{2 \pi}{i S_{t_{r}, t_{r}}^{\prime \prime}}\right)^{\frac{1}{2}} e^{-i S\left(\mathbf{p}, t_{r}, \operatorname{Re} t_{r}\right)} \mathbf{d}^{*}\left(\mathbf{p}+\mathbf{A}\left(t_{r}\right)\right)
$$

The expression for the full dipole now can be re-written in an almost factorized form:

$$
\mathbf{D}(N \omega)=\sum_{j=1}^{4 M} a_{i o n}\left(\mathbf{p}^{j}, t_{i}^{j}\right) a_{\text {prop }}\left(\mathbf{p}^{j}, t_{r}^{j}, t_{i}^{j}\right) a_{r e c}\left(\mathbf{p}^{j}, t_{r}^{j}\right)
$$

Factorization cannot be complete, because of the complex value of the canonical momentum $\mathbf{p}$, which stays constant during all steps and cannot be split into 3 parts. However, the formal expression of the factorized dipole 3.39 gives a powerful tool to investigate the role of each step in the process of harmonic generation, applying different methods for each step to improve the accuracy of modeling each step, and studying how it affects agreement with experiments.

\subsubsection{Harmonic dipole in the time domain}

Another way of computing the harmonic dipole, which allows one to avoid dealing with complex recombination times, is the calculation of the dipole in the time domain $\mathbf{D}\left(t_{r}\right)$ with the consequent Fourier transform, as in equation 3.15. In this case, the recombination time is treated as a parameter before the FT is performed. Hence, only 2 saddle point equations have to be solved: the equation for the ionization 3.24 and the equation for the return 3.25.

The dipole for short or long trajectory, during one half-cycle, at each recom- 
bination time, is now [157]:

$$
\begin{array}{r}
\mathbf{D}\left(t_{r}\right)=i\left(\frac{2 \pi}{i S_{t_{i}, t_{i}}^{\prime \prime}}\right)^{\frac{1}{2}} \frac{(2 \pi)^{3 / 2}}{\sqrt{\operatorname{det}\left(i S_{\mathbf{p}, \mathbf{p}}^{\prime \prime}\right)}} \times \\
\times \mathbf{d}^{*}\left(\mathbf{p}+\mathbf{A}\left(t_{r}\right)\right) e^{-i S\left(\mathbf{p}, t_{r}, t_{i}\right)} \mathbf{F}\left(t_{i}\right) \mathbf{d}\left(\mathbf{p}+\mathbf{A}\left(t_{i}\right)\right)
\end{array}
$$

The total dipole in time domain is again the sum of all events during the laser cycle, similar to 3.34 :

$$
\begin{array}{r}
\mathbf{D}\left(t_{r}\right)=i \sum_{j=1}^{4 M}\left(\frac{2 \pi}{i S_{t_{i}^{j}, t_{i}^{j}}^{\prime \prime}}\right)^{\frac{1}{2}} \frac{(2 \pi)^{3 / 2}}{\sqrt{\operatorname{det}\left(i S_{\mathbf{p}^{j}, \mathbf{p}^{j}}^{\prime \prime}\right)}} \times \\
\times \mathbf{d}^{*}\left(\mathbf{p}^{j}+\mathbf{A}\left(t_{r}\right)\right) e^{-i S\left(\mathbf{p}^{j}, t_{r}, t_{i}^{j}\right)} \mathbf{F}\left(t_{i}^{j}\right) \mathbf{d}\left(\mathbf{p}^{j}+\mathbf{A}\left(t_{i}^{j}\right)\right)
\end{array}
$$

Factorization of the dipole in time domain is also possible for each $j$ saddle point. Ionization and propagation amplitudes are the same as for the dipole in frequency domain; the only difference is that now the recombination time is a parameter and not a saddle point:

$$
\begin{gathered}
a_{i o n}^{j}\left(\mathbf{p}^{j}, t_{i}^{j}\right)=\left(\frac{2 \pi}{i S_{t_{i}^{j}, t_{i}^{j}}^{\prime \prime}}\right)^{\frac{1}{2}} e^{-i S\left(\mathbf{p}^{j}, \operatorname{Re} t_{i}^{j}, t_{i}^{j}\right)} \mathbf{F}\left(t_{i}^{j}\right) \mathbf{d}\left(\mathbf{p}^{j}+\mathbf{A}\left(t_{i}^{j}\right)\right) \\
a_{\text {prop }}^{j}\left(\mathbf{p}^{j}, t_{r}, t_{i}^{j}\right)=\left(\frac{(2 \pi)}{i\left(t_{r}-t_{i}^{j}\right)}\right)^{3 / 2} e^{-i S\left(\mathbf{p}^{j}, t_{r}, \operatorname{Re} t_{i}^{j}\right)} \\
a_{r e c}^{j}\left(\mathbf{p}^{j}, t_{r}\right)=\mathbf{d}^{*}\left(\mathbf{p}^{j}+\mathbf{A}\left(t_{r}\right)\right)
\end{gathered}
$$

Thus, factorization is very similar to the frequency domain case:

$$
\mathbf{D}\left(t_{r}\right)=\sum_{j=1}^{4 M} a_{\text {ion }}\left(\mathbf{p}^{j}, t_{i}^{j}\right) a_{\text {prop }}\left(\mathbf{p}^{j}, t_{r}, t_{i}^{j}\right) a_{r e c}\left(\mathbf{p}^{j}, t_{r}\right)
$$

\subsubsection{Including multielectron dynamics to the harmonic dipole}

In order to describe multielectron dynamics of molecules during high harmonic generation process, it has to be included in the calculation of the harmonic dipole. The ability to factorize the HHG process will allow one to study mul- 
tielectron effects on the harmonic spectrum. Following [157], we write the expression of the Hamiltonian of the multielectron system, interacting with the strong laser field:

$$
\hat{H}=\hat{T}+\hat{V}_{C}+\hat{V}_{e e}+\hat{V}_{L}
$$

where $\hat{T}$ is the electron kinetic energy operator, interaction of electrons with the ion $V_{C}$, electron-electron interaction $V_{e e}$ and interaction with the laser field $V_{L}$ :

$$
\begin{gathered}
V_{C}=-\sum_{m} \sum_{i=1}^{N} \frac{Z_{m}}{\mathbf{R}_{m}-\mathbf{r}_{i}}, \\
V_{L}=\sum_{i} \mathbf{F}(t) \mathbf{r}_{i}, \\
V_{e e}=\frac{1}{2} \sum_{i \neq j} \frac{1}{\left|\mathbf{r}_{i}-\mathbf{r}_{j}\right|}
\end{gathered}
$$

After neglecting the correlation between the electron that has left the ion and the electrons in the ion, i.e. treating this interaction in the self-consistent field approximation, the propagator between ionization and recombination is factorized into two parts: the first describes the propagation of the electron in continuum and the second accounts for the evolution of the ion in the laser field. In this approximation the difference with the single active electron model discussed above is the additional propagator of the laser-driven dynamics in the ion: the transfer of population amplitudes between the bound states of the ion after ionization. The population amplitudes $a_{m n}$ at a time $t$, that describe transitions from the state $n$ to th estate $m$ of the ion starting at the time $t_{i}$, are the solutions of the system of coupled differential equations [157]:

$$
\frac{d a_{m n}\left(t, t_{i}\right)}{d t}=(\hat{H}+\hat{V}(t)) a_{m n}\left(t, t_{i}\right)
$$

The energies of the ionic states $E_{n}$ are the eigenvalues of the stationary Hamiltonian $\hat{H}$ and the operator $V(t)$ describes the laser-driven interactions between 
the ionic states:

$$
\begin{gathered}
\hat{H}=\left(\begin{array}{cccc}
E_{1} & 0 & \cdots & 0 \\
0 & E_{2} & \cdots & 0 \\
\vdots & \vdots & \ddots & \vdots \\
0 & 0 & \cdots & E_{n}
\end{array}\right), \\
\hat{V}(t)=\left(\begin{array}{cccc}
0 & V_{12}(t) & \cdots & V_{1 n}(t) \\
V_{21}(t) & 0 & \cdots & V_{2 n}(t) \\
\vdots & \vdots & \ddots & \vdots \\
V_{n 1}(t) & V_{n 2}(t) & \cdots & 0
\end{array}\right)
\end{gathered}
$$

Following [157], we can now write the expression for the time-dependent multielectron dipole, describing the production of the ion after ionization in the state $n^{(N-1)}$ and recombination into the ionic state $m^{(N-1)}(N$ is the number of the electrons in the neutral):

$$
\begin{gathered}
\mathbf{D}^{m n}(t)=i \int_{0}^{t} d t_{i} \int d \mathbf{p d}_{m}^{*}(\mathbf{p}+\mathbf{A}(t)) a_{m n}\left(t, t_{i}\right) e^{-i S\left(\mathbf{p}, t, t_{i}\right)} \mathbf{F}\left(t_{i}\right) \mathbf{d}_{n}\left(\mathbf{p}+\mathbf{A}\left(t_{i}\right)\right) \\
\mathbf{d}(\mathbf{p}+\mathbf{A}(t))=\left\langle\mathbf{p}+\mathbf{A}(t)|\mathbf{d}| \Psi_{n}^{D}\right\rangle \\
\Psi_{n}^{D}=\left\langle n^{N-1} \mid \Psi_{g}^{N}(\mathbf{r})\right\rangle
\end{gathered}
$$

where $\Psi_{n}^{D}(\mathbf{r})$ is a Dyson orbital - an overlap between the wave function of the ground state of the neutral and the electronic wave function of the ion in the state $|n\rangle$.

As in one electron situation, the saddle point approximation makes it possible to factorize the dipole in 3.53 for each half-cycle and each channel of ionization, living the ion in the state $|n\rangle$ and recombining to the state $|m\rangle$ :

$$
\begin{gathered}
\mathbf{D}_{m n}\left(t_{r}\right)=a_{i o n}^{n}\left(\mathbf{p}, t_{i}\right) a_{\text {prop }}^{m n}\left(\mathbf{p}, t_{r}, t_{i}\right) a_{r e c}^{m}\left(\mathbf{p}, t_{r}\right) \\
a_{i o n}^{n}\left(\mathbf{p}, t_{i}\right)=\left(\frac{2 \pi}{i S_{t_{i}, t_{i}}^{\prime \prime}}\right)^{\frac{1}{2}} e^{-i S\left(\mathbf{p}, \operatorname{Re} t_{i}, t_{i}\right)} \mathbf{F}\left(t_{i}\right) \mathbf{d}_{n}\left(\mathbf{p}+\mathbf{A}\left(t_{i}\right)\right)
\end{gathered}
$$




$$
\begin{gathered}
a_{\text {prop }}^{m n}\left(\mathbf{p}, t_{r}, t_{i}\right)=\left(\frac{(2 \pi)}{i\left(t_{r}-t_{i}\right)}\right)^{3 / 2} e^{-i S\left(\mathbf{p}, t_{r}, \operatorname{Re} t_{i}\right)} a_{m n}(t) \\
a_{r e c}^{m}\left(\mathbf{p}, t_{r}\right)=\mathbf{d}_{m}^{*}\left(\mathbf{p}+\mathbf{A}\left(t_{r}\right)\right)
\end{gathered}
$$

The resulting time-dependent dipole is the sum over all half-cycles:

$$
\mathbf{D}_{m n}\left(t_{r}\right)=\sum_{j} \mathbf{D}_{m n}^{j}\left(t_{r}\right)
$$

The harmonic spectrum, produced in each channel of the process is the FT of the dipole in time domain:

$$
\mathbf{D}_{m n}(N \omega)=\int d t_{r} \mathbf{D}_{m n}\left(t_{r}\right)
$$

Finally, the resulting harmonic spectrum is the coherent sum of contributions from all channels:

$$
\mathbf{D}(N \omega)=\sum_{m, n} \mathbf{D}_{m n}(N \omega)
$$




\section{Analysis of multicolor saddle points}

In this chapter we consider a few specific examples of the application of the saddle point approximation to the calculation of the high harmonic generation spectrum. Each example has its application to the description and understanding of the experimental results, which will be demonstrated later in the Thesis. Specifically, we will show later in the Thesis how the application of multicolor fields allows one to reconstruct ionization and recombination times for each high harmonic. The presence of the second color also allows one to control and shape electron trajectories and thus control the polarization of the emitted high harmonic light, as we have shown in our paper [161]. The analysis of the saddle point trajectories therefore plays key role in understanding the processes happening during multicolor high harmonic generation.

\subsection{Saddle point approximation in the two-color perpendicular scheme of HHG}

\subsubsection{Second harmonic field as a perturbation}

As a first and very important example we are going to examine the situation, where a weak second harmonic with perpendicular polarization is added to the driving laser field. The phase $\phi$ between these waves is controlled. The driving laser electric field is:

$$
\mathbf{F}_{\|}(t)=F_{0} \cos (\omega t) \mathbf{e}_{z}
$$


where $\mathbf{F}_{0}$ is the field amplitude, $\omega$ is the laser frequency. Currently we neglect the presence of the slow field envelope, taking it into account later as a slow modulation of the amplitude $F_{0}$. The $\mathrm{SH}$ field is:

$$
\mathbf{F}_{\perp}(t)=2 \epsilon F_{0} \cos (2 \omega t+\phi) \mathbf{e}_{x}
$$

where $\epsilon$ is a small parameter. Normally it will have values not higher than $\epsilon=0.07$, which means that SH laser pulse has intensity of about $2 \%$ of the fundamental laser intensity. The coefficient 2 in front of the perturbative parameter $\epsilon$ is a consequence of our choice to use the vector potential rather than the electric field or intensity as the key quantity characterizing the laser field. We now write the two fields via their vector potentials, with the parameter $\epsilon$ determining the ratio of their amplitudes:

$$
\begin{gathered}
\mathbf{A}_{\|}(t)=-\frac{F_{0}}{\omega} \sin (\omega t) \mathbf{e}_{z}=-A_{0} \sin (\omega t) \mathbf{e}_{z} \\
\mathbf{A}_{\perp}(t)=-\epsilon \frac{F_{0}}{\omega} \sin (2 \omega t+\phi) \mathbf{e}_{x}=-\epsilon A_{0} \sin (2 \omega t+\phi) \mathbf{e}_{x}
\end{gathered}
$$

In order to obtain the harmonic spectrum, we will need to calculate the expression for the dipole Eq.(3.41). We will do it in the time domain. Knowing the exact laser field, we can write down the equations for the saddle points that enter the formula Eq.(3.41). Plugging the expressions for the parallel and perpendicular components of the laser field into 3.28, 3.29 and 3.30, we obtain the following saddle point equations:

$$
\begin{gathered}
\frac{1}{2}\left(p_{\|}-A_{0} \sin \left(\omega t_{i}\right)\right)^{2}+\frac{1}{2}\left(p_{\perp}-\epsilon A_{0} \sin \left(2 \omega t_{i}+\phi\right)\right)^{2}+I_{p}=0 \\
\int_{t_{i}}^{t}\left(p_{\|}-A_{0} \sin (\omega \tau)\right) d \tau=0 \\
\int_{t_{i}}^{t}\left(p_{\perp}-\epsilon A_{0} \sin (2 \omega \tau+\phi)\right) d \tau=0
\end{gathered}
$$

In this system of equations the recombination time $t$ is a real-valued parameter. However, all other variables that enter the system of equations are, in general, 
complex. Thus, our system consists of six equations 4.5, 4.6, 4.7 (each contains two equations for imaginary and real parts) for six variables $t_{i}^{\prime}=\operatorname{Re}\left(t_{i}\right), t_{i}^{\prime \prime}=$ $\operatorname{Im}\left(t_{i}\right), p_{\|}^{\prime}=\operatorname{Re}\left(p_{\|}\right), p_{\|}^{\prime \prime}=\operatorname{Im}\left(p_{\|}\right), p_{\perp}^{\prime}=\operatorname{Re}\left(p_{\perp}\right), p_{\perp}^{\prime \prime}=\operatorname{Im}\left(p_{\perp}\right)$.

Further, we make the substitution $k_{\|}^{\prime}=\frac{p_{\|}^{\prime}}{A_{0}}, k_{\|}^{\prime \prime}=\frac{p_{\|}^{\prime \prime}}{A_{0}}, k_{\perp}^{\prime}=\frac{p_{\perp}^{\prime}}{A_{0}}, \varphi_{i}^{\prime}=\omega t_{i}^{\prime}, \varphi_{i}^{\prime \prime}=$ $\omega t_{i}^{\prime \prime}, \varphi_{r}=\omega t_{r}$. After calculating the integral in 4.6 we obtain:

$$
\begin{gathered}
k_{\|}^{\prime}=\frac{k_{\|}^{\prime \prime}\left(\varphi_{r}-\varphi_{i}^{\prime}\right)}{\varphi_{i}^{\prime \prime}}+\frac{\sin \varphi_{i}^{\prime} \sinh \varphi_{i}^{\prime \prime}}{\varphi_{i}^{\prime \prime}} \\
k_{\|}^{\prime \prime}=\frac{\varphi_{i}^{\prime \prime} \cos \varphi_{i}^{\prime} \cosh \varphi_{i}^{\prime \prime}-\varphi_{i}^{\prime} \cos \varphi_{r}-\left(\varphi_{r}-\varphi_{i}^{\prime}\right) \sin \varphi_{i}^{\prime} \sinh \varphi_{i}^{\prime \prime}}{\varphi_{i}^{\prime \prime 2}+\left(\varphi_{r}-\varphi_{i}^{\prime}\right)^{2}}
\end{gathered}
$$

The same procedure with expression 4.7 gives us:

$$
\begin{gathered}
k_{\perp}^{\prime}=\frac{k_{\perp}^{\prime \prime}\left(\varphi_{r}-\varphi_{i}^{\prime}\right)}{\varphi_{i}^{\prime \prime}}+\frac{\epsilon}{2} \frac{\sin \left(2 \varphi_{i}^{\prime}+\phi\right) \sinh 2 \varphi_{i}^{\prime \prime}}{\varphi_{i}^{\prime \prime}} \\
k_{\perp}^{\prime \prime}=\frac{\epsilon}{2} \times \\
\times \frac{\varphi_{i}^{\prime \prime} \cos \left(2 \varphi_{i}^{\prime}+\phi\right) \cosh 2 \varphi_{i}^{\prime \prime}-\varphi_{i}^{\prime} \cos \left(2 \varphi_{r}+\phi\right)-\left(\varphi_{r}-\varphi_{i}^{\prime}\right) \sin \left(2 \varphi_{i}^{\prime}+\phi\right) \sinh 2 \varphi_{i}^{\prime \prime}}{\varphi_{i}^{\prime \prime 2}+\left(\varphi_{r}-\varphi_{i}^{\prime}\right)^{2}}
\end{gathered}
$$

Further analytical calculation is not possible. We solve the last equation and find all saddle points using the procedure similar to one described in [157].

First, we insert $k_{\|}^{\prime}, k_{\|}^{\prime \prime}, k_{\perp}^{\prime}, k_{\perp}^{\prime \prime}$ into equation 4.7 . Then, denoting $F_{1}$ and $F_{2}$ as real and imaginary parts of equation 4.7 , we construct the function:

$$
F_{1}^{2}\left(\varphi_{i}^{\prime}, \varphi_{i}^{\prime \prime}, \varphi_{r}, \phi\right)+F_{2}^{2}\left(\varphi_{i}^{\prime}, \varphi_{i}^{\prime \prime}, \varphi_{r}, \phi\right)=0
$$

Solutions to the system of saddle point equations 4.5, 4.6, 4.7 are the zeros of the surface 4.12 , which are at the same time its minima. We solve equation 4.12 numerically in MATLAB with built-in function fminsearch, obtaining all variables. 

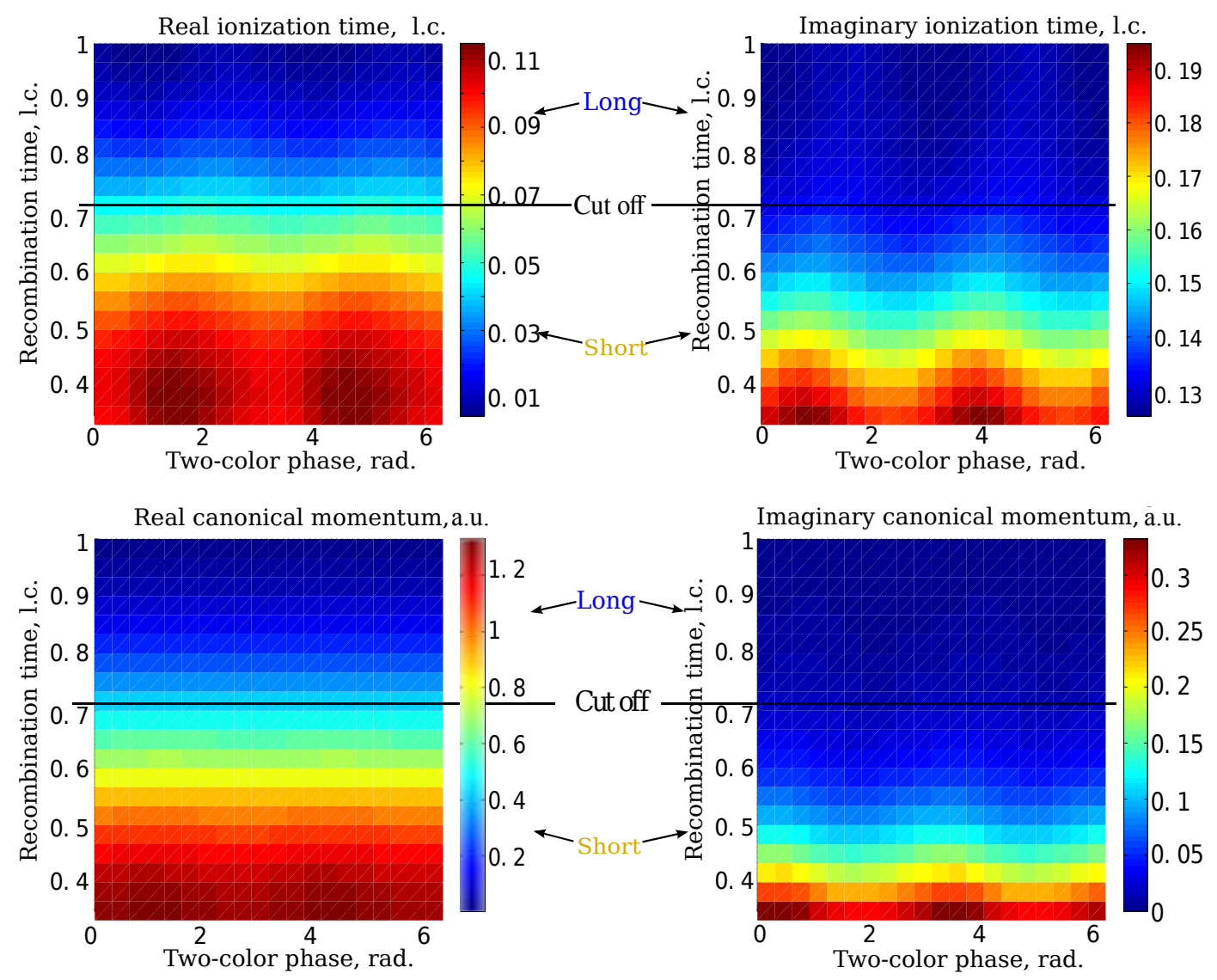

Real lateral

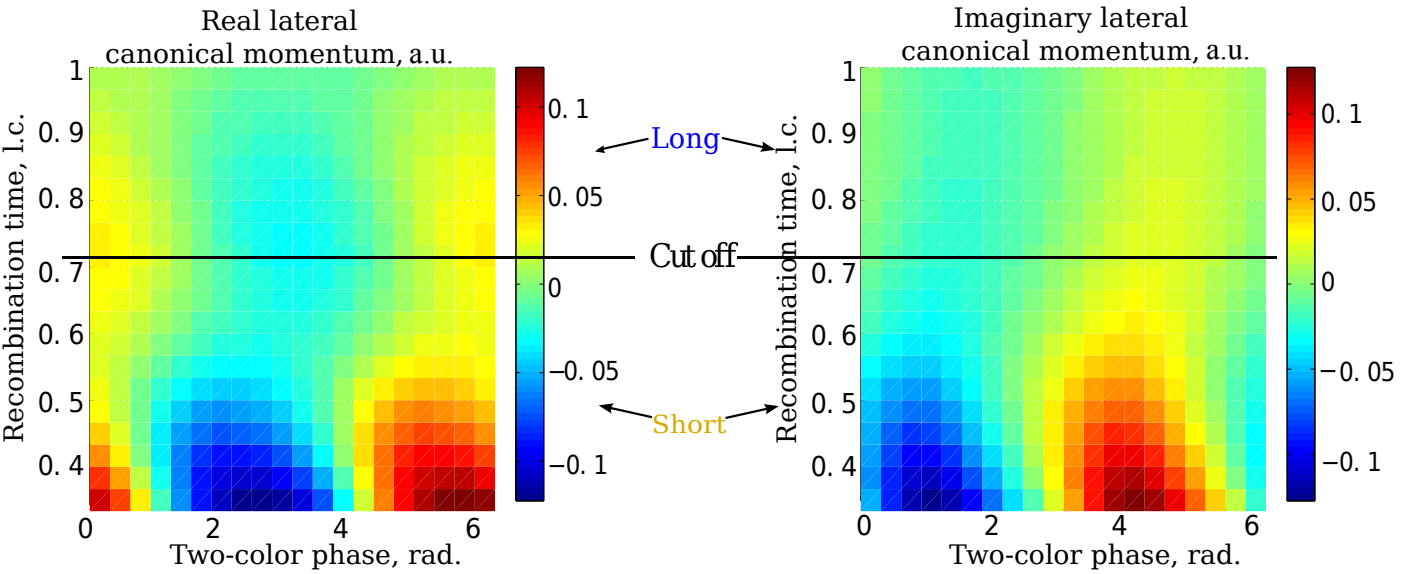

Figure 4.1: Complex saddle points solutions for ionization time and canonical momentum. Calculation for the He atom. Parameters of the driving pulse: $I_{0}=10^{14} \mathrm{~W} / \mathrm{cm}^{2}, \lambda=800 \mathrm{~nm}$, parameters of the $\mathrm{SH}$ : $\lambda_{S H}=400 \mathrm{~nm}, I_{S H}=0.02 I_{0}$. Black line shows the recombination time of the cut off trajectory in the linear field case. 
In the figure 4.1 we present the example of the results of calculating saddle points for one half cycle of the laser field for the HHG in the helium atom with the intensity of the driving laser $I=10^{14} \mathrm{~W} / \mathrm{cm}^{2}$ and its wavelength $\lambda=800$ $\mathrm{nm}$, the second harmonic field with $\lambda_{S H}=400 \mathrm{~nm}$ and $2 \%$ of the pump beam intensity. As the calculation is made in the time domain, the parameters of the electron trajectories are shown as functions of the recombination time and not the harmonic energy.
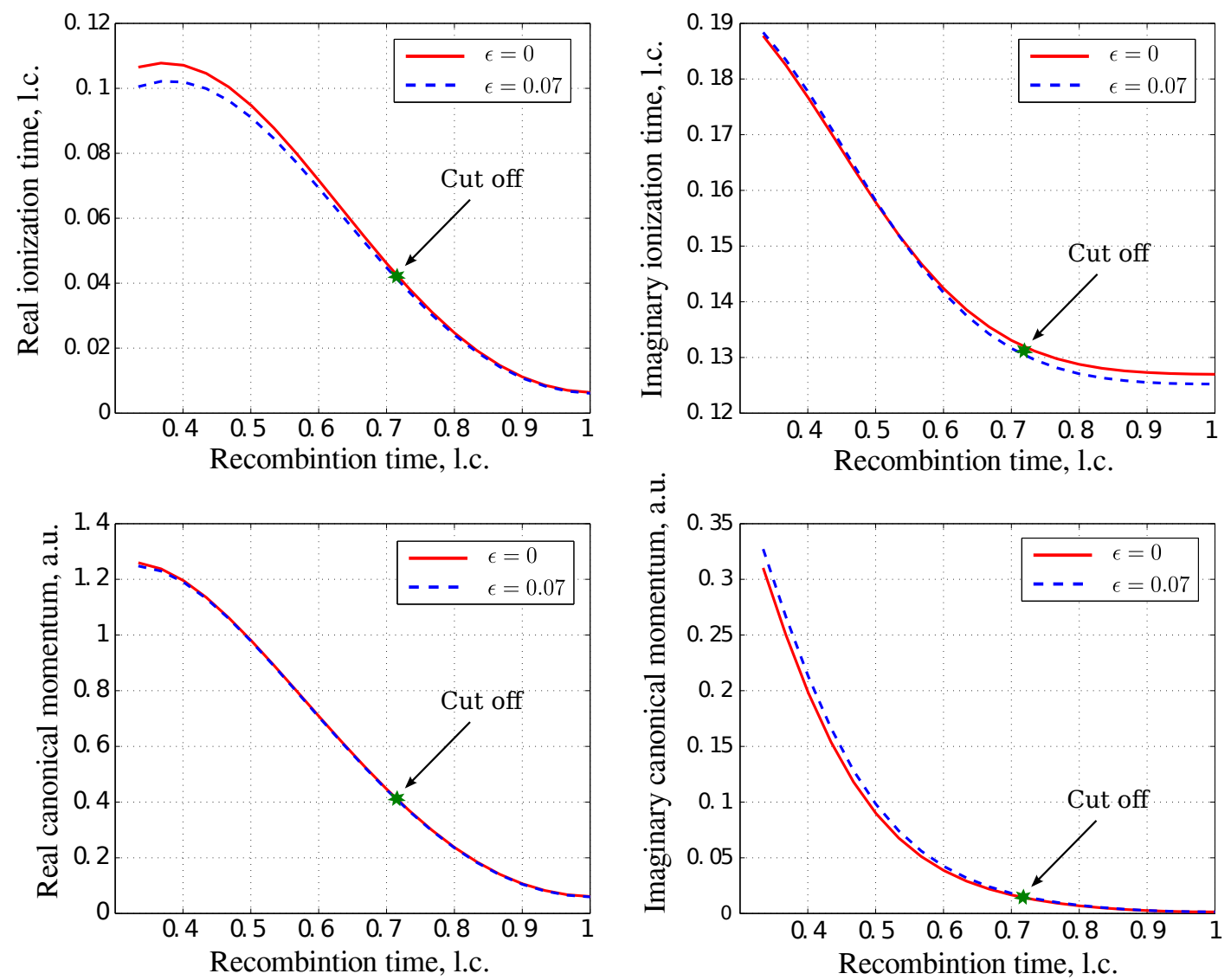

Figure 4.2: Comparison of complex saddle points solutions for ionization time and canonical momentum, obtained with (blue dotted line) and without (red line) perturbative second harmonic. Calculation for the He atom. Parameters of the driving pulse: $I_{0}=10^{14} \mathrm{~W} / \mathrm{cm}^{2}$, $\lambda=800 \mathrm{~nm}$, parameters of the $\mathrm{SH}: \lambda_{S H}=400 \mathrm{~nm}, I_{S H}=0.02 I_{0}$, two-color phase $\phi=0$.

One of the convenient aspects of this type of calculation, apart from solving 
fewer saddle point equations and dealing only with real recombination time, is that the nature of the saddle point solutions is unambiguous: for return times until $\approx 0.7 T$ of the laser cycle we are looking at the short trajectories, at $t_{r} \approx 0.7 T$ we are looking at the cut off trajectory, and then the long trajectories recombine. If we wish to find saddle points for each harmonic energy, we will get two sets of solutions for short and long trajectories, as was shown in the previous chapters.

Saddle point solutions, shown in Figure 4.1, demonstrate periodic modulation vs the two-color phase. The periodicity of the modulation is $\pi$ for the ionization time and the parallel canonical momentum and $2 \pi$ for the perpendicular component of the canonical momentum. The comparison between the saddle points obtained in the linear field only and with the SH perturbation for one two-color delay $\phi=0$ is shown in Figure 4.2.

How does the value of the perturbation parameter $\epsilon$ translates quantitatively into the modulation of the saddle points? The plots in the figure above, Fig.4.2, suggest that the values of the saddle point solutions do not change significantly. In order to further quantify the answer to this question, let us find the deviations of the saddle points obtained with the SH field present, from the linear case, expressed as a percentages of the value of the unperturbed solutions. Figure 4.3 shows the results of this simple calculation.

On the one hand, the change in the saddle point for the imaginary component of ionization time and for the real canonical momentum is within the expected few percent of the linear case solutions. On the other hand, we see significant deviation of $25 \%$ for the real ionization time and $120 \%$ for the imaginary canonical momentum. However, these large deviations are for the long trajectories, near the recombination time $t_{r}=T=2 \pi / \omega$. The unperturbed real ionization times and imaginary momenta for these trajectories are close to zero, so large percentage-wise deviation is accentuated by the small values for the unperturbed solution. Moreover, in all experiments we are going to deal with, the contributions of long trajectories are negligible due to their poor phase matching during the macroscopic propagation in the medium, especially far from the cutoff. Thus, the ratio of SH intensity and the linear field intensity of about $2 \%$ does not change significantly the ionization times and 
other parameters of the short electron trajectories, such as the momentum in the longitudinal direction.
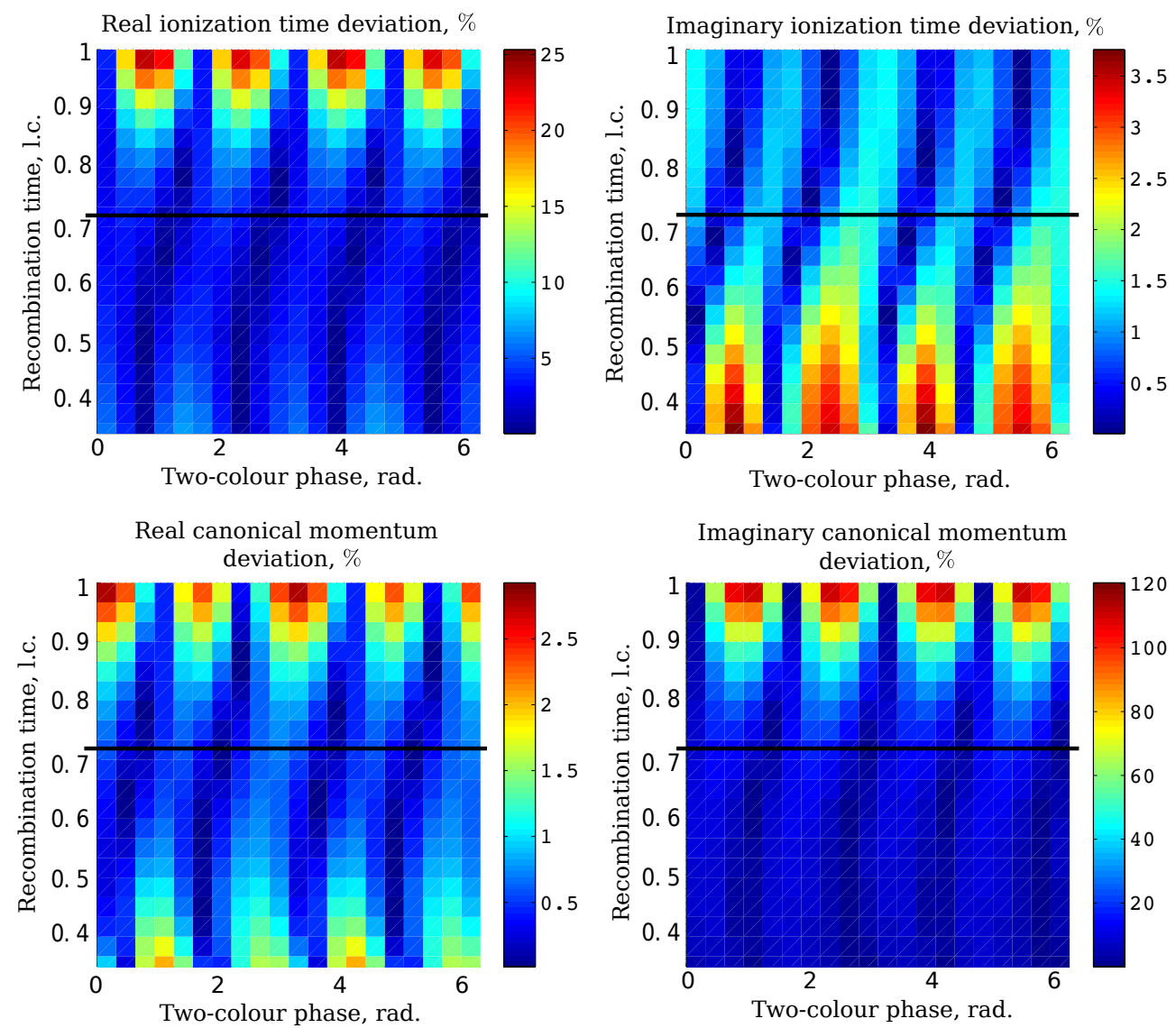

Figure 4.3: The deviation of saddle point solutions, obtained for the case with perturbative second harmonic field, from the solutions in the linear field, expressed in percentages of the value of the unperturbed solutions. Calculation for the He atom. Parameters of the driving pulse: $I_{0}=10^{14} \mathrm{~W} / \mathrm{cm}^{2}, \lambda=800 \mathrm{~nm}$, parameters of the $\mathrm{SH}$ : $\lambda_{S H}=400 \mathrm{~nm}, I_{S H}=0.02 I_{0}$. Black line shows the recombination time of the cut off trajectory in the linear field case.

After we obtained the solutions to saddle point equations 4.5, 4.6 and 4.7, it is possible to look at the amplitude modulation of the HHG signal from one half cycle. Exponential accuracy at this moment is sufficient, assuming that the pre-exponential factors associated with the geometry of the ionizing orbital and the recombination, remain the same as in the linear fundamental field, thanks to weak perturbation from the SH field. 
According to the formula 3.16, the amplitude modulation is proportional to:

$$
|\mathbf{D}(t)|^{2} \sim e^{2 \operatorname{Im} S\left(t_{i}, t_{r}, p_{p a r}, p_{p e r}, \phi\right)}
$$

We need to calculate the imaginary part of the phase 3.27 .

After the substitution $k_{\|}^{\prime}=\frac{p_{\|}^{\prime}}{A_{0}}, k_{\|}^{\prime \prime}=\frac{p_{\|}^{\prime \prime}}{A_{0}}, k_{\perp}^{\prime}=\frac{p_{\perp}^{\prime}}{A_{0}}, \varphi_{i}^{\prime}=\omega t_{i}^{\prime}, \varphi_{i}^{\prime \prime}=\omega t_{i}^{\prime \prime}, \varphi_{r}=\omega t_{r}$ the phase becomes:

$$
\begin{gathered}
S\left(\varphi_{i}, \varphi_{r}, k_{\|}, k_{\perp}, \phi\right)=\frac{A_{0}^{2}}{\omega} \int_{\varphi_{i}}^{\varphi_{r}} d \tau\left(k_{\|}+\frac{A_{\|}}{A_{0}}\right)^{2}+\frac{A_{0}^{2}}{\omega} \int_{\varphi_{i}}^{\varphi_{r}} d \tau\left(k_{\perp}+\frac{A_{\perp}}{A_{0}}\right)^{2}+ \\
+\frac{I_{p}}{\omega}\left(\varphi_{r}-\varphi_{i}\right)
\end{gathered}
$$

We now evaluate the integrals in the expression for the phase 4.14 according to the contour shown in Figure 3.1: first, along the imaginary axis from $\varphi_{i}^{\prime \prime}$ to 0 , then along the real axis from $\varphi_{i}^{\prime}$ to $\varphi_{r}$.

The first integral corresponds to the dynamics in the strong linear field:

$$
I_{1}=\int_{\varphi_{i}}^{\varphi_{r}} d \tau\left(k_{\|}+A_{\|}\right)^{2}
$$

Integration along the imaginary axis yields:

$$
\begin{aligned}
S_{1}^{\|} & =\int_{\varphi_{i}^{\prime \prime}}^{0} d \tau\left(k_{\|}+\frac{A_{\|}}{A_{0}}\right)^{2}= \\
& =\int_{\varphi_{i}^{\prime \prime}}^{0} d \tau^{\prime \prime}\left(k_{\|}^{\prime}+i k_{\|}^{\prime \prime}-\sin \left(\varphi_{i}^{\prime}+i \tau^{\prime \prime}\right)\right)^{2}= \\
& =-i \varphi_{i}^{\prime \prime}\left(k_{\|}^{\prime 2}-k_{\|}^{\prime \prime 2}+2 i k_{\|}^{\prime} k_{\|}^{\prime \prime}\right)-i \frac{\varphi_{i}^{\prime \prime}}{2}-\frac{1}{4} \sin 2 \varphi_{i}^{\prime}+\frac{1}{4} \sin 2 \varphi_{i}^{\prime} \cosh 2 \varphi_{i}^{\prime \prime}+ \\
& +\frac{1}{4} i \cos 2 \varphi_{i}^{\prime} \sinh 2 \varphi_{i}^{\prime \prime}+2\left(k_{\|}^{\prime}+i k_{\|}^{\prime \prime}\right) \cos \varphi_{i}^{\prime}\left(1-\cosh \varphi_{i}^{\prime \prime}\right)+ \\
& +2 i\left(k_{\|}^{\prime}+i k_{\|}^{\prime \prime}\right) \sin \varphi_{i}^{\prime} \sinh \varphi_{i}^{\prime \prime}
\end{aligned}
$$


Integration along the real axis yields

$$
\begin{aligned}
S_{2}^{\|} & =\int_{\varphi_{i}^{\prime}}^{\varphi_{r}} d \tau\left(k_{\|}+\frac{A_{\|}}{A_{0}}\right)^{2}= \\
& =\int_{\varphi_{i}^{\prime}}^{\varphi_{r}} d \tau^{\prime \prime}\left(k_{\|}^{\prime}+i k_{\|}^{\prime \prime}-\sin \tau^{\prime \prime}\right)^{2}= \\
& =\left(\varphi_{r}-\varphi_{i}^{\prime}\right)\left(k_{\|}^{\prime 2}-k_{\|}^{\prime \prime 2}+2 i k_{\|}^{\prime} k_{\|}^{\prime \prime}\right)+\frac{1}{2}\left(\varphi_{r}-\varphi_{i}^{\prime}\right)- \\
& -\frac{1}{4}\left(\sin \varphi_{r}-\sin \varphi_{i}^{\prime}\right)-2\left(k_{\|}^{\prime}+i k_{\|}^{\prime \prime}\right)\left(\cos \varphi_{i}^{\prime}-\cos \varphi_{r}\right)
\end{aligned}
$$

The second integral concerns only the lateral component of the trajectory:

$$
I_{2}=\int_{\varphi_{i}}^{\varphi_{r}} d \tau\left(k_{\perp}+A_{\perp}\right)^{2}
$$

Integration along the imaginary axis yields

$$
\begin{aligned}
S_{1}^{\perp} & =\int_{\varphi_{i}^{\prime \prime}}^{0} d \tau\left(k_{\perp}+\frac{A_{\perp}}{A_{0}}\right)^{2}= \\
& =\int_{\varphi_{i}^{\prime \prime}}^{0} d \tau^{\prime \prime}\left(k_{\perp}^{\prime}+i k_{\perp}^{\prime \prime}-\epsilon \sin \left(2 \varphi_{i}^{\prime}+i \tau^{\prime \prime}\right)\right)^{2}= \\
& =-i \varphi_{i}^{\prime \prime}\left(k_{\perp}^{\prime 2}-k_{\perp}^{\prime \prime 2}+2 i k_{\perp}^{\prime} k_{\perp}^{\prime \prime}\right)-i \frac{\epsilon^{2} \varphi_{i}^{\prime \prime}}{2}+\frac{\epsilon^{2}}{8} \sin \left(4 \varphi_{i}^{\prime}+2 \varphi\right)\left(1-\cosh 4 \varphi_{i}^{\prime \prime}\right)+ \\
& +i \frac{\epsilon^{2}}{8} \cos \left(4 \varphi_{i}^{\prime}+2 \varphi\right) \sinh 4 \varphi_{i}^{\prime \prime}+\epsilon\left(k_{\perp}^{\prime}+i k_{\perp}^{\prime \prime}\right) \cos \left(2 \varphi_{i}^{\prime}+\varphi\right)\left(1-\cosh 2 \varphi_{i}^{\prime}\right)+ \\
& +i \epsilon\left(k_{\perp}^{\prime}+i k_{\perp}^{\prime \prime}\right) \sin \left(2 \varphi_{i}^{\prime}+\varphi\right) \sinh 2 \varphi_{i}^{\prime \prime}
\end{aligned}
$$

Integration along the real axis yields

$$
\begin{aligned}
S_{2}^{\perp} & =\int_{\varphi_{i}^{\prime}}^{\varphi_{r}} d \tau\left(k_{\perp}+\frac{A_{\perp}}{A_{0}}\right)^{2}= \\
& =\int_{\varphi_{i}^{\prime}}^{\varphi_{r}} d \tau^{\prime \prime}\left(k_{\perp}^{\prime}+i k_{\perp}^{\prime \prime}-\epsilon \sin \left(2 \tau^{\prime}+\varphi\right)\right)^{2}= \\
& =\left(\varphi_{r}-\varphi_{i}^{\prime}\right)\left(k_{\perp}^{\prime}{ }^{2}-k_{\perp}^{\prime \prime}+2 i k_{\perp}^{\prime} k_{\perp}^{\prime \prime}\right)+\frac{\epsilon^{2}}{2}\left(\varphi_{r}-\varphi_{i}^{\prime}\right)- \\
& -\frac{\epsilon^{2}}{8}\left(\sin \left(2 \varphi_{r}+\varphi\right)-\sin \left(2 \varphi_{i}^{\prime}+\varphi\right)\right)- \\
& -\epsilon\left(k_{\perp}^{\prime}+i k_{\perp}^{\prime \prime}\right)\left(\cos \left(2 \varphi_{i}^{\prime}+\varphi\right)-\cos \left(2 \varphi_{r}+\varphi\right)\right)
\end{aligned}
$$


After the analytical calculation of the integrals above, the amplitude of the signal with exponential accuracy is given by the exponential factor:

$$
e^{2 \operatorname{Im} S\left(t_{i}, t_{r}, p_{\|}, p_{\perp}, \phi\right)}=e^{\frac{2 A_{0}^{2}}{\omega} \operatorname{Im}\left(S_{1}^{\|}+S_{2}^{\|}+S_{1}^{\perp}+S_{2}^{\perp}\right)}
$$

The calculated value of the exponent in 4.21 is presented in the figure together with its normalization, for each recombination time, to the maximum of the modulation. This normalization removes all pre-exponential factors and yields exclusively the effect of the SH perturbation on the ionization step and on the lateral displacement of the returning electron.
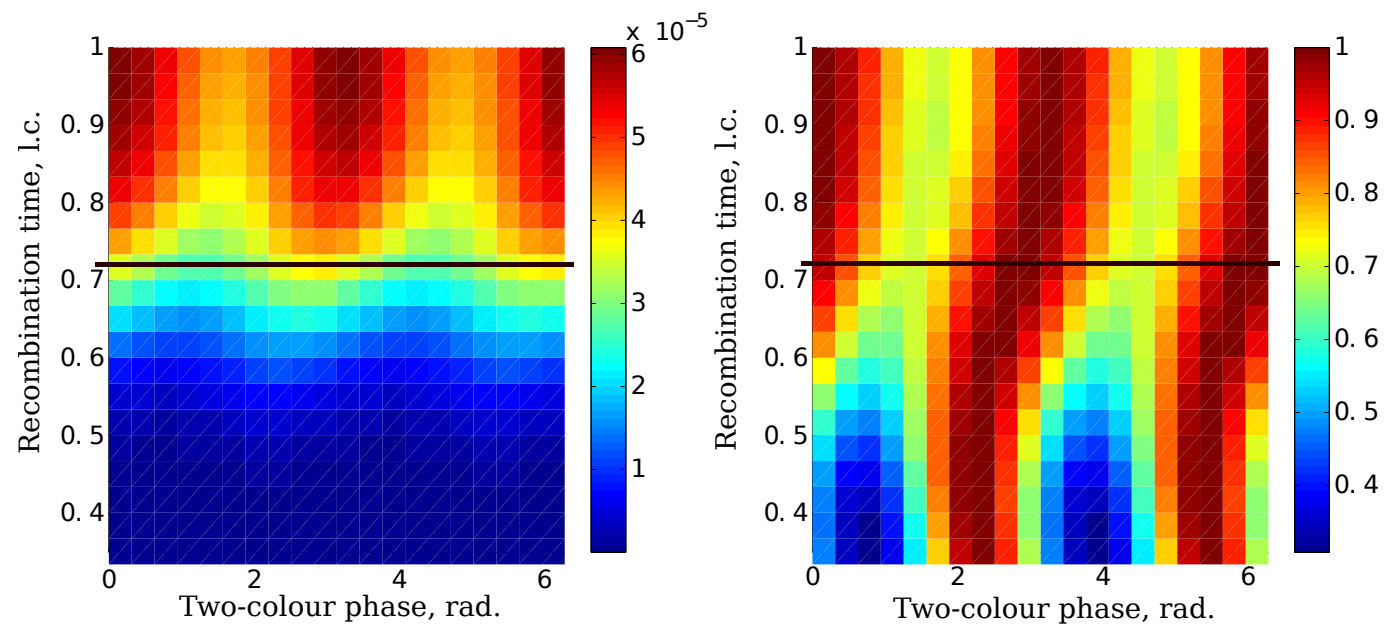

Figure 4.4: The left plot shows the calculated exponential factor 4.21. The right plot slot shows the normalization of this factor to its maximum for each recombination time. Calculations were done for the He atom. Parameters of the driving pulse: $I_{0}=10^{14} \mathrm{~W} / \mathrm{cm}^{2}$, $\lambda=800 \mathrm{~nm}$, parameters of the $\mathrm{SH}: \lambda_{S H}=400 \mathrm{~nm}, I_{S H}=0.02 I_{0}$. Black line shows the recombination time of the cut off trajectory in the linear field case.

Figure 4.4 shows that, for each recombination time, there is an optimal configuration of the two fields for re-collision. When the two-color phase between the linear and the SH fields has an optimal value, the electron does not miss the ion and radiatively recombines with the emission of a high energy photon. At this optimal phase $\phi_{\text {opt }}$, we see the maximum of the modulation on 4.4. 
It is possible to find the position of the optimal phase $\phi_{\text {opt }}$ in a few ways, that we will describe below.

Firstly, in order to check our calculations, we obtain the optimal two-color delay numerically and compare our results with it. The landscape of the twodimensional function 4.21 shows that there are no saddle points and that the function is analytical. It allows us to take slices of the surface 4.4 for each recombination time and treat them as one-dimensional functions. Each of these functions has its maximum at points $\phi_{\text {opt }}$, that we want to find. In order to find maximum or minimum of a function, we need to calculate its derivative and find where the derivative is equal to zero. We therefore obtain the expression for the derivative of the phase 4.21 and set it to zero:

$$
\begin{gathered}
-\frac{\epsilon^{2}}{8} \sinh 4 \varphi_{i}^{\prime \prime} \sin \left(4 \varphi_{i}^{\prime}+2 \phi\right)+\epsilon k_{p e r}^{\prime \prime} \sin \left(2 \varphi_{i}^{\prime}+\phi\right)\left(\cosh 2 \varphi_{i}^{\prime \prime}-1\right)+ \\
+\epsilon k_{p e r}^{\prime} \sinh 2 \varphi_{i}^{\prime \prime} \cos \left(2 \varphi_{i}^{\prime}+\phi\right)+\epsilon k_{p e r}^{\prime \prime}\left(\sin \left(2 \varphi_{i}^{\prime}+\phi\right)-\sin \left(2 \varphi_{r}^{\prime}+\phi\right)\right)=0
\end{gathered}
$$

To find the zero points $\phi_{\text {opt }}$ of the derivative 4.22 we use Newton's algorithm for solving transcendental equations, thanks to its simplicity and excellent accuracy in our case.

The second way of obtaining the optimal phase $\phi_{\text {opt }}$ is through an approximation. The optimal phase may be considered as such a delay between the fundamental field and the $\mathrm{SH}$, at which the electron is not affected by the perpendicularly polarized field right after the moment of ionization. In other words, the electron does not obtain lateral drift velocity from the $\mathrm{SH}$ at the moment of ionization. This occurs when the vector potential of SH field is zero:

$$
A_{2 \omega}\left(\varphi_{i}\right)=\epsilon A_{0} \sin \left(2 \varphi_{i}^{\prime}+\varphi_{0}\right)=0
$$

which means that optimal two-color delay $\phi_{\text {opt }}$ should be close to the points $\varphi_{0}=-2 \varphi_{i}^{\prime}+\pi$. We can expand the phase 4.21 in Taylor series near these points and find the maximum of the expansion, which corresponds to the optimal two-color delay $\phi_{o p t}$.

The third way of obtaining the position of the optimal delays $\phi_{\text {opt }}$ comes from the approximation in which the imaginary component of the momentum is 
removed from the calculation. The existence of the imaginary component of the momentum means that it is not possible, strictly speaking, to distinguish between ionization, propagation and recombination step of the HHG process. Indeed, a completely liberated electron has to have a real momentum. In the so called "photo-electron" approach, we force the momentum to be purely real. After calculating the phase 3.27 in this approach and repeating the procedure for finding the maxima of the function as the zeros of its derivative, we again obtain a set of solutions for the optimal two-color phase $\phi_{0}$.

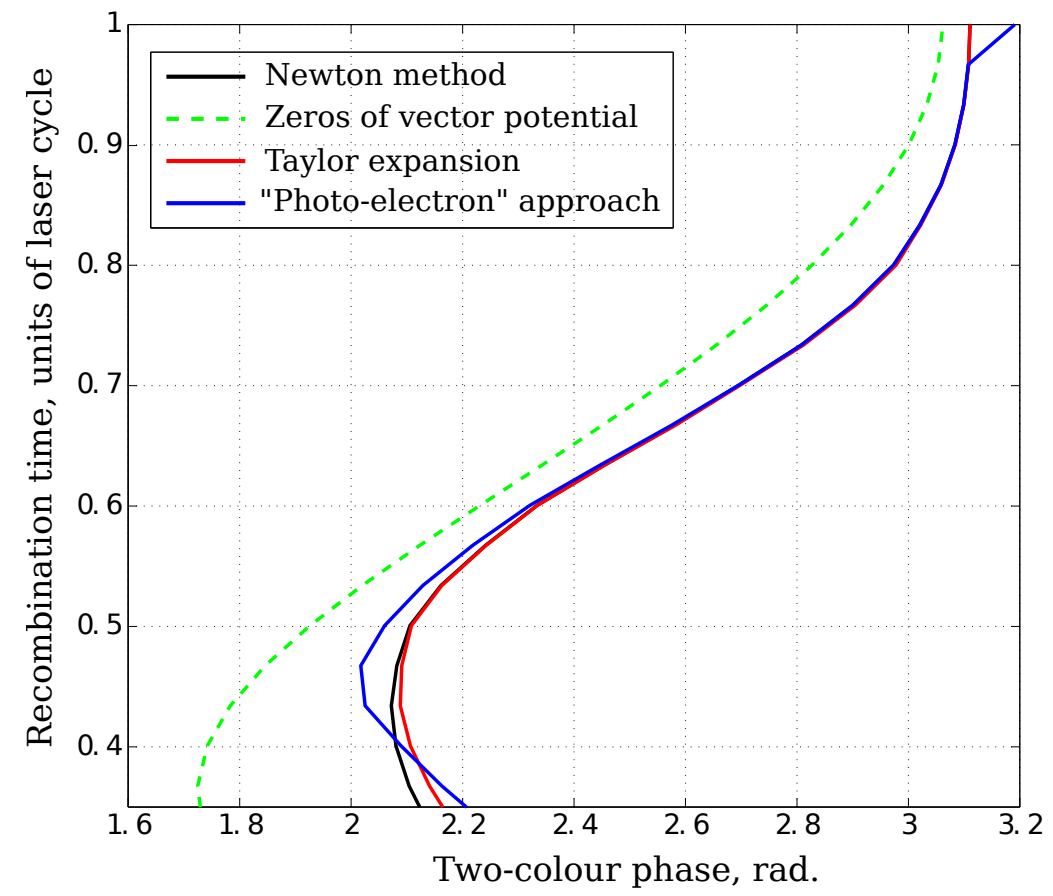

Figure 4.5: Comparison between three calculations of the optimal two-color phase $\phi_{0}$ : black curve - the zeros of the derivative of the function 4.21 , red curve - maximum points of the function 4.21, obtained after its Taylor expansion near zeros of the vector potential (green curve), blue curve - results for the maximum points of the function 4.21, obtained in 'photo-electron' approach. Calculation for the He atom. Parameters of the driving pulse: $I_{0}=10^{14} \mathrm{~W} / \mathrm{cm}^{2}, \lambda=800$ $\mathrm{nm}$, parameters of the SH: $\lambda_{S H}=400 \mathrm{~nm}, I_{S H}=0.02 I_{0}$, two-color phase $\phi=0$.

Now, we can compare solutions for the optimal two-color phase $\phi_{0}$, obtained using all the three approaches described above: the numerical calculation of the 
zeroes of the derivative, the Taylor expansion approximation, and the 'photoelectron' approximation. Figure 4.5 demonstrates that the two approximate methods (the Taylor expansion and the 'photo-electron' approach) are quite adequate, and that the only difference between the results is in the region of very short trajectories.

After obtaining the saddle point solutions, we can write the dipole moment in the form 3.41 and then make the Fourier transform:

$$
\mathbf{D}(N \omega)=\int \mathbf{D}\left(t_{r}\right) e^{i N \omega t_{r}} d t_{r}
$$

We can now use these results to compare the calculated harmonic spectrum with the experimental data presented in the paper [93]. The comparison is performed for the HHG in a He atom, generated with the intensity of the fundamental field $I_{0}=3.8 \cdot 10^{14} \mathrm{~W} / \mathrm{cm}^{2}$, the wavelength $\lambda=800 \mathrm{~nm}$, and with the perpendicularly polarized second harmonic $\lambda_{S H}=400 \mathrm{~nm}, I_{S H}=0.02 I_{0}$. The agreement with the experimental result (see the paper [93]) is excellent.
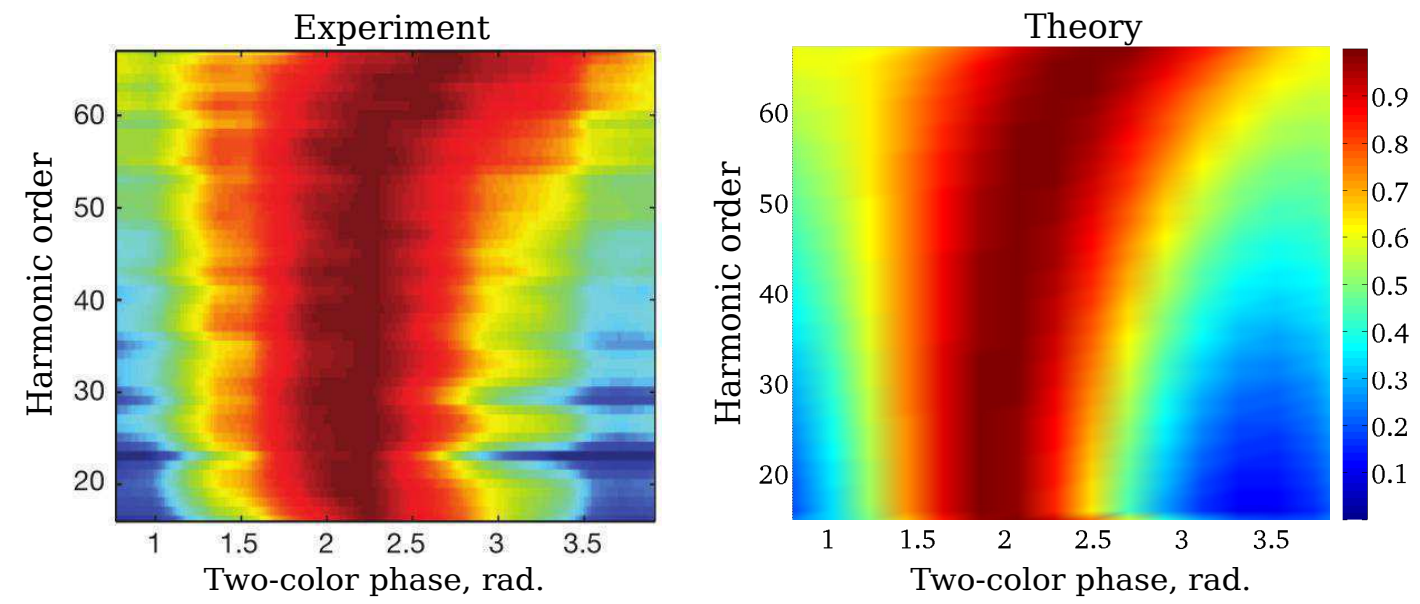

Figure 4.6: Two-dimensional HHG spectrum of He, experiment [93] (on the left) and calculation (on the right). Calculated spectrum is obtained for the parameters of the fundamental beam $I_{0}=3.8 \cdot 10^{14}$ $\mathrm{W} / \mathrm{cm}^{2}$, its wavelength $\lambda=800 \mathrm{~nm}$ and perpendicularly polarized second harmonic $\lambda_{S H}=400 \mathrm{~nm}, I_{S H}=0.02 I_{0}$. Plot shows HHG spectrum for short trajectories only. Experimental plot is adapted from [93]. 


\subsubsection{Strong second harmonic field}

In this section, we consider the influence of the second harmonic field, which is no longer weak and thus cannot be considered as a perturbation, on the quantum trajectories responsible for HHG, i.e. on the saddle point solutions.

The formulas Eqs.(4.8 - 4.20), obtained in the previous section for the saddle point solutions and for the integration of the phase 3.27, apply also to the case when $\epsilon$ is not a small parameter, because they were obtained without any assumption about its value. However, the results for saddle point solutions look dramatically different.

The first time where we notice it is when we plot the surface 4.12 for these two cases.

Figure 4.7 shows the zeros of the function 4.12, which correspond to saddle point solutions. The calculation corresponds to the return time $t_{r}=0.5$ l.c., and is done for Helium atom, $\lambda=800 \mathrm{~nm}$, and $I_{0}=10^{14} \mathrm{~W} / \mathrm{cm}^{2}$. In the case with a linear field only, we see three such points. The physically meaningful solution is the first point which corresponds to ionization time near the peak of the laser cycle centered at $t_{i} \simeq 0$, the others correspond to ionization times in the following laser half-cycles. This point has its counterpart in the twocolor field, with ionization phase $0<\varphi_{i}^{\prime}=\omega t_{i}^{\prime}<0.3$. Yet, even for small $\mathrm{SH}$ perturbation, we see the appearance of additional solutions in the area $\varphi_{i}^{\prime \prime} \approx 0.4$. 

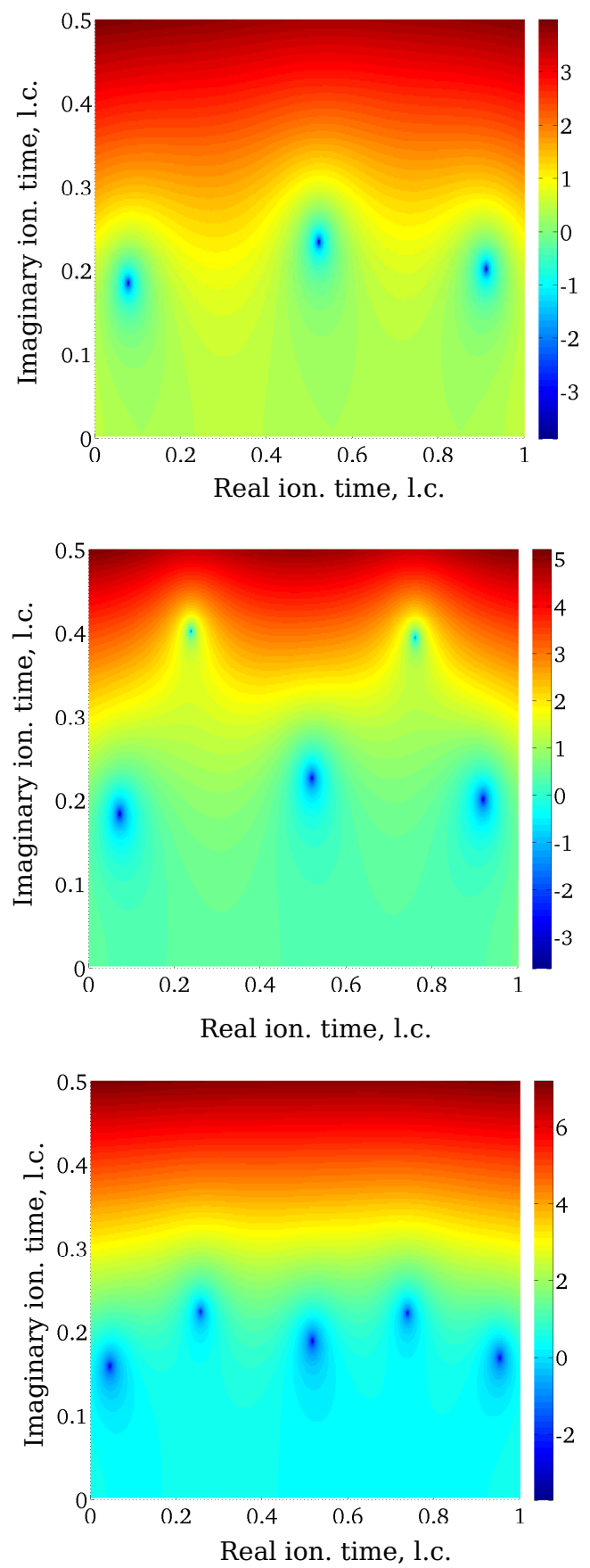

Figure 4.7: Comparison between surfaces 4.12 for different cases of the $\mathrm{SH}$ perturbation: $\epsilon=0$ (left panel), $\epsilon=0,07$ (central panel) and $\epsilon=$ 0.25 (right panel). Calculations are for the He atom. Parameters of the driving pulse: $I_{0}=10^{14} \mathrm{~W} / \mathrm{cm}^{2}, \lambda=800 \mathrm{~nm}$, parameters of the SH: $\lambda_{S H}=400 \mathrm{~nm}$, two-color phase $\phi=0$. 


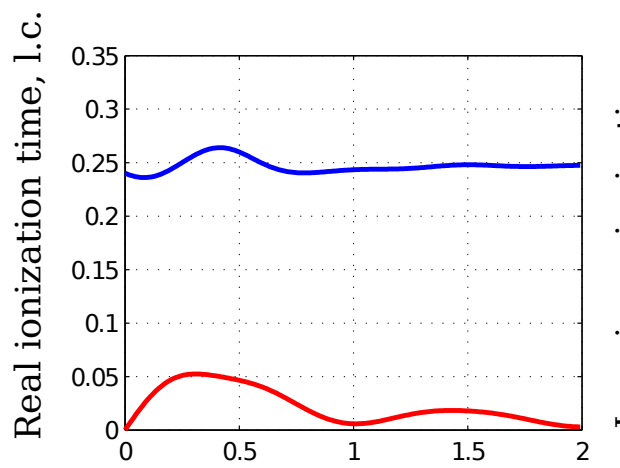

Two-color phase, rad.
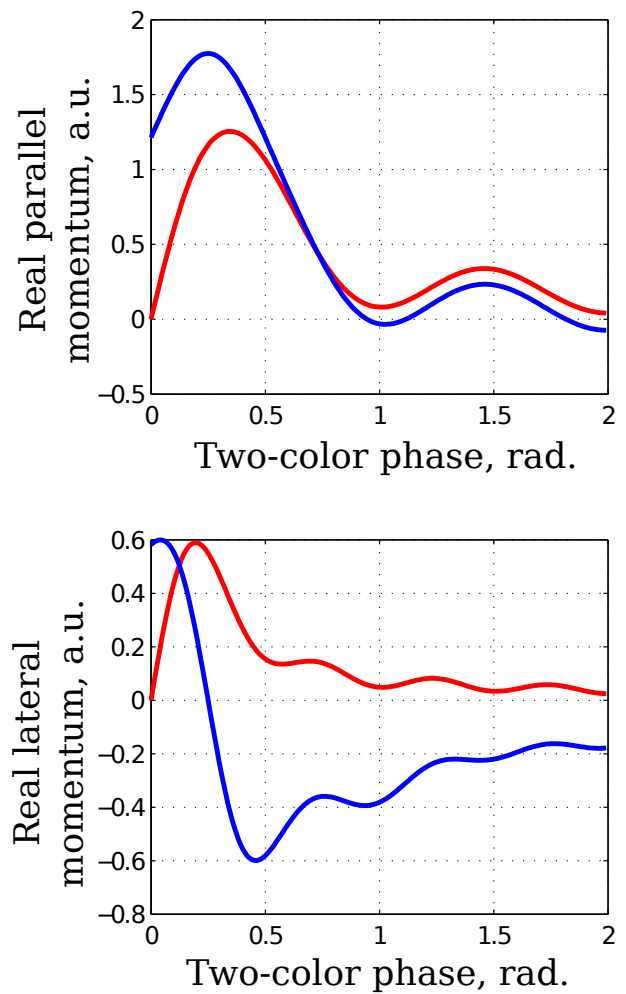

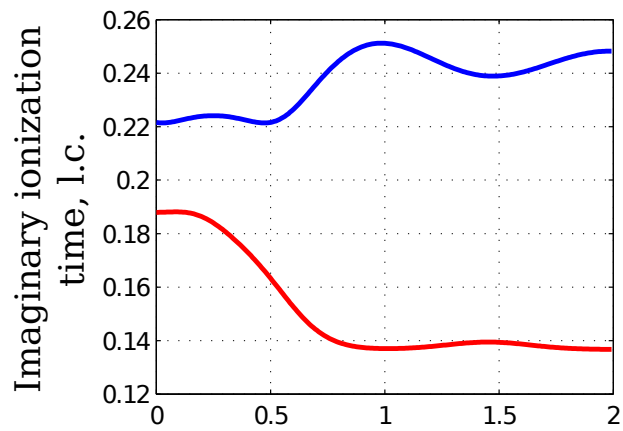

Two-color phase, rad.
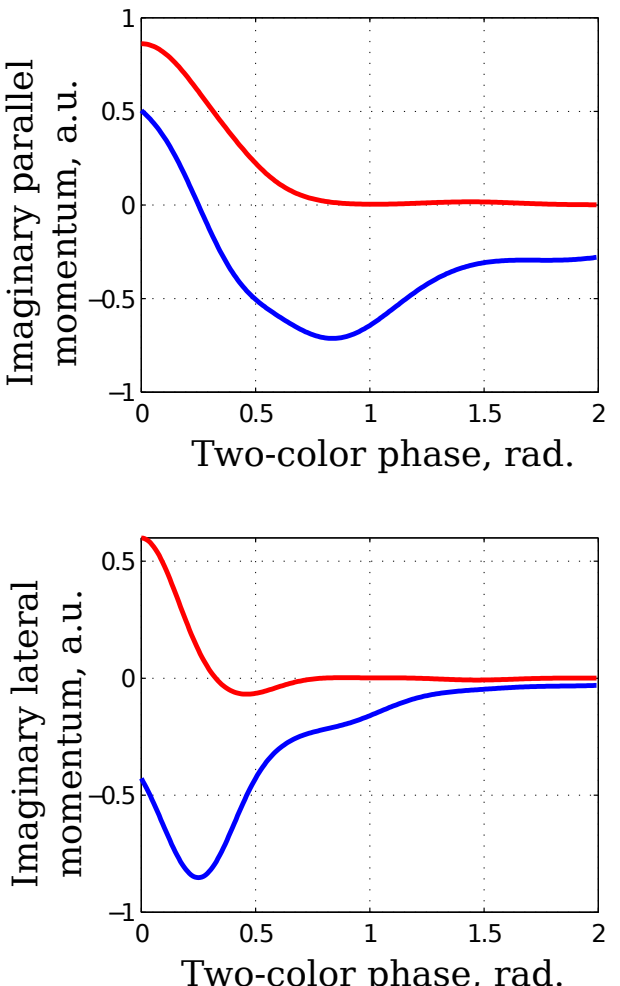

Figure 4.8: Two sets of saddle points solutions for ionization time and canonical momentum in perpendicular configuration of fundamental and strong SH field. Red curves are values of saddle points for the first minimum point on the surface 4.7 , blue curves - for the second minimum point on 4.7. Calculation for the He atom. Parameters of the driving pulse: $I_{0}=10^{14} \mathrm{~W} / \mathrm{cm}^{2}, \lambda=800 \mathrm{~nm}$, parameters of the SH: $\lambda_{S H}=400 \mathrm{~nm}, I_{S H}=0.25 I_{0}, \phi=0 \mathrm{rad}$. 
The imaginary part of ionization time determines ionization probability: the larger the imaginary ionization time, the exponentially harder it is to ionize. Therefore, the additional solution with $\varphi_{i}^{\prime \prime} \approx 0.4$ can be neglected for the perturbative $\mathrm{SH}$ field, because it does not make substantial contribution to the ionization yield. However, for the strong perpendicular $\mathrm{SH}$ field, the additional solution may become important. Interpretation of the appearance of the second set of saddle point solutions is the additional ionization burst, which happens due to the modified structure of the driving laser field.

Behavior of the two saddle points is shown in Figure 4.8 for the two-color phase $\phi=0 \mathrm{rad}$ and 4.9 for $\phi=1,52 \mathrm{rad}$. In order to take into account these new ionization events, which come from the additional saddle point solutions, one needs to add coherently the harmonic spectra produced by both sets of trajectories. The example of the calculated spectrum for the two sets of the saddle points is shown in Figure 4.10. 

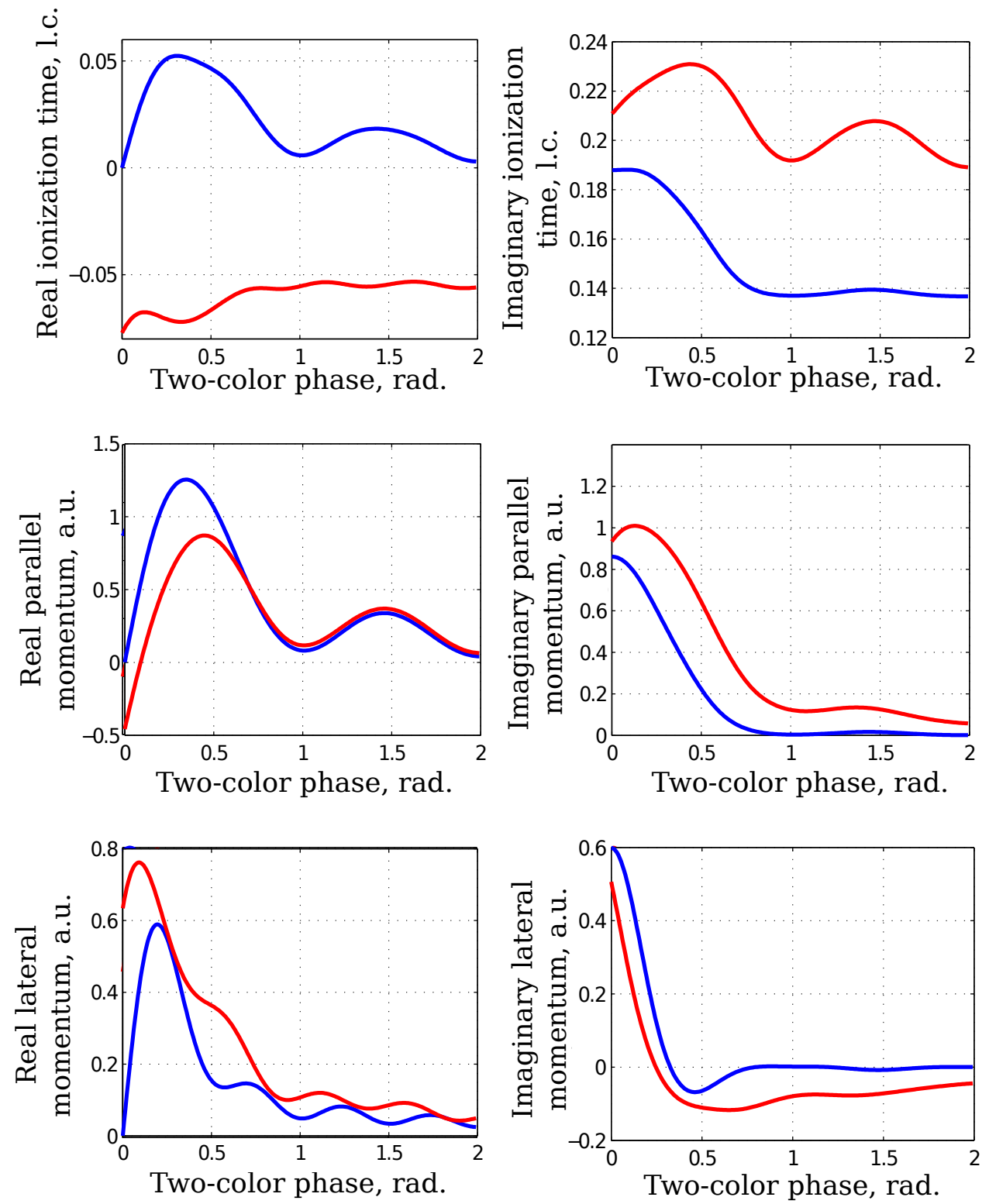

Figure 4.9: Two sets of saddle points solutions for the ionization time and the canonical momentum in the perpendicular configuration of the fundamental and the strong SH field. The red curves are values of saddle points for the first minimum point on the surface 4.7 , the blue curves are for the second minimum in Fig.4.7. Calculation is for the He atom. Parameters of the driving pulse: $I_{0}=10^{14}$ $\mathrm{W} / \mathrm{cm}^{2}, \lambda=800 \mathrm{~nm}$, parameters of the $\mathrm{SH}: \lambda_{S H}=400 \mathrm{~nm}, I_{S H}=$ $0.25 I_{0}, \phi=1,52 \mathrm{rad}$. 

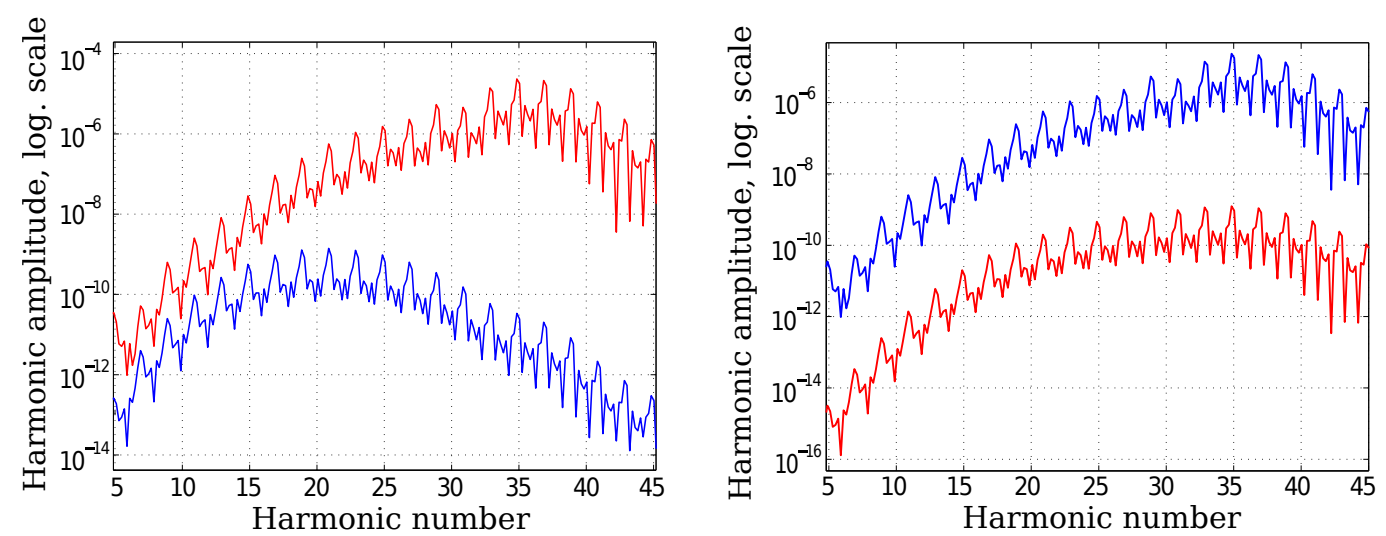

Figure 4.10: HHG spectrum calculated for two values of two-color delays $\phi=0$ rad (left plot) and $\phi=1,52 \mathrm{rad}$ (right plot). Red curves show the spectrum, calculated from the first minimum point on the surface 4.7, blue curves - from the second minimum point on 4.7 . Calculation for the He atom. Parameters of the driving pulse: $I_{0}=10^{14} \mathrm{~W} / \mathrm{cm}^{2}, \lambda=800 \mathrm{~nm}$, parameters of the $\mathrm{SH}: \lambda_{S H}=400$ $\mathrm{nm}, I_{S H}=0.25 I_{0}$.

The properties of the additional saddle points in the HHG in two-color fields with the strong SH have found its application in the attosecond pulse-shaping technique, described in the paper [161] which I have co-authored. The physical origin of the new ionization times is the possibility for the electron to ionize in the new direction, with tunnelling triggered by the strong second harmonic field. This leads to new direction of recombination and hence to a different polarization of the emitted high harmonic light associated with the new recombination event. This is one of the key aspects which allows one to shape the polarization of the harmonic pulse.

It was shown in [161] how the polarization properties of the attosecond pulses may indeed be flexibly controlled when high harmonics are generated with the mixture of the strong linear field and a SH field with the intensity of about $30-40 \%$ of the fundamental beam.

An example of such control is shown in Fig. 4.11, where a highly elliptical pulse has been generated by using the combination of the second harmonic field with $\lambda=400 \mathrm{~nm}$ and intensity of $36 \%$ of fundamental intensity $I=1.25 \times 10^{14}$ $\mathrm{W} / \mathrm{cm}^{2}$, and using the $\mathrm{CO}_{2}$ molecule as a target. The calculation included 
the dynamics induced in the molecular ion by the driving laser fields between ionization and recombination.
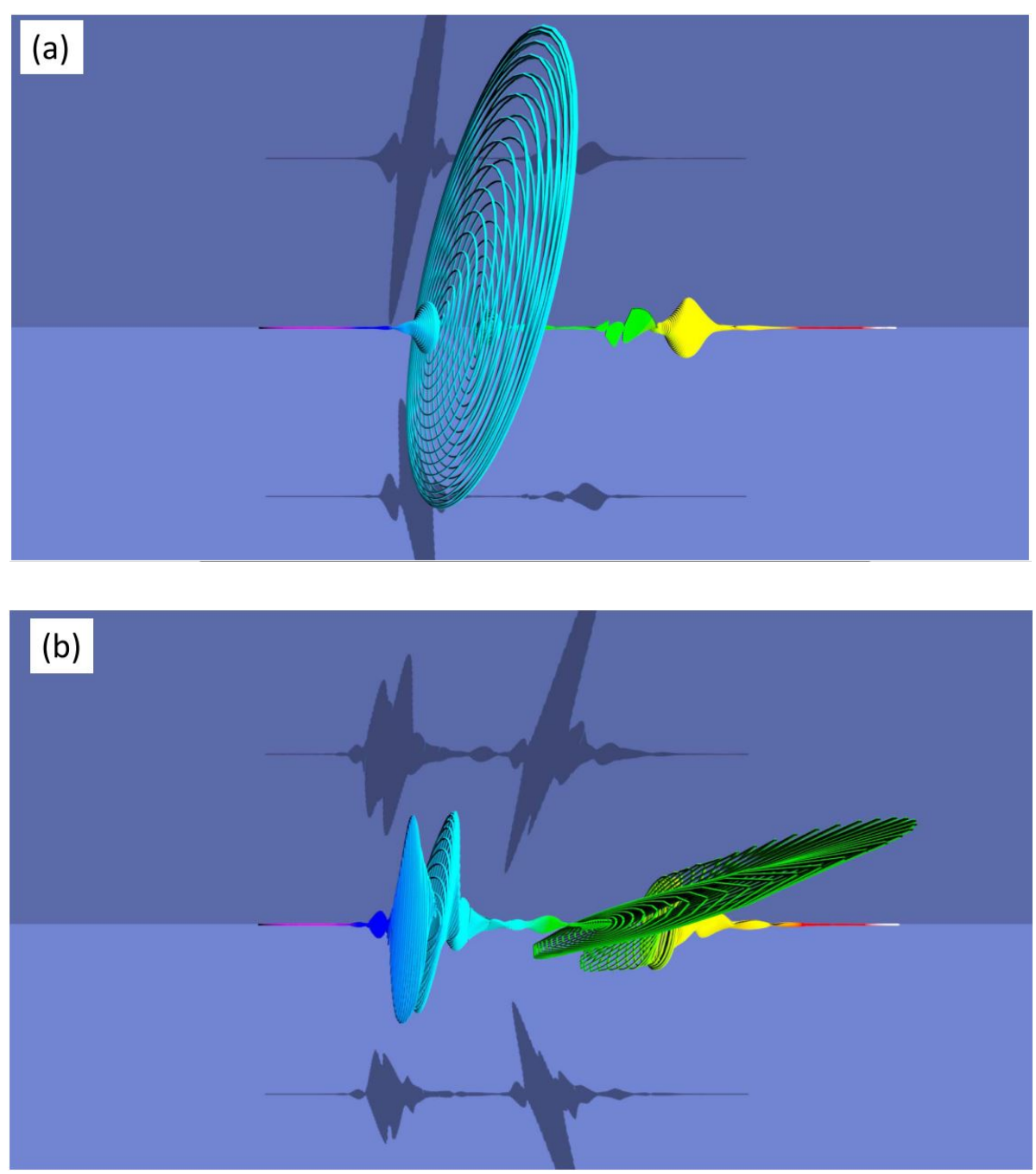

Figure 4.11: Figure from [161] of attosecond pulses, obtained using described pulse shaping technique. 


\subsection{Saddle points for the parallel configuration of the two-color field}

Analysis of the saddle point solutions in the case when the $\mathrm{SH}$ field plays a role of a perturbation with its polarization parallel to that of the fundamental field is very similar to the previously examined case of the perpendicular field configuration.

The electric field consists of two terms, the fundamental field and its second harmonic:

$$
\mathbf{F}(t)=F_{0} \cos (\omega t) \mathbf{e}_{z}+2 \epsilon F_{0} \cos (2 \omega t+\phi) \mathbf{e}_{z}
$$

where $\mathbf{F}_{0}$ is the amplitude of the electric field, $\omega$ is the frequency of the laser, $\epsilon$ is a small parameter. As before, we assume long pulses so that the envelope is absent.

We will now analyze the sub-cycle dynamics for one half-cycle of the fundamental field only. The vector potential is:

$$
\mathbf{A}(t)=-A_{0} \sin (\omega t) \mathbf{e}_{z}-\epsilon A_{0} \sin (2 \omega t+\phi) \mathbf{e}_{z}
$$

Instead of three saddle point equations, we now obtain only two, because the equation for the lateral electron motion is now absent (or, rather, simply states $\left.p_{\perp}=0\right)$ :

$$
\begin{gathered}
\frac{1}{2}\left(p-A_{0} \sin \left(\omega t_{i}\right)\right)^{2}+\frac{1}{2}\left(p-\epsilon A_{0} \sin \left(2 \omega t_{i}+\phi\right)\right)^{2}+I_{p}=0 \\
\int_{t_{i}}^{t}\left(p-A_{0} \sin (\omega \tau)-\epsilon A_{0} \sin (2 \omega t+\phi)\right) d \tau=0
\end{gathered}
$$

Thus, we need to solve four equations for four variables $t_{i}^{\prime}=\operatorname{Re}\left(t_{i}\right), t_{i}^{\prime \prime}=$ $\operatorname{Im}\left(t_{i}\right), p^{\prime}=\operatorname{Re}(p), p^{\prime \prime}=\operatorname{Im}(p)$, because each of the equations 4.27 and 4.28 may be split into the equations for real and the imaginary parts.

After the substitution $k^{\prime}=\frac{p^{\prime}}{A_{0}}, k^{\prime \prime}=\frac{p^{\prime \prime}}{A_{0}}, \varphi_{i}^{\prime}=\omega t_{i}^{\prime}, \varphi_{i}^{\prime \prime}=\omega t_{i}^{\prime \prime}, \varphi_{r}=\omega t_{r}$ from the 
equation 4.28 we obtain:

$$
\begin{gathered}
k^{\prime}=\frac{k^{\prime \prime}\left(\varphi_{r}-\varphi_{i}^{\prime}\right)}{\varphi_{i}^{\prime \prime}}+\frac{\sin \varphi_{i}^{\prime} \sinh \varphi_{i}^{\prime \prime}}{\varphi_{i}^{\prime \prime}}+\frac{\epsilon}{2} \frac{\sin \left(2 \varphi_{i}^{\prime}+\phi\right) \sinh 2 \varphi_{i}^{\prime \prime}}{\varphi_{i}^{\prime \prime}} \\
k^{\prime \prime}=\frac{\varphi_{i}^{\prime \prime} \cos \varphi_{i}^{\prime} \cosh \varphi_{i}^{\prime \prime}-\varphi_{i}^{\prime} \cos \varphi_{r}-\left(\varphi_{r}-\varphi_{i}^{\prime}\right) \sin \varphi_{i}^{\prime} \sinh \varphi_{i}^{\prime \prime}}{\varphi_{i}^{\prime \prime 2}+\left(\varphi_{r}-\varphi_{i}^{\prime}\right)^{2}}+ \\
+\frac{\epsilon}{2} \frac{\varphi_{i}^{\prime \prime} \cos \left(2 \varphi_{i}^{\prime}+\phi\right) \cosh 2 \varphi_{i}^{\prime \prime}-\varphi_{i}^{\prime} \cos \left(2 \varphi_{r}+\phi\right)-\left(\varphi_{r}-\varphi_{i}^{\prime}\right) \sin \left(2 \varphi_{i}^{\prime}+\phi\right) \sinh 2 \varphi_{i}^{\prime \prime}}{\varphi_{i}^{\prime \prime}+\left(\varphi_{r}-\varphi_{i}^{\prime}\right)^{2}}
\end{gathered}
$$

We now plug the expressions for the imaginary and the real canonical momentum 4.29 and 4.30 into the saddle point equation for ionization 4.27, and then separate the real $F_{1}$ and the imaginary $F_{2}$ parts. The saddle point values for the ionization time $\varphi_{i}=\varphi_{i}^{\prime}+\varphi_{i}^{\prime \prime}$ are numerically found as the zeros of the function:

$$
F_{1}^{2}\left(\varphi_{i}^{\prime}, \varphi_{i}^{\prime \prime}, \varphi_{r}, \phi\right)+F_{2}^{2}\left(\varphi_{i}^{\prime}, \varphi_{i}^{\prime \prime}, \varphi_{r}, \phi\right)=0
$$

After the values of the real $\varphi_{i}^{\prime}$ and imaginary $\varphi_{i}^{\prime \prime}$ times are found, they are substituted in the expressions for the canonical momentum 4.29 and 4.30, giving us the full set of saddle point solutions.

The calculated saddle points for the ionization times and the canonical momentum are shown in Figure 4.12. The parameters of the calculation are the same as in the case of the perpendicularly polarized fields: He atom, the intensity of the fundamental field is $I=10^{14} \mathrm{~W} / \mathrm{cm}^{2}$ and its wavelength $\lambda=800$ $\mathrm{nm}$, the second harmonic field with $\lambda_{S H}=400 \mathrm{~nm}$ and $2 \%$ of the pump beam intensity. The black line indicates the recombination time of the cut off trajectory in the one-color case. The periodicity of the modulation is $2 \pi$, in contrast to the perpendicular fields case $(\pi)$.

At first glance, figure 4.12 shows that the modulation of saddle point solutions due to the presence of the $\mathrm{SH}$ is very small. The comparison between the saddle points for the fundamental one-color field and for its combination with the perturbative $\mathrm{SH}$ for one value of the two-color delay $\phi=0$ is shown on the figure 4.13. 

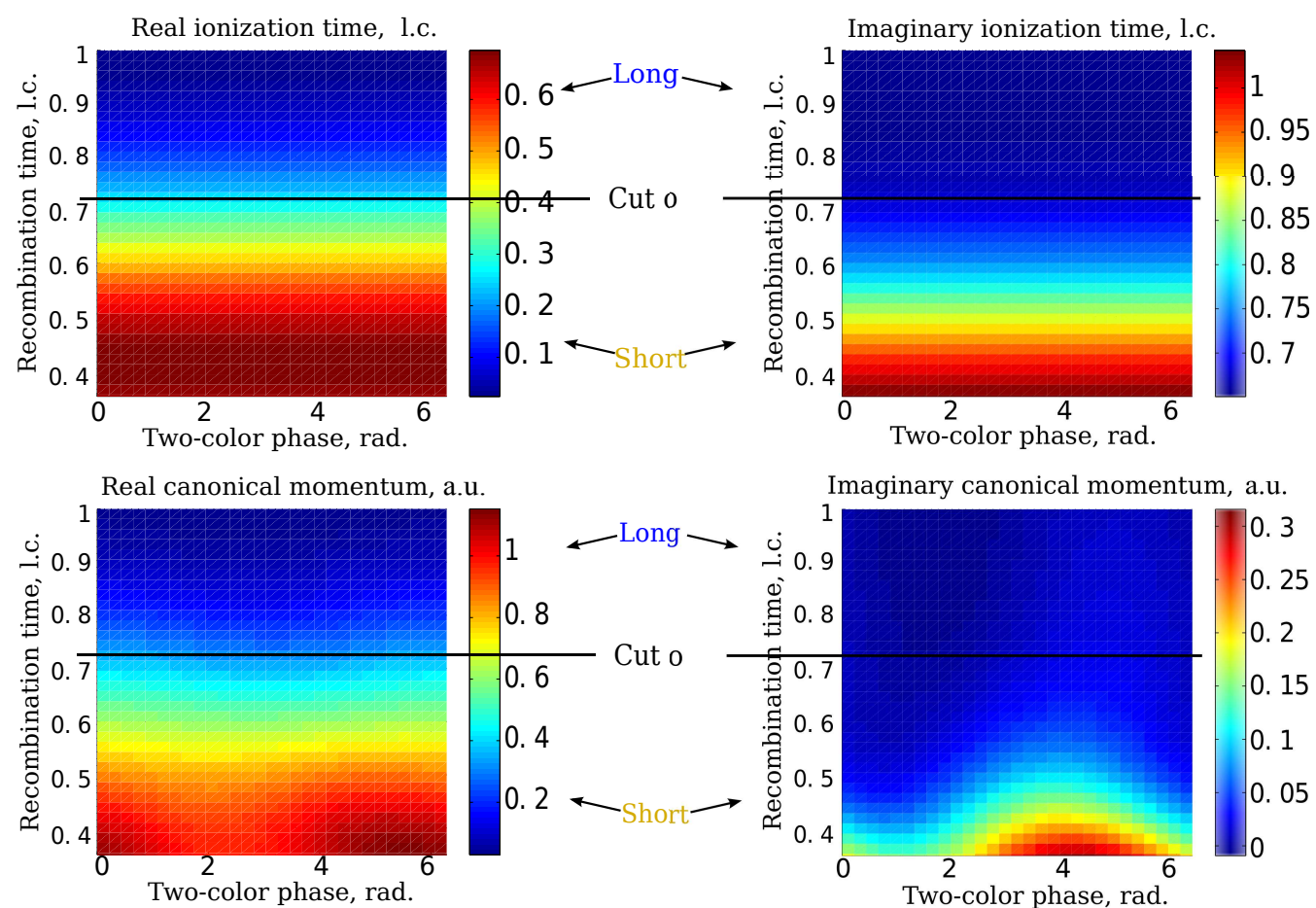

Figure 4.12: Complex saddle point solutions for the ionization time and the canonical momentum in parallel configuration of the fundamental and the SH field. Calculation are for the He atom. Parameters of the driving pulse: $I_{0}=10^{14} \mathrm{~W} / \mathrm{cm}^{2}, \lambda=800 \mathrm{~nm}$, parameters of the SH: $\lambda_{S H}=400 \mathrm{~nm}, I_{S H}=0.02 I_{0}$. Black line shows the recombination time for the cut-off trajectory in the one-color case. 

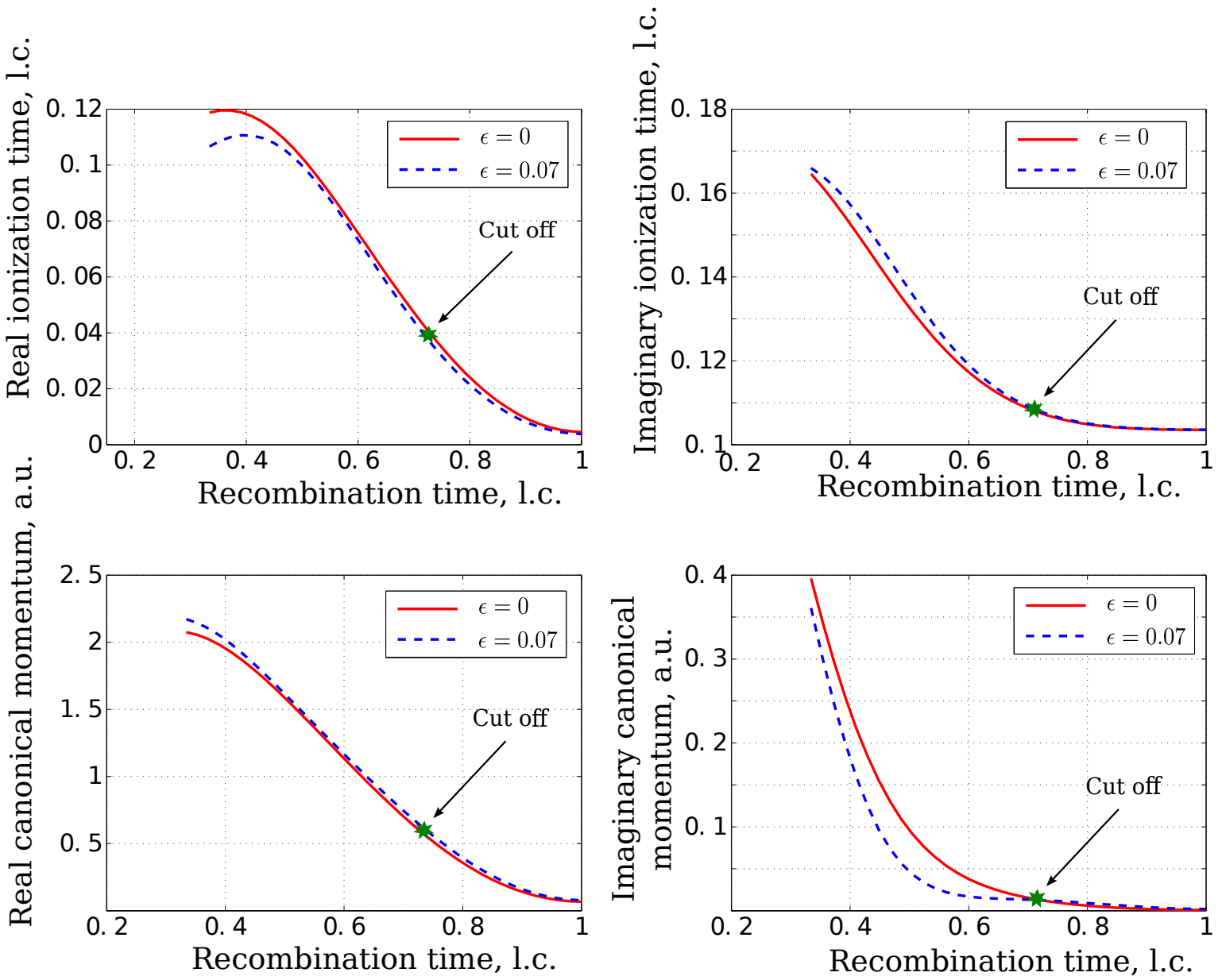

Figure 4.13: Comparison of complex saddle point solutions for ionization time and canonical momentum, obtained with (blue dotted line) and without (red line) perturbative second harmonic. Calculation for the He atom. Parameters of the driving pulse: $I_{0}=10^{14} \mathrm{~W} / \mathrm{cm}^{2}$, $\lambda=800 \mathrm{~nm}$, parameters of the SH: $\lambda_{S H}=400 \mathrm{~nm}, I_{S H}=0.02 I_{0}$, two-color phase $\phi=0$.

Let us plot the deviation of the obtained saddle points from those in the fundamental field, expressed in the percentages of the value of the unperturbed solutions 4.14. Significant deviations are observed for long trajectories, especially for the imaginary canonical momentum, up to 2000\%. Note that, again, this occurs when for the unperturbed trajectory the respective values are close to zero.

The difference between the unperturbed solutions and the solutions with the 
additional SH field are less prominent for short trajectories. For the real ionization time and the real canonical momentum the maximal deviations are about $10 \%$ for the shortest trajectory, while deviations for the trajectories that generate harmonics in the plateau region are less than 5\%. Maximal deviation for imaginary ionization time is $3,5 \%$ for all trajectories. The most significant differences with the unperturbed solution appear again in the imaginary canonical momentum, with up to $150 \%$ deviation in the plateau region. The imaginary momenta are originally rather small in comparison to the real canonical momenta, thus the deviation is large percentage-wise, but not in absolute numbers. 

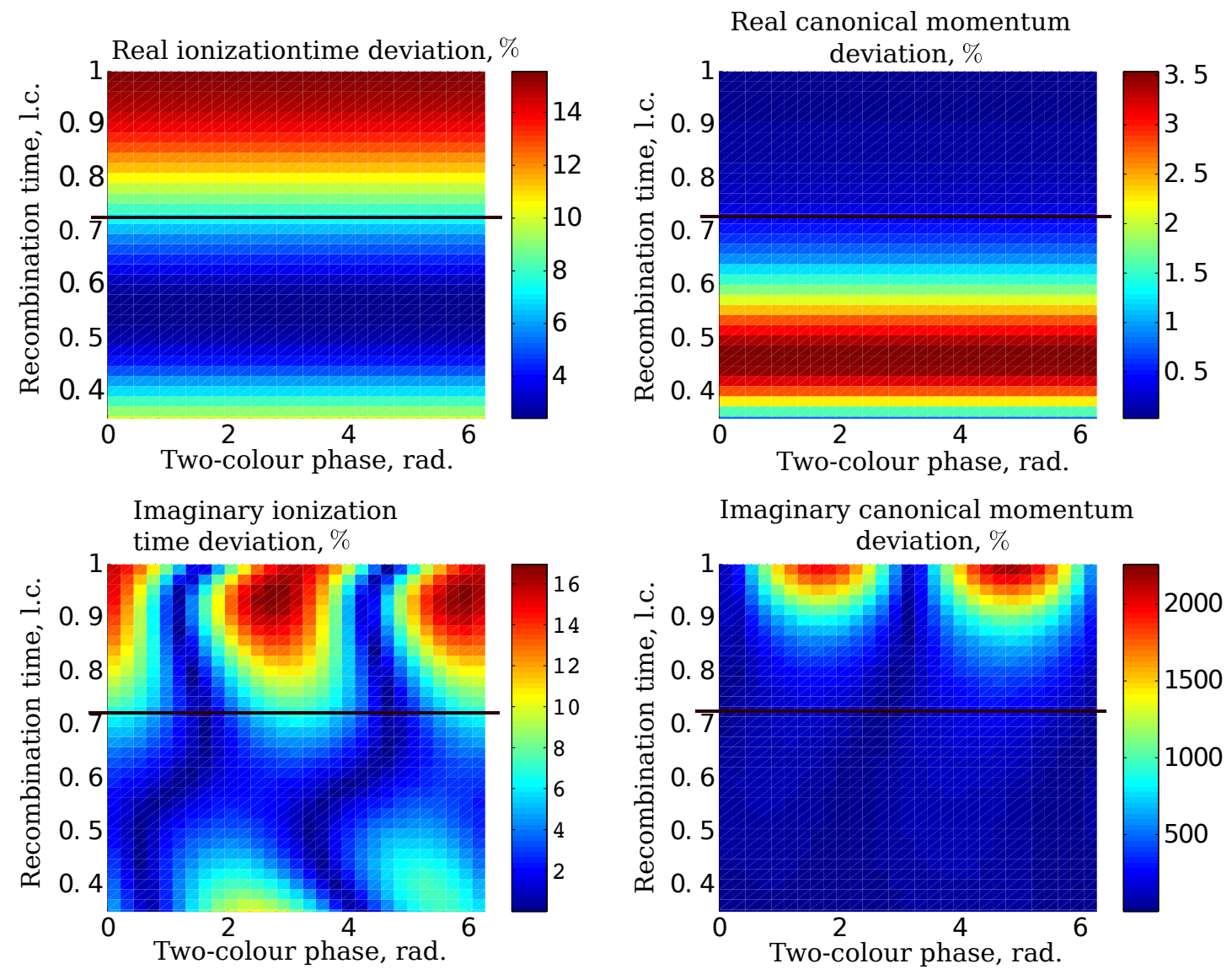

Figure 4.14: The deviation of saddle point solutions, obtained for the case with perturbative second harmonic field in parallel configuration, from the solutions in the fundamental field, expressed in percentages of the value of the unperturbed solutions. Calculation for the He atom. Parameters of the driving pulse: $I_{0}=10^{14} \mathrm{~W} / \mathrm{cm}^{2}$, $\lambda=800 \mathrm{~nm}$, parameters of the SH: $\lambda_{S H}=400 \mathrm{~nm}, I_{S H}=0.02 I_{0}$, two-color phase $\phi=0$. Black line shows the recombination time of the cut off trajectory in the linear field case.

Let us now see how such deviations may affect the HHG amplitude. The key effect here, adequate for atoms, is the correction to the exponential factor that depends on the two-color delay and dominates the modulation of the signal:

$$
|\mathbf{D}(t)|^{2} \sim e^{2 \operatorname{Im} S\left(t_{i}, t_{r}, p, \phi\right)}
$$

We perform the calculations for the parallel configuration in a manner similar 
to the case of the perpendicular fields. After the substitution $k^{\prime}=\frac{p^{\prime}}{A_{0}}, k^{\prime \prime}=$ $\frac{p^{\prime \prime}}{A_{0}}, \varphi_{i}^{\prime}=\omega t_{i}^{\prime}, \varphi_{i}^{\prime \prime}=\omega t_{i}^{\prime \prime}, \varphi_{r}=\omega t_{r}$ the phase 3.27 becomes:

$$
S\left(\varphi_{i}, \varphi_{r}, k, \phi\right)=\frac{A_{0}^{2}}{2 \omega} \int_{\varphi_{i}}^{\varphi_{r}} d \tau\left(k+\frac{A(\tau)}{A_{0}}\right)^{2}+\frac{I_{p}}{\omega}\left(\varphi_{r}-\varphi_{i}\right)
$$

Integrating the expression 4.33 along the contour shown in Figure 3.1 (first, along the imaginary axis from $\varphi_{i}^{\prime \prime}$ to 0 , then along the real axis from $\varphi_{i}^{\prime}$ to $\varphi_{r}$ ) we obtain the amplitude modulation, which is shown inFig.4.15.

The calculated value of the exponent in 4.21 is presented together with its normalization to the maximum of the modulation, for each recombination time.

The position of the optimal two-color phase is found using the same approaches as was done in the previous section for the perpendicular perturbation. Firstly, the optimal delay is found numerically with the help of the Newton method for the zeros of the derivative of the phase 4.33 .

Secondly, we look at how close the two-color delays at which the vector potential of the second field at the moment of ionization is zero to the optimal delays. We make the Taylor expansion near these points and find the maximum point for each recombination time. Finally, we implement so-called 'photo-electron' approach, where the canonical momentum is forced to be real. The results of these procedures are shown in Figure 4.16.

While we see good results for the numerical calculation of the optimal delays, we notice how poorly the photo-electron approach and the Taylor expansion approximation (near the zero points of the vector potential) work. The plot 4.16 shows that optimal conditions for the generating of the maximum signal do not correspond to the zeros of the vector potential of the second harmonic. The minimal difference between the optimal delay, shown with the black curve (numerical calculation) and the delays at which $\mathrm{SH}$ vector potential is zero is $0.5 \mathrm{rad}$, while in the perpendicular configuration the maximum difference was 0.4 for the shortest trajectories and 0.15 on average for other trajectories. For this reason, the Taylor expansion near the zeros of SH vector potential does not give good results. 

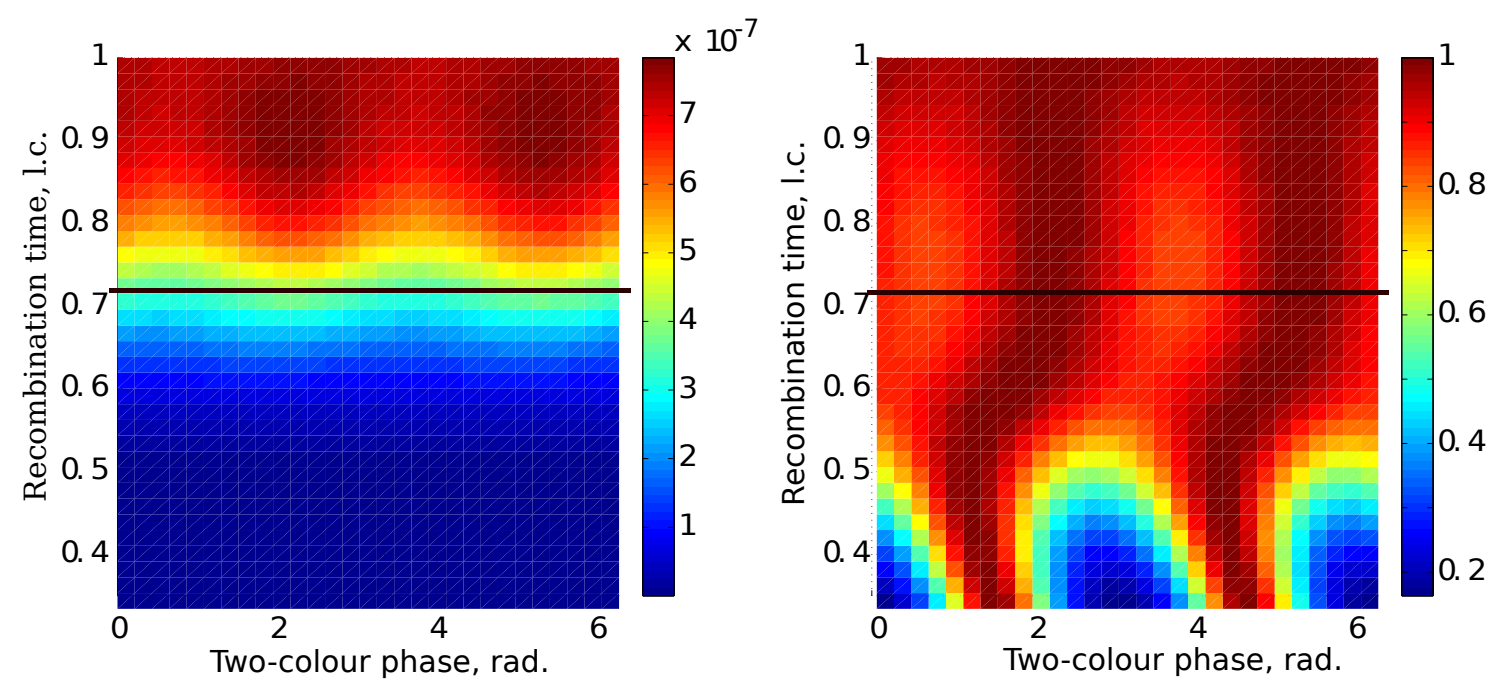

Figure 4.15: The left plot shows the calculated exponential factor 4.32. The right plot slot shows the normalization of this factor to its maximum, for each recombination time. Black lines show the recombination time of the cut off trajectory in the one-color case. Calculation for the He atom. Parameters of the driving pulse: $I_{0}=10^{14}$ $\mathrm{W} / \mathrm{cm}^{2}, \lambda=800 \mathrm{~nm}$, parameters of the $\mathrm{SH}: \lambda_{S H}=400 \mathrm{~nm}$, $I_{S H}=0.02 I_{0}$.

The explanation of why the 'photo-electron' approach does not reproduce correct results for optimal the two-color delays can be found in the Figure that shows the deviation of the saddle point solutions in the two-color case from those obtained in the fundamental field only 4.14. The difference, introduced into the imaginary canonical momentum by the $\mathrm{SH}$ field, has enormous values for many two-color phases, with up to $150 \%$ deviation in the plateau region.

Clearly, for returning trajectories, the imaginary canonical momentum plays important role in parallel configuration of the fields and cannot be discarded from the calculation. 


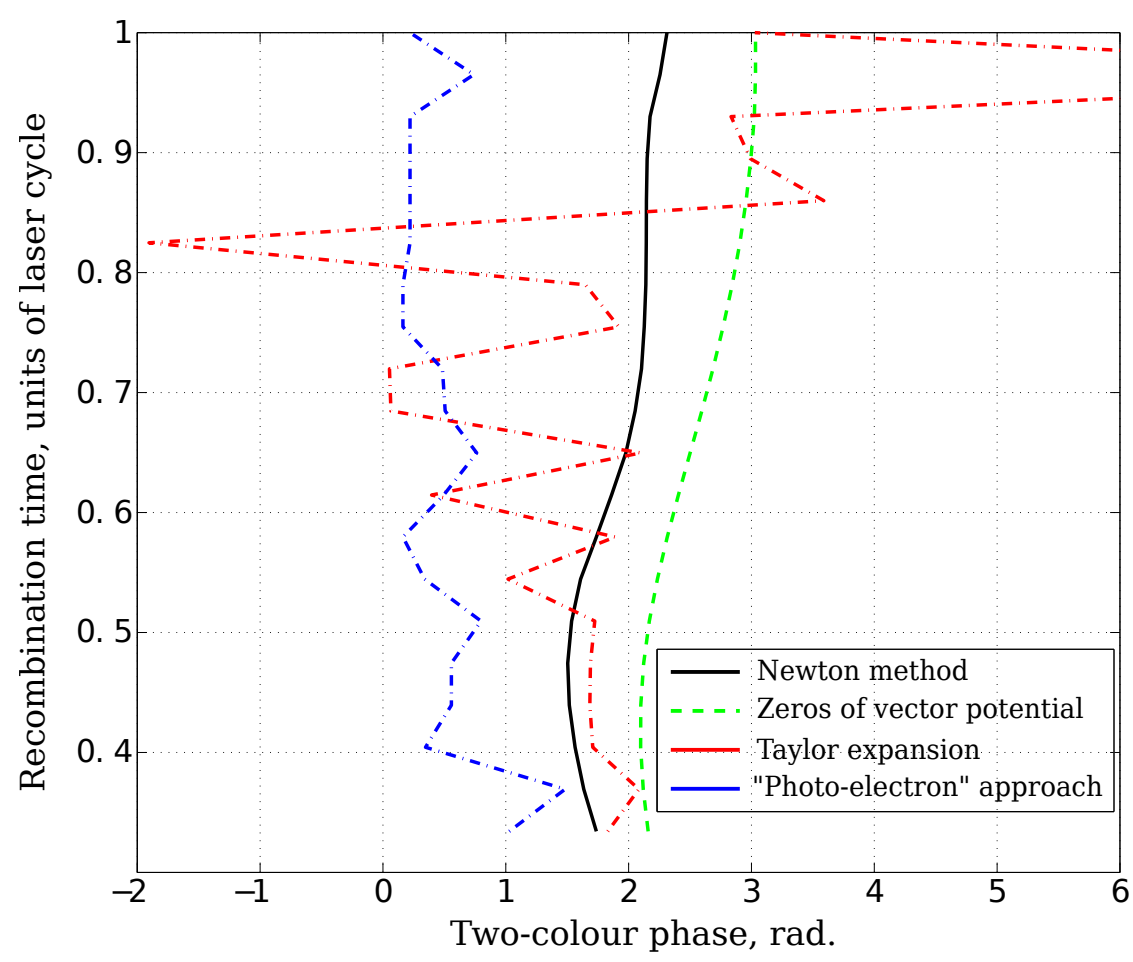

Figure 4.16: Comparison between three calculations of the optimal two-color phase $\phi_{0}$ : black curve - numerical calculation of the optimal twocolor phase, red curve - maximum points of the function 4.32, obtained after its Taylor expansion near zeros of the vector potential (green curve), blue curve - results for the maximum points of the function 4.32, obtained in 'photo-electron' approach. Calculation for the He atom. Parameters of the driving pulse: $I_{0}=10^{14}$ $\mathrm{W} / \mathrm{cm}^{2}, \lambda=800 \mathrm{~nm}$, parameters of the $\mathrm{SH}: \lambda_{S H}=400 \mathrm{~nm}$, $I_{S H}=0.02 I_{0}$.

\subsection{Saddle points for the multicolor fields}

In this section, we consider perpendicular perturbation which includes two fields: the second and third harmonic of the fundamental field. The vector potential of the multicolor perpendicular field is:

$$
\mathbf{A}_{\perp}\left(t, \phi, \phi_{m}\right)=-\epsilon_{2} A_{0} \sin (2 \omega t+\phi) \mathbf{e}_{x}-\epsilon_{3} A_{0} \sin \left(3 \omega t+\phi+\phi_{m}\right) \mathbf{e}_{x}
$$


where $\epsilon_{2}$ and $\epsilon_{3}$ are small parameters for the $2 \omega$ and $3 \omega$ fields, respectively, $\phi$ is the phase delay between the fundamental field and the second harmonic, and $\phi_{m}$ is referred to as the multicolor phase or the multicolor delay.

The saddle point equations now become:

$$
\begin{gathered}
\frac{1}{2}\left(p_{\|}-A_{0} \sin \left(\omega t_{i}\right)\right)^{2}+ \\
+\frac{1}{2}\left(p_{\perp}-\epsilon_{2} A_{0} \sin (2 \omega t+\phi)-\epsilon_{3} A_{0} \sin \left(3 \omega t+\phi+\phi_{m}\right)\right)^{2}+I_{p}=0 \\
\int_{t_{i}}^{t}\left(p_{\|}-A_{0} \sin (\omega \tau)\right) d \tau=0 \\
\int_{t_{i}}^{t}\left(p_{\perp}-\epsilon_{2} A_{0} \sin (2 \omega \tau+\phi)-\epsilon_{3} A_{0} \sin \left(3 \omega \tau+\phi+\phi_{m}\right)\right) d \tau=0
\end{gathered}
$$

The procedure of solving the above saddle point equation 4.35, 4.36 and 4.37 is exactly the same as for the case of the two-color field.

Making the the substitutions $k_{\|}^{\prime}=\frac{p_{\|}^{\prime}}{A_{0}}, k_{\|}^{\prime \prime}=\frac{p_{\|}^{\prime \prime}}{A_{0}}, k_{\perp}^{\prime}=\frac{p_{\perp}^{\prime}}{A_{0}}, k_{\perp}^{\prime \prime}=\frac{p_{\perp}^{\prime \prime}}{A_{0}}, \varphi_{i}^{\prime}=$ $\omega t_{i}^{\prime}, \varphi_{i}^{\prime \prime}=\omega t_{i}^{\prime \prime}, \varphi_{r}=\omega t_{r}$, we obtain from Eqs.(4.36,4.37), after calculating the integral in Eq.(4.6),

$$
\begin{gathered}
k_{\|}^{\prime}=\frac{k_{\|}^{\prime \prime}\left(\varphi_{r}-\varphi_{i}^{\prime}\right)}{\varphi_{i}^{\prime \prime}}+\frac{\sin \varphi_{i}^{\prime} \sinh \varphi_{i}^{\prime \prime}}{\varphi_{i}^{\prime \prime}} \\
k_{\|}^{\prime \prime}=\frac{\varphi_{i}^{\prime \prime} \cos \varphi_{i}^{\prime} \cosh \varphi_{i}^{\prime \prime}-\varphi_{i}^{\prime} \cos \varphi_{r}-\left(\varphi_{r}-\varphi_{i}^{\prime}\right) \sin \varphi_{i}^{\prime} \sinh \varphi_{i}^{\prime \prime}}{\varphi_{i}^{\prime \prime 2}+\left(\varphi_{r}-\varphi_{i}^{\prime}\right)^{2}} \\
k_{\perp}^{\prime}=\frac{k_{\perp}^{\prime \prime}\left(\varphi_{r}-\varphi_{i}^{\prime}\right)}{\varphi_{i}^{\prime \prime}}+\frac{\epsilon_{2}}{2} \frac{\sin \left(2 \varphi_{i}^{\prime}+\phi\right) \sinh 2 \varphi_{i}^{\prime \prime}}{\varphi_{i}^{\prime \prime}}+ \\
+\frac{\epsilon_{3}}{3} \frac{\sin \left(3 \varphi_{i}^{\prime}+\phi+\phi_{m}\right) \sinh 3 \varphi_{i}^{\prime \prime}}{\varphi_{i}^{\prime \prime}}
\end{gathered}
$$




$$
\begin{aligned}
& k_{\perp}^{\prime \prime}= \\
& =\frac{\epsilon_{2}}{2} \frac{\varphi_{i}^{\prime \prime} \cos \left(2 \varphi_{i}^{\prime}+\phi\right) \cosh 2 \varphi_{i}^{\prime \prime}-\varphi_{i}^{\prime} \cos \left(2 \varphi_{r}+\phi\right)-\left(\varphi_{r}-\varphi_{i}^{\prime}\right) \sin \left(2 \varphi_{i}^{\prime}+\phi\right) \sinh 2 \varphi_{i}^{\prime \prime}}{\varphi_{i}^{\prime 2}+\left(\varphi_{r}-\varphi_{i}^{\prime}\right)^{2}}+ \\
& +\frac{\epsilon_{3}}{3} \frac{1}{\varphi_{i}^{\prime \prime 2}+\left(\varphi_{r}-\varphi_{i}^{\prime}\right)^{2}}\left(\varphi_{i}^{\prime \prime} \cos \left(3 \varphi_{i}^{\prime}+\phi+\phi_{m}\right) \cosh 3 \varphi_{i}^{\prime \prime}-\varphi_{i}^{\prime} \cos \left(3 \varphi_{r}+\phi+\phi_{m}\right)-\right. \\
& \left.-\left(\varphi_{r}-\varphi_{i}^{\prime}\right) \sin \left(3 \varphi_{i}^{\prime}+\phi+\phi_{m}\right) \sinh 3 \varphi_{i}^{\prime \prime}\right)
\end{aligned}
$$

As in the previous cases, we construct the surface:

$$
F_{1}^{2}\left(\varphi_{i}^{\prime}, \varphi_{i}^{\prime \prime}, \varphi_{r}, \phi, \phi_{m}\right)+F_{2}^{2}\left(\varphi_{i}^{\prime}, \varphi_{i}^{\prime \prime}, \varphi_{r}, \phi, \phi_{m}\right)=0
$$

where $F_{1}$ and $F_{2}$ are the real and imaginary parts of the left part of the equation 4.35. The values of real $\varphi_{i}^{\prime}$ and imaginary $\varphi_{i}^{\prime \prime}$ times are found as the solutions of the equation Eq.(4.42). Canonical momentum is calculated according to Eqs.(4.38, 4.39, 4.40) and Eq.(4.41). The obtained saddle-point solutions are shown in Figures 4.17 and 4.18. 

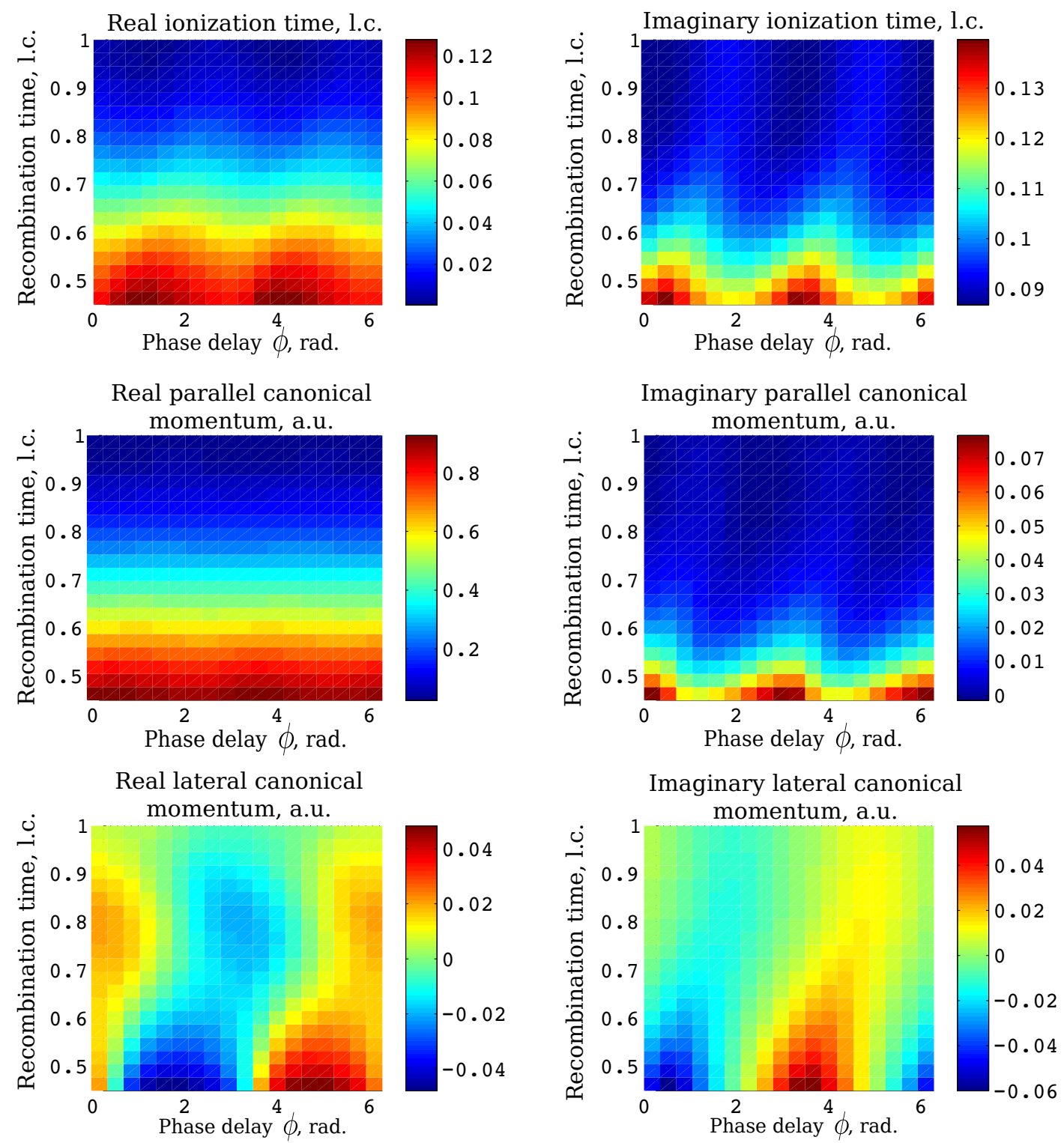

Figure 4.17: Complex saddle points solutions for the ionization time and canonical momentum for the combination of the fundamental second harmonic and the third harmonic fields. Calculation for the He atom. Parameters of the driving pulse: $I_{0}=10^{14} \mathrm{~W} / \mathrm{cm}^{2}$, $\lambda=1600 \mathrm{~nm}$, parameters of the perturbation: $\lambda_{S H}=800 \mathrm{~nm}$, $I_{S H}=0.02 I_{0}, \lambda_{T H}=400 \mathrm{~nm}, I_{T H}=0.01 I_{0}$, delay between the second and the third harmonic $\phi_{m}=0 \mathrm{rad}$. 

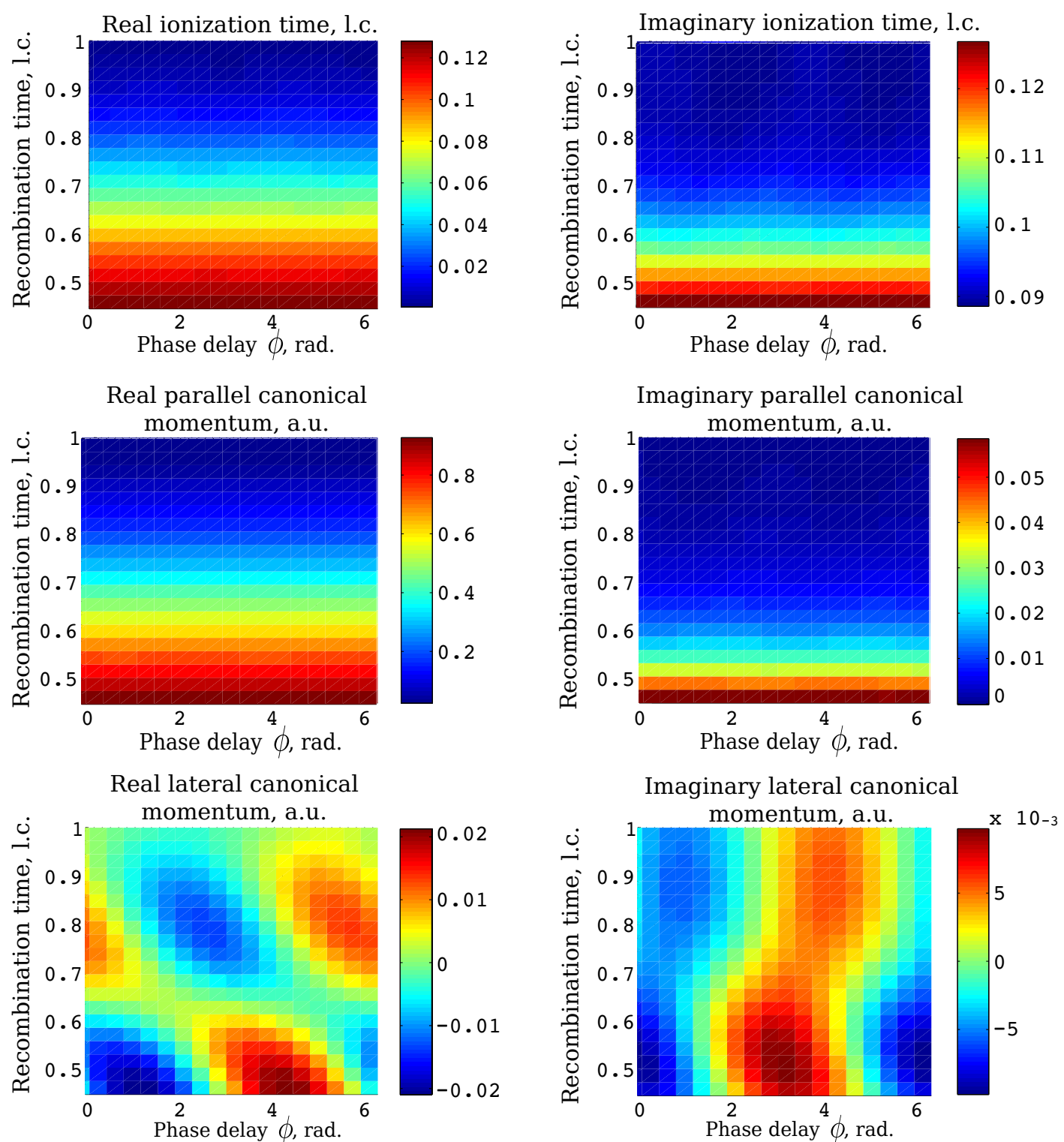

Figure 4.18: Complex saddle points solutions for the ionization time and canonical momentum for the combination of the fundamental, the second harmonic, and the third harmonic fields. Calculation for the He atom. Parameters of the driving pulse: $I_{0}=10^{14}$ $\mathrm{W} / \mathrm{cm}^{2}, \lambda=1600 \mathrm{~nm}$, parameters of the perturbation: $\lambda_{S H}=800$ $\mathrm{nm}, I_{S H}=0.02 I_{0}, \lambda_{T H}=400 \mathrm{~nm}, I_{T H}=0.01 I_{0}$, delay between second and third harmonic $\phi_{m}=2.1 \mathrm{rad}$.

The difference between the figures 4.17 and 4.18 is the value of the phase between the second and the third perturbative harmonics: $\phi_{m}=0 \mathrm{rad}$ for the 
plots in Fig.4.17 and $\phi_{m}=2,1 \mathrm{rad}$ for the plots in Fig. 4.18. We note the difference between these two cases, especially for the ionization time and the lateral canonical momentum.

Let us now look at the comparison between the saddle point solutions in the linear field and the multicolor field, for both situations. It is shown in Figures 4.19 and 4.20 .
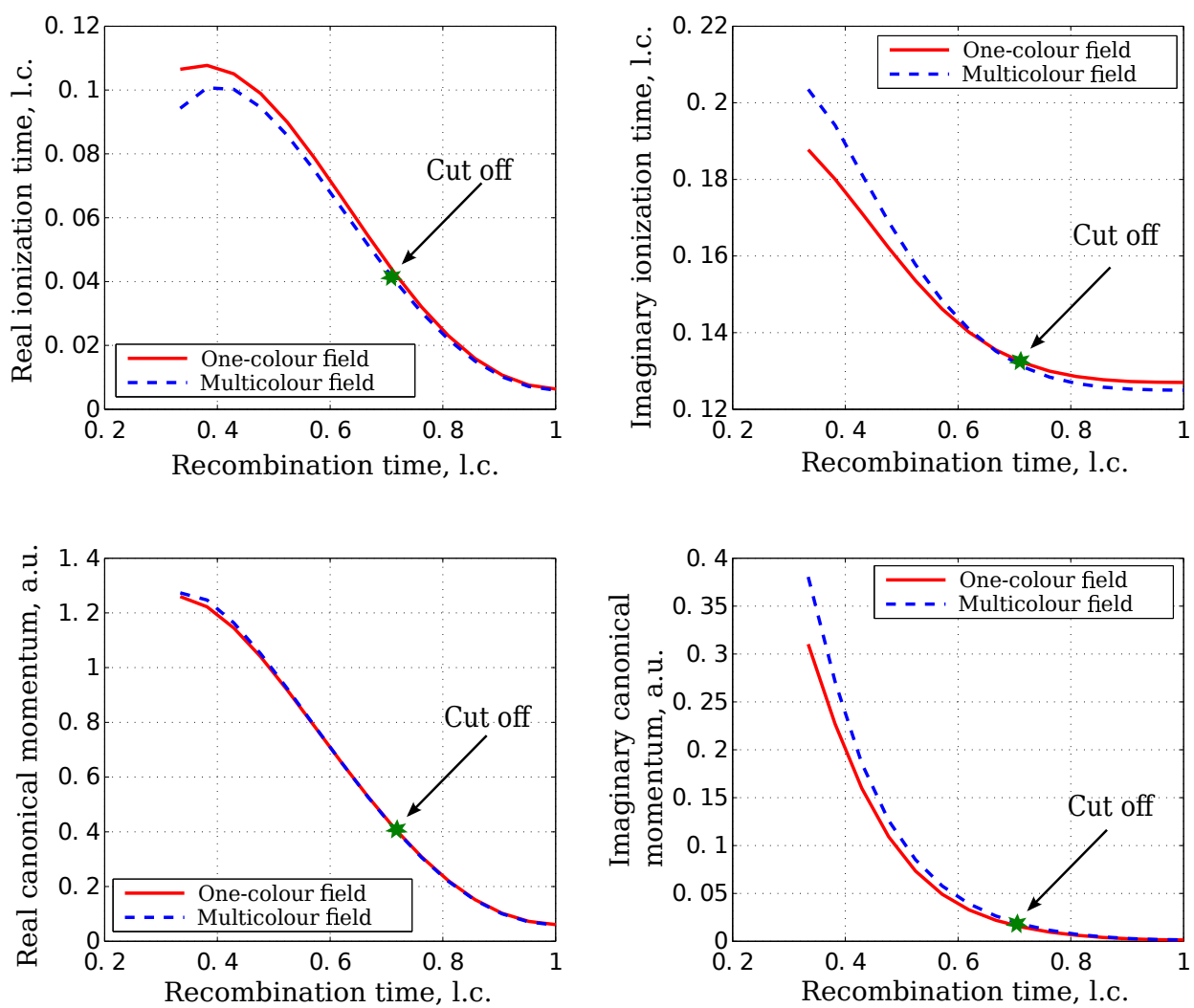

Figure 4.19: Comparison of the complex saddle points solutions for the ionization time and the canonical momentum, obtained with (blue dotted line) and without (red line) perturbation field. Calculation for the He atom. Parameters of the driving pulse: $I_{0}=10^{14}$ $\mathrm{W} / \mathrm{cm}^{2}, \lambda=1600 \mathrm{~nm}$, parameters of the perturbation: $\lambda_{S H}=800$ $\mathrm{nm}, I_{S H}=0.02 I_{0}, \lambda_{T H}=400 \mathrm{~nm}, I_{T H}=0.01 I_{0}$, delay between the second and the third harmonic $\phi_{m}=0 \mathrm{rad}$. 

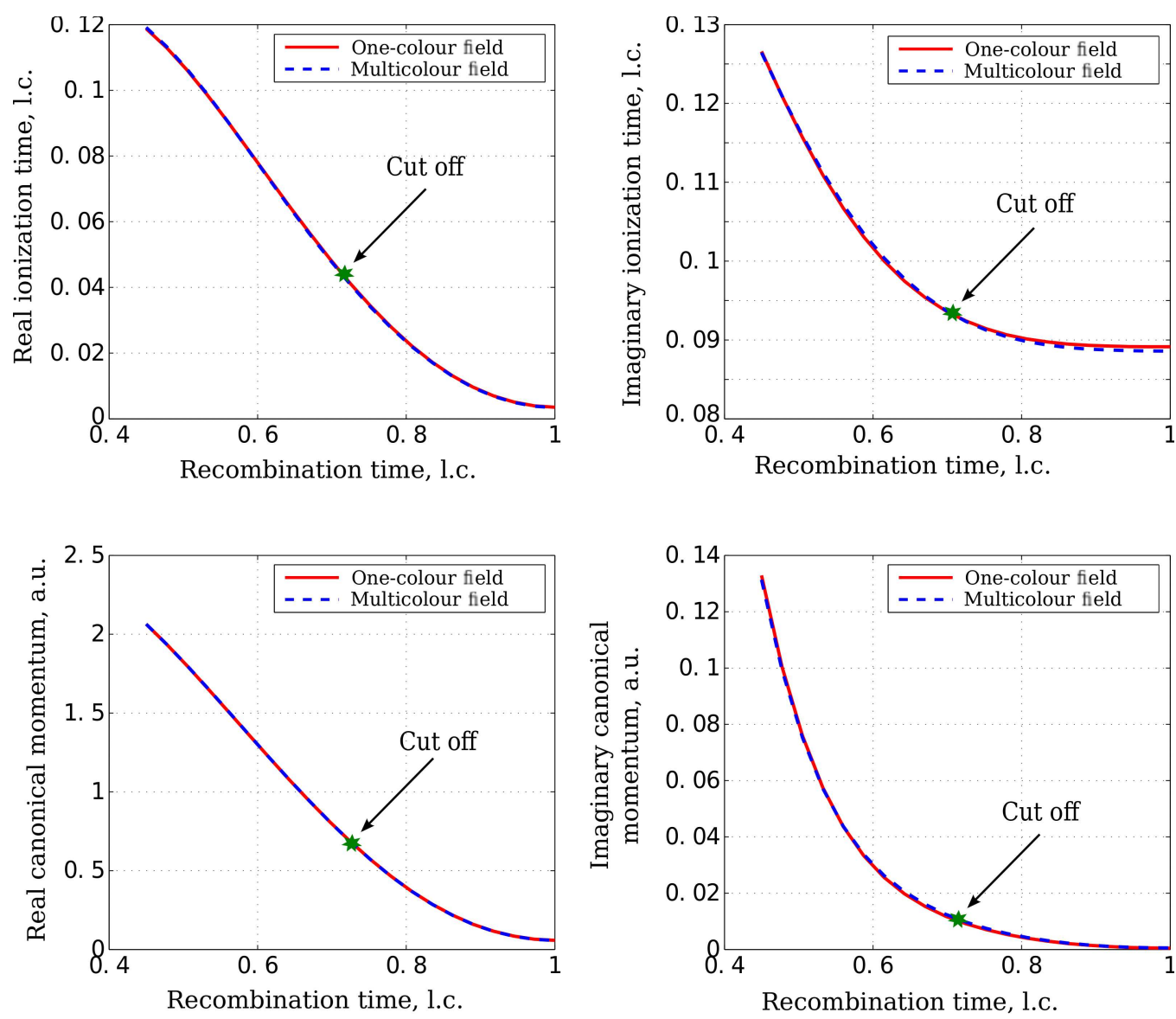

Figure 4.20: Comparison of complex saddle points solutions for the ionization time and the canonical momentum, obtained with (blue dotted line) and without (red line) the perturbation field. Calculation for the He atom. Parameters of the driving pulse: $I_{0}=10^{14} \mathrm{~W} / \mathrm{cm}^{2}$, $\lambda=1600 \mathrm{~nm}$, parameters of the perturbation: $\lambda_{S H}=800 \mathrm{~nm}$, $I_{S H}=0.02 I_{0}, \lambda_{T H}=400 \mathrm{~nm}, I_{T H}=0.01 I_{0}$, delay between second and third harmonic $\phi_{m}=2,1 \mathrm{rad}$.

The difference between the saddle points solutions for the two values of the multicolor phase $\phi_{m}$ is noticeable, when we examine the deviation of the saddle point solutions from the linear field case. These are presented in Figures 4.21 and 4.22 .

For the multicolor phase $\phi_{m}=2,1 \mathrm{rad}$ we generally observe small deviations: less than $1 \%$ for both real and imaginary parts of the ionization time and for the real canonical momentum and. The largest deviation in this case is for the 
imaginary canonical momentum, of about $7 \%$ for the shortest trajectory, and again less than $1 \%$ for the plateau trajectories.

We observe higher deviations for the case with the multicolor phase $\phi_{m}=0$ : about $7 \%$ for the real part of ionization time, less than $9 \%$ for the imaginary ionization time, less than $3 \%$ for real canonical momentum and, and again the largest deviation of about $20 \%$ for the imaginary canonical momentum.

Let us look at the modulation of the amplitude of the harmonic spectrum, introduced by the exponential factor:

$$
|\mathbf{D}(t)|^{2} \sim e^{2 \operatorname{Im} S\left(\varphi_{i}, \varphi_{r}, k_{\|}, p_{\perp}, \phi, \phi_{m}\right)}
$$

The phase $S\left(\varphi_{i}, \varphi_{r}, k_{\|}, p_{\perp}, \phi, \phi_{m}\right)$ is written as:

$$
\begin{gathered}
S\left(\varphi_{i}, \varphi_{r}, k_{\|}, k_{\perp}, \phi, \phi_{m}\right)=\frac{1}{2} \frac{A_{0}^{2}}{\omega} \int_{\varphi_{i}}^{\varphi_{r}} d t\left(k_{\|}+\frac{A_{\text {par }}(\tau)}{A_{0}}\right)^{2}+ \\
+\frac{1}{2} \frac{A_{0}^{2}}{\omega} \int_{\varphi_{i}}^{\varphi_{r}} d t\left(k_{\perp}+\frac{A_{p e r}(\tau)}{A_{0}}\right)^{2}+\frac{I_{p}}{\omega}\left(\varphi_{r}-\varphi_{i}\right)
\end{gathered}
$$

We integrate the expression 4.44 along the contour, shown in the figure 3.1. The normalization of Eq. (4.43) for each recombination time to the maximum of the modulation is presented in Figure 4.23 for the values of the multicolor phase $\phi_{m}=0 \mathrm{rad}$ and $\phi_{m}=2,1 \mathrm{rad}$.

We can now compare the positions of the optimal phase $\phi_{\text {opt }}$ for the two different values of the multicolor phase $\phi_{m}$. We find the optimal delay numerically, as described in the section 4.1.1. Then, we will also calculate the modulation amplitude within the classical approach. 

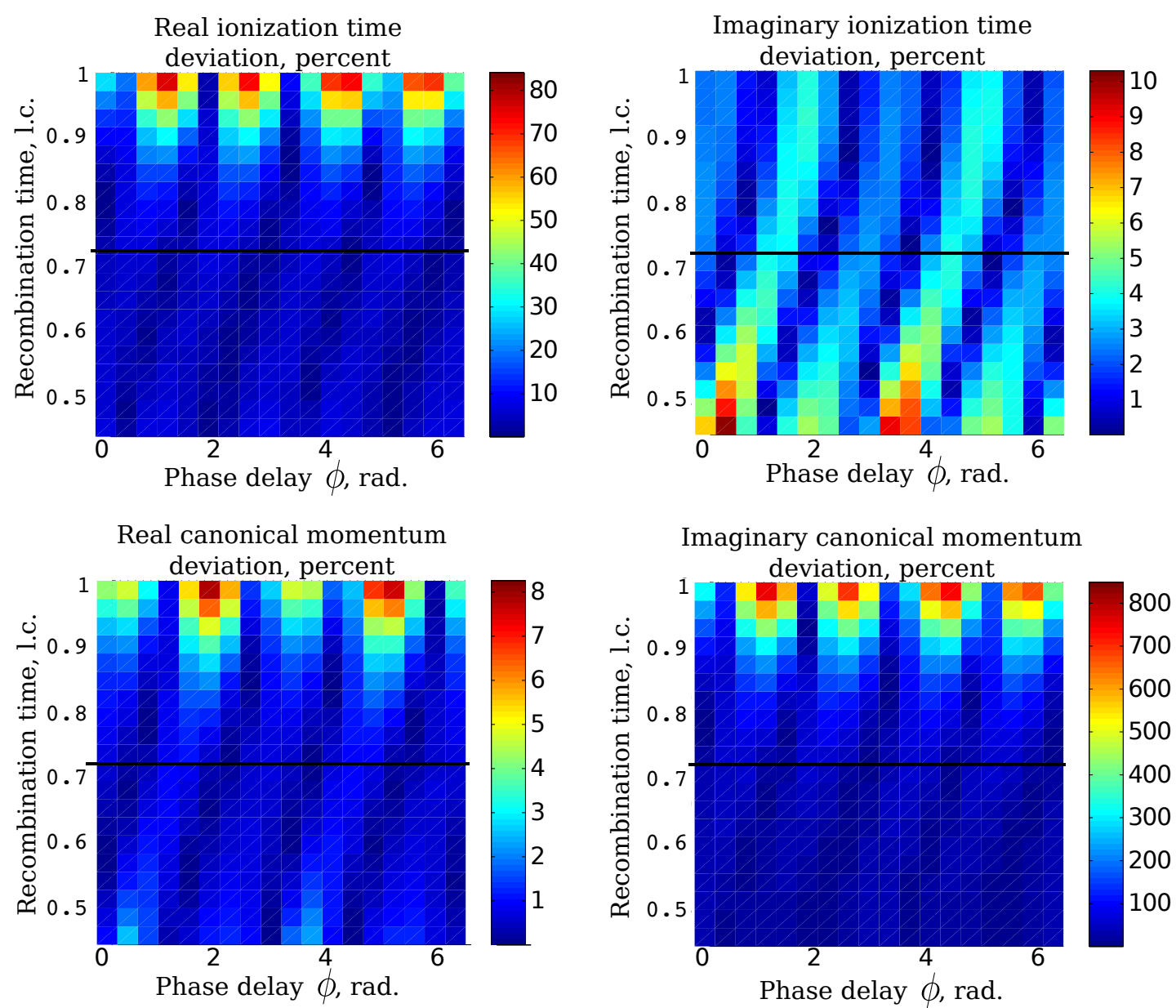

Figure 4.21: Deviations of the saddle point solutions, obtained for the case with the perturbative multicolor field, from the solutions in the fundamental field only, expressed in percentages of the value of the unperturbed solutions. Calculation for the He atom. Parameters of the driving pulse: $I_{0}=10^{14} \mathrm{~W} / \mathrm{cm}^{2}, \lambda=800 \mathrm{~nm}$, parameters of the perpendicularly polarized multicolor field: $\lambda=1600 \mathrm{~nm}$, parameters of the perturbation: $\lambda_{S H}=800 \mathrm{~nm}, I_{S H}=0.02 I_{0}$, $\lambda_{T H}=400 \mathrm{~nm}, I_{T H}=0.01 I_{0}$, delay between second and third harmonic $\phi_{m}=0$ rad. Black line shows the recombination time of the cut off trajectory in the linear field case. 

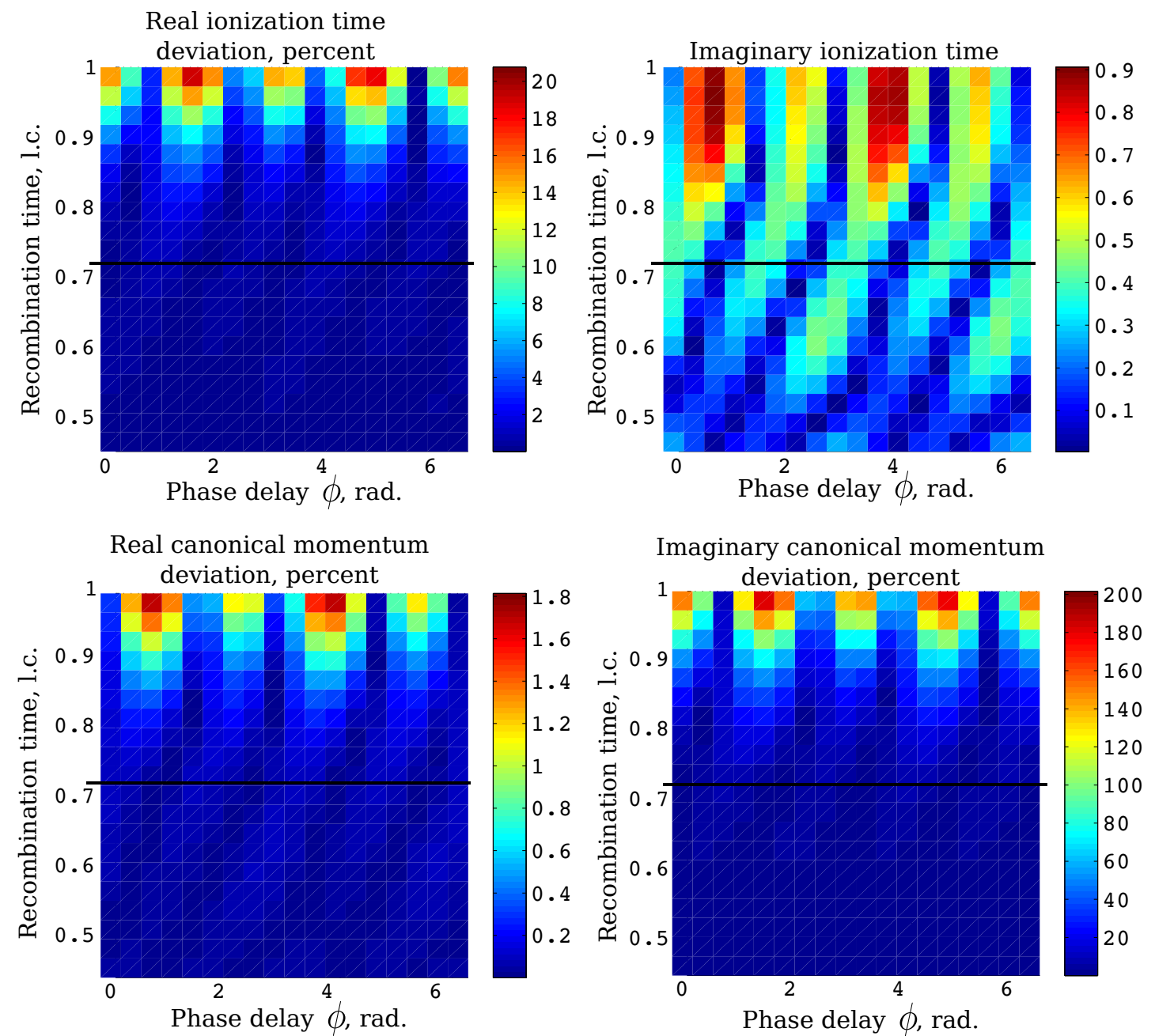

Figure 4.22: Deviation of the saddle point solutions, obtained for the case with the perturbative multicolor field, from the solutions in the fundamental field only, expressed in percentages of the value of the unperturbed solutions. Calculation for the He atom. Parameters of the driving pulse: $I_{0}=10^{14} \mathrm{~W} / \mathrm{cm}^{2}, \lambda=1600 \mathrm{~nm}$, parameters of the perturbation: $\lambda_{S H}=800 \mathrm{~nm}, I_{S H}=0.02 I_{0}, \lambda_{T H}=400$ $\mathrm{nm}, I_{T H}=0.01 I_{0}$, delay between second and third harmonic $\phi_{m}=2,1 \mathrm{rad}$. Black line shows the recombination time of the cut off trajectory in the linear field case. 

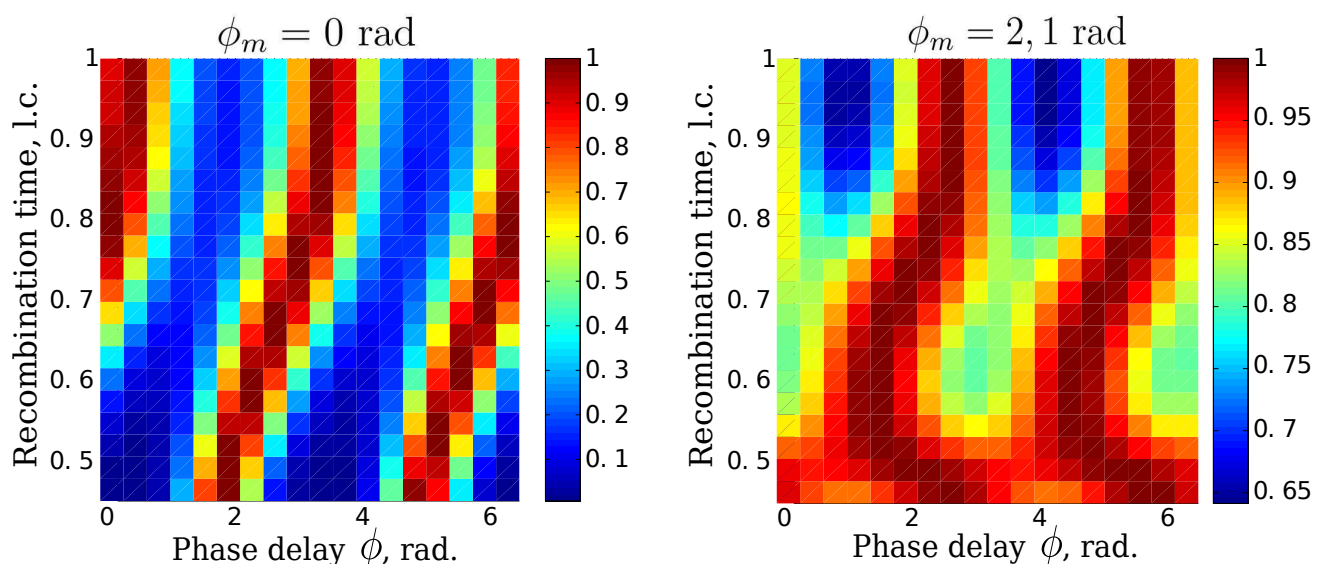

Figure 4.23: The plot shows normalization of Eq. (4.43) to its maximum value for each recombination time. Calculation for the He atom. Parameters of the driving pulse: $I_{0}=10^{14} \mathrm{~W} / \mathrm{cm}^{2}, \lambda=1600 \mathrm{~nm}$, parameters of the perturbation: $\lambda_{S H}=800 \mathrm{~nm}, I_{S H}=0.02 I_{0}$, $\lambda_{T H}=400 \mathrm{~nm}, I_{T H}=0.01 I_{0}$, delay between second and third harmonic $\phi_{m}=0 \mathrm{rad}$ on the left plot and $\phi_{m}=2,1 \mathrm{rad}$ on the right plot.

The classical gate is defined as $G_{c l}\left(\phi, \phi_{m}\right)=\exp \left[-\frac{1}{2} v_{\perp}^{2} \tau\right]$, where $\tau \equiv t_{i}^{\prime \prime}$ and $v_{\perp}$ is the lateral velocity of the electron. It is derived from the classical condition on the zero lateral displacement of an the electron after ionization:

$$
\int_{t_{i}^{\prime}}^{t_{r}} v_{\perp} d t=\int_{t_{i}^{\prime}}^{t_{r}}\left(k_{\perp}^{c l}+A_{\perp}(t)\right) d t=0
$$

where $k_{\perp}^{c l}$ is a classical lateral canonical momentum. It has no imaginary part, because the classical return condition implies no imaginary parts for the ionization and the recombination times, as well as the absence of the tunnelling barrier (the trajectory starts and ends at the origin).

The results of the calculation of the optimal phases $\phi_{\text {opt }}$ for the two cases of the multicolor delays $\phi_{m}=0 \mathrm{rad}$ and $\phi_{m}=2,1 \mathrm{rad}$ are presented in Figure 4.24. Additionally, the plots in Figure 4.24 show such phases $\phi_{0}$, for which the vector-potentials of the perpendicular fields at the instant of ionization are 
zero:

$$
A_{\perp}\left(\phi_{0}\right)=0, A_{2 \omega}\left(\phi_{0}\right)=0, A_{3 \omega}\left(\phi_{0}\right)=0
$$

We see significant difference between the values of the optimal delays $\phi_{\text {opt }}$ for the two different multicolor phases $\phi_{m}$. Most importantly, the position of the optimal phase $\phi_{\text {opt }}$ for the multicolor delay $\phi_{m}=0 \mathrm{rad}$ found by numerical calculation and as a maximum of classical gate coincide. However, it is clear, that in the case of $\phi_{m}=2,1 \mathrm{rad}$ the simplified classical approach is inapplicable. The consequences of this fact are described further in the section 5.2.2.

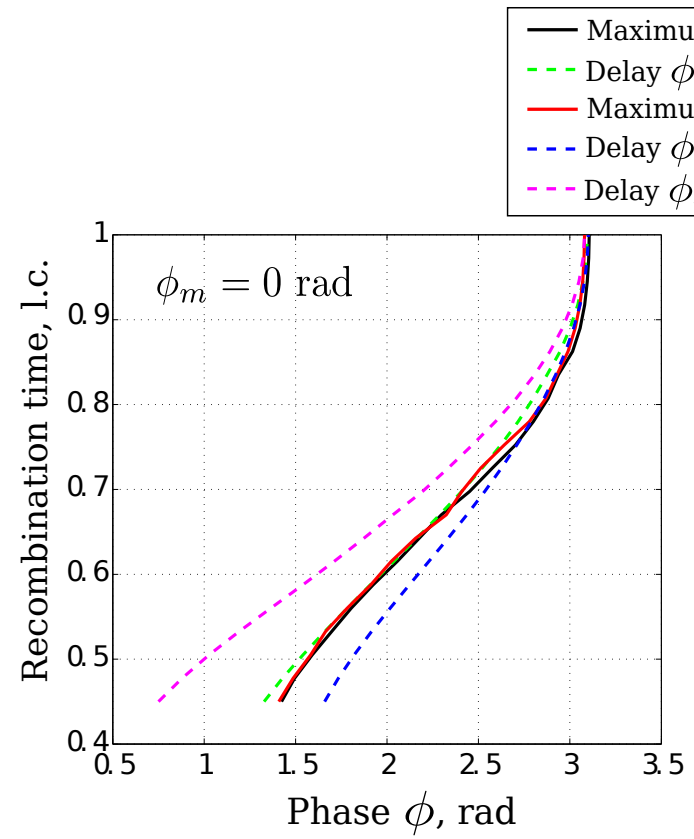

Figure 4.24: Comparison between the optimal phases $\phi$ for the two values of the multicolor delay $\phi_{m}=0 \mathrm{rad}$ (left plot) and $\phi_{m}=2,1 \mathrm{rad}$ (right plot): black curve - numerical calculation of the optimal phase $\phi$, red curve - maximum of the classical gate $G_{c l}$, green dotted curve - phases $\phi$, at which $A_{\perp}(\phi)=0$, blue dotted curve phases $\phi$, at which $A_{2 \omega}(\phi)=0$, magenta dotted curve - phases $\phi$, at which $A_{3 \omega}(\phi)=0$. Calculation for the He atom. Parameters of the driving pulse: $I_{0}=10^{14} \mathrm{~W} / \mathrm{cm}^{2}, \lambda=1600 \mathrm{~nm}$, parameters of the perturbation: $\lambda_{S H}=800 \mathrm{~nm}, I_{S H}=0.02 I_{0}, \lambda_{T H}=400$ $\mathrm{nm}, I_{T H}=0.01 I_{0}$. 


\section{Gating with two-color fields for the reconstruction of the parameters of electron trajectories.}

In the previous chapter we have described the effect of the perturbative fields on the parameters of the electron trajectories. The properties of two-dimensional high harmonic generation spectra can now be used for the reverse analysis: what does the observed multicolor HHG spectrum tell us about the processes of ionization, propagation and recombination?

The gating effect of the second harmonic field on the electron trajectories is implemented to study the ionization from atoms and molecules. In this chapter, we will consider the procedure for extracting real part of the ionization time, presented in [93]. We will also study the two options for obtaining the imaginary part of the ionization time from the two-dimensional spectrum $[154,162]$.

\subsection{Reconstruction of time of exit from the tunnelling barrier}

The idea of reconstructing the time of exit from the tunnelling barrier using the two-dimensional HHG spectra obtained in the two-color field was first presented in Ref. [93]. It was discussed in the literature review in the section 2.3. 
Here we reiterate the idea of the 'gating technique', because it is important as an illustration of the main principle used for reconstructing the ionization times. Relevant derivations of the expression for the gates and for the reconstruction procedures can be found in [93] (supplementary material).

The schematic representation of the two-color fields, when the second harmonic has perpendicular polarization relative to the fundamental field, is shown in Figure 2.4. The second harmonic field negligibly perturbs the ionization times, as was shown in Section 4.1.1. After ionization, it acts on the electron in the lateral direction, modifying its trajectory. As a result, the electron may miss the ion after it returns to the vicinity of the core, due to displacement along the transverse direction 2.5. Thus, the harmonic generation signal is suppressed. However, when the phase delay between fundamental and SH fields is optimal, the electron trajectory ends at the coordinates of the ion, in both lateral and longitudinal directions, leading to recombination and emission of harmonics.

As the parameters of electron trajectories vary from one harmonic number to another harmonic number, so does the value of the optimal delay. Thus, the second harmonic field acts as 'gate' on the parameters of the electron trajectories, including the ionization time. This gate is referred to as the "displacement gate", and its expression is [93]:

$$
\begin{gathered}
G_{y}\left(t_{r}, t_{i}, \phi\right)=e^{-\frac{1}{2} v_{0 y}^{2}\left(t_{r}, t_{i}, \phi\right) t_{i}^{\prime \prime}} \\
v_{0 y}\left(t_{r}, t_{i}, \phi\right)=-\epsilon \frac{F_{0}}{\omega}\left(\sin \left(2 \omega t_{i}+\phi\right)+\frac{\cos \left(2 \omega t_{r}+\phi\right)-\cos \left(2 \omega t_{i}+\phi\right)}{2 \omega\left(t_{r}-t_{i}\right)}\right)
\end{gathered}
$$

where $G_{y}\left(t_{r}, t_{i}, \phi\right)$ is the displacement gate and $v_{0 y}\left(t_{r}, t_{i}, \phi\right)$ is the initial lateral velocity of the electron. Lateral displacement, acquired by the electron due to the interaction with the SH field, is compensated by the initial lateral velocity $v_{0 y}\left(t_{r}, t_{i}, \phi\right)$, which was obtained from the condition on the zero lateral displacement:

$$
\int_{t_{i}}^{t_{r}} v_{y} d t=0
$$

The second harmonic field breaks the symmetry between the subsequent half- 
cycles of the fundamental field. In these conditions, the interference of the XUV light, emitted during each half-cycle, leads to the appearance of even harmonics in the HHG spectrum. The maximum ratio between the intensities of the adjacent even and odd harmonics is observed when the symmetry breaking leads to the maximal lateral electron velocity upon recombination. Indeed, in this case the asymmetry between the two subsequent half-cycles is maximized.

Therefore, the gate associated with the recombination time is called the "velocity gate" [93]. It is written as:

$G_{v}\left(t_{r}, t_{i}, \phi\right)=\frac{F_{0}}{\omega \sqrt{2\left(N \omega-I_{p}\right)}}\left(\sin \left(2 \omega t_{r}+\phi\right)+\frac{\cos \left(2 \omega t_{r}+\phi\right)-\cos \left(2 \omega t_{i}+\phi\right)}{2 \omega\left(t_{r}-t_{i}\right)}\right)$

Once the optimal phases $\phi_{\text {opt }}$ are found from the experiment for each harmonic number, solving the equations for the optimal phases for both the displacement 5.1 and the velocity 5.4 gates, it is possible to obtain the values of the real ionization time $t_{i}^{\prime}$ and the real recombination time $t_{r}$. The result of the reconstruction and its comparison with theoretical calculation is presented in the paper [93] for He, illustrating excellent agreement.

Since there are only two equations $(5.1,5.4)$, it is possible to reconstruct only two variables. The remaining variable is the imaginary part of the ionization time. In the described reconstruction, it is assumed to be known and its value is obtained from the analytical strong-field ionization theory [72]. However, since the imaginary ionization time determines the ionization probability, i.e. the population of the given quantum trajectory, it only affects the contrast in the harmonic signal modulation, not the position of the maxima and minima. Therefore, the reconstruction procedure for the real times is not affected by it.

In order to extract the imaginary part of the ionization time from the experiment, the number of equations available for our analysis should be increased, or the equations should be changed in order to make the imaginary ionizations times more important for the gating. The former can be done by introducing another control parameter into the experimental scheme, i.e. by 
adding another color. The latter can be done by changing the direction of the perturbative field, specifically, by considering parallel perturbation instead of perpendicular. These two options are considered below.

\subsection{Reconstruction of the imaginary part of the ionization time}

In order to reconstruct some characteristic of the electron trajectory from the experiment in multicolor fields, we have to have the capability of fine tuning this characteristic by changing the experimental parameters. In other words, the value of this characteristic, and how it is changing from one harmonic number to another, has to play important role in the formation of the two-dimensional HHG signal landscape. In the previous example, when the perturbative second harmonic affected the electron trajectory after ionization, the imaginary ionization time determined only the contrast of the gate. What can be done in order to change it? Is it possible to single out the effect of the imaginary ionization time on the position of the optimal delay, or change the experiment in such a way that the electron is noticeably affected by the perturbation in the classically forbidden region?

\subsubsection{Attosecond tunnelling interferometry}

One of the possible answers to the question above was given by the setup and the reconstruction procedure developed in our paper [154].

Instead of using the second harmonic of the fundamental field as a perpendicular perturbation, it is now applied in parallel direction. As we have seen in 4.2 , in this field geometry the saddle points change more significantly, and this is especially true for the imaginary part of ionization time. It happens due to the effect of the additional field on the tunnelling process. Unlike the case of the second harmonic with perpendicular polarization, when only the angle of ionization changes very slightly, the parallel SH field distorts the tunnelling barrier, bending it up or down in comparison with the single-color field, which 
leads to big deviations of the trajectory parameters, especially the canonical momentum and the imaginary ionization time (see chapter 4.2 of the Thesis).

The principle of the attosecond tunnelling interferometer is illustrated in Figure 5.1. Ionization bursts happen twice during the period of the fundamental laser field. Without the second harmonic, the two consecutive half-cycles are symmetric: the width of the barrier, corresponding to the same real ionization time and the same harmonic photon energy, is the same for both half-cycles. However, with the presence of the parallel SH field, during one half-cycle the electron "sees" a thicker barrier, than during the second half-cycle. The electron trajectory, which corresponds to a certain harmonic photon energy, will have a different length in classically forbidden region for the subsequent half-cycles. These two paths represent the two arms of the interferometer. The relative phase between the two paths is the difference between the phases accumulated under the potential barriers in the two subsequent half-cycles.

The reconstruction procedure of imaginary part of ionization time, proposed in [154], is based on the retrieval of the phase difference between the interferometer paths and its analysis is based on using the SFA. As a result of a broken symmetry between two consecutive half-cycles both odd and even harmonics are generated. The complex phase $\sigma$ can be retrieved from the experimental harmonic spectrum by analyzing the intensities of neighboring even and add harmonics. 


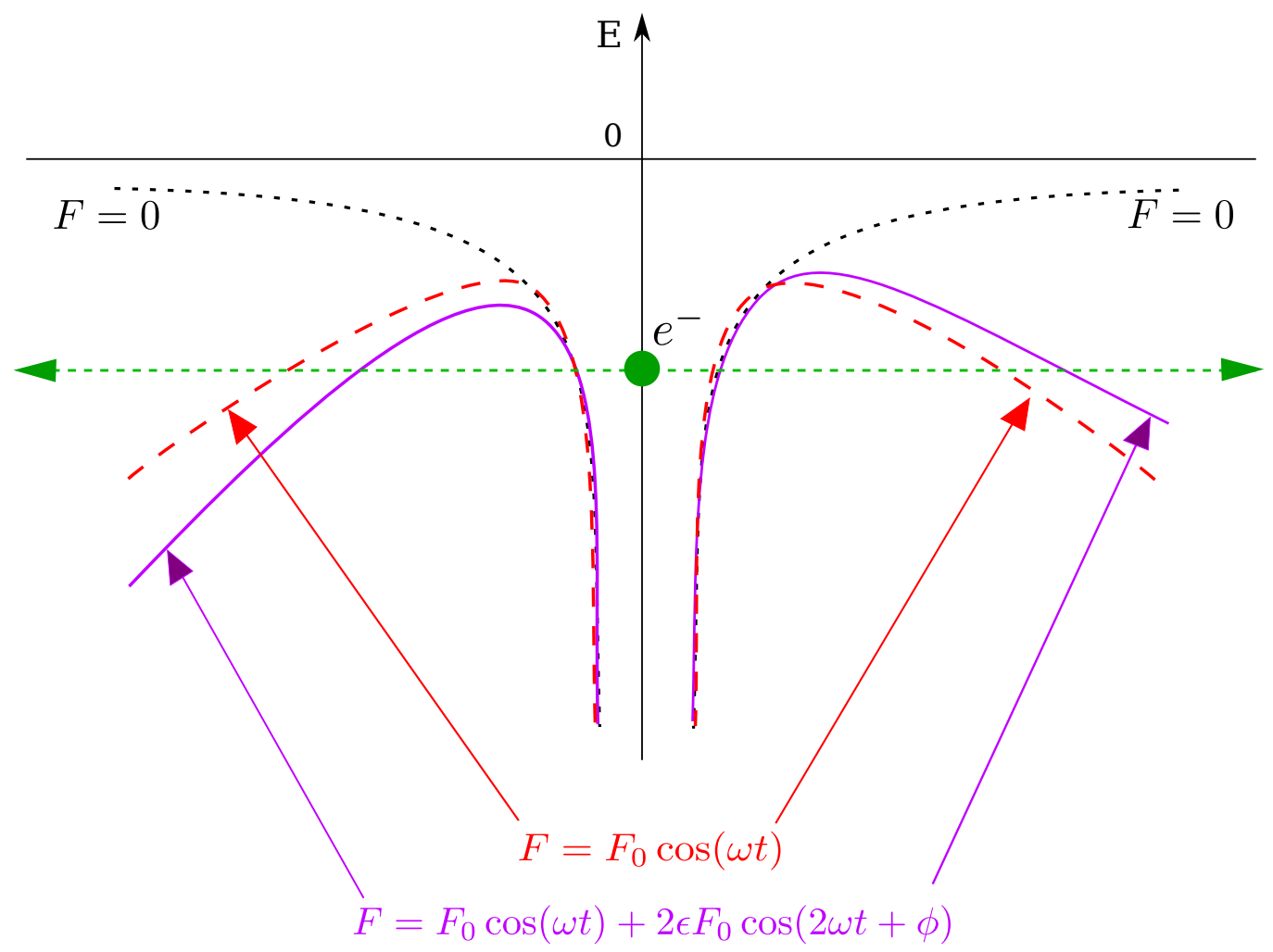

Figure 5.1: Schematic representation of the tunnelling barrier for two consecutive half-cycles. Under the influence of the SH field, the symmetry between two half-cycles is broken. Black dotted curve depicts the potential barrier without applied laser field. Red dotted curve is the potential barrier for both half-cycles for the case of interaction with one-color field. Magenta curve shows the asymmetrical bending of the tunnelling barrier for two half-cycles for the two-color field in parallel configuration

How do we obtain analytical expression for the phase between interferometer paths? The phase $S\left(t_{i}, t_{r}, p, \phi\right)$ (expression 3.27) can be written in perturbative approach for small $\epsilon$ as $S\left(t_{i}, t_{r}, p, \phi\right)=S_{0}\left(t_{i}, t_{r}, p\right)+\epsilon \sigma\left(t_{i}, t_{r}, p, \phi\right)$. It was shown [154], that in this case the dependence of relative intensities of even and odd harmonics on the phase $\sigma$ can be expressed as follows:

$$
I_{N}=I_{0}^{(N)} \begin{cases}|\cos (\epsilon \sigma(\phi, N))|^{2}, & \text { if } N \text { odd } \\ |\sin (\epsilon \sigma(\phi, N))|^{2}, & \text { if } N \text { even }\end{cases}
$$


where $\epsilon$ is the amplitude ratio between fundamental field and second harmonic, $N$ - harmonic number, $\phi$ - two-color phase. In the expression $5.5 I_{0}^{(N)}$ is the total fringe intensity, assumed to be the same for the neighboring even and odd harmonics.

Expression of the phase $S\left(t_{i}, t_{r}, p, \phi\right)$ was already analyzed in the Thesis in the chapter 4.2 (equation 4.33). We can further expand the expression 4.33, by substituting the vector potential $A(\tau)=-A_{0} \sin (\tau)-\epsilon A_{0} \sin (2 \tau+\phi)$ into 4.33. After regrouping the terms with obtain the expression for the phase:

$$
\begin{gathered}
S\left(\varphi_{i}, \varphi_{r}, k, \phi\right)=\frac{A_{0}^{2}}{2 \omega} \int_{\varphi_{i}}^{\varphi_{r}} d \tau\left(k+\frac{A(\tau)}{A_{0}}\right)^{2}+\frac{I_{p}}{\omega}\left(\varphi_{r}-\varphi_{i}\right)= \\
=\frac{A_{0}^{2}}{2 \omega} \int_{\varphi_{i}}^{\varphi_{r}} d \tau(k-\sin (\tau))^{2}+\frac{I_{p}}{\omega}\left(\varphi_{r}-\varphi_{i}\right)- \\
-\epsilon \frac{A_{0}^{2}}{\omega} \int_{\varphi_{i}}^{\varphi_{r}} d \tau \sin (2 \tau+\phi)(k-\sin (\tau))+\epsilon^{2} \frac{A_{0}^{2}}{2 \omega} \int_{\varphi_{i}}^{\varphi_{r}} d \tau \sin ^{2}(2 \tau+\phi)
\end{gathered}
$$

The second term in the obtained formula 5.6 corresponds to the first term in the perturbative expansion and gives the expression for the phase $\sigma$ :

$$
\sigma\left(\varphi_{i}, \varphi_{r}, k, \phi\right)=\frac{A_{0}^{2}}{\omega} \int_{\varphi_{i}}^{\varphi_{r}} d \tau \sin (2 \tau+\phi)(k-\sin (\tau))
$$

Detailed analysis of the perturbative expansion 5.6 is given in [154]. It shows, that term corresponding to the second order of $\epsilon$ can be neglected for the values of $\epsilon$ relevant for the experiment.

Real and imaginary parts of the complex phase $\sigma$ are extracted from experiment by the calculating the sum $I_{\text {sum }}^{(N)}$ and the difference $I_{\text {diff }}^{(N)}$ of the adjacent even and odd harmonics intensities:

$$
\begin{aligned}
& \operatorname{Re}(\sigma(N, \phi))=\frac{1}{2} \cos ^{-1}\left(\frac{I_{\text {diff }}^{(N)}(\phi)}{I_{0}^{(N)}}\right), \\
& \operatorname{Im}(\sigma(N, \phi))=\frac{1}{2} \cosh ^{-1}\left(\frac{I_{\text {sum }}^{(N)}(\phi)}{I_{0}^{(N)}}\right) .
\end{aligned}
$$

Our simulation of the real and imaginary values of complex phase $\sigma$ confirms 
the validity of this approach and our result is in a very good agreement with the $\sigma$, retrieved from experiment. Figure 5.2 shows our calculation of the imaginary and real parts of the complex phase $\sigma(N, \phi)$ between interferometer paths depending on the harmonic number $N$ and two-color delay $\phi$.
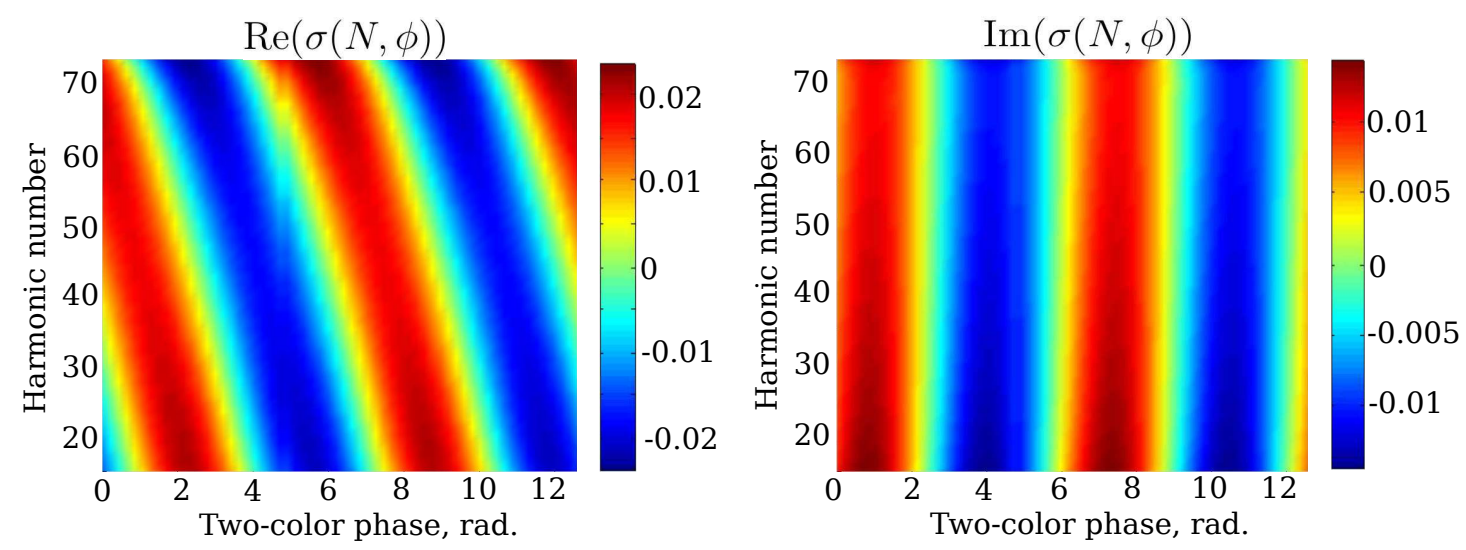

Figure 5.2: Complex phase $\sigma(N, \phi)$ between interferometer paths depending on the harmonic number $N$ and two-color delay $\phi$ : real part of the phase $\sigma$ is on the left plot, imaginary part - on the right plot. For comparison with experiment, please, see [154]. Parameters of the calculation: two-color HHG in He with fundamental field with wavelength $800 \mathrm{~nm}$ and intensity $I_{0}=4 \times 10^{14} \mathrm{~W} / \mathrm{cm}^{2}$, parameters of the SH: $\lambda_{S H}=400 \mathrm{~nm}, I_{S H}=0.01 I_{0}$.

Reconstruction procedure of the imaginary part of ionization time is based on the solving of system of equations, which describe the relation between values of $\operatorname{Re}(\sigma(N, \phi))$ and $\operatorname{Im}(\sigma(N, \phi))$ obtained in theory and retrieved from experiment. Extracted imaginary ionization time is in a good agreement with its SFA value [154].

\subsubsection{Multicolor scheme}

Here we describe the results of our work [162], where we have developed an extension of the reconstruction procedure, presented in [93]. This extension opens the possibility of extracting the imaginary ionization time. This possibility is achieved by introducing one more control parameter via the addition 
of one more perturbative field into the two-color scheme of HHG: the third harmonic of the fundamental field. The polarization of this field is perpendicular to that of the fundamental laser field, see Fig. 5.3. The phase between the second and the third harmonic $\phi_{m}$, or the multicolor phase, is the additional parameter that allows one to fine-tune the process of high harmonic generation in order to gain access to more variables.

The saddle point solutions for the multicolor field were analyzed in Section 4.3. The vector potential of the perturbative perpendicular field is described by the formula Eq.(4.34).

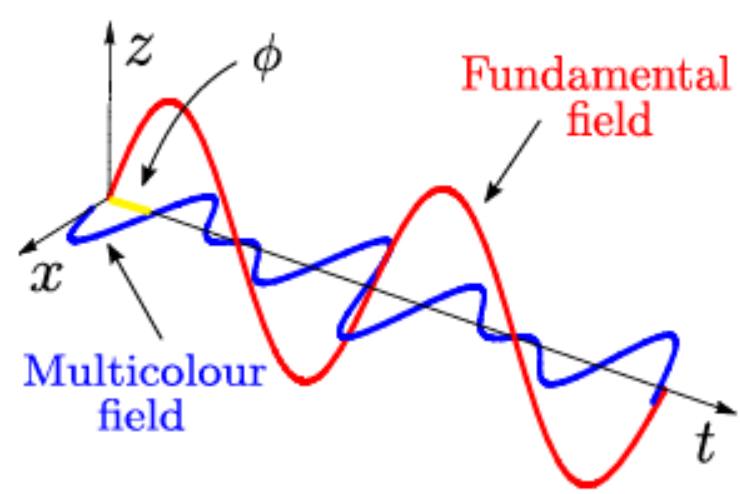

(a)

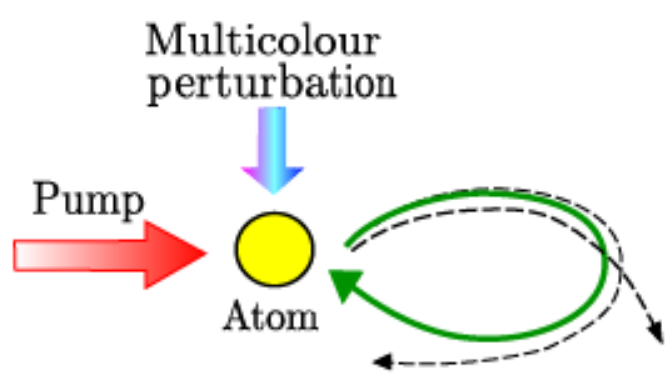

)

Figure 5.3: Schematic illustration of the multicolor field (on the left) and trajectories in multicolor field (on the right).

In order to understand how third harmonic (TH) field modifies the process, let us look at the calculation for two different values of the multicolor delay $\phi_{m}=0 \mathrm{rad}$ and $\phi_{m}=2.1 \mathrm{rad}$. These values of $\phi_{m}$ were chosen only for the purposes of illustration of the principle.

The modulation of the HHG signal due to the perturbative field is calculated for both of the chosen multicolor delays $\phi_{m}$ using the semi-classical approach (quantum gate) using the classical approach (classical gate). It is convenient to look at the results of these calculations for the position of the optimal phase $\phi_{\text {opt }}$ between the fundamental field and the second harmonic. The results were shown in Figure 4.24 for both multicolor delays. In the case $\phi_{m}=0$ rad the classical description coincides with the quantum gate for the optimal delays $\phi$. 
However, for $\phi_{m}=2.1 \mathrm{rad}$, the classical approach fails. This means that we have a chance to identify the non-classical part of the evolution.

Classical description of the electron return to the parent ion 4.45 assumes zero lateral velocity at the moment of ionization and neglects the tunnelling barrier. As a consequence, the parameters of electron trajectories are restricted to real values. If this approach does not work, it signifies the importance of the imaginary components of the trajectory parameters and the failure of classical assumptions.

Why does the classical gate work in one case and not in the other? Let us obtain expressions for both the quantum 5.28 and the classical gates.

The classical gate is defined as:

$$
G=\exp \left[-\frac{1}{2} v_{c l, \perp}^{2}\left(t_{i}\right) t_{i}^{\prime \prime}\right]
$$

The classical lateral velocity $v_{\perp}^{c l}=p_{\perp}^{c l}+A_{\perp}(t)$ is derived from the condition of zero lateral displacement of the electron between ionization and recombination times:

$$
\int_{t_{i}^{\prime}}^{t_{r}} v_{\perp}^{c l} d t=\int_{t_{i}^{\prime}}^{t_{r}}\left(p_{\perp}^{c l}+A_{\perp}(t)\right) d t=0
$$

Integrating the above expression, we obtain:

$$
p_{\perp}^{c l}=-\frac{1}{t_{r}-t_{i}^{\prime}} \int_{t_{i}^{\prime}}^{t_{r}} A_{\perp}(t) d t
$$

The expression for calculating the classical gate becomes:

$$
G=\exp \left[-\frac{t_{i}^{\prime \prime}}{2}\left(-\frac{1}{t_{r}-t_{i}^{\prime}} \int_{t_{i}^{\prime}}^{t_{r}} A_{\perp}\left(t_{i}\right) d t+A_{\perp}(t)\right)^{2}\right]
$$

The optimal phases $\phi_{o p t}^{c l}$ are the maxima of the classical gate. They are found as solutions to the equation:

$$
\left.\frac{\partial G}{\partial \phi}\right|_{\phi_{o p t}^{c l}}=0
$$


Differentiating the expression 5.13 with respect to the phase $\phi$, we obtain:

$$
\left(A_{\perp}\left(t_{i}\right)-\frac{1}{t_{r}-t_{i}^{\prime}} \int_{t_{i}^{\prime}}^{t_{r}} A_{\perp}\left(t_{i}\right) d t\right)\left(\frac{\partial A_{\perp}(t)}{\partial \phi}-\frac{1}{t_{r}-t_{i}^{\prime}} \frac{\partial}{\partial \phi} \int_{t_{i}^{\prime}}^{t_{r}} A_{\perp}(t) d t\right)=0
$$

If we remember, that $\left(A_{\perp}\left(t_{i}\right)-\frac{1}{t_{r}-t_{i}} \int_{t_{i}}^{t_{r}} A_{\perp}(t) d t\right)=v_{\perp}^{c l}\left(t_{i}\right)$, then we see that the classical gate has maximum at such delays $\phi_{o p t}^{c l}$, at which the initial lateral velocity of the electron is zero:

$$
v_{\perp}^{c l}\left(t_{i}, \phi_{o p t}^{c l}\right)=p_{\perp}^{c l}+A_{\perp}\left(t_{i}\right)=0
$$

This condition is satisfied when the vector potential at the moment of ionization is close to zero $A_{\perp}\left(t_{i}\right) \approx 0$, which means zero lateral drift. Thus, the delays, at which the perpendicular vector potential at the moment of ionization is zero, are always close to the optimal delays for the classical gate $\phi_{o p t}^{c l}$.

The expression for the classical gate in case of the multicolor field 4.34 is:

$$
\begin{aligned}
G\left(t_{i}, p_{\perp}, \phi, \phi_{m}\right) & =\exp \left[-\frac{1}{2} v_{\perp}^{c l}\left(t_{i}\right)^{2} t_{i}^{\prime \prime}\right]= \\
& =\exp \left[-\frac{1}{2} p_{\perp, c l}^{2} t_{i}^{\prime \prime}+p_{\perp}^{c l} t_{i}^{\prime \prime} \epsilon_{2} A_{0} \sin \left(2 \omega t_{i}+\phi\right)+\right. \\
& +p_{\perp}^{c l} t_{i}^{\prime \prime} \epsilon_{3} A_{0} \sin \left(3 \omega t_{i}+\phi+\phi_{m}\right)- \\
& -\frac{\epsilon_{2}^{2} A_{0}^{2}}{2} \sin ^{2}\left(2 \omega t_{i}+\phi\right)-\frac{\epsilon_{3}^{2} A_{0}^{2}}{2} \sin ^{2}\left(3 \omega t_{i}+\phi+\phi_{m}\right)+ \\
& \left.-\epsilon_{2} \epsilon_{3} \sin \left(2 \omega t_{i}+\phi\right) \sin \left(3 \omega t_{i}+\phi+\phi_{m}\right)\right]
\end{aligned}
$$

As we saw, at optimal delays $A_{\perp}\left(t_{i}\right) \approx 0$ and the expression for the classical gate 5.13 becomes:

$$
G\left(\phi_{\text {opt }}\right) \approx \exp \left[-\frac{1}{2} t_{i}^{\prime \prime} p_{\perp, c l}^{2}\right]
$$

Let us now derive the expression for the quantum gate and compare it to the classical gate. The quantum gate is defined as the exponential factor responsible for the amplitude modulation of the harmonic dipole:

$$
Q=\exp \left[2 \operatorname{Im} S\left(t_{i}, t_{r}, p_{\perp}, \phi, \phi_{m}\right)\right]
$$


The expression for the multicolor phase $S\left(t_{i}, t_{r}, p_{\perp}, \phi, \phi_{m}\right)$ is:

$$
\begin{gathered}
S\left(t_{i}, t_{r}, p_{\|}, p_{\perp}, \phi, \phi_{m}\right)=\frac{1}{2} \int_{t_{i}}^{t_{r}} d t\left(p_{\|}+A_{p a r}(\tau)\right)^{2}+\frac{1}{2} \int_{t_{i}}^{t_{r}} d t\left(p_{\perp}+A_{p e r}\right)^{2}+ \\
+I_{p}\left(t_{r}-t_{i}\right)
\end{gathered}
$$

We will consider further only the terms in the phase 5.20, that depend on the phase delay $\phi$ and the multicolor phase $\phi_{m}$.

Integration in the expression 5.20 is performed along the contour shown in Figure 3.1: first along the imaginary axis, then along the real axis, schematically written as:

$$
S=i \int_{t_{i}^{\prime \prime}}^{0}+\int_{t_{i}^{\prime}}^{t_{r}}=\operatorname{Re}\left(i \int_{t_{i}^{\prime \prime}}^{0}\right)+i \operatorname{Im}\left(i \int_{t_{i}^{\prime \prime}}^{0}\right)+\operatorname{Re}\left(\int_{t_{i}^{\prime}}^{t_{r}}\right)+i \operatorname{Im}\left(\int_{t_{i}^{\prime}}^{t_{r}}\right)
$$

Note, that at the moment we do not yet consider ionization and recombination times and the momentum in the expression 5.20 and 5.21 as saddle points. The imaginary part of the phase 5.20 is calculated as:

$$
\begin{aligned}
& \operatorname{Im} S\left(t_{i}, t_{r}, p_{\perp}, \phi, \phi_{m}\right)=\operatorname{Re} \frac{1}{2} \int_{t_{i}^{\prime \prime}}^{0}\left(p_{\perp}+A_{\perp}\left(t_{i}^{\prime}+i \tau\right)\right)^{2} d \tau-I_{p} t_{i}^{\prime \prime}= \\
= & -\frac{1}{2} p_{\perp} t_{i}^{\prime \prime}+p_{\perp} \operatorname{Re} \int_{t_{i}^{\prime \prime}}^{0} A_{\perp}\left(t_{i}^{\prime}+i \tau\right) d \tau+\frac{1}{2} \operatorname{Re} \int_{t_{0}^{\prime \prime}}^{0} A_{\perp}^{2}\left(t_{i}^{\prime}+i \tau\right) d \tau-I_{p} t_{i}^{\prime \prime}(5
\end{aligned}
$$

Let us now introduce the parameter $p_{\text {opt }}$, the optimal tunneling momentum. It is such a lateral momentum, at which the change in the ionization rate due to the perturbative fields is maximized.

The ionization amplitude is defined by the imaginary part of the phase 5.20 , when integration is performed only along the imaginary axis; the time $t_{i}^{\prime}$ is the time of exit from the tunneling barrier in our model. The optimal momentum is found as a solution of the equation:

$$
\left.\frac{\partial \operatorname{Im} S}{\partial p_{\perp}}\right|_{p_{\mathrm{opt}}}=0
$$


We see that the condition on the optimal momentum coincides with requiring zero imaginary displacement during the tunnelling step:

$$
\operatorname{Re} \int_{t_{i}^{\prime \prime}}^{0}\left(p_{o p t}+A_{\perp}\left(t_{i}^{\prime}+i \tau\right)\right) d \tau=0
$$

Thus, the optimal tunneling momentum is:

$$
\begin{gathered}
p_{\mathrm{opt}}=\frac{1}{t_{i}^{\prime \prime}} \operatorname{Re} \int_{t_{i}^{\prime \prime}}^{0} A_{\perp}\left(t_{i}^{\prime}+i \tau\right) d \tau= \\
=-\frac{\epsilon_{2} A_{0}}{2 t_{i}^{\prime \prime}} \sin \left(2 \omega t_{i}^{\prime}+\phi\right) \sinh 2 \omega t_{i}^{\prime \prime}-\frac{\epsilon_{3} A_{0}}{3 t_{i}^{\prime \prime}} \sin \left(3 \omega t_{i}^{\prime}+\phi+\phi_{m}\right) \sinh 3 \omega t_{i}^{\prime \prime}
\end{gathered}
$$

We can rewrite the expression for the imaginary part of the phase 5.20 as:

$$
\operatorname{Im} S=-\frac{1}{2} p_{\perp}^{2} t_{i}^{\prime \prime}+p_{\perp} p_{\mathrm{opt}} t_{i}^{\prime \prime}+\frac{1}{2} \operatorname{Re} \int_{t_{0}^{\prime \prime}}^{0} A_{\perp}^{2}\left(t_{i}^{\prime}+i \tau\right) d \tau-I_{p} t_{i}^{\prime \prime}
$$

After the remaining integration in the above expression is performed, we obtain the quantum gate:

$$
\begin{gathered}
Q\left(t_{i}, t_{r}, p_{\perp}, \phi, \phi_{m}\right)=\exp \left\{-\frac{1}{2} p_{\perp}^{2} t_{i}^{\prime \prime}+p_{\perp} p_{\mathrm{opt}} t_{i}^{\prime \prime}-\right. \\
-\frac{\epsilon_{1}^{2} A_{0}^{2}}{2} \sin ^{2}\left(2 \omega t_{i}^{\prime}+\phi\right) \frac{\sinh 4 \omega t_{i}^{\prime \prime}}{4 \omega}-\frac{\epsilon_{2}^{2} A_{0}^{2}}{2} \sin ^{2}\left(3 \omega t_{i}^{\prime}+\phi+\phi_{m}\right) \frac{\sinh 6 \omega t_{i}^{\prime \prime}}{6 \omega}+ \\
+\frac{\epsilon_{1} \epsilon_{2} A_{0}^{2}}{2}\left[\cos \left(2 \omega t_{i}^{\prime}+\phi\right) \cos \left(3 \omega t_{i}^{\prime}+\phi+\phi_{m}\right)\left(\frac{\sinh 5 \omega t_{i}^{\prime \prime}}{5 \omega}-\frac{\sinh \omega t_{i}^{\prime \prime}}{\omega}\right)\right. \\
\left.\left.-\sin \left(2 \omega t_{i}^{\prime}+\phi\right) \sin \left(3 \omega t_{i}^{\prime}+\phi+\phi_{m}\right)\left(\frac{\sinh 5 \omega t_{i}^{\prime \prime}}{5 \omega}+\frac{\sinh \omega t_{i}^{\prime \prime}}{\omega}\right)\right]\right\}
\end{gathered}
$$

We see that the primary difference between the expressions for the quantum and classical gates is the presence of the hyperbolic functions in the expression for the quantum gate 5.28. These hyperbolic functions depend on the imaginary part of the ionization time, i.e. these are exactly the parts we are interested in. In which situations do these factors become important?

Let us look closely at the figure 4.24. First, we will examine the case of $\phi_{m}=0$ 
rad. The vector potentials of both the second and the third harmonic at the moment of ionization for the optimal delays are close to zero, as well as the overall vector potential. It means that the electron at the exit of the barrier does not obtain any lateral drift from the perturbative field. Recalling the expression for the vector potential at the moment of ionization, the above conditions are written as:

$$
\begin{gathered}
A_{\perp}\left(t_{i}\right)=-\epsilon_{2} A_{0} \sin \left(2 \omega t_{i}+\phi_{0}\right)-\epsilon_{3} A_{0} \sin \left(3 \omega t_{i}+\phi_{0}+\phi_{m}\right) \approx 0, \\
A_{2 \omega}\left(t_{i}\right)=-\epsilon_{2} A_{0} \sin \left(2 \omega t_{i}+\phi_{0}\right) \approx 0, \\
A_{3 \omega}\left(t_{i}\right)=-\epsilon_{3} A_{0} \sin \left(3 \omega t_{i}+\phi_{0}+\phi_{m}\right) \approx 0
\end{gathered}
$$

When all conditions above are approximately satisfied, as indeed is possible for $\phi_{m}=0$, the optimal lateral momentum is also close to zero, $p_{\text {opt }}=0$ and the terms in the expression for the quantum gate 5.28 that contain sin functions become negligible. Then we can re-rewrite the gate as:

$$
\begin{gathered}
Q\left(t_{i}, t_{r}, p_{\perp}, \phi_{0}, \phi_{m}\right) \approx \exp \left\{-\frac{1}{2} p_{\perp}^{2} t_{i}^{\prime \prime}-\right. \\
\left.+\frac{\epsilon_{1} \epsilon_{2} A_{0}^{2}}{2} \cos \left(2 \omega t_{i}^{\prime}+\phi_{0}\right) \cos \left(3 \omega t_{i}^{\prime}+\phi_{0}+\phi_{m}\right)\left(\frac{\sinh 5 \omega t_{i}^{\prime \prime}}{5 \omega}-\frac{\sinh \omega t_{i}^{\prime \prime}}{\omega}\right)\right\}
\end{gathered}
$$

If the sin functions are close to zeros, the cos functions in 5.30 are close to unity. However, they do not introduce dependence on the phases $\phi_{0}$ and $\phi_{m}$ and contribute only to the uniform background. Thus, at optimal delays $\phi_{0}$, the approximate expression for the quantum gate becomes very similar to the classical gate:

$$
Q\left(\phi_{0}\right) \approx \exp \left\{-\frac{1}{2} p_{\perp}^{2} t_{i}^{\prime \prime}\right\}
$$

The approximate expressions for the classical 5.18 and quantum 5.31 gates would have matched at optimal delays (for the case $\phi_{m}=0$ ), had it not been for the fact that the classical canonical momentum $p_{\perp}^{c l}$ and the quantum saddle point for the canonical momentum are not equal. What is difference between them?

Both $p_{\perp}$ and $p_{\perp}^{c l}$ are obtained form the conditions on the zero lateral displacement. In the classical case, the electron starts at the origin (no tunneling barrier), and returns exactly to the same point. In the quantum case, the 
equation for the return takes into account the electron displacement after ionization.

Let us write down both equations for the electron return. We start with the quantum expression

$$
\begin{aligned}
\int_{t_{i}^{\prime \prime}}^{0}\left(p_{\perp}+A_{\perp}(\tau)\right) d \tau+\int_{t_{i}^{\prime}}^{t_{r}}\left(p_{\perp}+A_{\perp}(\tau)\right) d \tau & =0 \\
x_{\text {exit }}+\int_{t_{i}^{\prime}}^{t_{r}}\left(p_{\perp}+A_{\perp}(\tau)\right) d \tau & =0 \\
x_{\text {exit }}+p_{\perp}\left(t_{r}-t_{i}^{\prime}\right)+\int_{t_{i}^{\prime}}^{t_{r}} A_{\perp}(\tau) d \tau & =0
\end{aligned}
$$

We denote $\Delta t=t_{r}-t_{i}^{\prime}$ and $\Delta x_{c l}=\int_{t_{i}^{\prime}}^{t_{r}} A_{\perp}(\tau) d \tau$ and rewrite the last equation:

$$
x_{e x i t}+p_{\perp} \Delta t+\Delta r_{c l}=0
$$

The classical return condition 5.11 yields similar expression:

$$
\begin{aligned}
\int_{t_{i}}^{t_{r}}\left(p_{\perp}^{c l}+A_{\perp}(\tau)\right) d \tau & =0 \\
p_{\perp}^{c l} \Delta t+\int_{t_{i}}^{t_{r}} A_{\perp}(\tau) d \tau & =0 \\
p_{\perp}^{c l} \Delta t+\Delta r_{c l} & =0
\end{aligned}
$$

If we compare the expressions Eq.(5.33) and Eq.(5.34), we find the connection between the classical and the quantum canonical momenta:

$$
p_{\perp}=p_{\perp}^{c l}-\frac{x_{e x i t}}{\Delta t}
$$

The lateral displacement is easily calculated:

$x_{e x i t}=\frac{\epsilon_{2} A_{0}}{2 \omega} \cos \left(2 \omega t_{i}+\phi\right)\left(1-\cosh 2 \omega t_{i}^{\prime \prime}\right)+\frac{\epsilon_{3} A_{0}}{3 \omega} \cos \left(3 \omega t_{i}+\phi+\phi_{m}\right)\left(1-\cosh 3 \omega t_{i}^{\prime \prime}\right)$ 
Under the conditions that we consider, when the vector potentials of the second and the third harmonics at the moment of ionization are close to zero $\left(\sin \left(2 \omega t_{i}+\phi_{0}\right) \approx 0\right.$ and $\left.\sin \left(3 \omega t_{i}+\phi_{0}+\phi_{m}\right) \approx 0\right)$, the lateral displacement after the ionization stops to depend on the phase $\phi$, because the cos functions are close to unity and the value of the displacement stays constant for all harmonic numbers. Thus, we can rewrite the connection between the classical and quantum (saddle point) momenta as:

$$
p_{\perp}=p_{\perp}^{c l}+\text { const }
$$

It means, that quantum and classical gates at optimal delays $\phi_{0}$ are indeed equal, up to a constant background:

$$
G\left(\phi_{0}\right) \approx Q\left(\phi_{0}\right)
$$

Having analyzed the case of $\phi_{m}=0$, it is now easy to understand why the classical gate failed when $\phi_{m}=2.1 \mathrm{rad}$. The delays $\phi$, at which the vector potentials of the second and third harmonics are close to zero at the ionization instant are far from the calculated optimal delays. It means, that the vector potentials of the $\mathrm{SH}$ and $\mathrm{TH}$ fields are not close to zero at the ionization times for the optimal delays, while, as we see from the figure 4.24, the overall perpendicular vector potential is close to zero. Nothing changes for the classical gate. It still has maximum near the zeros of the overall vector potential. However, since these zeros are no longer close to the individual zeros of the $\mathrm{SH}$ and $\mathrm{TH}$ vector potentials, the terms with the hyperbolic functions in the expression 5.28 become important, and the quantum gate becomes different from its classical counterpart.

\section{Reconstruction procedure}

Previously, we have shown how we can control the importance of the imaginary components of the saddle point solutions in the multicolor regime of HHG. With changing only the multicolor delay $\phi_{m}$, we can turn on and off the effects of tunneling in the position of the optimal delay $\phi_{0}$. We will now show how this 
property of the multicolor scheme may be used in the reconstruction procedure of imaginary and real parts of the ionization time. The analysis follows our work [162].

The recombination times $t_{r}$ can be measured in the experiment with the RABBIT technique [163] or by measuring the relative intensities of even and odd harmonics, as was described in [93]. In our procedure we assume that the recombination times are known or have been reconstructed, e.g. using the procedure developed in [93].

The variables left for the reconstruction are real $t_{i}^{\prime}$ and imaginary $t_{i}^{\prime \prime}$ parts of the ionization time. The imaginary ionization time cannot be extracted unless the 'degeneracy' between the quantum and the classical gates is lifted. It is done by controlling the multicolor phase $\phi_{m}$.

In order to extract the two parameters from the experimental data, we need two independent values of the optimal delay $\phi_{0}$ and, consequently, two equations. It can be achieved by measuring the multicolor HHG spectrum twice for different multicolor delays $\phi_{m}$.

The equations for each harmonic number $N$, that give the position of the maximum of the quantum gates for these two measurements, are:

$$
\begin{aligned}
& \left.\frac{\partial Q\left(t_{r N}, t_{i N}, \phi, \phi_{m}^{1}\right)}{\partial \phi}\right|_{\phi_{1}^{N}}=0, \\
& \left.\frac{\partial Q\left(t_{r N}, t_{i N}, \phi, \phi_{m}^{2}\right)}{\partial \phi}\right|_{\phi_{2}^{N}}=0 .
\end{aligned}
$$

where index $N$ denotes the harmonic number. Solving these equations together gives us the values of the real and imaginary ionization times, given that the positions of the optimal delays $\phi_{1}$ and $\phi_{2}$ are measured in the experiment.

In order to illustrate the reconstruction procedure, we simulate the modulation of the harmonic spectrum. For simplicity, we present our results as a dependence on the recombination time instead of the harmonic number, keeping in mind, that the signals in the time-domain and the frequency domain are connected by the Fourier transform, and that there is a well-defined relationship 
between the recombination time and the emitted harmonic order. We calculate the harmonic spectrum for the two different multicolor delays $\phi_{m}=1.5413 \mathrm{rad}$ and $\phi_{m}=4.5871 \mathrm{rad}$, that were chosen randomly by the built-in MATLAB function rand. The results are shown in Figure 5.4.
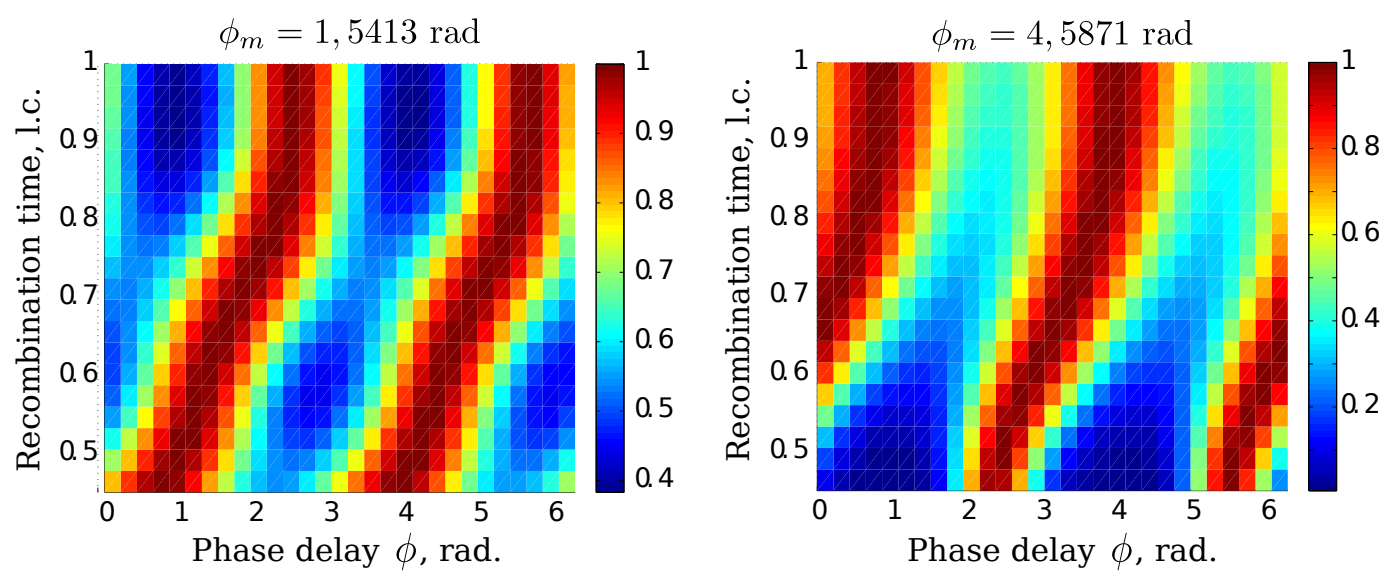

Figure 5.4: Normalized HHG spectrum for two values of multicolor delays: $\phi_{m}=1,5413 \mathrm{rad}$ on the left, $\phi_{m}=4,5871 \mathrm{rad}$ on the right. Parameters of the driving pulse: $I_{0}=10^{14} \mathrm{~W} / \mathrm{cm}^{2}, \lambda=1600 \mathrm{~nm}$, parameters of the perturbation: $\lambda_{S H}=800 \mathrm{~nm}, I_{S H}=0.02 I_{0}$, $\lambda_{T H}=400 \mathrm{~nm}, I_{T H}=0.01 I_{0}$.

Extracting the optimal phases $\phi_{1}^{N}$ and $\phi_{2}^{N}$ from both spectra, we plug them in into the equations 5.39 and 5.40, assuming that we already know the recombination times $t_{r N}$. We obtain the two equations for the two variables: the real and the imaginary parts of the ionization time $t_{i N}$, and we solve those together, for each harmonic number $N$. The results are shown on the figure 5.5 , together with the quantum saddle point solutions for comparison. 


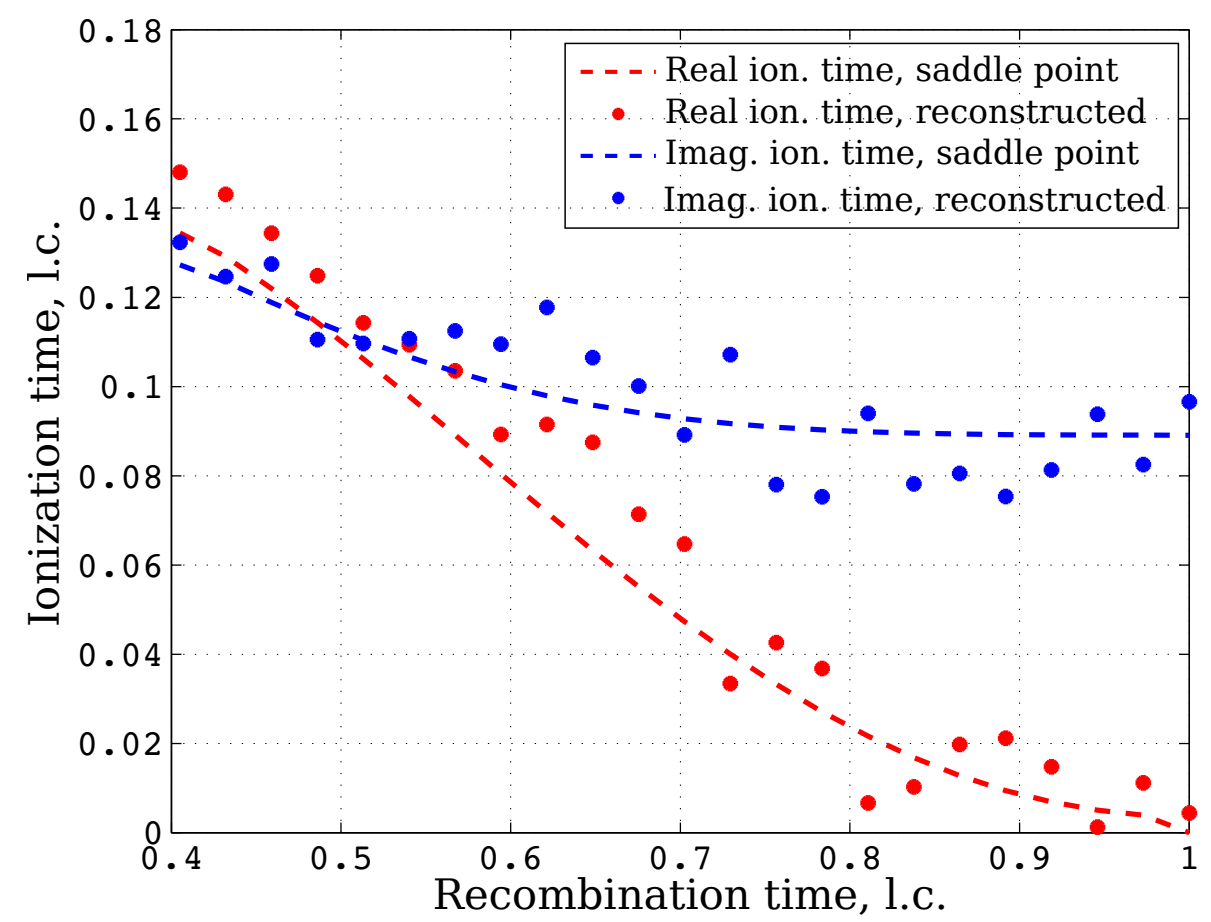

Figure 5.5: Reconstructed real and imaginary ionization times from the simulated multicolor HHG spectra with multicolor delays $\phi_{m}=1,5413$ and $\phi_{m}=4,5871$. Parameters of the driving pulse: $I_{0}=10^{14}$ $\mathrm{W} / \mathrm{cm}^{2}, \lambda=1600 \mathrm{~nm}$, parameters of the perturbation: $\lambda_{S H}=800$ $\mathrm{nm}, I_{S H}=0.02 I_{0}, \lambda_{T H}=400 \mathrm{~nm}, I_{T H}=0.01 I_{0}$.

The reconstruction procedure can be simplified if we remember that our analysis showed the existence of such multicolor delays $\phi_{m}^{1}$, at which the classical description is applicable. Suppose we first use the multicolor delay which restores the degeneracy between the quantum and the classical gates. Then we can measure the two-dimensional harmonic spectrum and extract the real part of ionization time, using only one equation 5.39. After the real part of the ionization time is obtained, only one parameter is left for the reconstruction- the imaginary ionization time. Recording the 2D harmonic spectrum for a different value of the multicolor phase $\phi_{m}^{2}$, that demands the use of the quantum gate for its description, we extract the new value of the optimal phase $\phi_{2}$, for each harmonic number. Plugging them into the equation 5.40, we solve it and obtain the imaginary ionisation time. The results of this simplified procedure is shown on the figure 5.6. The values of the multicolor phases are $\phi_{m}^{1}=0 \mathrm{rad}$ 
and $\phi_{m}^{2}=2.1 \mathrm{rad}$, used previously in our analysis.

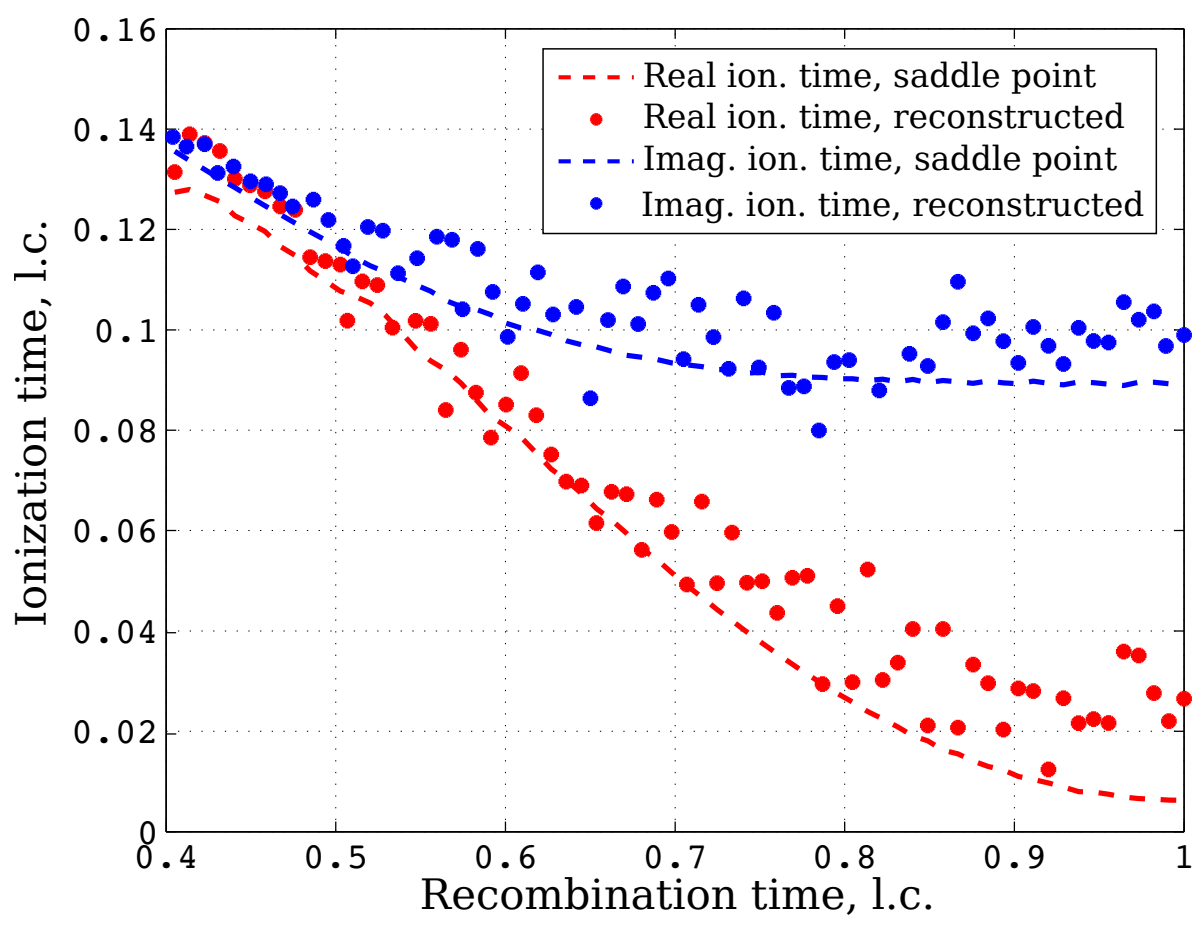

Figure 5.6: Reconstructed real and imaginary ionization times from the simulated multicolor HHG spectra with multicolor delays $\phi_{m}=0$ and $\phi_{m}=2,1$. Parameters of the driving pulse: $I_{0}=10^{14} \mathrm{~W} / \mathrm{cm}^{2}$, $\lambda=1600 \mathrm{~nm}$, parameters of the perturbation: $\lambda_{S H}=800 \mathrm{~nm}$, $I_{S H}=0.02 I_{0}, \lambda_{T H}=400 \mathrm{~nm}, I_{T H}=0.01 I_{0}$. 


\section{Outlook: dynamics in $\mathrm{CO}_{2}$}

One of the key objective of high harmonic spectroscopy is in revealing molecular dynamics with attosecond and angstrom resolution, with the dream of imaging the process of bond breaking and bond making during chemical reactions. This bold goal poses a lot of challenges, due to complexity of the dynamics and the entanglement between spatial and temporal information in the observables. The multidimensional high harmonic spectroscopy we are introducing in this Thesis is a way of disentangling spatial and temporal information encoded in the harmonic spectra, and is a new tool for attosecond physics. In this chapter, we present an outlook on how this new tool may be used by looking the example of a carbon dioxide molecule. Initial analysis, performed in the Thesis and presented in this chapter, was used in work [164], where two-dimensional HHG experiments in $\mathrm{CO}_{2}$ were studied and ionization dynamics was resolved.

The $\mathrm{CO}_{2}$ molecule is a complex system with many electrons, for which multielectron effects have to be taken into account. The applied strong laser field triggers the dynamics of multiple electrons during and after ionization. The signatures of this dynamics appear in the HHG spectrum, however, different effects are mixed together. Multidimensional high harmonic spectroscopy can be used to differentiate structural and dynamical features in the HHG spectrum, leading to more insight into the molecular dynamics upon ionization and recombination [164].

An important step in understanding the dynamics in the ion is our ability, afforded by the HHG process, to splitting the analysis of the process in steps, in order to distinguish their separate roles or to study their interplay. Calculation implemented in [164] allows to model the process of high harmonic generation 
in a molecule step by step and investigate the contributions of ionization, electron propagation in the continuum, the dynamics in the ion between ionization and recombination, and the recombination step.

The calculation is based on the factorization of the harmonic dipole in the time domain, with the subsequent Fourier transform yielding the harmonic spectrum. The expression for the factorized harmonic dipole in time domain was obtained in Section 3.3.3. Here we re-write the formula for one channel of the HHG process:

$$
\mathbf{D}_{m n}\left(t_{r}\right)=a_{i o n}^{n}\left(\mathbf{p}, t_{i}\right) a_{\text {prop }}^{m n}\left(\mathbf{p}, t_{r}, t_{i}\right) a^{m n}\left(\mathbf{p}, t_{r}, t_{i}\right) a_{r e c}^{m}\left(\mathbf{p}, t_{r}\right)
$$

where $m$ and $n$ denote the recombination and the ionization states in the ion, respectively. We calculate the ionization $a_{i o n}^{n}\left(\mathbf{p}, t_{i}\right)$, recombination $a_{r e c}^{m}\left(\mathbf{p}, t_{r}\right)$, propagation $a_{\text {prop }}^{m n}\left(\mathbf{p}, t_{r}, t_{i}\right)$ amplitudes and the dynamics in the ion $a^{m n}\left(\mathbf{p}, t_{r}, t_{i}\right)$ separately. Each amplitude may, in principle, be described with different levels of approximation adequate for the required accuracy. The effect of each of the steps, and each of the pathways $n \rightarrow m$ can be evaluated by changing the weight of its contribution to the total harmonic dipole and thus the total harmonic spectrum, from turning it off completely to studying only its role.

The $\mathrm{CO}_{2}$ molecule presents a very instructive case for the analysis of the underlying dynamics. It is a linear molecule that belongs to the symmetry group $\mathrm{D}_{\infty h}$. As a consequence of its symmetry, it has no permanent dipole. For the description of the experiments discussed in this thesis, only four ionic states and only four of its highest molecular orbitals are important.

The figure 6.1 shows the energy structure of the relevant electronic states of the $\mathrm{CO}_{2}$ ion and the shapes of the associated Dyson orbitals. All of these ionic states are well approximated as single-hole states, thus it is possible to think of ionization as removal of the electron from the corresponding Dyson orbital. 

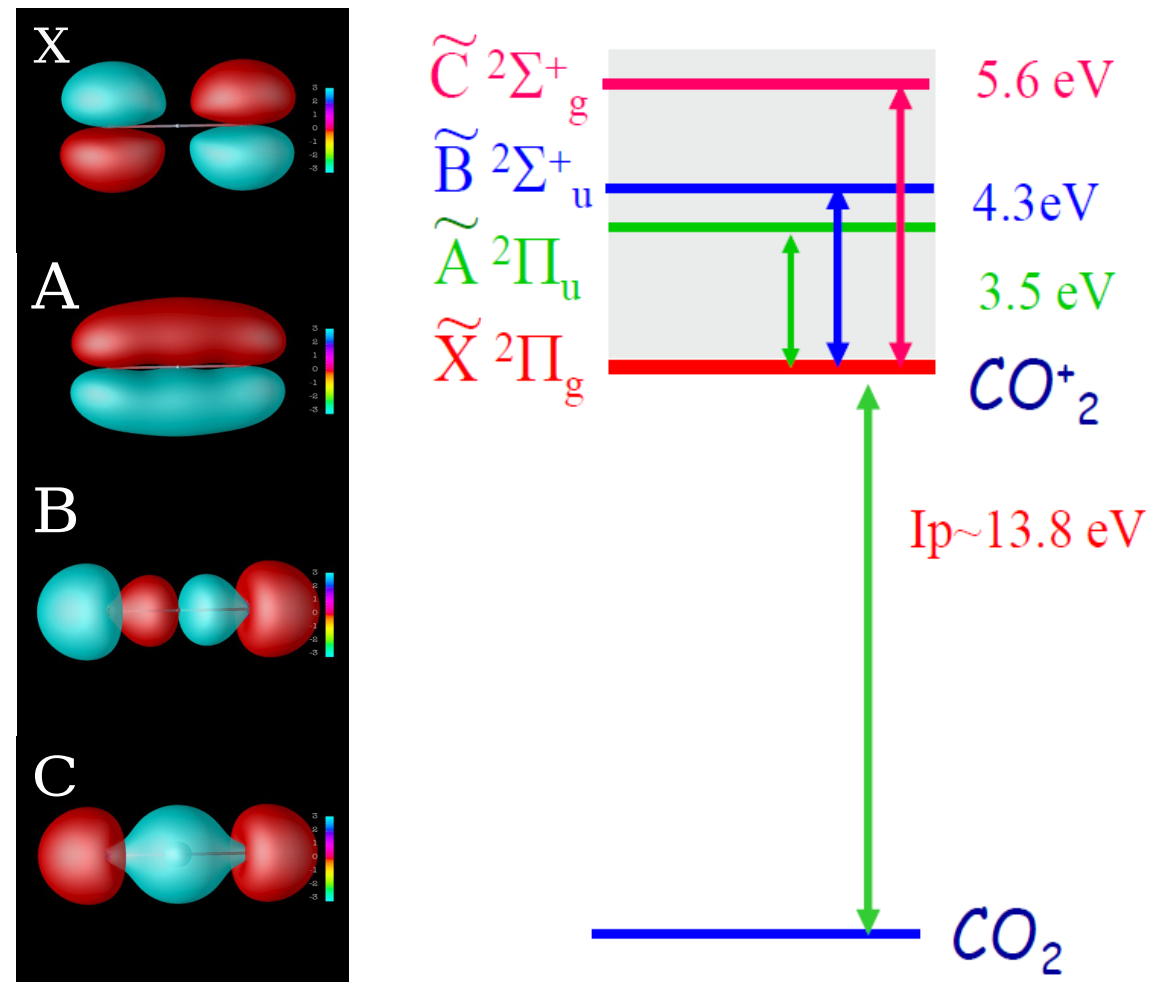

Figure 6.1: On the left - four key Dyson orbitals of the $\mathrm{CO}_{2}$ ion: $\mathrm{X}, \mathrm{A}, \mathrm{B}$, and $\mathrm{C}$, that correspond to the ionization from HOMO, HOMO-1, HOMO-2 and HOMO-3, respectively. On the right - energy level structure of the $\mathrm{CO}_{2}$ ion. Dyson orbitals were obtained in the work [127] by S. Patchkovskii.

Ionization may start from any of the four Dyson molecular orbitals. For convenience we denote the ionic levels with letters X, A, B and C. Each ionic level corresponds to the ionization from one of the molecular orbitals: the ground ion state $\mathrm{X}$ - to the ionization from HOMO, the first excited state - to the ionization from HOMO-1, the second excited state B - to the ionization from HOMO-2, the third excited ion state $\mathrm{C}$ - to the ionization from HOMO-3. Due to the laser induced dynamics in the ion after ionization, the hole may move between the orbitals and the electron may recombine to any of the three excited states of the ion or to the ground state. Thus, 16 channels in the process should be considered: XX, XA, XB, XC, AX, AA, AB, AC, BX, BA, BB, $\mathrm{BC}, \mathrm{CX}, \mathrm{CA}, \mathrm{CB}$ and $\mathrm{CC}$. In order to obtain the harmonic spectrum, these 16 contributions have to be summed up coherently. At the same time, it is also 
possible to study them separately.

The algorithm of calculation, used in [164], is presented schematically in Figure 6.2 .

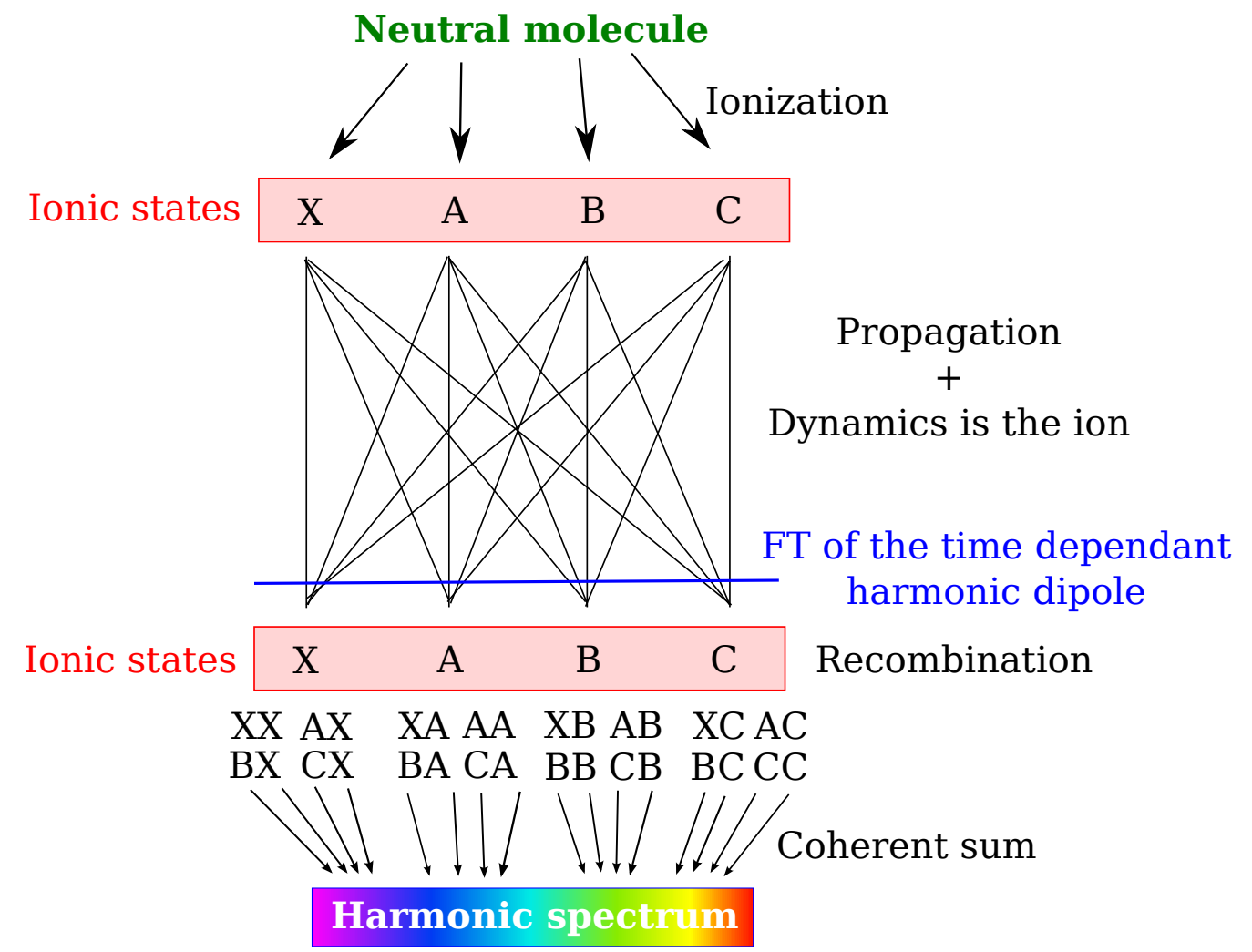

Figure 6.2: Schematic representation of the calculation of the multidimensional high harmonic generation spectra, implemented in [164].

In the calculation in [164] ionization amplitude is computed according to the formula Eq.(3.35), where ionization matrix elements are obtained with $a b$-initio method by [165]. The propagation amplitude is calculated with the formula Eq.(3.37). Dynamics in the ion is simulated with the ab-initio calculation of the TDSE in the basis of four essential ionic states, using the Runge-Kutta algorithm, as described in the Chapter 3.3.3. Finally, the recombination matrix elements for the $\mathrm{CO}_{2}$ were obtained using the ab-initio R-matrix approach in [166].

Let us first examine the experimental two-dimensional HHG spectrum of the 
$\mathrm{CO}_{2}$ molecule. The results, that we are going to consider, were obtained in the experiment [93]. The experimental data of the 2D HHG spectrum of the $\mathrm{CO}_{2}$ aligned at $0^{\circ}$ and $90^{\circ}$ are shown in Figure 6.3. The parameters of the two-color field were: the intensity of the fundamental beam $I \approx 1.3 \cdot 10^{14}$ $\mathrm{W} / \mathrm{cm}^{2}$ with the wavelength $\lambda=800 \mathrm{~nm}$, the intensity conversion ratio for the perpendicularly polarized second harmonic is about $2 \%$. Together with molecules, HHG from the Kr atom was used as a reference, because Kr has similar ionization potential $\left(I_{p}=14.0 \mathrm{eV}\right)$ to the $\mathrm{CO}_{2}$ molecule $\left(I_{p}=13.8 \mathrm{eV}\right)$ and, consequently, close energy of the cut-off harmonic in case of only HOMO contributing to the $\mathrm{CO}_{2} \mathrm{HHG}$ spectrum.

The analysis of the experimental conditions (temperature of the $\mathrm{CO}_{2}$ in the gas chamber) showed, that the range of alignment angles for the molecular ensemble nominally aligned at $0^{\circ}$ has a very wide distribution, and with the volume element taken into account the alignment distribution peaks around $40-45^{\circ}$. More rigorous analysis of the alignment cannot be performed due to lack of data.

Figure 6.3 shows remarkable difference in the position of optimal two-color delay $\phi$ between the molecular and the atomic spectrum. The slope of the curves, corresponding to the $\mathrm{CO}_{2}$, is bent in the direction of smaller two-color phases. As we know from the analysis of the saddle points and the gating technique in the previous chapters, the shape of the optimal phases curve shows the best conditions for each of the steps during the HHG process. The propagation dynamics is only determined by the field configuration, therefore it is the same for the reference atom and the molecule. Thus, the difference between the position of the optimal delays has to signify one or a few of the following processes: the electron-hole dynamics during ionization in molecule, leading to ionization from more than one orbital, the laser-driven dynamics in the ion, and the recombination properties for different molecular orbitals. 

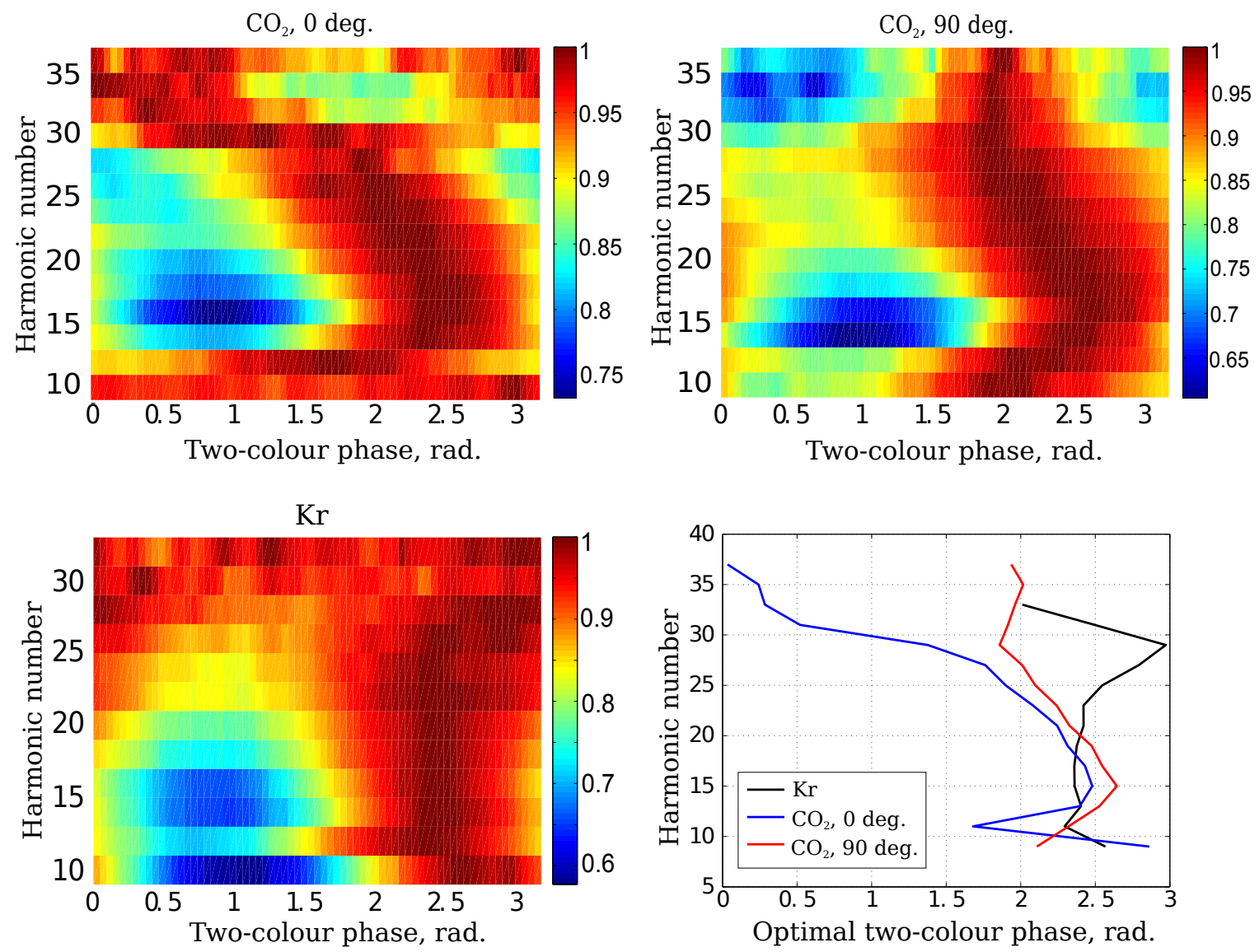

Figure 6.3: Experimental two-dimensional HHG spectra for $\mathrm{CO}_{2}$, aligned at $0^{\circ}$ (top left) and $90^{\circ}$ (top right), for $\mathrm{Kr}$ (bottom left) and positions of optimal two-color phases for all three spectra (bottom right). Experimental results were provided by the group of N. Dudovich, the Weizmann Institute. Parameters of the driving pulse: $I \approx$ $1.3 \cdot 10^{14}, \lambda=800 \mathrm{~nm}$, parameters of the perturbation: $\lambda_{S H}=400$ $\mathrm{nm}, I_{S H}=0.02 I_{0}$.

It is important to note, that ionization dynamics is crucial for the description of HHG in molecules, namely, ionization from different orbitals has to be taken into account and the results from all channels have to be summed up coherently. Similarly, the angle dependence of the ionization rates in molecules is important, when the contributions from several channels are added.

As long as only HOMO orbital is included in the theoretical calculation for $\mathrm{CO}_{2}$, the difference in the ionization amplitudes for atom and molecule lies only 
in the ionization potential of the highest orbitals. Therefore, only XX channel of the process cannot account for the difference between two-dimentional spectra of $\mathrm{CO}_{2}$ and $\mathrm{Kr}$, which is so prominent in the experiment 6.3.

Despite the evidence of the dynamical interference in $\mathrm{CO}_{2}$ molecule during HHG, presented in [127], another experiment [121] seemingly showed no influence of the lower lying orbitals on the harmonic spectrum. High harmonic generation spectrum of the $\mathrm{CO}_{2}$ gas jet, with molecules aligned at $0^{\circ}$, was observed for three wavelengths of the generating linear laser field: $\lambda=1480 \mathrm{~nm}$, $\lambda=1600 \mathrm{~nm}$ and $\lambda=1700 \mathrm{~nm}$. Scan along few intensities was done for each wavelength. The minimum in the spectrum was observed for all wavelengths and intensities at the same energy of the emitted photon, near $60 \mathrm{eV}$. The retrieval procedure, described in [121], obtained the single-molecule contribution to the spectrum in the form of the recombination map of $\mathrm{CO}_{2}$.

Why did this experiment show no evidence of the lower lying orbitals contribution to the HHG spectrum? Weaker coupling between molecular orbitals in the longer wavelength may explain the lack of signature of multielectron dynamics in the linear field experiment. Is it possible, that we need a more sensitive technique to see the multi-electron dynamics during the HHG process?

Let us examine the results more closely. The recombination matrix element of the highest occupied molecular orbital of $\mathrm{CO}_{2}$ has a minimum at $0^{\circ}$ for all recombination energies, because this orbital has a nodal plane at this angle. However, if we look at the alignment distribution of the $\mathrm{CO}_{2}$, we find out that there are no molecules aligned at $0^{\circ}$ due to the $\sin \theta$ volume element. According to the recombination amplitude map, retrieved in [121], the spectrum should show a structural minimum at $60 \mathrm{eV}$ for the molecules aligned at around $45^{\circ}$. Thus, observation of the minimum near $60 \mathrm{eV}$ means that the alignment distribution in the experiment effectively peaked around $45^{\circ}$.

In an attempt to resolve the multi-electron molecular dynamics, the twodimensional measurement of the HHG spectrum in $\mathrm{CO}_{2}$ was done for the wavelength of the fundamental laser field $\lambda=1480 \mathrm{~nm}$, at two intensities of the fundamental field $I_{1} \approx 10^{14} \mathrm{~W} / \mathrm{cm}^{2}$ and $I_{2} \approx 1.4 \cdot 10^{14} \mathrm{~W} / \mathrm{cm}^{2}$.

The intensity conversion ratio for the perturbative second harmonic was about 
$2 \%$. The spectra for the two alignment angles of the molecular ensembles were measured: $0^{\circ}$ and $90^{\circ}$. Published in [164] experimentally measured twodimensional spectra of odd harmonics are presented in Figure 6.4. Figure 6.5 shows the same spectrum, but normalized for each photon energy to its maximum at the optimal two-color delay.
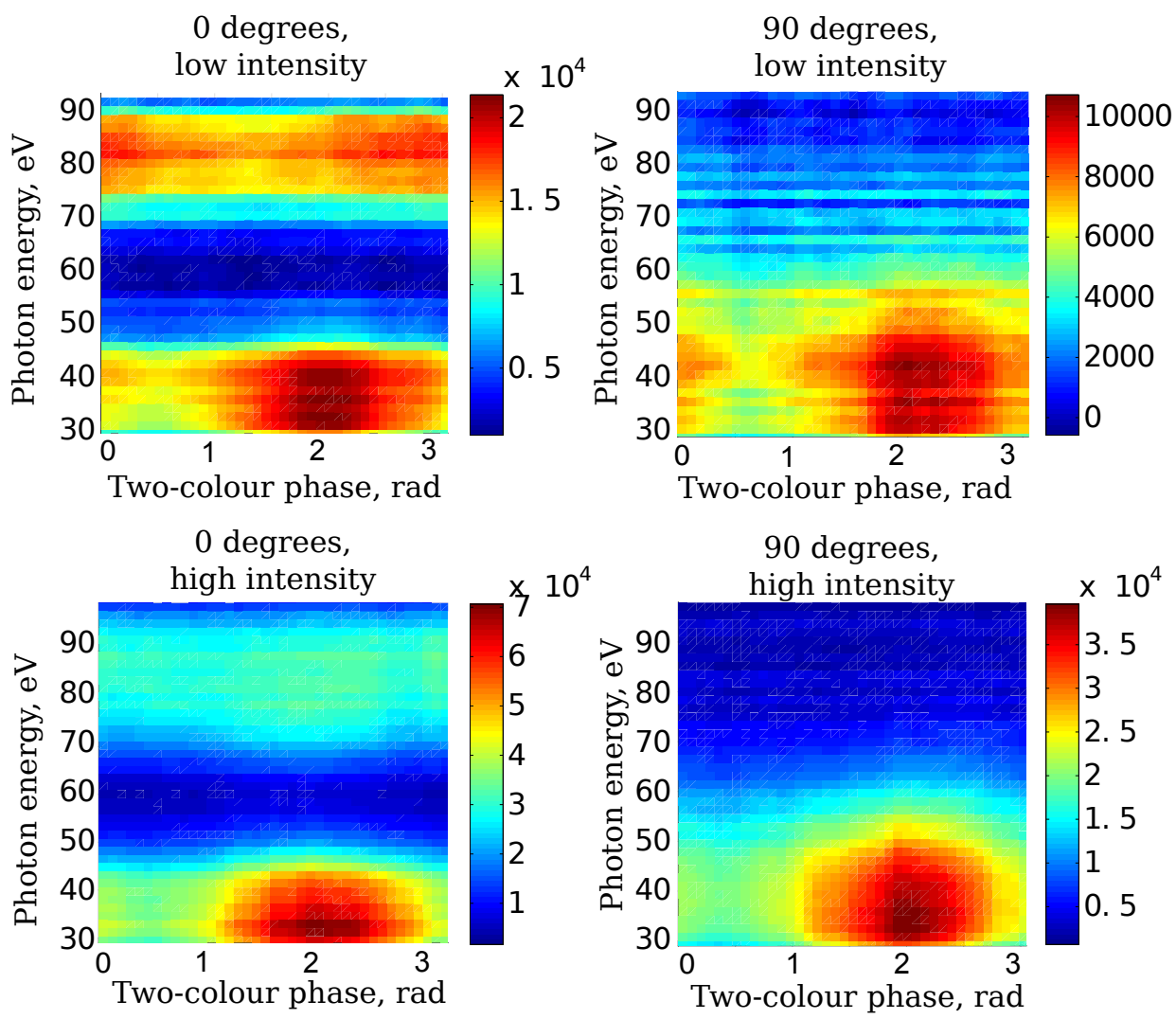

Figure 6.4: Experimental two-dimensional HHG spectra of $\mathrm{CO}_{2}$, aligned at $0^{\circ}$ (top left) and $90^{\circ}$ (top right) for lower intensity $I_{1} \approx 10^{14} \mathrm{~W} / \mathrm{cm}^{2}$, and $0^{\circ}$ (bottom left) and $90^{\circ}$ (bottom right) for higher intensity $I_{2} \approx 1,4 \cdot 10^{14} \mathrm{~W} / \mathrm{cm}^{2}$. Experimental results are courtesy of $\mathrm{N}$. Dudovich and her group at the Weizmann Institute and C. Vozzi and her group at the Polytechnico di Milano.

Given the lack of data regarding the alignment distribution in the experiment, we need to determine the characteristic alignment angle in the experiment. The figure 6.4 of non-normalized 2D harmonic spectrum for the case when the molecular ensemble is parallel to the probing intense field shows prominent minimum for both high and low intensities at $60 \mathrm{eV}$. Given the volume 
element $\sin \theta$, no molecules in the ensemble are perfectly aligned to the polarization of the probing laser field that generates harmonics. Let us examine the recombination matrix element for the $\mathrm{CO}_{2}^{+} \mathrm{X}$ state, obtained using the ab-initio R-matrix method in [166].
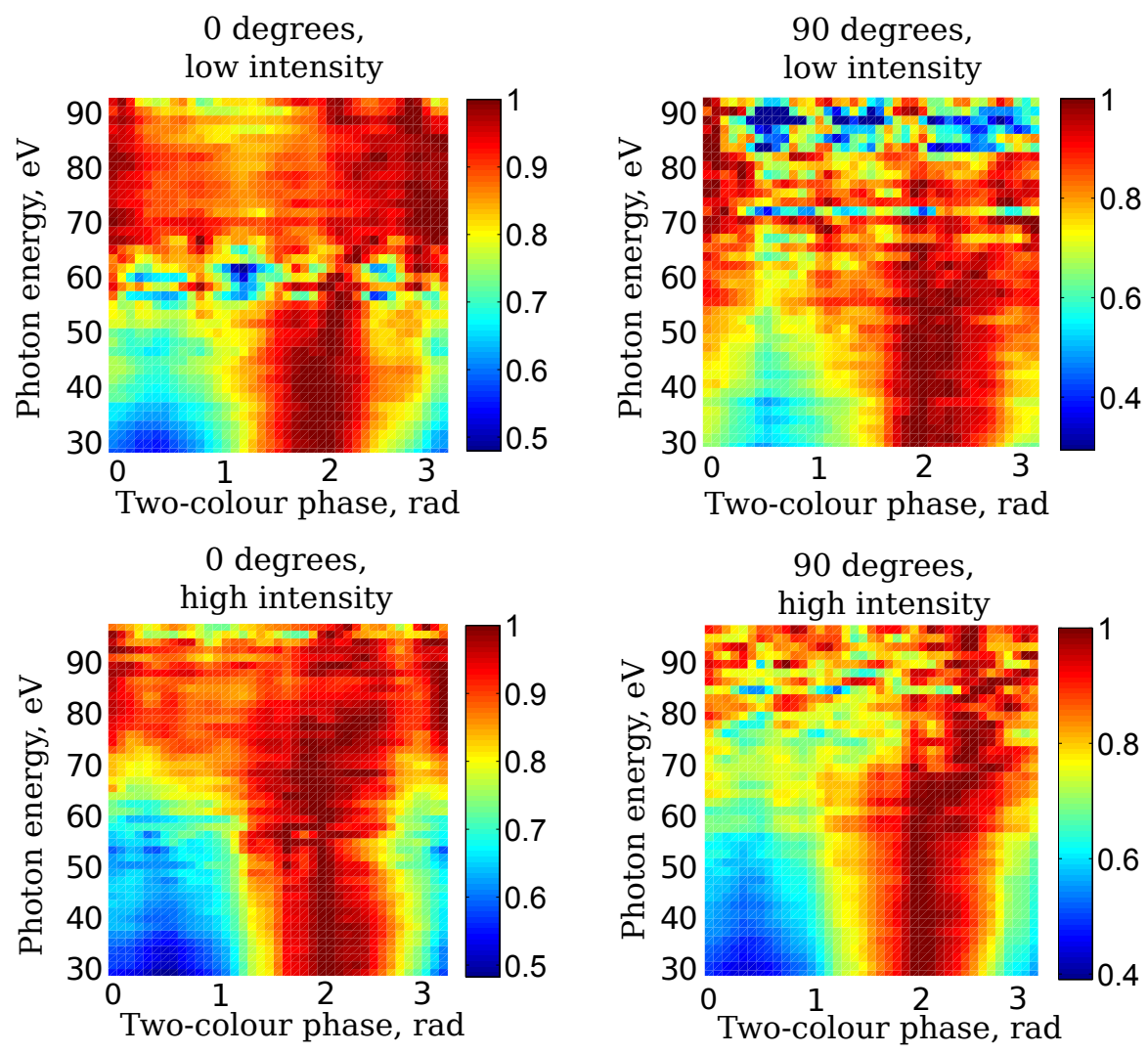

Figure 6.5: Experimental normalized two-dimensional HHG spectra of $\mathrm{CO}_{2}$, aligned at $0^{\circ}$ (top left) and $90^{\circ}$ (top right) for lower intensity $I_{1} \approx$ $10^{14} \mathrm{~W} / \mathrm{cm}^{2}$, and $0^{\circ}$ (bottom left) and $90^{\circ}$ (bottom right) for higher intensity $I_{2} \approx 1,4 \cdot 10^{14} \mathrm{~W} / \mathrm{cm}^{2}$. Experimental results are courtesy of N. Dudovich and her group at the Weizmann Institute and C. Vozzi and her group at the Polytechnico di Milano.

Figure 6.6 shows the amplitude and phase maps of this recombination matrix element for the emitted light with the polarization along the molecular axis (denoted as z-polarization) and with perpendicular polarization (denoted as $\mathrm{x}$-polarization). The minimum of the recombination amplitude at $60 \mathrm{eV}$, that we are looking for, is seen for the $45^{\circ}$ alignment angle for both polarizations of 
emitted light. We conclude, that the effective experimental alignment angles in the ensemble are around $45^{\circ}$.
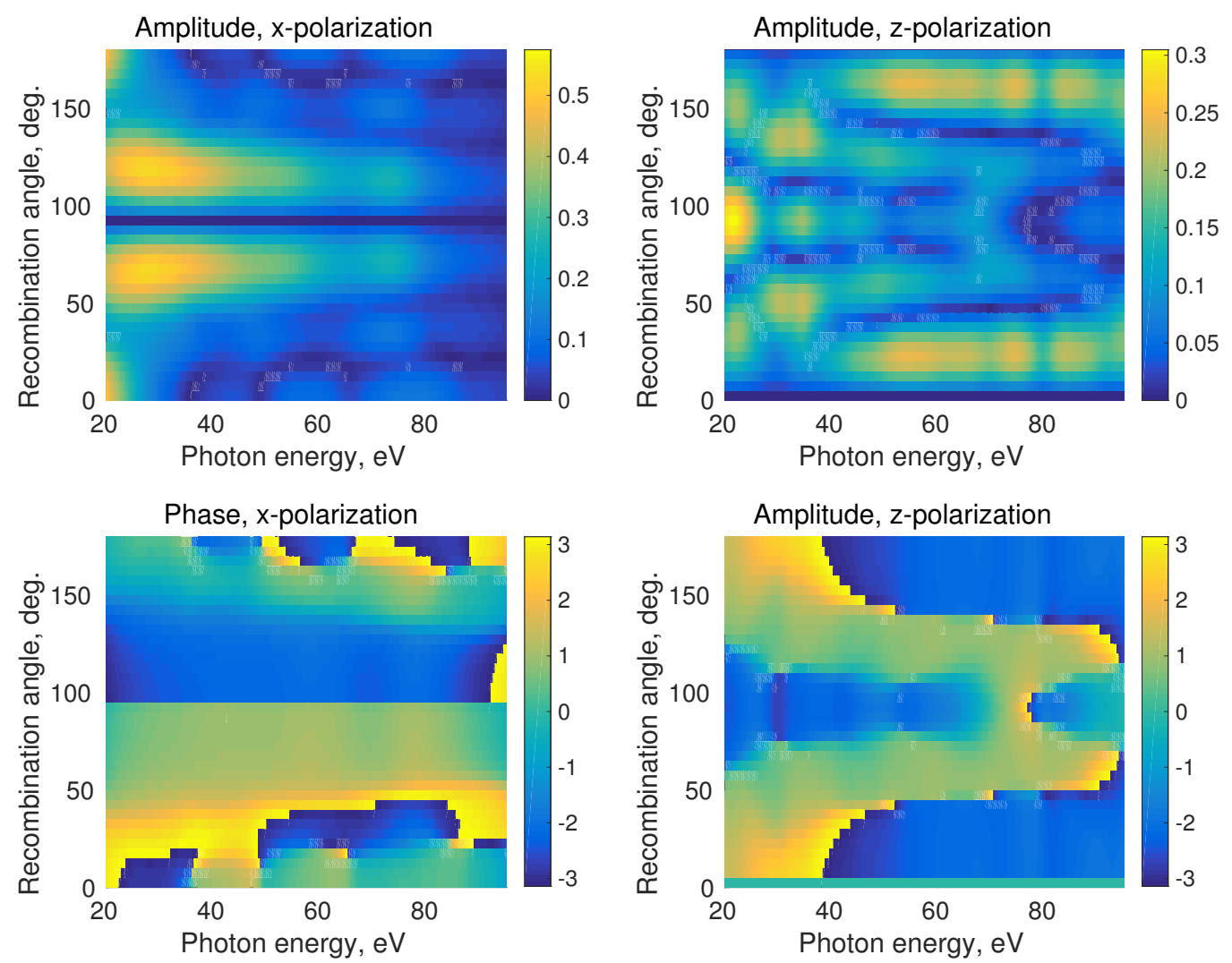

Figure 6.6: Amplitude and phase maps of the recombination matrix element of $\mathrm{CO}_{2} \mathrm{HOMO}$ orbital [166].

The normalized 2D spectrum (figure 6.5) is very informative: we see the shift of the position of the optimal delay at each plot. For the lower intensity the jump in the optimal phase occurs between 60 and $70 \mathrm{eV}$ for the $0^{\circ}$ alignment and between 65 and 75 for the $90^{\circ}$ alignment. For the higher intensity the shift is between 70 and $80 \mathrm{eV}$ for both alignments.

The shift in the position of the optimal two-color phase may be the consequence of one or both of the following factors: the destructive interference between different channels of the harmonic generation and the structural properties of the molecular orbitals. Thorough analysis of these hypothesis was done 
by authors in [164]. Theoretical calculation reproduced experimental results with excellent agreement. Moreover, ionization amplitudes and phases of $\mathrm{CO}_{2}$ were obtained and, thus, dynamics of the hole upon ionization was fully reconstructed.

Important result of the experimental and theoretical investigation in [164] is that the two-dimensional scheme of the HHG brings to the fore the multielectron effects in the longer wavelength, that are not seen in the linear fields, because the scan along the two-color delays changes the contribution of different effects. Indeed, for the same harmonic energy the ion is probed from different recombination angles. All amplitudes (ionization, propagation, population and recombination) depend on the two-color delay to greater or lesser extent. Moreover, this dependence changes with the alignment angle. Thus, the optimization of two-color delay happens at the level of each step of the process with different results for each harmonic number. 


\section{Conclusion}

This Thesis was focused on the theoretical description of the multidimensional high harmonic spectroscopy of atoms and molecules within semi-classical approach.

The introduction into the phenomenon of high harmonic generation was given in the chapter 1 with the description of classical theory ( 3 step model) of HHG, proposed by P. Corkum in [49]. All three steps of the process (ionization, propagation and recombination) were considered in detail separately. History of the study of HHG was also briefly presented in the chapter 1.

Important steps in the development of the high harmonic spectroscopy were described in the chapter 2. Influential theoretical and experimental works on high harmonic generation in atoms and aligned molecules were discussed, as well as pioneering works in the two-color HHG. These works formed the view of high harmonic generation as a very successful tool for studying molecular structure and dynamics.

Semi-classical theory of multidimensional high harmonic generation was presented for the first time in the fullest form in this Thesis in the chapter 3. We calculated high harmonic dipole in the frequency and time domains. Also, very importantly, we included the multielectron effects into the HHG dipole, based on the method by O. Smirnova and M. Ivanov [157].

We developed the theory of multidimensional high harmonic spectroscopy further by studying the particular schemes of multicolor HHG and implementing saddle point analysis in the chapter 4 . Several schemes of multidimensional HHG were studied in detail and following tasks were accomplished: 
- We calculated the amplitude modulation of the semi-classical harmonic dipole moment for the multidimensional HHG.

- We considered three two-color schemes of the generating laser field: strong fundamental field with perturbative perpendicularly polarized second harmonic field (section 4.1.1), strong fundamental field with perturbative parallel polarized second harmonic field (section 4.2) and strong fundamental field with non-perturbative perpendicularly polarized second harmonic field (section 4.1.2).

- Furthermore, we analysed three-color high harmonic generation, when in addition to the fundamental laser wave we add the perturbative perpendicularly polarized field, that consists of second and third harmonic of the fundamental field (section 4.3).

Our analysis described the range of applicability of blind reconstruction employed in (for example, [72]) and in general of strong field approximation for the description of the amplitude modulation of HHG signal in the considered multicolor experiments.

Further, in the chapter 5 we described the implementation of the presented theory to the extraction of the parameters of the electron dynamics during high harmonic generation process from experiment. The first gating experiment with the second harmonic perturbative field, acting as a gate [93], was described together with the reconstruction procedure of the real ionization time and recombination time. However, imaginary ionization time could not be extracted as it affects only the contrast of the modulation, which was not an observable in this experiment.

Two alternative schemes of the HHG experiments were presented in order to gain access to imaginary part of ionization times.

The first option was suggested and implemented in [154]. It is based on the principle of attosecond interferometer. Perturbative second harmonic field with parallel polarization to the fundamental beam is introduced in addition to the fundamental field. The SH field changes the value of the electrical field of the laser during two consecutive half-cycles of the generating field in the opposite directions. As a result, electrons accumulate different phases during 
the tunneling process in each of the half-cycles. This phase is the phase of the temporal interference and is extracted from the experiment.

Imaginary part of ionization time can be reconstructed, using the analysis of the complex phase between interferometer paths. We described how imaginary and real part of the phase can be extracted from the experiment as well as theoretical expressions of this phase within SFA approach. In [154] we showed, that our theoretical calculations of the interference phase and extracted phase from the experiment are in a very good agreement. Based on this fact, reconstruction of the imaginary part of ionization time was done in [154] and it coincides with the SFA value within experimental error bars.

Another technique of the extraction of the ionization parameters from the experiment, that were previously inaccessible, was proposed in [162]. This scheme has the advantage of potential access to all parameters of the electron trajectories. We proposed to use as a perturbation field a composition of second and third harmonics of the fundamental laser wave. Perturbation in this case has perpendicular polarization with respect to the fundamental beam.

Addition of the third harmonic gives another degree of freedom to the experiment. We showed, how control over the phase delay between second and third harmonics affects the regime of HHG: for some multicolor delays the amplitude gate may be considered within classical model, for other delays the modulation of the HHG amplitude can only be described with quantum trajectories. In the latter case the position of the optimal phase delay between fundamental field and SH depends on the imaginary part of ionization time, which essentially means an access to imaginary time via experiment. This property of the particular scheme of multicolor high harmonic spectroscopy allow to implement step-by-step reconstruction of ionization times:

- We need to perform an experiment in three-color HHG with the multicolor delay, which allows to use classical gate for the description of the experiment.

- From this experiment we can extract real ionization time, using procedure described in $[93,162]$ or in this Thesis in section 5.2.2. 
- The second experiment has to be done with the value of the multicolor delay, that gives an access to the imaginary ionization time. In this case, only quantum gate can describe the amplitude modulation.

- We may now reconstruct imaginary part of ionization time from the second experiment, using quantum gate and values of real ionization time, obtained in the step 2.

We presented results of such a procedure in the section 5.2.2. As experimental spectrum we used our simulated HHG spectrum. The results of reconstruction are in a very good agreement with the SFA values of ionization times.

However, it may be problematic in experiments to find out, which of the multicolor phases suit best for the suggested reconstruction. In this case, one may make few experiments with independent values of the multicolor delay. The number of experiments (and different multicolor delays) should be equal to the number of desired reconstructed parameters, because each experimental scan gives an independent equation, discussed in section 5.2.2. In order to obtain all parameters, one would need to solve the system of these equations. In section 5.2.2 we showed an example of reconstruction of real and imaginary ionization times simultaneously via the solution of system of two equations. Just as in the previous reconstruction procedure, we used simulated HHG spectrum as experiment, and the obtained ionization times are in a good agreement with SFA result.

The last chapter 6 of the Thesis gives an outlook on the opportunities presented by two-color HHG spectroscopy to understand the dynamics in $\mathrm{CO}_{2}$ molecule in strong low frequency fields with the aid of the two-color HHG experiments in $800 \mathrm{~nm}$ and $1480 \mathrm{~nm}$ laser light. It was first shown in [127], that multielectron dynamics plays significant role in the generation of HHG spectra of carbon dioxide molecule. Our analysis started with the consideration of the structure of the $\mathrm{CO}_{2}$ molecule.

The first experiment of two-dimensional high harmonic spectroscopy in $\mathrm{CO}_{2}$, that we consider, is HHG in two-color field, when perturbative second harmonic field is perpendicularly polarized with respect to fundamental $800 \mathrm{~nm}$ laser light. The experiment was done by the group of N. Dudovich and the results 
are presented in [93]. Two-dimensional spectrum of $\mathrm{CO}_{2}$ was obtained for two molecular alignments: 0 and 90 degrees. Analysis of the experimental conditions showed, that the real alignment was 45 and 90 degrees.

Further, we consider similar experiment in 2D high harmonic spectroscopy, but with driver with longer wavelength $1480 \mathrm{~nm}$. Experiments, done in [121] in long wavelength, did not seem to show any evidence of the multichannel interference, despite results of [127] and [93]. As in previous case, we showed that the real alignment due to experimental conditions was 45 and 90 degrees. The assessment of the possibilities presented by multidimesional high harmonic spectroscopy, presented in the Thesis, is in line with further investigation by authors in [164]. One-dimensional HHG spectra in long wavelength is not sensitive enough to reveal multi-electron dynamics due to weaker coupling between channels. The position of the optimal phase between two-colors is more sensitive parameter for distinguishing multichannel effects in $\mathrm{CO}_{2}$. 


\section{Bibliography}

[1] M. Drescher, M. Hentschel, R. Kienberger, M. Uiberacker, V. Yakovlev, A. Scrinzi, Th. Westerwalbesloh, U. Kleineberg, U. Heinzmann, and F. Krausz. Time-resolved atomic inner-shell spectroscopy. Nature, 419:803, 2002.

[2] M. Schultze, M. Fieß, N. Karpowicz, J. Gagnon, M. Korbman, M. Hofstetter, S. Neppl, A. L. Cavalieri, Y. Komninos, Th. Mercouris, C. A. Nicolaides, R. Pazourek, S. Nagele, J. Feist, J. Burgdörfer, A. M. Azzeer, R. Ernstorfer, R. Kienberger, U. Kleineberg, E. Goulielmakis, F. Krausz, and V. S. Yakovlev. Delay in photoemission. Science, 328(5986):1658, 2010 .

[3] K. Klünder, J. M. Dahlström, M. Gisselbrecht, T. Fordell, M. Swoboda, D. Guénot, P. Johnsson, J. Caillat, J. Mauritsson, A. Maquet, R. Taïeb, and A. L'Huillier. Probing single-photon ionization on the attosecond time scale. Physical Review Letters, 106:143002, 2011.

[4] A. L. Cavalieri, N. Müller, Th. Uphues, V. S. Yakovlev, A. Baltuška, B. Horvath, B. Schmidt, L. Blümel, R. Holzwarth, S. Hendel, M. Drescher, U. Kleineberg, P. M. Echenique, R. Kienberger, F. Krausz, and U. Heinzmann. Attosecond spectroscopy in condensed matter. $\mathrm{Na}$ ture, 449:1029, 2007.

[5] P. Eckle, A. N. Pfeiffer, C. Cirelli, A. Staudte, R. Dörner, H. G. Muller, M. Büttiker, and U. Keller. Attosecond ionization and tunneling delay time measurements in helium. Science, 322(5907):1525, 2007. 
[6] A. L'Huillier, L. Lompre, G. Mainfray, and C. Manus. Multiply charged ions formed by multiphoton absorption processes in the continuum. Physical Review Letters, 48(26):1814, 1982.

[7] A. L'Huillier, L. Lompre, G. Mainfray, and C. Manus. Multiply charged ions induced by multiphoton absorption in rare gases at $0.53 \mu \mathrm{m}$. Physical Review A, 27(5):2503, 1983.

[8] B. Walker, E. Mevel, B. Yang, P. Breger, J. Chambaret, A. Antonetti, L. Dimauro, and P. Agostini. Double ionization in the perturbative and tunneling regimes. Physical Review A, 48(2):R894, 1993.

[9] B. Walker, B Sheehy, L. Dimauro, P. Agostini, K. Schafer, and K. Kulander. Precision measurement of strong field double ionization of helium. Physical Review Letters, 73(9):1227, 1994.

[10] M. Meckel, D. Comtois, D. Zeidler, A. Staudte, D. Pavičić, H. C. Bandulet, H. Pépin, J. C. Kieffer, R. Dörner, D. M. Villeneuve, and P. B. Corkum. Laser-induced electron tunneling and diffraction. Science, 320(5882):1478, 2008.

[11] T. Zuo, A.D. Bandrauk, and P. B. Corkum. Laser-induced electron diffraction: a new tool for probing ultrafast molecular dynamics. Chemical Physics Letters, 259(3-4):313, 1996.

[12] M. Peters, T. T. Nguyen-Dang, C. Cornaggia, S. Saugout, E. Charron, A. Keller, and O. Atabek. Ultrafast molecular imaging by laser-induced electron diffraction. Physical Review A, 83:051403(R), 2011.

[13] C. I. Blaga, Junliang Xu, Anthony D. DiChiara, Emily Sistrunk, Kaikai Zhang, Pierre Agostini, Terry A. Miller, Louis F. DiMauro, and C. D. Lin. Imaging ultrafast molecular dynamics with laser-induced electron diffraction. Nature, 483:194, 2012.

[14] Y. Huismans, A. Rouzée, A. Gijsbertsen, J. H. Jungmann, A. S. Smolkowska, P. S. W. M. Logman, F. Lépine, C. Cauchy, S. Zamith, T. Marchenko, J. M. Bakker, G. Berden, B. Redlich, A. F. G. van der Meer, H. G. Muller, W. Vermin, K. J. Schafer, M. Spanner, M. Yu. Ivanov, O. Smirnova, D. Bauer, S. V. Popruzhenko, and M. J. J. 
Vrakking. Time-resolved holography with photoelectrons. Science, 331(6013):61, 2011.

[15] P. Agostini, F. Fabre, G. Mainfray, G. Petite, and N. K. Rahman. Freefree transitions following six-photon ionization of xenon atoms. Physical Review Letters, 42:1127, 1979.

[16] F. Lindner, M. G. Schätzel, H. Walther, A. Baltuška, E. Goulielmakis, F. Krausz, D. B. Milošević, D. Bauer, W. Becker, and G. G. Paulus. Attosecond double-slit experiment. Physical Review Letters, 95(040401), 2005 .

[17] A. McPherson, G. Gibson, H. Jara, U. Johann, T. S. Luk, I. McIntyre, K. Boyer, and C. K. Rhodes. Studies of multiphoton production of vacuum-ultraviolet radiation in the rare gases. Journal of the Optical Society of America B, 4(4):595, 2005.

[18] M. Ferray, A. L'Huillier, X. F. Li, L. A. Lomprk, G. Mainfray, and C. Manus. Multiple-harmonic conversion of $1064 \mathrm{~nm}$ radiation in rare gases. Journal of Physics B, 21(4):L31, 1988.

[19] Z. Chang, A. Rundquist, H. Wang, M. Murname, and H. Kapteyn. Generation of coherent soft $\mathrm{X}$ rays at $2.7 \mathrm{~nm}$ using high harmonics. Physical Review Letters, 79(16):2967, 1997.

[20] A. L'Huillier and Ph. Balcou. High-order harmonic generation in rare gases with a 1-ps 1053-nm laser. Physical Review Letters, 70:774, 1993.

[21] J. J. Macklin, J. D. Kmetec, and III C. L. Gordon. High-order harmonic generation using intense femtosecond pulses. Physical Review Letters, 70:766, 1993.

[22] M. D. Perry and G. Mourou. Terawatt to petawatt subpicosecond lasers. Science, 264(5161):917, 1994.

[23] S. G. Preston, A. Sanpera, M. Zepf, W. J. Blyth, C. G. Smith, J. S. Wark, M. H. Key, K. Burnett, M. Nakai, D. Neely, and A. A. Offenberger. High-order harmonics of 248.6-nm KrF laser from helium and neon ions. Physical Review A, 53:R31(R), 1996. 
[24] Ph. Balcou and A. L'Huillier. Phase-matching effects in strong-field harmonic generation. Physical Review A, 47:1447, 1993.

[25] Ph. Balcou, C. Cornaggia, A. S. L. Gomes, L. A. Lompre, and A. L'Huillier. Optimizing high-order harmonic generation in strong fields. Journal of Physics B: Atomic, Molecular and Optical Physics, 25(21):4467, 1992.

[26] Y. Liang, A. Talebpour, C. Y. Chien, S. Augst, and S. L. Chin. Comparison of high harmonic conversion efficiency in atomic and diatomic molecular gases. Journal of Physics B: Atomic, Molecular and Optical Physics, 30(5):1369, 1997.

[27] D. J. Fraser, M. H. R. Hutchinson, J. P. Marangos, Y. L. Shao, J. W. G. Tisch, and M. Castillejo. High harmonic generation in butane and butadiene. Journal of Physics B: Atomic, Molecular and Optical Physics, 28(23):L739, 1995.

[28] Y. Akiyama, K. Midorikawa, Y. Matsunawa, Y. Nagata, M. Obara, H. Tashiro, and K. Toyoda. Generation of high-order harmonics using laser-produced rare-gas-like ions. Physical Review Letters, 69:2176, 1992.

[29] K. Kondo, T. Tamida, Y. Nabekawa, and S. Watanabe. High-order harmonic generation and ionization using ultrashort KrF and Ti:sapphire lasers. Physical Review A, 49:3881, 1994.

[30] N. Sarukura, K. Hata, T. Adachi, R. Nodomi, M. Watanabe, and S. Watanabe. Coherent soft-x-ray generation by the harmonics of an ultrahigh-power KrF laser. Physical Review A, 43:1669, 1991.

[31] C.-G. Wahlström, J. Larsson, A. Persson, T. Starczewski, S. Svanberg, P. Salières, Ph. Balcou, and A. L'Huillier. High-order harmonic generation in rare gases with an intense short-pulse laser. Physical Review A, 48:4709, 1993.

[32] C. Lyngå, A. L'Huillier, and C.-G. Wahlström. High-order harmonic generation in molecular gases. Journal of Physics B: Atomic, Molecular and Optical Physics, 29(14):3293, 1996. 
[33] I. P. Christov, J. Zhou, J. Peatross, A. Rundquist, M. M. Murnane, and H. C. Kapteyn. Nonadiabatic effects in high-harmonic generation with ultrashort pulses. Physical Review Letters, 77:1743, 1996.

[34] K. Kondo, N. Sarukura, K. Sajiki, and S. Watanabe. High-order harmonic generation by ultrashort $\mathrm{KrF}$ and Ti:sapphire lasers. Physical Review A, 47:R2480, 1993.

[35] P. Salières. Coherence properties of the harmonic generation in intense laser field. PhD thesis, Dept. de Recherche sur l'Etat Condense, les Atomes et les Molecules; Paris-6 Univ., 1995.

[36] S. Meyer, H. Eichmann, T. Menzel, S. Nolte, B. Wellegehausen, B. N. Chichkov, and C. Momma. Phase-matched high-order differencefrequency mixing in plasmas. Physical Review Letters, 76:3336, 1996.

[37] P. Salières, A. L'Huillier, and M. Lewenstein. Coherence control of highorder harmonics. Physical Review Letters, 74:3776, 1995.

[38] O. Kfir, P. Grychtol, E. Turgut, R. Knut, D. Zusin, D. Popmintchev, T. Popmintchev, H. Nembach, J. M. Shaw, A. Fleischer, H. Kapteyn, M. Murnane, and O. Cohen. Generation of bright phase-matched circularly-polarized extreme ultraviolet high harmonics. Nature Photonics, 9:99, 2015.

[39] Y. Tamaki, Y. Nagata, M. Obara, and K. Midorikawa. Phase-matched high-order-harmonic generation in a gas-filled hollow fiber. Physical Review A, 59:4041-4044, 1999.

[40] A. Rundquist, C. G. Durfee III, Z. Chang, C. Herne, S. Backus, M. M. Murnane, and H. C. Kapteyn. Phase-matched generation of coherent soft X-rays. Science, 280(5368):1412, 1998.

[41] J. L. Krause, K. J. Schafer, and K. C. Kulander. Calculation of photoemission from atoms subject to intense laser fields. Physical Review A, 45:4998-5010, 1992.

[42] J. H. Eberly, Q. Su, and J. Javanainen. Nonlinear light scattering accompanying multiphoton ionization. Physical Review Letters, 62:881, 1989. 
[43] K. C. Kulander and B. W. Shore. Calculations of multiple-harmonic conversion of 1064-nm radiation in Xe. Physical Review Letters, 62:524, 1989.

[44] K. J. LaGattuta. Laser effects in photoionization. ii. numerical solution of coupled equations for atomic hydrogen. Physical Review A, 41:5110, 1990.

[45] R. M. Potvliege and R. Shakeshaft. Multiphoton processes in an intense laser field: Harmonic generation and total ionization rates for atomic hydrogen. Physical Review A, 40:3061, 1989.

[46] R. A. Sacks and A. Szöke. Electron scattering assisted by an intense electromagnetic field: Exact solution of a simplified model. Physical Review A, 40:5614, 1989.

[47] G. Bandarage., A. Maquet., and J. Cooper. Harmonic generation by a classical hydrogen atom in the presence of an intense radiation field. Physical Review A, 41:1744, 1990.

[48] J. L. Krause, K. J. Schafer, and K. C. Kulander. High-order harmonic generation from atoms and ions in the high intensity regime. Physical Review Letters, 68:3535, 1992.

[49] P. B. Corkum. Plasma perspective on strong field multiphoton ionization. Physical Review Letters, 71:1994, 1993.

[50] K. J. Schafer, B. Yang, L. F. DiMauro, and K. C. Kulander. Above threshold ionization beyond the high harmonic cutoff. Physical Review Letters, 70:1599, 1993.

[51] K. S. Kulander, K. J. Schafer, and J. L. Krause. Super Intense Laser Atom Physics, chapter Dinamics of Short Pulse Excitation, Ionization and Harmonic Conversion. Plenum, New York.

[52] A. L'Huillier, M. Lewenstein, P. Salières, Ph. Balcou, M. Yu. Ivanov, J. Larsson, and C. G. Wahlström. High-order harmonic-generation cutoff. Physical Review A, 48:R3433, 1993. 
[53] M. Lewenstein, Ph. Balcou, M. Yu. Ivanov, A. L'Huillier, and P. B. Corkum. Theory of high-harmonic generation by low-frequency laser fields. Physical Review A, 49:2117, 1994.

[54] K. J. Schafer and K. C. Kulander. Energy analysis of time-dependent wave functions: Application to above-threshold ionization. Physical Review A, 42:5794, 1990.

[55] H. G. Muller and F. C. Kooiman. Bunching and focusing of tunneling wave packets in enhancement of high-order above-threshold ionization. Physical Review Letters, 81:1207, 1998.

[56] B. Sheehy, J. D. D. Martin, L. F. DiMauro, P. Agostini, K. J. Schafer, M. B. Gaarde, and K. C. Kulander. High harmonic generation at long wavelengths. Physical Review Letters, 83:5270, 1999.

[57] M. B. Gaarde, M. Murakami, and R. Kienberger. Spatial separation of large dynamical blueshift and harmonic generation. Physical Review A, 74:053401, 2006.

[58] M. Lezius, V. Blanchet, D. M. Rayner, D. M. Villeneuve, A. Stolow, and M. Yu. Ivanov. Nonadiabatic multielectron dynamics in strong field molecular ionization. Physical Review A, 86:51.

[59] A. N. Markevitch, S. M. Smith, D. A. Romanov, H. B. Schlegel, M. Yu. Ivanov, and R. J. Levis. Nonadiabatic dynamics of polyatomic molecules and ions in strong laser fields. Physical Review A, 68:011402, 2003.

[60] L.V. Keldysh. Ionization in the field of a strong electromagnetic wave. Journal of Experimental and Theoretical Physics, 20(5):1307, 1965.

[61] A.M. Perelomov, V.S. Popov, and M.V. Terent'ev. Ionization of atoms in an alternating electric field. Journal of Experimental and Theoretical Physics, 23(5):924, 1966.

[62] A.M. Perelomov, V.S. Popov, and M.V. Terent'ev. Ionization of atoms in an alternating electric field. ii. Journal of Experimental and Theoretical Physics, 24(1):207, 1967. 
[63] A.M. Perelomov and V.S. Popov. Ionization of atoms in an alternating electric field. iii. Journal of Experimental and Theoretical Physics, 25(2):336, 1967.

[64] N. B. Delone and V. P. Krainov. Atoms in Strong Light Fields. Springer Berlin, 1985.

[65] F. H. M. Faisal. Theory of Multiphoton Processes. Plenum New York, 1987.

[66] H. R. Reiss. Theoretical methods in quantum optics: S-matrix and Keldysh techniques for strong-field processes. Progress in Quantum Electronics, 16:1, 1992.

[67] A. Becker and F. H. M. Faisal. Intense-field many-body S-matrix theory. Journal of Physics B: Atomic, Molecular and Optical Physics, 38(3):R1, 2005

[68] F. Krausz and M. Ivanov. Attosecond physics. Review of Modern Physics, $81: 163,2009$.

[69] F. H. M. Faisal. Multiple absorption of laser photons by atoms. Journal of Physics B: Atomic and Molecular Physics, 6(4):L89, 1973.

[70] H. R. Reiss. Effect of an intense electromagnetic field on a weakly bound system. Physical Review A, 22:1786, 1980.

[71] M. V. Ammosov, N. B. Delone, and V. P. Krainov. Tunnel ionization of complex atoms and of atomic ions in an alternating electromagnetic field. Journal of Experimental and Theoretical Physics, 64(6):1191, 1986.

[72] M. Yu. Ivanov, M. Spanner, and O. Smirnova. Anatomy of strong field ionization. Journal of Modern Optics, 52(2-3):165, 2005.

[73] M. Lein. Molecular imaging using recolliding electrons. Journal of Physics B: Atomic, Molecular and Optical Physics, 40(16):R135, 2007.

[74] S. Baker, J. S. Robinson, C. A. Haworth, C. C. Chirilă, M. Lein, J. W. G. Tisch, and J. P. Marangos. Probing fast nuclear wavepackets in light molecules: monitoring structural rearrangement on an attosecond timescale. Journal of Modern Optics, 54:1011, 2007. 
[75] N. L. Wagner, A. Wüest, I. P. Christov, T. Popmintchev, X. Zhou, M. Murnane, and H. C. Kapteyn. Monitoring molecular dynamics using coherent electrons from high harmonic generation. 103(36):13279, 2006.

[76] R. M. Lock, S. Ramakrishna, X. Zhou, H. C. Kapteyn, M. M. Murnane, and T. Seideman. Extracting continuum electron dynamics from high harmonic emission from molecules. Physical Review Letters, 108:133901, 2012 .

[77] P. M. Paul, E. S. Toma, P. Breger, G. Mullot, F. Augé, Ph. Balcou, H. G. Muller, and P. Agostini. Observation of a train of attosecond pulses from high harmonic generation. Science, 292(5522):1689, 2001.

[78] Z. Q. Yang, D. F. Ye, T. Ding, T. Pfeifer, and L. B. Fu. Attosecond XUV absorption spectroscopy of doubly excited states in helium atoms dressed by a time-delayed femtosecond infrared laser. Physical Review A, 91:013414, 2015.

[79] H. Wang, M. Chini, S. Chen, C.-H. Zhang, F.g He, Y. Cheng, Y. Wu, U. Thumm, and Z. Chang. Attosecond time-resolved autoionization of argon. Physical Review Letters, 105:143002, 2010.

[80] S. Gilbertson, M. Chini, Y. Wu X. Feng, S. Khan, and Z. Chang. Monitoring and controlling the electron dynamics in helium with isolated attosecond pulses. Physical Review Letters, 105:263003, 2010.

[81] G. Gariepy, J. Leach, K. T. Kim, T. J. Hammond, E. Frumker, R. W. Boyd, and P. B. Corkum. Creating high-harmonic beams with controlled orbital angular momentum. Physical Review Letters, 113:153901, 2014.

[82] I. J. Sola, E. Mével, L. Elouga, E. Constant, V. Strelkov, L. Poletto, P. Villoresi, E. Benedetti, J.-P. Caumes, S. Stagira, C. Vozzi, G. Sansone, and M. Nisoli. Controlling attosecond electron dynamics by phasestabilized polarization gating. Nature Physics, 2:219, 2006.

[83] A. Baltuška, Th. Udem, M. Uiberacker, M. Hentschel, E. Goulielmakis, Ch. Gohle, R. Holzwarth, V. S. Yakovlev, A. Scrinzi, T. W. Hänsch, and F. Krausz. Attosecond control of electronic processes by intense light fields. Nature Physics, 2:219, 2006. 
[84] Z. Zeng, R. Li, W. Yu, and Z. Xu. Effect of the carrier-envelope phase of the driving laser field on the high-order harmonic attosecond pulse. Physical Review A, 67:013815, 2003.

[85] G. Sansone, E. Benedetti, J. P. Caumes, S. Stagira, C. Vozzi, M. Nisoli, L. Poletto, P. Villoresi, V. Strelkov, I. Sola, L. B. Elouga, and A. Za. Shaping of attosecond pulses by phase-stabilized polarization gating.

[86] H.-C. Bandulet, D. Comtois, E. Bisson, A. Fleischer, H. Pepin, J.-C. Kieffer, P. B. Corkum, and D. M. Villeneuve. Gating attosecond pulse train generation using multicolor laser fields. Physical Review A, 81:013803, 2010.

[87] Y. Peng and H. Zeng. Pulse shaping to generate an XUV supercontinuum in the high-order harmonic plateau region. Physical Review A, 78:033821, 2008.

[88] E. Goulielmakis, M. Schultze, M. Hofstetter, V. S. Yakovlev, J. Gagnon, M. Uiberacker, A. L. Aquila, E. M. Gullikson, D. T. Attwood, R. Kienberger, F. Krausz, and U. Kleineberg. Single-cycle nonlinear optics. Science, 320(5883):1614, 2008.

[89] G. Sansone, E. Benedetti, F. Calegari, C. Vozzi, L. Avaldi, R. Flammini, L. Poletto, P. Villoresi, C. Altucci, R. Velotta, S. Stagira, S. De Silvestri, and M. Nisoli. Isolated single-cycle attosecond pulses. Science, 314(5798):443, 2006.

[90] A. Zaïr, O. Tcherbakoff, E. Mével, E. Constant, R. Lopez-Martens, J. Mauritsson, P. Johnsson, and A. L'Huillier. Time-resolved measurements of high order harmonics confined by polarization gating. Applied Physics B Lasers and Optics, 78:869, 2004.

[91] C. Vozzi, F. Calegari, F. Ferrari, M. Lucchini, S. De Silvestri, O. Svelto, G. Sansone, S. Stagira, and M. Nisoli. Advances in laser technology for isolated attosecond pulse generation. Laser Physics Letters, 6(4):259, 2009. 
[92] J. Itatani, J. Levesque, D. Zeidler, H. Niikura, H. Pépin, J. C. Kieffer, P. B. Corkum, and D. M. Villeneuve. Tomographic imaging of molecular orbitals. Nature, 432:867, 2004

[93] D. Shafir, Hadas Soifer, B. D. Bruner, M. Dagan, Y. Mairesse, S. Patchkovskii, M. Yu. Ivanov, O. Smirnova, and N. Dudovich. Resolving the time when an electron exits a tunnelling barrier. Nature, 485:343, 2012.

[94] O. Smirnova, S. Patchkovskii, Y. Mairesse, N. Dudovich, and M. Yu. Ivanov. Strong-field control and spectroscopy of attosecond electronhole dynamics in molecules. Proceedings of the National Academy of Sciences, 106(39):16556, 2009.

[95] J. Levesque, D. Zeidler, J. P. Marangos, P. B. Corkum, and D. M. Villeneuve. High harmonic generation and the role of atomic orbital wave functions. Physical Review Letters, 98:183903, 2007.

[96] J. W. Cooper. Photoionization from outer atomic subshells. a model study. Physical Review, 128:681, 1962.

[97] H. J. Wörner, H. Niikura, J. B. Bertrand, P. B. Corkum, and D. M. Villeneuve. Observation of electronic structure minima in high-harmonic generation. Physical Review Letters, 102:103901, 2009.

[98] A. D. Shiner, B. E. Schmidt, C. Trallero-Herrero, P. B. Corkum, J.-C. Kieffer, F. Légaré, and D. M. Villeneuve. Observation of cooper minimum in krypton using high harmonic spectroscopy. Journal of Physics B: Atomic, Molecular and Optical Physics, 45(7):074010, 2012.

[99] H. Stapelfeldt and T. Seideman. Colloquium : Aligning molecules with strong laser pulses. Reviews of Modern Physics, 75:543, 2003.

[100] T. Seideman. Rotational excitation and molecular alignment in intense laser fields. The Journal of Chemical Physics, 103:7887, 1995.

[101] B. Friedrich and D. Herschbach. Alignment and trapping of molecules in intense laser fields. Physical Review Letters, 74:4623, 1995. 
[102] H. J. Loesch and A. Remscheid. Brute force in molecular reaction dynamics: A novel technique for measuring steric effects. The Journal of Chemical Physics, 93:4779, 1990.

[103] N. Hay, R. Velotta, M. Lein, R. de Nalda, E. Heesel, M. Castillejo, and J. P. Marangos. High-order harmonic generation in laser-aligned molecules. Physical Review A, 65:053805, 2002.

[104] J. J. Larsen, H. Sakai., C. P. Safvan, I. Wendt-Larsen, and H. Stapelfeldt. Aligning molecules with intense nonresonant laser fields. The Journal of Chemical Physics, 111:7774, 1999.

[105] M. Gühr. MATLAB code for calculation of rotational revivals and alignment distribution http://web.stanford.edu/ mguehr/research_align.html._Stanford University.

[106] C. Altucci, R. Velotta, J. P. Marangos, E. Heesel, E. Springate, M. Pascolini, L. Poletto, P. Villoresi, C. Vozzi, G. Sansone, M. Anscombe, J.-P. Caumes, S. Stagira, and M. Nisoli. Dependence upon the molecular and atomic ground state of higher-order harmonic generation in the fewoptical-cycle regime. Physical Review A, 71:013409, 2005.

[107] H. Wabnitz, Y. Mairesse, L. J. Frasinski, M. Stankiewicz, W. Boutu, P. Breger, P. Johnsson, H. Merdji, P. Monchicourt, P. Salières, K. Varjú, M. Vitteau, and B. Carré. Generation of attosecond pulses in molecular nitrogen. European Physical Journal, 40:305, 2006.

[108] J. P. Marangos, C. Altucci, R. Velotta, E. Heesel, E. Springate, M. Pascolini, L. Poletto, P. Villoresi, C. Vozzi, G. Sansone, M. Anscombe, J.-P. Caumes, S. Stagira, and M. Nisoli. Molecular orbital dependence of high-order harmonic generation. Journal of Modern Optics, 53(1-2):97, 2006.

[109] C. Altucci, R. Velotta, E. Heesel, E. Springate, J. P. Marangos, C. Vozzi, E. Benedetti, F. Calegari, G. Sansone, S. Stagira, M. Nisoli, and V. Tosa. High-order harmonic generation in alkanes. Physical Review A, 73:043411, 2006. 
[110] M. Plummer and J. F. McCann. Orientation dependence of field ionization of the hydrogen molecular ion. Journal of Physics B: Atomic, Molecular and Optical Physics, 30(11):L401, 1997.

[111] R. Velotta, N. Hay, M. B. Mason, M. Castillejo, and J. P. Marangos. High-order harmonic generation in aligned molecules. Physical Review Letters, 87:183901, 2001.

[112] R. de Nalda, E. Heesel, M. Lein, N. Hay, R. Velotta, E. Springate, M. Castillejo, and J. P. Marangos. Role of orbital symmetry in highorder harmonic generation from aligned molecules. Physical Review A, 69:031804, 2004.

[113] X. Zhou, X. M. Tong, Z. X. Zhao, and C. D. Lin. Alignment dependence of high-order harmonic generation from $\mathrm{N} 2$ and $\mathrm{O} 2$ molecules in intense laser fields. Physical Review A, 72:033412, 2005.

[114] X. Zhou, X. M. Tong, Z. X. Zhao, and C. D. Lin. Role of molecular orbital symmetry on the alignment dependence of high-order harmonic generation with molecules. Physical Review A, 71:061801, 2005.

[115] J. Itatani, D. Zeidler, J. Levesque, Michael Spanner, D. M. Villeneuve, and P. B. Corkum. Controlling high harmonic generation with molecular wave packets. Physical Review Letters, 94:123902, 2005.

[116] R. Torres, N. Kajumba, J. G. Underwood, J. S. Robinson, S. Baker, J. W. G. Tisch, R. de Nalda, W. A. Bryan, R. Velotta, C. Altucci, I. C. E. Turcu, and J. P. Marangos. Probing orbital structure of polyatomic molecules by high-order harmonic generation. Physical Review Letters, 98:203007, 2007.

[117] N. Kajumba, R. Torres, J. G Underwood, J. S. Robinson, S. Baker, J. W. G. Tisch, R. de Nalda, W. A. Bryan, R. Velotta, C. Altucci, I. Procino, I. C. E. Turcu, and J. P. Marangos. Measurement of electronic structure from high harmonic generation in non-adiabatically aligned polyatomic molecules. Physical Review Letters, 98:203007, 2007. 
[118] J. Tate, T. Auguste, H. G. Muller, P. Salières, P. Agostini, and L. F. DiMauro. Scaling of wave-packet dynamics in an intense midinfrared field. Physical Review Letters, 98:013901, 2007.

[119] T. Popmintchev, M.-C. Chen, D. Popmintchev, P. Arpin, S. Brown, S. Ališauskas, G. Andriukaitis, T. Balčiunas, O. D. Mücke, A. Pugzlys, A. Baltuška, B. Shim, S. E. Schrauth, A. Gaeta, C. HernándezGarcía, L. Plaja, Becker A, A. Jaron-Becker, M. M. Murnane, and H. C. Kapteyn. Bright coherent ultrahigh harmonics in the keV X-ray regime from mid-infrared femtosecond lasers. Science, 336(6086):1287, 2012.

[120] R. Torres, T. Siegel, L. Brugnera, I. Procino, Jonathan G. Underwood, C. Altucci, R. Velotta, E. Springate, C. Froud, I. C. E. Turcu, M. Yu. Ivanov, O. Smirnova, and J. P. Marangos. Extension of high harmonic spectroscopy in molecules by a $1300 \mathrm{~nm}$ laser field. Optical Express, 18(3):3174, 2010.

[121] C. Vozzi, M. Negro, F. Calegari, G. Sansone, M. Nisoli, S. De Silvestri, and S. Stagira. Generalized molecular orbital tomography. Nature Physics, 7:822, 2011.

[122] M. Lein, N. Hay, R. Velotta, J. P. Marangos, and P. L. Knight. Role of the intramolecular phase in high-harmonic generation. Physical Review Letters, 88:183903, 2002.

[123] M. Lein, N. Hay, R. Velotta, J. P. Marangos, and P. L. Knight. Interference effects in high-order harmonic generation with molecules. Physical Review A, 66:023805, 2002.

[124] M. Lein, P. P. Corso, J. P. Marangos, and P. L. Knight. Orientation dependence of high-order harmonic generation in molecules. Physical Review A, 67:023819, 2003.

[125] C. Vozzi, F. Calegari, E. Benedetti, J.-P. Caumes, G. Sansone, S. Stagira, M. Nisoli, R. Torres, E. Heesel, N. Kajumba, J. P. Marangos, C. Altucci, and R. Velotta. Controlling two-center interference in molecular high harmonic generation. Physical Review Letters, 95:153902, 2005. 
[126] T. Kanai1, S. Minemoto, and H. Sakai. Quantum interference during high-order harmonic generation from aligned molecules. Nature, 435:470, 2005 .

[127] O. Smirnova, Y. Mairesse, S. Patchkovskii, N. Dudovich, D. Villeneuve, P. Corkum, and M. Yu. Ivanov. High harmonic interferometry of multielectron dynamics in molecules. Nature, 460:972, 2009.

[128] V.-H. Le, A.-T. Le, R.-H. Xie, and C. D. Lin. Theoretical analysis of dynamic chemical imaging with lasers using high-order harmonic generation. Physical Review A, 76:013414, 2007.

[129] V.-H. Le, N.-T. Nguyen, C. Jin, A.-T. Le, and C. D. Lin. Retrieval of interatomic separations of molecules from laser-induced high-order harmonic spectra. Journal of Physics B: Atomic, Molecular and Optical Physics, 41(8):085603, 2008.

[130] H. Niikura, F. Légaré, R. Hasbani, A. D. Bandrauk, M. Yu. Ivanov, D. M. Villeneuve, and P. B. Corkum. Sub-laser-cycle electron pulses for probing molecular dynamics. Nature, 417:917, 2002.

[131] H. Niikura, F. Légaré, R. Hasbani, M. Yu. Ivanov, D. M. Villeneuve, and P. B. Corkum. Probing molecular dynamics with attosecond resolution using correlated wave packet pairs. Nature, 421:826, 2003.

[132] P. P. Corso, R. Daniele, E. Fiordilino, J. P. Marangos, F. Morales, and R. Velotta. Electron and nuclear dynamics of a molecular ion in an intense laser field. Physical Review A, 70:053410, 2004.

[133] N. Hay, M. Castillejo, R. de Nalda, E. Springate, K. J. Mendham, and J. P. Marangos. High-order harmonic generation in cyclic organic molecules. Physical Review A, 61:053810, 2000.

[134] N. Hay, R. de Nalda, T. Halfmann, K. J. Mendham, M. B. Mason, M. Castillejo, and J. P. Marangos. Pulse-length dependence of high-order harmonic generation in dissociating cyclic organic molecules. Physical Review A, 62:041803, 2000.

[135] M. Lein. Attosecond probing of vibrational dynamics with high-harmonic generation. Physical Review Letters, 94:053004, 2005. 
[136] C. C. Chirilă and M. Lein. Influence of nuclear vibration on harmonic generation in molecules. Journal of Physics B: Atomic, Molecular and Optical Physics, 39(13):S437, 2006.

[137] C. C. Chirilă and M. Lein. High-order harmonic generation in vibrating molecules. Journal of Modern Optics, 53(1-2):113, 2006.

[138] C. C. Chirilă and M. Lein. High-order harmonic generation in vibrating two-electron molecules. Chemical Physics, 366:54, 2009.

[139] M. Falge, V. Engel, and M. Lein. Vibrational-state and isotope dependence of high-order harmonic generation in water molecules. Physical Review A, 81:023412, 2010.

[140] S. Baker, J. S. Robinson, C. A. Haworth, H. Teng, R. A. Smith, C. C. Chirilă, M. Lein, J. W. G. Tisch, and J. P. Marangos. Probing proton dynamics in molecules on an attosecond time scale. Science, 312(5772):424, 2006.

[141] W. Li, X. Zhou, R. Lock, S. Patchkovskii, A. Stolow, H. C. Kapteyn, and M. Murnane. Time-resolved dynamics in N2O4 probed using high harmonic generation. Science, 322(5905):1207, 2008.

[142] A.-T. Le, T. Morishita, R. R. Lucchese, and C. D. Lin. Theory of high harmonic generation for probing time-resolved large-amplitude molecular vibrations with ultrashort intense lasers. Physical Review Letters, 109:203004, 2012 .

[143] Y. Mairesse, D. Zeidler, N. Dudovich, M. Spanner, J. Levesque, D. M. Villeneuve, and P. B. Corkum. High-order harmonic transient grating spectroscopy in a molecular jet. Physical Review Letters, 100:143903, 2008.

[144] S.J. Weber, M. Oppermann, and J.P. Marangos. Role of rotational wave packets in strong field experiments. Physical Review Letters, 111:263601, 2013.

[145] Y. Mairesse, S. Haessler, B. Fabrea, J. Higuet, W. Boutu, P. Breger amd E. Constant, D. Descamps, E. Mével, S. Petit, and P. Salières. 
Polarization-resolved pump-probe spectroscopy with high harmonics. New Journal of Physics, 10:025028, 2008.

[146] E. Frumker, C. T. Hebeisen, N. Kajumba, J. B. Bertrand, H. J. Wörner, M. Spanner, D. M. Villeneuve, A. Naumov, and P. B. Corkum. Oriented rotational wave-packet dynamics studies via high harmonic generation. Physical Review Letters, 109:113901, 2012.

[147] S. Patchkovskii, Z. Zhao, T. Brabec, and D. M. Villeneuve. High harmonic generation and molecular orbital tomography in multielectron systems: Beyond the single active electron approximation. Physical Review Letters, 97:123003, 2006.

[148] S. Patchkovskii, Z. Zhao, T. Brabec, and D. M. Villeneuve. High harmonic generation and molecular orbital tomography in multielectron systems. The Journal of Chemical Physics, 126(11):114306, 2007.

[149] Y. Mairesse, J. Higuet, N. Dudovich, D. Shafir, B. Fabre, E. Mével, E. Constant, S. Patchkovskii, Z. Walters, M. Yu. Ivanov, and O. Smirnova. High harmonic spectroscopy of multichannel dynamics in strong-field ionization. Physical Review Letters, 104:213601, 2010.

[150] B. D. Bruner, H. Soifer, D. Shafir, V. Serbinenko, O. Smirnova, and N. Dudovich. Multidimensional high harmonic spectroscopy. Journal of Physics B: Atomic, Molecular and Optical Physics, 48(17):174006, 2015.

[151] N. Dudovich, O. Smirnova, J. Levesque, Y. Mairesse, M. Yu. Ivanov, D. M. Villeneuve, and P. B. Corkum. Measuring and controlling the birth of attosecond XUV pulses. Nature Physics, 2:781, 2006.

[152] H. Niikura, N. Dudovich, D. M. Villeneuve, and P. B. Corkum. Mapping molecular orbital symmetry on high-order harmonic generation spectrum using two-color laser fields. Physical Review Letters, 105:053003, 2010.

[153] J. M. Dahlström, A. L'Huillier, and J. Mauritsson. Quantum mechanical approach to probing the birth of attosecond pulses using a two-colour field. Journal of Physics B: Atomic, Molecular and Optical Physics, 44(9):095602, 2011. 
[154] O. Pedatzur, G. Orenstein, V. Serbinenko, H. Soifer, B. D. Bruner, A. J. Uzan, D. S. Brambila, A. G. Harvey, L. Torlina, F. Morales, O. Smirnova, and N. Dudovich. Attosecond tunnelling interferometry. Nature Physics, 11:815, 2015.

[155] A. Becker and F. H. M. Faisal. Intense-field many-body S-matrix theory. Journal of Physics B: Atomic, Molecular and Optical Physics, 38(3):R1, 2005.

[156] P. Salières, B. Carré, L. Le Déroff, F. Grasbon, G. G. Paulus, H. Walther, R. Kopold, W. Becker, D. B. Milošević, A. Sanpera, and M. Lewenstein. Feynman's path-integral approach for intense-laser-atom interactions. Science, 292(5518):902, 2001.

[157] O. Smirnova and M. Yu. Ivanov. Attosecond and XUV Spectroscopy: Ultrafast Dynamics and Spectroscopy, chapter Multielectron High Harmonic Generation: simple man on a complex plane. Springer, 2014.

[158] D. M. Volkov. Über eine Klasse von Lösungen der Diracschen Gleichung. Zeitschrift für Physik, 94:250, 1935.

[159] M. V. Fedoryuk. Encyclopaedia of Mathematics, chapter Saddle point method. Springer, 2001.

[160] L. Torlina and O. Smirnova. Coulomb time delays in high harmonic generation. New Journal of Physics, 12:023012, 2017.

[161] F. Morales, I. Barth, V. Serbinenko, S. Patchkovskii, and O. Smirnova. Shaping polarization of attosecond pulses via laser control of electron and hole dynamics. Journal of Modern Optics, 59(15):1303, 2012.

[162] V. Serbinenko and O. Smirnova. Multidimensional high harmonic spectroscopy: a semi-classical perspective on measuring multielectron rearrangement upon ionization. Journal of Physics B: Atomic, Molecular and Optical Physics, 46(17):171001, 2013.

[163] W. Boutu, S. Haessler, H. Merdji, P. Breger, G. Waters, M. Stankiewicz, L. J. Frasinski, R. Taieb, J. Caillat, A. Maquet, P. Monchicourt, B. Carre, and P. Salieres. Coherent control of attosecond emission from aligned molecules. Nature Physics, 4:545, 2008. 
[164] B.D. Bruner, Z. Mašìn, M. Negro, F. Morales, D. Brambila, M. Devetta, D. Faccialá, A. Harvey, M. Ivanov, Y. Mairesse, S. Patchkovskii, V. Serbinenko, H. Soifer, S.Stagira, C. Vozzi, N. Dudovich, and O.Smirnova. Multidimentional high harmonic spectroscopy of polyatomic molecules: detecting sub-cycle laser-driven hole dynamics upon ionization in strong mid-IR laser fields. Faraday discussions, 194:369, 2016.

[165] R. Murray, M. Spanner, S. Patchkovskii, and M. Yu. Ivanov. Tunnel ionization of molecules and orbital imaging. Physical Review Letters, 106:173001, 2011.

[166] A. G. Harvey, D. S. Brambila, F. Morales, and O. Smirnova. An R-matrix approach to electron-photon-molecule collisions: photoelectron angular distributions from aligned molecules. Journal of Physics B: Atomic, Molecular and Optical Physics, 47(21):215005, 2014. 